\title{
BEHAVIOURAL SYNTHESIS OF LOW POWER FLOATING POINT CORDIC PROCESSORS
}

\section{SYNTHÈSE COMPORTEMENTALE DE PROCESSEURS CORDIC DE FAIBLE PUISSANCE}

\author{
A Thesis Submitted \\ to the Faculty of the Royal Military College of Canada \\ by \\ Joseph P. Costello, CD, rmc, B.Eng \\ Captain \\ In Partial Fulfillment of the Requirements for the Degree of \\ Master of Engineering in Computer Engineering
}

April 2000

(c) Copyright 2000 by Joseph Costello, Kingston, Ontario. This thesis may be used within the Department of National Defence but copyright for open publication remains the property of the author. 
National Library

of Canada

Acquisitions and Bibliographic Services

395 Wellington Street Ottawa ON K1A ONA Canada
Bibliotheqque nationale du Canada

Acquisitions et services bibliographiques

395. rue Wellington

Ottawa ON KIA ONA

Canada
The author has granted a nonexclusive licence allowing the National Library of Canada to reproduce, loan, distribute or sell copies of this thesis in microform, paper or electronic formats.

The author retains ownership of the copyright in this thesis. Neither the thesis nor substantial extracts from it may be printed or otherwise reproduced without the author's permission.
L'auteur a accordé une licence non exclusive permettant à la Bibliothèque nationale du Canada de reproduire, prêter, distribuer ou vendre des copies de cette thèse sous la forme de microfiche/film, de reproduction sur papier ou sur format électronique.

L'auteur conserve la propriété du droit d'auteur qui protège cette thèse. $\mathrm{Ni}$ la thèse ni des extraits substantiels de celle-ci ne doivent être imprimés ou autrement reproduits sans son autorisation.

\section{Canadä}




\section{ACKNOWLEDGMENTS}

I sincerely and gratefully acknowledge the highly valued assistance, guidance, and encouragement given by Dr. Dhamin Al-Khalili throughout the entire duration of this thesis. His patience and understanding were welcome during those times when nothing seemed to be working as it should.

I would also like to thank those who have helped me to keep things in perspective over a beer or three: Grant, Don, Nick, Dave and Rick.

Finally, I would like to humbly thank my dear wife Michelle who had to endure a great deal to ensure that this work was completed. 


\section{ABSTRACT}

Costello, Joseph P., M.Eng (Computer). Royal Military College of Canada. April 2000. Behavioural Synthesis of Low Power CORDIC Processors.

Supervisor: Dr. Dhamin Al-Khalili.

The COordinate Rotational DIgitial Computer (CORDIC) algorithm has long been used to perform general arithmetic computations. Its strength lies in its ability to perform a wide range of elementary functions, including trigonometric functions, using the same architecture. With the ever increasing need for fast DSP algorithms and implementations, the CORDIC process has recently been under significant investigation. Recent research has led to a wide variety of implementation options, each with its own benefits and drawbacks. With the volume of options available it is becoming increasingly difficult for designers to make the best choices for their application.

This thesis presents the development of a behavioural synthesis tool for the CORDIC process which can produce a total of 576 different versions of the CORDIC processing element suitable for embedded system or system-on-a-chip applications. A suitable algorithm version was chosen, modeled and tested before developing the architectures. The architectures were encoded in the tool and the implementations produced by the tool were characterized. 


\section{RÉSUMÉ}

Costello, Joseph P., M.Eng (Computer). Royal Military College of Canada. Avril 2000. Synthèse comportementale de processeurs CORDIC de faible puissance.

Directeur de thèse: Dr. Dhamin Al-Khalili.

L'algorithme de CORDIC est depuis longtemps utilisé pour l'exécution des calculs généraux d'arithmétiques. Sa force se situe dans sa capacité d'exécuter un éventail de fonctions élémentarires, y compris trigonométriques, en utilisant la même architecture. Avec le besoin toujours croissant pour un signal digital rapide qui traite de l'algorithme et de sa mise en application, le processus de CORDIC a récemment fait l'objet d'une recherche importante. La recherche récente a mené à une grande variété d'options de mise en application, chacune ayant ses propres avantages et inconvénients. Avec le volume d'options disponibles, il devient de plus en plus difficile pour les créateurs de faire les choix les plus appropriés à leur application.

Cette thèse présente le développement d'un outil comportemental de synthèse pour le processus de CORDIC qui peut produire un total de 576 versions différentes d'un CORDIC traitant l'élément approprié au système sur des applications d'une puce. Une version appropriee d'algorithme a été choisie, modelée et testée avant que les architectures n'aient été développées. Les architectures ont été encodées dans l'outil et ses mises en application par l'outil ont été caractérisées. 


\section{TABLE OF CONTENTS}

Page

ABSTRACT $\ldots \ldots \ldots \ldots \ldots \ldots \ldots \ldots \ldots \ldots \ldots \ldots \ldots \ldots \ldots \ldots \ldots \ldots \ldots$ iv

RÉSUMÉ $\ldots \ldots \ldots \ldots \ldots \ldots \ldots \ldots \ldots \ldots \ldots \ldots \ldots \ldots \ldots \ldots \ldots \ldots \ldots$

LIST OF FIGURES $\ldots \ldots \ldots \ldots \ldots \ldots \ldots \ldots \ldots \ldots \ldots \ldots \ldots \ldots \ldots \ldots \ldots$

LIST OF TABLES $\ldots \ldots \ldots \ldots \ldots \ldots \ldots \ldots \ldots \ldots \ldots \ldots \ldots \ldots \ldots \ldots \ldots \ldots \ldots$

LIST OF ABBREVIATIONS $\ldots \ldots \ldots \ldots \ldots \ldots \ldots \ldots \ldots \ldots \ldots \ldots \ldots \ldots \ldots \ldots \ldots$

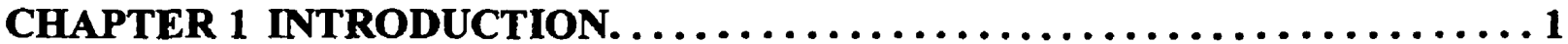

1.1 General Overview. ..........................

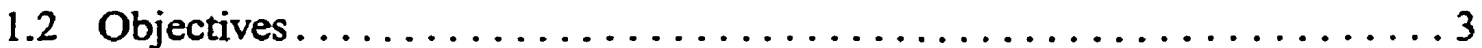

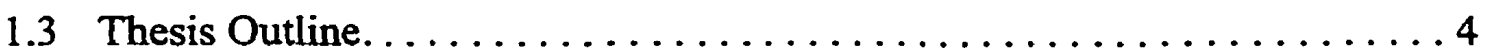

CHAPTER 2 CORDIC BACKGROUND $\ldots \ldots \ldots \ldots \ldots \ldots \ldots \ldots \ldots \ldots \ldots$

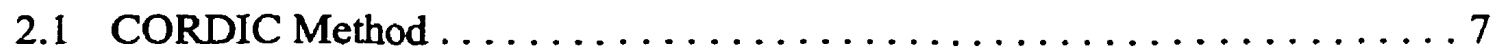

2.2 Unified CORDIC Algorithm .................. 14

2.3 CORDIC Modifications . . . . . . . . . . . . . . . . 16

2.3.1 Hybrid Angle Basis . . . . . . . . . . . . . . . . . . . . . 16

2.3.2 Double Rotation CORDIC . . . . . . . . . . . . . . . . . . . . . . . 18

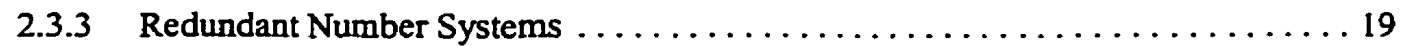

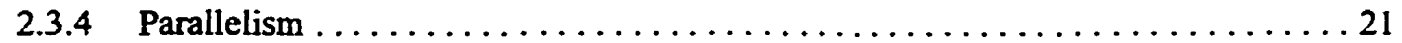

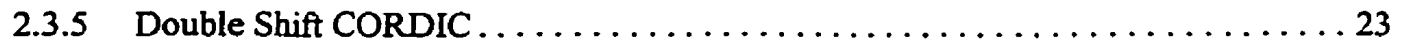

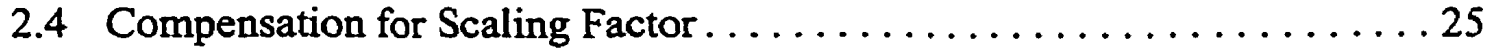

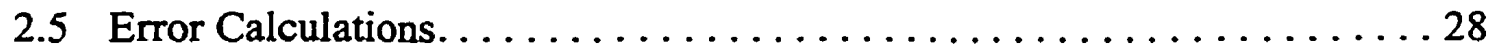

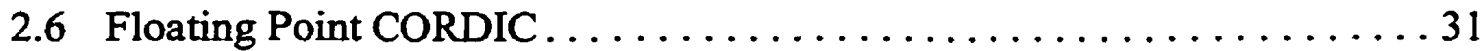

CHAPTER 3 SELECTION OF ALGORITHM . . . . . . . . . . . . . . 36

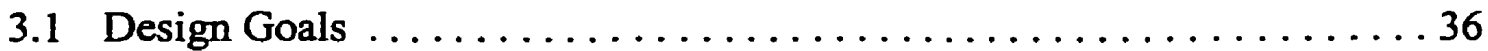

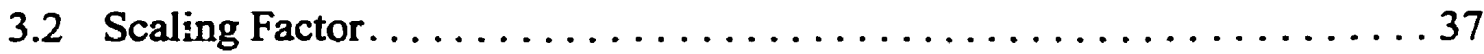

3.3 Number System ....................... 40

3.4 Accuracy............................... 43 
3.5 Latency and Throughput. . . . . . . . . . . . . . . 45

3.6 Selection Decision $\ldots \ldots \ldots \ldots \ldots \ldots \ldots \ldots \ldots \ldots \ldots \ldots \ldots \ldots \ldots \ldots$

CHAPTER 4 ALGORITHM IMPLEMENTATION . . . . . . . . . . . 48

4.1 Input / Output Data. . . . . . . . . . . . . . . . . 48

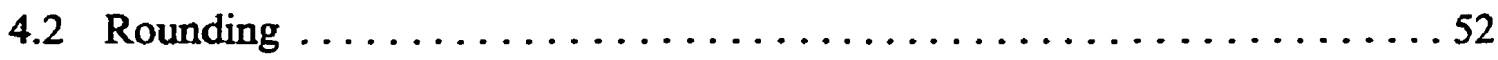

4.3 CORDIC Addition/Subtraction $\ldots \ldots \ldots \ldots \ldots \ldots \ldots \ldots \ldots \ldots \ldots \ldots \ldots \ldots \ldots \ldots$

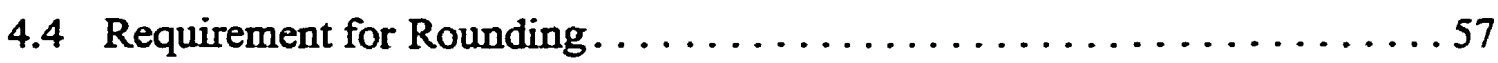

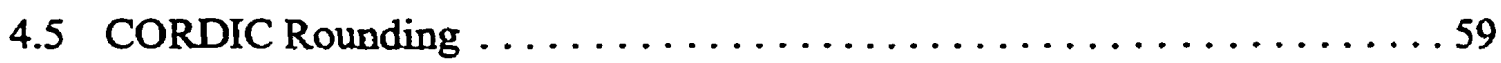

4.5.1 Method One - One Shifted Operand .......................... 60

4.5.2 Method Two - Two Shifted Operands ......................... 62

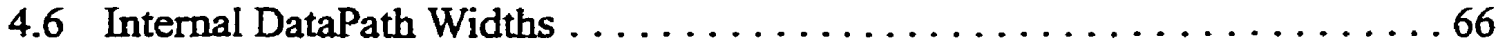

4.7 Iteration Parameters . . . . . . . . . . . . . . . . 68

4.8 Accuracy......................... 73

CHAPTER 5 CORDIC PE ARCHITECTURE. . . . . . . . . . . . . . . 87

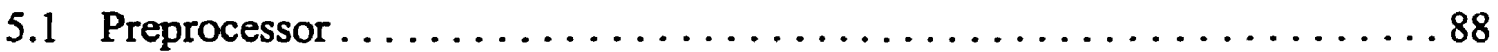

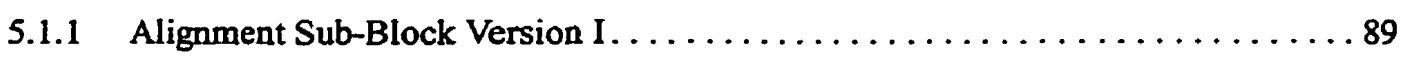

5.1 .2 Alignment Sub-Block Version II . . . . . . . . . . . . . . . . . . 98

5.1 .3 Preproccessor Rotation Sub-Block ....................... 101

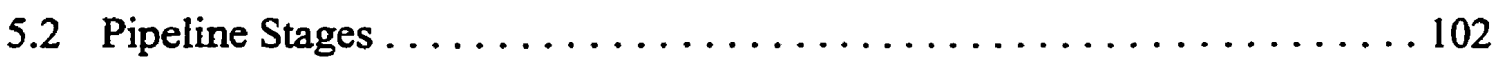

5.2 .1 Double Shift - Full Parallel Block ............................. 103

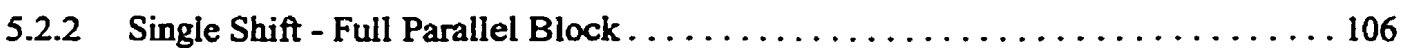

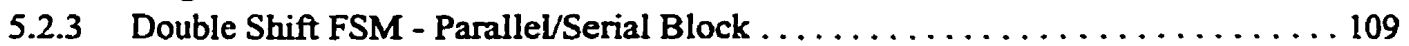

5.2.4 Single Shift FSM - Serial/Parallel Block.................... 113

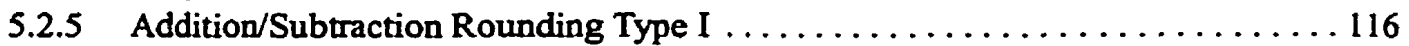

5.2 .6 Addition/Subtraction Rounding Type II. . . . . . . . . . . . . . 117

5.3 Postprocessor . . . . . . . . . . . . . . . . . . . . . . . . . . 119

5.3 .1 Postprocessor Version I . . . . . . . . . . . . . . . . . . . . . 120

5.3 .2 Postprocessor Version II. . . . . . . . . . . . . . . . . . . . . 124

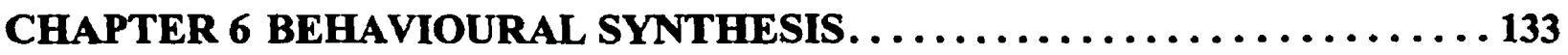

6.1 Behavioural Synthesis Method. . . . . . . . . . . . . 133

6.2 Discussion of Behavioural Synthesis . . . . . . . . . . . 136

6.3 CORDIC Behavioural Synthesis Tool . . . . . . . . . . . 138

6.4 Behavioural Design Flow . . . . . . . . . . . . . . . . . 144

6.5 Tool Operation . . . . . . . . . . . . . . . . . . . 146

6.6 Technical Report. . . . . . . . . . . . . . . . . . . 148

CHAPTER 7 RESULTS . . . . . . . . . . . . . . . . . . . . 149 
7.1 Characterization Environment.................... 149

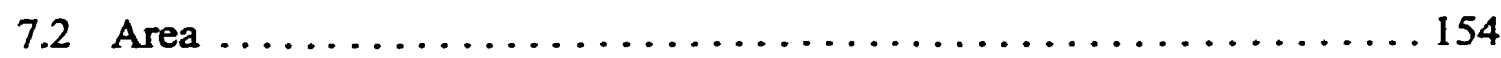

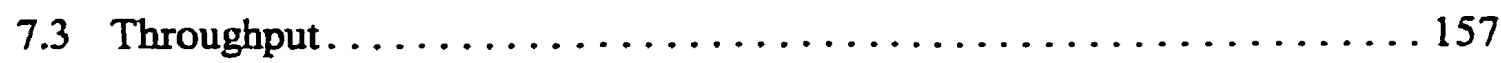

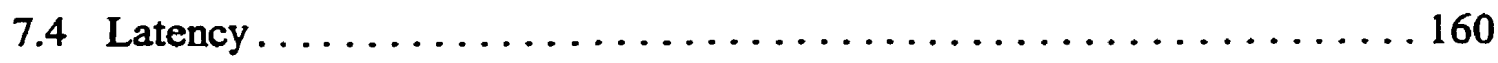

7.5 Energy. . . . . . . . . . . . . . . . . . . . . . 163

7.6 Energy Delay Product. . . . . . . . . . . . . . . . 167

7.7 Energy Area Product . . . . . . . . . . . . . . . . . 170

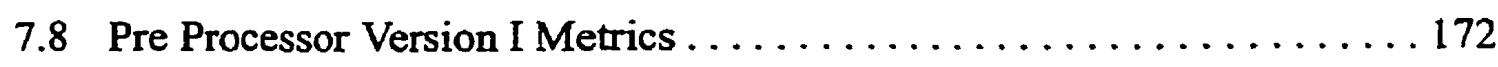

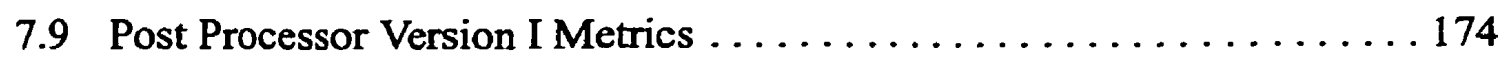

CHAPTER 8 CONCLUSION AND RECOMMENDATIONS . . . . . . . . . 176

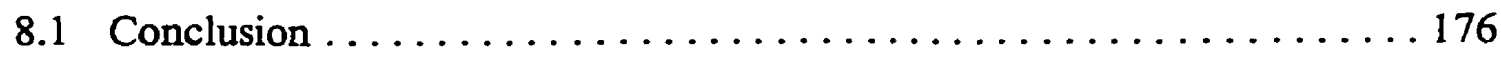

8.2 Recommendations for Future Work . . . . . . . . . . 177

REFERENCES . . . . . . . . . . . . . . . . . . . . . . . . . . . 179

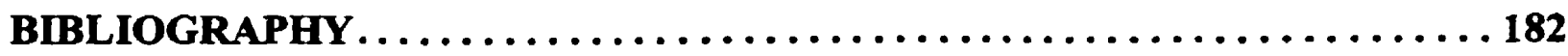




\section{LIST OF FIGURES}

Figure

FIGURE 2.1. Typical CORDIC Computing Step ...............................................13

FIGURE 2.2. Typical Floating Point Mantissa Format .............................................32

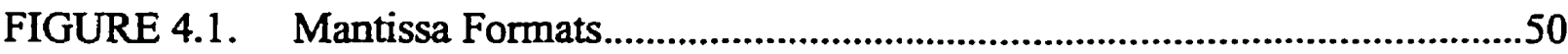

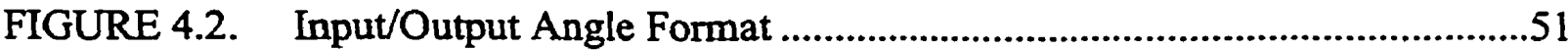

FIGURE 4.3. Generation of Round and Sticky Bits .............................................53

FIGURE 4.4. Resultant with Rounding Bits........................................................54

FIGURE 4.5. Three Term / Two Adder - Addition Options ........................................56

FIGURE 4.6. Pre-addition Operands - Method One...............................................60

FIGURE 4.7. CORDIC Rounding - Method One ....................................................61

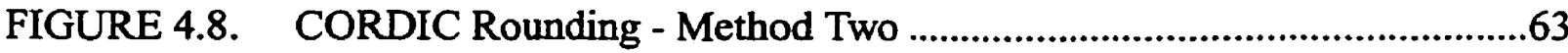

FIGURE 4.9. Post Compression Operands - Method Two ........................................66

FIGURE 4.10. Error Distribution - X Datapath, 12 Bit Mantissa, Vectoring Mode .......77

FIGURE 4.11. Error Distribution - Z Datapath, 12 Bit Mantissa, Vectoring Mode ........77

FIGURE 4.12. Error Distribution - X Datapath, 16 Bit Mantissa, Vectoring Mode .......78

FIGURE 4.13. Error Distribution - Z Datapath, 16 Bit Mantissa, Vectoring Mode ........78

FIGURE 4.14. Error Distribution - X Datapath, 18 Bit Mantissa, Vectoring Mode .......79

FIGURE 4.15. Error Distribution - Z Datapath, 18 Bit Mantissa, Vectoring Mode ........79

FIGURE 4.16. Error Distribution - X Datapath, 24 Bit Mantissa, Vectoring Mode .......80

FIGURE 4.17. Error Distribution - Z Datapath, 24 Bit Mantissa, Vectoring Mode ........80

FIGURE 4.18. Error Distribution - X Datapath, 12 Bit Mantissa, Rotation Mode .........83

FIGURE 4.19. Error Distribution - Y Datapath, 12 Bit Mantissa, Rotation Mode .........83

FIGURE 4.20. Error Distribution - X Datapath, 16 Bit Mantissa, Rotation Mode ........84

FIGURE 4.21. Error Distribution - Y Datapath, 16 Bit Mantissa, Rotation Mode ........84

FIGURE 4.22. Error Distribution - X Datapath, 18 Bit Mantissa, Rotation Mode .........85

FIGURE 4.23. Error Distribution - Y Datapath, 18 Bit Mantissa, Rotation Mode .........85

FIGURE 4.24. Error Distribution - X Datapath, 24 Bit Mantissa, Rotation Mode .........86

FIGURE 4.25. Error Distribution - Y Datapath, 24 Bit Mantissa, Rotation Mode .........86

FIGURE 5.1. Floating Point CORDIC Architecture ................................................87

FIGURE 5.2. Preprocessor Alignment Block - Version I ............................................89

FIGURE 5.3. Evaluation of 'End Around Carry' for $|A-B|$ Computation .....................93 
FIGURE 5.4. Generation of Sum Bits for $|A-B|$ Computation ...................................93

FIGURE 5.5. Data Presentation for Evaluating A - B > K Condition..........................95

FIGURE 5.6. Carry Chain Logic for Evaluating A-B>K Condition ...........................96

FIGURE 5.7. Switch Fabric Latch Control .............................................................97

FIGURE 5.8. Preprocessor Alignment Block - Version II........................................98

FIGURE 5.9. Generation of S5, S6 and S7 of $|A-B|$ Computation ............................99

FIGURE 5.10. Preprocessor Rotation Sub-Block................................................... 101

FIGURE 5.11. Double Shift Pipeline Stage - Fully Parallel Implementation ............... 104

FIGURE 5.12. Z Data Path - Double Shift Pipeline Stage ......................................... 106

FIGURE 5.13. Single Shift Pipeline Stage - Fully Parallel Implementation.................107

FIGURE 5.14. Z Data Path - Single Shift Pipeline Stage .............................................109

FIGURE 5.15. Double Shift FSM Pipeline Stage - Parallel/Serial Implementation ..... 111

FIGURE 5.16. Z Data Path - Double Shift FSM Pipeline Stage .................................112

FIGURE 5.17. Single Shift FSM Pipeline Stage - Parallel/Serial Implementation.......114

FIGURE 5.18. Z Data Path - Single Shift FSM Pipeline Stage ..................................115

FIGURE 5.19. Addition/Subtraction Rounding Type I - One Shifted Operand............117

FIGURE 5.20. Addition/Subtraction Rounding Type II - Two Shifted Operands.........118

FIGURE 5.21. Post Processor without Full Normalization (X Data Path)...................121

FIGURE 5.22. Pseudo Shift Selection of Mantissa Result Bits .................................122

FIGURE 5.23. Selection of Mantissa Result Bits - Version I....................................123

FIGURE 5.24. Selection of Mantissa Result Bits - Version II....................................126

FIGURE 5.25. 16 Bit Leading Zero Counter............................................................128

FIGURE 5.26. Post Processor with Full Normalization (X Data Path) ........................130

FIGURE 5.27. Data Presentation for Exponent Update ...........................................131

FIGURE 6.1. Model Abstraction Level and Synthesis Relationship..........................134

FIGURE 6.2. CORDIC Behavioural Synthesis Tool GUI .......................................142

FIGURE 6.3. Behavioural Design Flow .............................................................145

FIGURE 6.4. CORDIC Behavioural Synthesis Tool Operational Flow ......................146

FIGURE 7.1. Area - No Z Path, Full Parallel .........................................................155

FIGURE 7.2. Area - No Z Path, Serial / Parallel ................................................... 155

FIGURE 7.3. Area - Z Path, Full Parallel ............................................................156

FIGURE 7.4. Area - Z Path, Serial / Parallel ..........................................................156

FIGURE 7.5. Throughput, No Z Path, Full Parallel .............................................. 158

FIGURE 7.6. Throughput, No Z Path, Serial / Parallel ............................................ 159

FIGURE 7.7. Throughput, Z Path, Full Parallel ........................................................159

FIGURE 7.8. Throughput, $Z$ Path, Serial / Parallel ................................................. 160

FIGURE 7.9. Latency - No Z Path, Full Parallel ................................................... 161

FIGURE 7.10. Latency - No Z Path, Serial / Parallel .................................................162

FIGURE 7.11. Latency - Z Path, Full Parallel ........................................................... 162

FIGURE 7.12. Latency - Z Path, Serial / Parallel ...................................................... 163 
FIGURE 7.13. Energy, No Z Path, Full Parallel ......................................................165

FIGURE 7.14. Energy, No Z Path, Serial / Parallel ......................................................165

FIGURE 7.15. Energy, Z Path, Full Parallel .......................................................166

FIGURE 7.16. Energy, Z Path, Serial / Parallel........................................................166

FIGURE 7.17. Energy*Delay Product, No Z Path, Full Parallel..................................168

FIGURE 7.18. Energy*Delay Product, No $Z$ Path, Serial / Parallel ..............................168

FIGURE 7.19. Energy*Delay Product, Z Path, Full Parallel........................................169

FIGURE 7.20. Energy*Delay Product, No Z Path, Serial / Parallel..............................169

FIGURE 7.21. Energy*Area Product, No Z Path, Full Parallel ..................................170

FIGURE 7.22. Energy*Area Product, No Z Path, Serial / Parallel ..............................171

FIGURE 7.23. Energy*Area Product, Z Path, Full Parallel .......................................171

FIGURE 7.24. Energy*Area Product, Z Path, Serial / Parallel ..................................172 


\section{LIST OF TABLES}

Table

Page

TABLE 2.1. Outputs of the CORDIC Algorithm ...................................................15

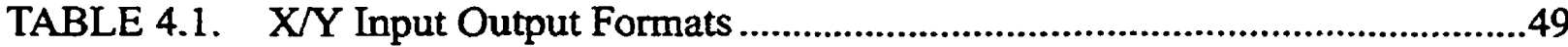

TABLE 4.2. Round to Nearest - Even Scheme .......................................................52

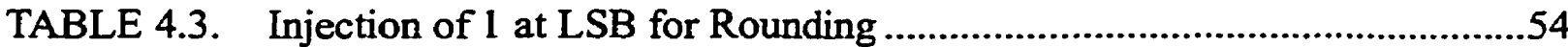

TABLE 4.4. Carry Out Generated by Addition....................................................62

TABLE 4.5. Shift Parameters - 12 bit Accuracy .....................................................71

TABLE 4.6. Shift Parameters - 16 and 18 bit Accuracy ............................................. 72

TABLE 4.7. Shift Parameters - 24 bit Accuracy .......................................................72

TABLE 4.8. Errors in LSB of Result - Vectoring Mode ...........................................76

TABLE 4.9. Errors in LSB of Result - Rotation Mode ...............................................82

TABLE 5.1. Significance of Conditional Carry Outputs..........................................92

TABLE 5.2. Pre-normalization Cases ..................................................................125

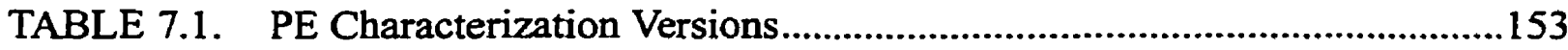

TABLE 7.2. Preprocessor Version I and II Metrics.............................................. 173

TABLE 7.3. Postprocessor Version I and II Metrics ...........................................175 


\section{LIST OF ABBREVIATIONS}

$\begin{array}{ll}\text { ALU } & \text { Arithmetic Logic Unit } \\ \text { ATR } & \text { Arc-Tangent Radix } \\ \text { BSR } & \text { Beyond Shift Range } \\ \text { C/L } & \text { Combinational Logic } \\ \text { CMC } & \text { Canadian Microelectronics Corporation } \\ \text { CORDIC } & \text { COordinate Rotational DIgital Computer } \\ \text { DSP } & \text { Digital Signal Processing } \\ \text { FSM } & \text { Finite State Machine } \\ \text { GUI } & \text { Graphical User Interface } \\ \text { HDL } & \text { Hardware Description Languange } \\ \text { IC } & \text { Integrated Circuit } \\ \text { LSB } & \text { Least Significant Bit } \\ \text { MSB } & \text { Most Significant Bit } \\ \text { PE } & \text { Processing Element } \\ \text { RTL } & \text { Register Transfer Level } \\ \text { SoC } & \text { System on a Chip } \\ \text { TSMC } & \text { Taiwan Semiconductor Manufacturing Company } \\ \text { ulp } & \text { Units of the Last Place }\end{array}$




\section{CHAPTER 1}

\section{INTRODUCTION}

\subsection{General Overview}

The Coordinate Rotational Digital Computer (CORDIC) algorithm has been implemented in a wide variety of applications ranging from general purpose arithmetic logic units (ALUs) to application specific digital signal processing (DSP) filters and matrix equation solvers. The strength of the CORDIC algorithm is that it is able to solve a wide range of elementary functions, including trigonometric functions, natural logarithms and natural exponentials, using a single set of generic equations and a small set of basic operations such as shifting and adding. This strength in generic mathematics can be seen from past implementations of the algorithm in Hewlett Packard calculators and computer arithmetic coprocessors such as the Intel $\times 87$ [1].

The ever increasing speed and data processing requirements of modern DSP applications have led to a shift in focus from software to hardware solutions. The shift is due to the fact that specialized hardware solutions can perform complicated algorithms, on large amounts of data, much faster and so are preferred to software solutions where real time operations are required. 
Concurrent with this trend is the increasing use of the system on a chip (SoC), or embedded systems, approach to hardware design. SoC designs are complex integratedcircuits (ICs) that bring together the major functional elements of a complete end-product into a single chip or chipset [2]. SoC designs feature integrated modules which include memory blocks, controllers, data buses, intellectual property blocks and microprocessors. The exact modules required are design specific and not all designs will have modules of every type. SoC designers need not develop each of these modules from scratch in order to use them but, can instead, use a mixture of predesigned and application specific modules.

In order to achieve design reuse, modules for inclusion in others' designs need to be developed to satisfy a wide range of possible applications. The overall functionality of the module remains unchanged but applications may have different requirements, such as operating speed, data path width, input and output signals etc., which must be accounted for. The use of a module that does not completely meet the design goals results in reduced performance and/or a waste of power and die area. A well designed module, therefore, allows for the specification of various implementation details and is scalable to the application requirements. One method of achieving this is by using an emerging computer aided design method, termed 'behavioural synthesis', in which design implementations reflect specific parameters and constraints applied to a common design description.

Embedded systems and SoC designs exhibit a large increase in design complexity, due to the integration of an increasing number of modules. This leads to some necessary design goals for the modules. The first design goal is small size. Smaller modules are a great benefit as less die space is consumed and, therefore, more is left available for other 
modules. More complex SoC designs can be implemented, with an increased number of modules per die; thus resulting in fewer chips required for a total system design and reduced fabrication costs. The second design goal is low power. Increased design complexity and smaller geometries have led to concerns about power dissipation. Power dissipation is a concern as higher operating temperatures result in a decreased performance and a greater risk of system failure. Power consumption is a concern for modern systems with a large number of components, each of which has increased power requirements due to greater complexity, as there can be a large overall power requirement. This concern is especially important in mobile systems where all power must be supplied by batteries. A decrease in power consumption for mobile systems results in longer operating times and smaller/lighter devices.

\subsection{Objectives}

The aim of this thesis is to develop a CORDIC processing element module, suitable for use in a wide range of embedded systems and SoC applications, using the behavioural synthesis approach.

In order to achieve this aim, a number of objectives must be met. The first is to conduct an in-depth study of the CORDIC algorithm, including recently proposed modifications, in order to determine which version of the algorithm is best suited for a general purpose implementation. After the selection of the algorithm version, it must be developed to meet the needs of the generic CORDIC module. This will entail the determination of the required number of iterations, the iteration parameters, and data path widths, amongst 
other things. The determination of these details will be supported by a data path level algorithm model that will be developed as part of this thesis. The architectures to implement the algorithm in the many forms required for the versatile module, will have to be developed, coded and tested. The output of the architecture model will then have to be verified against the algorithm model to ensure correctness. Once the operation of the various implementations is verified, the next step will be to embed the various implementations into a behavioural synthesis tool which could be used to produce an appropriate version of the CORDIC module based on the designer's requirements. The modules produced by the tool will then have to be verified and characterized in an effort to assist designers in choosing appropriate inputs to the behavioural synthesis.

\subsection{Thesis Outline}

The thesis is broken down into eight chapters. Chapter 2 presents the CORDIC algorithm in its original form and outlines many of the changes and improvements that have been proposed by various researchers. Chapter 3 is a discussion on the relative advantages and disadvantages of the various methods of implementing the CORDIC algorithm in hardware. Chapter 4 describes the preferred algorithm for implementation and provides the additional details required for a successful implementation. Chapter 5 outlines the architecture of the CORDIC processing element and presents the detailed architecture for all of the blocks required to construct it in its various forms. Chapter 6 presents the concept of behavioural synthesis and presents the CORDIC Behavioural Synthesis Tool. Chapter 7 presents the results of the tool and provides characterization data for a subset of the possible architectures in order to provide guidance to the designer in select- 
ing suitable design options. Chapter 8 contains the conclusions and recommendations of this work. 


\section{CHAPTER 2}

\section{CORDIC BACKGROUND}

The basic CORDIC algorithm was first described by Henry Briggs in 1624 in Arithmetica Logarithmica, as stated in [3], although the acronym comes from the modern presentation of a COordinate Rotation DIgital Computer (CORDIC) by Jack Volder in 1959 [4]. Volder developed the algorithm for use in a real-time digital computer for the solution of trigonometric relationships of navigation equations and coordinate transformations. His computer could be controlled to solve either set of the following equations:

$$
\begin{aligned}
& x^{\prime}=K(x \cdot \cos \theta-y \cdot \sin \theta) \\
& y^{\prime}=K(y \cdot \cos \theta+x \cdot \sin \theta)
\end{aligned}
$$

or

$$
\begin{aligned}
& R=K \sqrt{x^{2}+y^{2}} \\
& \phi=\operatorname{atan}(y / x)
\end{aligned}
$$

where $K$ is a constant.

The first set of equations are used for the rotation mode of operation which is now also known as the forward mode, the $\mathrm{z}$ reduction mode or the forward rotation mode. In 
rotation mode the coordinate components of a vector ( $x$ and $y$ ) and a desired angle of rotation $(\theta)$ are provided and the resultant components of the rotated vector are computed.

The second set of equations are used for the vectoring mode of operation which is now also known as the reverse mode, the $y$ reduction mode or the reverse rotation mode. In vectoring mode the coordinate components of a vector are provided and the magnitude and angular arguments of the vector are computed.

\subsection{CORDIC Method}

Volder used the fact that in order to perform a rotation over a desired angle it is possible to apply the entire angle in a single step or, alternatively, as a series of rotations of smaller angles that result in the same overall rotation. The CORDIC algorithm uses these smaller rotations, each of which is termed a micro-rotation, to approximately achieve the desired overall angle of rotation $\theta$.

The CORDIC algorithm is thus an iterative application of micro-rotations using angles $\alpha_{i}$. The iteration equations are shown in equation (2.3) where $\sigma_{i}$ is +1 for counterclockwise rotations and -1 for clockwise rotations. For the rotation mode of operation $x_{i+1}$

$$
\begin{aligned}
& x_{i+1}=x_{i}-\sigma_{i} \cdot y_{i} \cdot \tan \alpha_{i} \\
& y_{i+1}=y_{i}+\sigma_{i} \cdot x_{i} \cdot \tan \alpha_{i} \\
& z_{i+1}=z_{i}-\sigma_{i} \alpha_{i}
\end{aligned}
$$

and $y_{i+1}$ represent the updated coordinates, $z_{i+1}$ represents the remaining angle to be rotated and the initial values are $x_{0}=x_{i n}, y_{0}=y_{i n}$ and $z_{0}=\theta_{i n}$. For the vectoring mode of 
operation $x_{i+1}$ and $y_{i+1}$ represent the updated coordinates, $z_{i+1}$ represents the total angle rotated thus far and the initial values are $x_{0}=x_{i n}, y_{0}=y_{\text {in }}$ and $z_{0}=0$.

These equations are derived from the basic equations that govern the rotation of a vector in cartesian coordinates. These equations are:

$$
\begin{aligned}
& x^{\prime}=x \cdot \cos \alpha-y \cdot \sin \alpha \\
& y^{\prime}=y \cdot \cos \alpha+x \cdot \sin \alpha
\end{aligned}
$$

where $\alpha$ is the angle of rotation.

To arrive at the CORDIC algorithm the equations are rearranged to allow for the cross addition that is the basis of the algorithm. Only the rearrangement for the $x^{\prime}$ equation is shown, but the $y^{\prime}$ is analogous.

$$
\begin{aligned}
x^{\prime} & =x \cdot \cdot \cos \alpha-y \cdot \sin \alpha \\
& =\cos \alpha \cdot\left(x-y \cdot \frac{\sin \alpha}{\cos \alpha}\right) \\
& =\cos \alpha \cdot(x-y \cdot-\tan \alpha)
\end{aligned}
$$

For the CORDIC algorithm the total angle $\theta$ is approximated by a sum of predetermined smaller angles $\alpha_{i}$. The application of the set of smaller angles, in the proper direction, results in an overall rotation close to the desired angle $\theta$. This is shown in equation (2.6) where $\theta$ is the overall effect of the rotations $\alpha_{i}$.

$$
\theta \approx \alpha_{1} \pm \alpha_{2} \pm \alpha_{3} \pm \alpha_{4} \pm \alpha_{5} \pm \ldots \pm \alpha_{n}
$$

As there are only $n$ finite micro-rotation angles it is impossible to represent arbitrary angles $\theta$ without error. The angle approximation error $\delta$ is defined as: 


$$
\delta=\theta-\sum_{i=1}^{n} \sigma_{i} \cdot \alpha_{i}
$$

where $\sigma_{i}$ is the direction of rotation for the micro-angle and is either +1 or -1 . The final value of $\delta$ is determined from the results of the final iteration. Equation (2.8) shows the errors for the rotation and vectoring modes of operation.

$$
\delta= \begin{cases}\operatorname{atan}\left(y_{n} / x_{n}\right) & \text { vectoring mode } \\ z_{n} & \text { rotation mode }\end{cases}
$$

The maximum $\delta$ error value is equal to the smallest angle resolution of the series. This error can be expressed in terms of angle resolution as:

$$
|\delta| \leq \alpha_{n}
$$

To ensure that the rotation can be properly represented, within the degree of accuracy desired, the series must satisfy two conditions. The first condition is that the desired angle of rotation must be able to be represented by the series of micro-angles. This implies that the absolute value of $\theta$ must be less than the sum of all angles plus the allowable error, as shown in equation (2.10), to lie within the zone of convergence.

$$
|\theta| \leq \alpha_{n}+\sum_{j=1}^{n} \alpha_{j}=\theta_{M A X}
$$

The second condition is that the series of angles $\alpha_{i}$ is a decreasing series which converges to zero as shown in equation (2.11). This ensures that if the initial angle $\theta$ lies within the zone of convergence then the solution will converge to zero. 


$$
\alpha_{i}<\alpha_{n}+\sum_{j=i+1}^{n} \alpha_{j}
$$

While it is possible to apply the micro-rotations in either direction, the direction must be chosen according to the mode of operation and the results of the previous iteration. In rotation mode this means that the absolute value of the result $z_{i+1}$ is lesc than the result of the previous iteration $\mathbf{z}_{\mathbf{j}}$. This can be expressed as:

$$
\left|z_{i+1}\right|=|| z_{i}\left|-\alpha_{i}\right|
$$

The choice is made so that the result of the iteration leads to a reduction in the remaining angle to be rotated. The direction in equation (2.3) is chosen such that $\sigma_{i}=\operatorname{sign}\left(z_{i-1}\right)$. A positive angle $z_{i-1}$ will result in a positive (counter clockwise) micro-rotation in iteration $i$. For the vectoring mode of operation the direction of rotation is chosen so that the magnitude of $y$ is reduced. This corresponds to a direction $\sigma_{i}=-\operatorname{sign}\left(y_{i-1}\right)$ being chosen in equation (2.3). A positive value of $y_{i-I}$ will result in a negative (clockwise) micro-rotation $i$.

It is worthy to note that the set of $\left\langle\sigma_{i} ; i=1\right.$ to $\left.\mathrm{n}\right\rangle$ can be regarded as an alternative representation of the angle $\theta$. In some applications it is not necessary to compute the angle update equations for $z$ if the $\sigma$ representation is available, thus saving approximately $1 / 3$ of the computations required per iteration. 
The final form of $x^{\prime}$ as arrived at in equation (2.5) differs mathematically from the equation for $x_{i+1}$ in equation (2.3) by the factor $\cos \alpha_{i}$ which is denoted by $1 / k_{i}$. After $n$ iterations of equation (2.3), the result differs from the desired result by a scale factor $K$,

$$
\begin{aligned}
K & =\prod_{i=1}^{n} k_{i} \\
& =\prod_{i=1}^{n} \frac{1}{\cos \alpha_{i}} \\
& =\prod_{i=1}^{n} \sqrt{1+\left(\tan \alpha_{i}\right)^{2}}
\end{aligned}
$$

The scaling factor $K$ can now be considered as a constant for a given set of microangles $\alpha_{i}$ as described in equation (2.1) and equation (2.2). $K$ is a constant for a given implementation as the set of angles $\alpha_{i}$ is fixed and the direction of rotation by $\alpha_{i}$ has no affect on $k_{i}$ as $\cos \left(\alpha_{i}\right)=\cos \left(-\alpha_{i}\right) . K$ remains constant providing that every angle $\alpha_{i}$ is applied in either the positive or negative direction and is not skipped. This is shown in equation (2.3) where $\sigma_{i}$ is restricted to the values +1 or -1 . As a constant, the scaling factor can be calculated in advance for a given implementation and stored in a lookup table or be 'hard coded' to be later used to recover the desired results.

The set of angles for the micro-rotations is user definable and is called the angle basis. Volder proposed to make the angle basis the set of angles whose arctangent values are a power of 2, termed the Circular Arc Tangent Radix (ATR). 


$$
\begin{array}{ll}
\alpha_{i}=\operatorname{atan}(\pi / 2) & i=1 \\
\alpha_{i}=\operatorname{atan}\left(2^{-(i-2)}\right) & i>1
\end{array}
$$

This is done so that the "rotation" of coordinate components through $\alpha_{i}$ can be achieved by a simple process of shifting and adding. The resultant coordinate components can be obtained by two simultaneous shift-and-add operations thus avoiding expensive multiplication operations that would otherwise be required. This method is the fundamental relationship of the CORDIC computation process.

Equation (2.3) is modified based on this selection of angles so that the equations computed for each micro-rotation are as given below:

$$
\begin{aligned}
& x_{i+1}=x_{i}-\sigma_{i} 2^{-(i-2)} y_{i} \\
& y_{i+1}=y_{i}+\sigma_{i} 2^{-(i-2)} x_{i} \\
& z_{i+1}=z_{i}-\sigma_{i} \alpha_{i}
\end{aligned}
$$

The addition of $x_{i}$ and $y_{i}$ to compute $x_{i+1}$ and $y_{i+1}$ is termed 'cross addition' for obvious reasons.

The application of the circular ATR micro-rotations is governed by the relationships presented in Figure 2.1 where $\theta_{i}$ is the vector's current angle and $\alpha_{i}$ is the microangle of rotation. Each micro-rotation results in a growth in the vector magnitude $R_{i}$ by the factor $k_{i}=\sqrt{1+2^{-2(i-2)}}$ which is the same irrespective of which direction the angle is being rotated. After $n$ micro-rotations the cumulative effect of this growth results in an overall final scaling factor of $K$ as defined in equation (2.13). It is again clear that the scal- 
ing factor depends only on the number of micro-rotations and the rotation angles themselves.

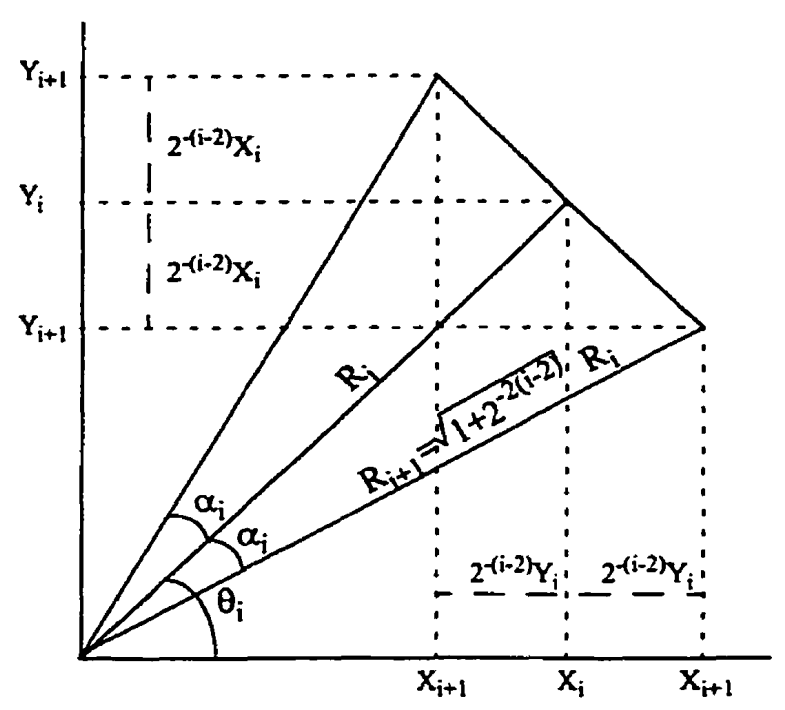

FIGURE 2.1. Typical CORDIC Computing Step

The scaling factor is an unwanted by-product of the micro-rotations and must usually be countered by applying a compensation factor of $1 / K$ to $x^{\prime}$ and $y^{\prime}$ in equation (2.1), or to $R$ in equation (2.2), to obtain the desired final value. This presents a hurdle to the CORDIC process as this is the only time that an apparent multiplication must be performed. One solution is to have a final multiplication stage at the end of the CORDIC iterations. Other methods which have no multiplication have been developed to compensate for the scaling factor. These methods either perform the compensation a little at a time throughout the micro-rotation process or change the scaling factor to be more manageable. This topic is left for a detailed discussion in Section 2.4, Compensation for Scaling Factor. 


\subsection{Unified CORDIC Algorithm}

Since its original publication the CORDIC concept has been expanded and generalized by others. In 1971 Walther [5] developed the unified CORDIC algorithm which included work done to expand the CORDIC algorithm into the linear and hyperbolic coordinate systems. The generalized iteration equations are in the form of

$$
\begin{gathered}
x_{i+1}=x_{i}-m \sigma_{i} 2^{-S(m, i)} y_{i} \\
y_{i+1}=y_{i}+\sigma_{i} 2^{-S(m, i)} x_{i} \\
z_{i+1}=z_{i}-\sigma_{i} \alpha_{m, i}
\end{gathered}
$$

where $m$ equals 1,0 , and -1 for circular, linear and hyperbolic coordinate systems respectively. The direction of rotation still depends on the mode or operation where $\sigma_{i}=\operatorname{sign}\left(z_{i}\right)$ for rotation or $\sigma_{i}=-\operatorname{sign}\left(y_{i}\right)$ for vectoring. $S(m, i)$ refers to the shift sequence for the micro-rotations and, for the circular and linear cases, $S(1, i)=S(0, i)=(0,1,2,3, \ldots)$. This generalized shift sequence differs from that proposed by Volder in the indexing $i$. The generalized sequence starts with index 0 instead of the 1 used in Volder's sequence and Volder's first iteration becomes a non-indexed preshift operation in the generalized sequence. These change the shift amount for a given iteration from $i-2$ to $i$ for the generalized case. The shift sequence for the hyperbolic coordinate system is a modified series with $S(-1, i)=1,2,3,4,4,5, \ldots, 12,13,13,14, \ldots$ where the repeated elements are $\{4,13,40, \ldots, k, 3 k+1, \ldots\}$. This is required to ensure that the angle calculations are convergent. The scaling factor is now 


$$
K_{m, i}=\prod_{i=0}^{n-1} k_{m, i}=\prod_{i=0}^{n-1} \sqrt{1+m \sigma_{i}^{2} 2^{-2 S(m, i)}}
$$

and is only applicable in the circular and hyperbolic coordinate systems. The angle basis for the micro-rotations is formed from the angles calculated by equation (2.18).

$$
\alpha_{m, i}=\frac{1}{\sqrt{m}} \operatorname{atan}\left(\sqrt{m} 2^{-s(m, i)}\right)
$$

The strength of the generalized CORDIC algorithm is that it can calculate more trigonometric functions than the original CORDIC and can also perform simple multiplication and division. The desired results can be obtained by inputting the proper values for $x, y$, and $z$ and choosing the appropriate coordinate system. The outputs of the generalized CORDIC algorithm are summarized in Table 2.1 [6]. To perform multiplication using the

\begin{tabular}{|c|c|c|}
\hline $\begin{array}{c}\text { Coordinate } \\
\text { System }\end{array}$ & $\begin{array}{c}\text { Rotation } \\
z_{n} \rightarrow 0\end{array}$ & $\begin{array}{c}\text { Vectoring } \\
y_{n} \rightarrow 0\end{array}$ \\
\hline $\begin{array}{c}\text { Circular } \\
\mathrm{m}=1\end{array}$ & $\begin{array}{c}x_{n}=k(x \cos (z)-y \sin (z)) \\
y_{n}=k(y \cos (z)+x \sin (z))\end{array}$ & $x_{n}=k \sqrt{x^{2}+y^{2}}$ \\
\hline $\begin{array}{c}\text { Linear } \\
\mathrm{m}=0\end{array}$ & $x_{n}=x$ & $z-\operatorname{atan}\left(\frac{y}{x}\right)$ \\
\hline $\begin{array}{c}y_{n}=y+x \cdot z \\
\text { Hyperbolic } \\
\mathrm{m}=-1\end{array}$ & $x_{n}=k(x \cosh (z)+y \sinh (z))$ & $x_{n}=x$ \\
& $y=k(y \cosh (z)+x \sinh (z))$ & $z_{n}=z+\frac{y}{x}$ \\
\hline
\end{tabular}

\section{TABLE 2.1. Outputs of the CORDIC Algorithm}

general CORDIC, for example, requires the input of the two operands as $x$ and $z$, an input 
of 0 for $y$ and the selection of the linear coordinate system $m=0$. The $y$ result will be $x \cdot z$. This generalized form of the CORDIC equations proved to be so powerful and versatile that it was used by Hewlett-Packard for scientific calculators as well as being the basis for the Intel $x 87$ line of math coprocessors [1].

\subsection{CORDIC Modifications}

Work has continued in the area of the CORDIC algorithm and many modifications to the work by Volder and Walther have been proposed, each aimed at improving the algorithm, mainly with respect to speed, for a particular set of applications. These proposed changes are grouped and presented in the following sections according to the area of the proposed change.

\subsubsection{Hybrid Angle Basis}

It is clear that, due to the nature of the iterative CORDIC equations, one set of computations must be complete before the next may begin. This prevents concurrent computations and represents a bottle-neck in the algorithm. It has been proposed that a mixed set of micro-rotation angles be used that would allow for increased parallelism without the introduction of significant error. This approach is called a Mixed-Hybrid ATR [6].

The angle basis first proposed by Volder, and consisting of angles already discussed, is applied in serial fashion whereby the result from one step determines the direction of rotation in the subsequent step. Another angle basis is called the Linear ATR and it is the set of angles $\alpha_{i}=2^{-i}$. The rotation directions in this case are determined by the 
value of the corresponding $i^{\text {th }}$ bit in the binary angle representation with a 1 and a 0 signifying a positive and a negative rotation respectively. As the direction of one iteration is not dependent on the results of the previous one, it is possible to perform merged CORDIC equations whereby multiple iterations are performed together for faster computations. The problem with the Linear ATR is that the first set of terms in the series introduce a significant amount of error.

The Mixed-Hybrid Circular ATR seeks to eliminate the excessive error and is a combination of the Circular ATR and the Linear ATR consisting of the angles:

$$
\{\overbrace{\operatorname{atan} 2^{-0}, \operatorname{atan} 2^{-1}, \ldots, \operatorname{atan} 2^{-n+1}}^{\text {most significant part }}, \underbrace{2^{-n}, \ldots, 2^{-N+1}}_{\text {least significant }}\}
$$

This Mixed-Hybrid ATR allows the first iterations to be calculated sequentially to avoid unacceptable errors. As the iterations become less significant, and the two ATR terms approach the same value within the required level of accuracy, the remainder of the iterations may be done in some form of parallelism. To facilitate the two styles of computation the angle datapath is split into two parts, one for the most significant $n$ bits and the other for the remaining $N-n$ bits. The algorithm itself is also split into two blocks and is termed the Partitioned-Hybrid CORDIC algorithm. These splits conform to the hybrid-mixed ATR which changes from circular to linear after the first $n$ bits. This approach was presented for sequential implementations of the rotation mode of operation using the circular coordinate system. 


\subsubsection{Double Rotation CORDIC}

A number of researchers have sought to reduce the time required to perform the CORDIC iterations by combining two rotations into one iterative step. This technique is often combined with redundant number representations which also speed up iteration time.

$\mathbf{N}$ Takagi et al. produced a method of double rotation that uses angles of $\operatorname{atan} 2^{-i-1}$ instead of the usual atan $2^{-i}[7],[8]$. This method is based heavily on the use of redundant number representation and is covered in detail in the following section.

Wang and Swartzlander proposed an algorithm where the iterations $i$ and $n-i+I$ were merged to reduce latency [9]. Their algorithm, however, does not allow for the simultaneous computation of the coefficients $\sigma_{i}$ and is, therefore, restricted in use to those cases of the rotation mode where the decomposition of the angle is known beforehand.

Villalba and Lang [10] and Wang and Swartzlander [9] have presented methods in which they proposed to merge two successive iterations. Their methods reduce latency by allowing the concurrent computation of two iterations of the standard CORDIC. This is done by merging the equations for the two iterations and by estimating the second coefficient required. These equations are known as double step or merged iteration equations. The estimation involved in these equations produce increased errors and extra iterations are typically required to obtain the correct result. The modified iteration equations require an additional addition/subtraction calculation but, due to roughly halving the number of iterations required, still result in reduced latency. The CORDIC equations for rotation and 
vectoring modes have been rewritten to allow the coefficient calculations to be performed using a single unified hardware architecture.

\subsubsection{Redundant Number Systems}

It is evident that CORDIC processing involves repeated iterations of the same operations. Any improvement in the iteration speed has a significant effect on the total computation time due to this repetitive nature. One of the most significant delays in the computation process is the carry propagation during the cross addition phase and it is, therefore, the target of optimization efforts. One method of reducing this time is through the use of redundant number systems such as signed digit or carry save representation. The difficulty when introducing redundant number systems is how to quickly evaluate the required sign $\sigma_{i}$ for the next iteration. In Standard CORDIC the sign is equal to the sign bit of $z_{n}$ (for the rotation mode) but in signed digit representation, it is the sign of the most significant non-zero digit of $z_{n}$. This determination requires the examination of an unknown quantity of digits, as many as all of them, which is a slow process.

In order to limit the latency, only a fixed number of digits, say $p$ digits, of $z$ can be examined using a sliding window to determine its sign. The sliding window moves towards the LSB by one bit each iteration as the more significant bits are guaranteed to be zeros. $z$ is determined to have the sign of the most significant non-zero digit examined for the positive and negative case but a third case now exists when the $p$ digits examined are all zero. Although it is possible to not apply $\alpha_{i}$, this leads to the problem of a non-constant 
scaling factor that is not easily dealt with. The problem remains: which way should $\alpha_{i}$ be applied?

N. Takagi et al. proposed a double rotation method that combined the double step CORDIC algorithm with a change in elementary angles from $\operatorname{atan} 2^{-i}$ to $\operatorname{atan} 2^{-i-1}$ [7] [8]. During each iteration the same angle is applied twice vice the use of two successive angles as in the original double step CORDIC. In the case where the sign of $z$ can be determined to be positive or negative, the angle is applied in the corresponding direction in both steps. If, as in the third case, the sign of $z$ can not be determined, the angle is applied in one direction the first step and the opposite direction in the second. In this way the effects of the rotation are nullified but the scaling factor remains constant as the same angles are applied to all computations.

N. Takagi et al. also proposed another method [7] [8], called the correcting rotation method, in which the proper rotation is performed for the first two cases and in the third case the rotation is performed as if $z$ were positive. This leads to increased errors but it is possible to show that a repetition on the iterations $p, 2 p, 3 p, \ldots$, where $p$ represents the number of digits examined to determine the sign, is sufficient to correct the error.

In what has been termed an "ingenious algorithm" J Duprat and J-M Muller [11] presented a new way of performing the iterations with redundant number systems called the Branching CORDIC method. D.S. Phatak has also made contributions in this area with a Double Step Branching Algorithm [12] and with comments and corrections to Duprat and Muller's original paper [13]. The hardware for the Branching CORDIC consists of 
two conventional CORDIC processing cells. When the direction of rotation can be determined, each processing cell performs an identical iteration computation. In the third case where the exact sign of $z$ can not be determined from the examination of a fixed number of bits (usually 3) one processing cell computes as if $z$ were positive and the other as if $z$ were negative. In this way one of the cells will be computing the proper value. This branching condition is allowed to continue until either the required number of iterations have been performed, in which case both computed values are the correct result, or until one of the two computations causes another branching. In the case of a subsequent branching, the value that caused the new branching is taken as the correct result from the original branch as it is the value converging to zero. This conclusion is made since its sign can not be determined meaning that the $p$ digits examined in the sliding window are now zero. This condition indicates that the value of that branch is approaching zero faster than the result of the other branch.

\subsubsection{Parallelism}

Dawid and Meyr proposed a bit level pipelined architecture for use with redundant number representations [14]. In their method, for the rotation mode, they have modified the equation $z_{i+1}=z_{i} \pm \alpha_{i}$ to be $\left|z_{i+1}\right|=|| z_{i}\left|-\alpha_{i}\right|$. The sign of $z_{i}$ is captured while performing the absolute value operation so that it can be used in the calculation of $x_{i+1}$ and $y_{i+1}$. The purpose of this change is to remove the dependence of $z_{i+1}$ on the sign of $z_{i}$.

Once the dependence is removed, and using any redundant number system that allows addition/subtraction and absolute value computations starting from the MSB vice the nor- 
mal LSB, the calculation of $z_{i+l}$ can begin in parallel with the absolute value calculation of $z_{i}$. This parallelism means that the decision for the calculation of $x_{i+1}$ and $y_{i+1}$ is available with only a bit-level delay. Using this technique the number of pipeline stages can be reduced while still maintaining a high throughput. A similar rewriting of the Standard CORDIC equations is done for the vector mode of operation. A draw back of this approach is that the VLSI implementation for the two modes is not the same.

Timmermann et al. proposed two methods for reduced CORDIC latency in conjunction with redundant number representation [15]. One method was by reducing the overhead associated with the $\sigma_{l}=0$ case. In this case other implementations perform a rotation to keep the scaling factor constant. This entails increased iterations and latency. The approach of Timmermann et al. was to adjust the values by the required scaling factor for iterations where $\sigma_{\bar{i}}=0$ instead of performing a rotation. The scaling factor is kept constant and the latency overhead is reduced. Their method exploits the fact that in the higher iterations the effect of the scaling factor becomes less significant. It was found [15] that for iterations $i>(n-3) / 4$ the relation $\sqrt{1+m 2^{-2 i}}=1+m 2^{-2 i-1}$ holds within $n$ bits of precision where $n$ is the total number of iterations and $m$ indicates the coordinate system. Their method allows for $\sigma_{i}=0$ by dealing with it in different ways depending on the iteration $i$. For $0 \leq i \leq(n-3) / 4$ the iterations are done in the usual fashion as expressed in equation (2.16); for $(n-3) / 4 \leq i \leq(n+1) / 2$ the normal CORDIC equations are performed for $\sigma_{i} \neq 0$ but are otherwise replaced with: 


$$
\begin{gathered}
x_{i+1}=x_{i}+m 2^{-2 i-1} x_{i} \\
y_{i+1}=y_{i}+m 2^{-2 i-1} y_{i} \\
z_{i+1}=z_{i}
\end{gathered}
$$

and finally for $i>(n+1) / 2$ the normal CORDIC equations are performed for $\sigma_{i} \neq 0$ but are otherwise replaced with:

$$
\begin{aligned}
& x_{i+1}=x_{i} \\
& y_{i+1}=y_{i} \\
& z_{i+1}=z_{i}
\end{aligned}
$$

The second method proposed by Timmermann et al. was to use $\sigma_{i}$ prediction to reduce the CORDIC latency. Their method performs many iterations in parallel based on a prediction algorithm. At the end of the parallel portion the results are summed and converted back into redundant number representation in order to perform the next set of parallel operations. Some iterations have to be repeated to compensate for the errors generated through the prediction calculations. The set of 'double' iterations is the same as before $\{4,13,40, \ldots, k, 3 k+1\}$ and so the sets of iterations done in parallel are $\{1,4\},\{4,13\},\{13,40\}, \ldots$.

\subsubsection{Double Shift CORDIC}

Work by Deprettere, Dewilde and Udo [16] first presented a modified CORDIC algorithm that allowed for an expanded range of achievable micro-angles. This was achieved by the addition of a third term to the iteration update equations. The third term is 
a shifted value similar to the second term, but with a different shift value. The iteration equations now become:

$$
\begin{gathered}
x_{i+1}=x_{i}-m \sigma_{i}\left(2^{-S(m, i)}+\eta_{i} 2^{-S^{\prime}(m, i)}\right) y_{i} \\
y_{i+1}=y_{i}+\sigma_{i}\left(2^{-S(m, i)}+\eta_{i} 2^{-S^{\prime}(m, i)}\right) x_{i} \\
z_{i+1}=z_{i}-\sigma_{i} \alpha_{m, i}
\end{gathered}
$$

where $S(m, i)$ and $S^{\prime}(m, i)$ are two separate shift sequences and $\eta_{i}$ is allowed values of $-1,0$ and +1 . All other parts of the equations are the same as with the unified CORDIC. In this version of the algorithm the angle basis is redefined such that:

$$
\frac{1}{\sqrt{m}} \tan \left(\sqrt{m} \alpha_{m, i}\right)=2^{-S(m, i)}+\eta_{i} 2^{-S^{\prime}(m, i)}
$$

The addition of the second term in the angle basis allows for a greater flexibility in the choice of angles. The authors deemed this flexibility necessary to take the unified algorithm and implement it efficiently in silicon. The problems cited with the unified equations were, among others:

1. For a given range of angular convergence and accuracy, the computation times for hyperbolic and circular coordinate systems were different;

2. For coordinate system independent execution times the angular zone of convergence and or accuracy had to be impaired; and,

3. Algorithm control would be cumbersome, difficult to design and area consuming. 
The increased degree of freedom allows for the development of iteration parameters, $S(m, i), S^{\prime}(m, i)$ and $\eta_{i}$, which provided coordinate system independent computation time as well as making the scaling factor a single radix 2 shift $K_{m}=2^{-S(m)}$.

This version of the algorithm has been used for such things as fast VLSI filtering[16], a floating-point pipeline CMOS CORDIC processor[17], matrix inversion, direction finding, speech coding and computer graphics[18]. Work has also been done in the area of parameter optimization by Schmidt, Timmermann et al.[19].

\subsection{Compensation for Scaling Factor}

Recall that during a micro-rotation the magnitude $R$ grows by a factor of $k_{i}=\sqrt{1+\sigma_{i}{ }^{2} 2^{-2 S(m, i)}}$ in the case of circular and hyperbolic coordinate systems. This scaling factor must be taken into account and compensated for before arriving at the desired final solution. The scaling factor is a significant hurdle for the CORDIC algorithm to overcome. One of the intentions of the algorithm is to eliminate costly multiplications in favour of shifts and additions. Compensation of the scaling factor is an apparent multiplication operation. With the goal of eliminating the need for even this one multiplication much work has gone into developing alternative methods for the scaling factor compensation.

One way to compensate for the scaling factor is to pre-scale the inputs by $1 / K$ so that the final result will be correct. Another is to ignore it until after the $n^{\text {th }}$ iteration and 
then to perform a multiplication by $1 / K$. These methods impose significant overhead [20] on the CORDIC processing.

A first approach at improvement is to convert $1 / \mathrm{K}$ into a canonical sign-digit representation [21] such that:

$$
\frac{1}{K}=\sum_{p=1}^{P} \kappa_{p} 2^{-i_{p}}
$$

where $\kappa_{p}= \pm 1$ and $i_{p}$ are positive integers so as to reduce the multiplication to $P$ shift and add operations. This method of compensation is performed after the last micro-rotation and uses shifted values of its results. The scaling equations for the $P$ scaling iterations for this solution, with $x^{\prime}(0)=x(n-1)$ and $y^{\prime}(0)=y(n-1)$, are:

$$
\begin{aligned}
& x^{\prime}(i+1)=x^{\prime}(i)+\kappa_{p} 2^{-i_{p}} x(n) \\
& y^{\prime}(i+1)=y^{\prime}(i)+\kappa_{p} 2^{-i_{p}} y(n)
\end{aligned}
$$

Alternatively $1 / K$ may be represented by:

$$
\frac{1}{K}=\prod_{q=1}^{Q}\left(1+\kappa_{q} 2^{-i_{q}}\right)
$$

where again $\kappa_{q}= \pm 1$ and $i_{q}$ are positive integers. In this case the equations for scaling are [22] [23]:

$$
\begin{aligned}
& x^{\prime}(i+1)=\left(1+\kappa 2^{-i}\right) x^{\prime}(i) \\
& y^{\prime}(i+1)=\left(1+\kappa 2^{-i}\right) y^{\prime}(i)
\end{aligned}
$$


which may be performed after $x(n-1)$ has been computed, or in between the standard CORDIC iterations. The exact location of these iterations is not critical due to the commutative properties of multiplication, however, introducing these iterations early in the process may lead to increased errors. The errors are increased since, in the later iterations, the decision about the rotation direction is made on a small number. Errors in the value that the decision is based on can lead to the application of the micro-rotation in the wrong direction [24].

A third solution uses the fact that if certain elementary rotation angles are repeated the resulting scaling factor can be approximated by a power of 2 [23] [25]. The scaling operation in this method consists of several repeated, but standard, micro-rotations and a simple shift operation at the end of the $n^{\text {th }}$ iteration.

The drawback to these solutions is that they increase the latency of the CORDIC algorithm by an amount dependent on the bitwidth and, in some cases, also increase the complexity of the CORDIC iterations [20]. Villalba et al. proposed a method for the parallel compensation of the scaling factor [20], which realizes an iterative compensation for $x_{i}$ and $y_{i}$, performed in parallel with the computation of $x_{i}$ and $y_{i}$, in preparation for the calculation of $x_{i+1}$ and $y_{i+1}$. The basis for the method is to perform the scaling in a distributed fashion by obtaining enough information about each iteration to perform the multiplication in a distributed way. A carry-analysis method is performed on a few bits of $x$ (i) to determine what compensation if required. The hardware used for the compensation computation can be the same as that used for the normal CORDIC operations in serial / paral- 
lel implementations but must be unique in the case of fully pipelined implementations. Their method varies between rotation and vector modes and is described for conventional arithmetic as well as for redundant number systems.

A further method for scaling factor compensation is the steering of the scaling factor through the selection of micro-angles. The scaling factor is dependent on the number, and absolute value, of the basis angles and so a change to the angles has an effect on the scaling factor. In some of the previous methods extra angles have been inserted into the angle basis to achieve this end. The work by Deprettere et al. [16] presented in section 2.3.5 allows for the modification of the angles themselves to achieve the same end without the need for an increased number of micro-rotations.

\subsection{Error Calculations}

In general, errors in the result of a CORDIC process are caused from the finite nature of the implementation. Given an infinite number of bits for the number representations, and an infinite number of iterations, the results could be generated without error but that, of course, is not the case in the real world. Although sometimes described with different categories, errors can be traced to four sources [24] [26]:

1. Input value rounding error - error caused by a finite number of input bits;

2. Angle approximation error - error caused by a finite number of iterations;

3. Rounding error - error caused by a finite iteration width; and,

4. Scaling factor compensation error - error caused by the recovery of the desired result. 
The nature of each of these error sources will be examined below, however, more detailed error equations and derivations are available in the references [5] [24] [26] [27] [28].

The input value rounding error exists for cases where the bitwidth of the datapath, $b$, is less than the bitwidth of the input data, $q$. In cases where the input data has a bitwidth equal to or less than the datapath bitwidth, $q \leq b$, then the error from this source is zero. This error is generated only once when the data enters the CORDIC process and, therefore, occurs irrespective of the number of iterations, $n$, which are subsequently performed. The overall effect of this rounding on the final result is dependent on how sensitive the computation is to changes in the input value. The initial error is propagated to the subsequent iterations where it may be magnified. In general it is not advantageous to let $q$ be greater than b.[26]

In the CORDIC algorithm each rotation angle $\theta$ is approximated by a restricted linear combination of the elementary angles $\left\{\alpha_{m}(i)\right\}$ such that

$$
\theta=\sum_{i=0}^{n-1} \sigma(i) \alpha_{m}(i)+\delta
$$

where $\delta$ is an error due to the angle approximation. The resulting CORDIC computational error due to $\delta$ is called the approximation error. Specific upper bounds are provided in [24] but, in general, to minimize the approximation error the smallest rotation angle $\alpha_{m}(n-1)$ must be made small. This may be achieved by expanding the shift sequence $S(m, n)$ by increasing the number of iterations, $n$. As will be discussed later, the word length $b$ imposes a default upper bound on the number of CORDIC iterations which are required to 
be performed. The smallest rotation angle is, therefore, chosen such that the angle resolution is no smaller than the resolution achievable by the $b$-bit word length of the input data [24].

$$
\alpha_{m}(n-1)=\frac{1}{\sqrt{m}} \operatorname{atan}\left(\sqrt{m} 2^{-S(m, n-1)}\right) \geq 2^{-b}
$$

An optimal iteration number sequence, however, must take into account the effects of rounding error and the CORDIC normalization operations. To reduce the error in the rotation mode of operation, it is desirable to have the maximum rotation angle $\theta_{M A X}$ as large as possible. This can be achieved by expanding the range of $\theta_{M A X}$ as presented in [28]. An increase in the maximum angle on the other hand leads to increased rounding and computational errors due to the increased number of iterations and larger scaling factor. In summary, it is desirable to have $\theta_{M A X} \geq 180^{\circ}$ as a reasonable compromise between the various sources of error [28].

Rounding error results from each iteration of the CORDIC process due to the finite word length of the data path. Worst case error bounds for fixed and floating point arithmetic are shown in [24]. The absolute worst case rounding error for fixed point arithmetic is given in equation (2.30), while the relative worst case rounding error for floating point arithmetic is given in equation (2.31). In the rounding error equations $n$ is the number of iterations, $f(n)$ is the rounding error after $\mathrm{n}$ iterations, $v(n)$ is the final computational result, $\varepsilon$ is the limit of machine accuracy which, for $b$ fractional bits, is $\varepsilon=2^{-b-1}$ and finally 
$|v(n)|_{\text {max }}$ is the largest possible magnitude in the appropriate coordinate system. One way to

$$
\begin{aligned}
|f(n)| \leq n \varepsilon & \\
|f(n)| /|v(n)| \leq n \varepsilon & \text { for } \mathrm{m}=1 \\
|f(n)| /|v(n)|_{\text {max }} \leq n \varepsilon & \text { for } \mathrm{m}=0,-1
\end{aligned}
$$

minimize the effect this error is to expand the internal data path by a number of bits, say $w$ bits, such that equation (2.32) is satisfied. In this way the error will be restricted to the $w$ additional bits and the final results will be accurate to the required $b$-bit width [5].

$$
n \varepsilon=n 2^{-b-w}<2^{-b}
$$

The final source of error is due to the operations performed to compensate for the scaling factor. The amount of the error depends on how the scaling operation is performed. The results are presented in [24] and it is clear that the effects of pre-scaling and in-line scaling with product terms are more significant than that of post-scaling as the rounding error generated from compensation operations affects later iteration computations.

\subsection{Floating Point CORDIC}

The use of fixed point number formats have the disadvantage of not having a dynamic range and accuracy. A given range can only be achieved by adjusting the number of bits in the number representation and so a fixed point CORDIC algorithm would have a set range and accuracy, based on the data path width. Increasing this range quickly leads to bitwidths where the architectures for implementation are unfeasible without very signifi- 
cant increases in area, power and latency. Recall that the number of iterations required is directly proportional to the bitwidth and so larger bitwidths lead to more hardware per iteration but also more iterations. The shortcomings of fixed point number formats can be addressed with a floating point format. Floating point numbers typically consist of three

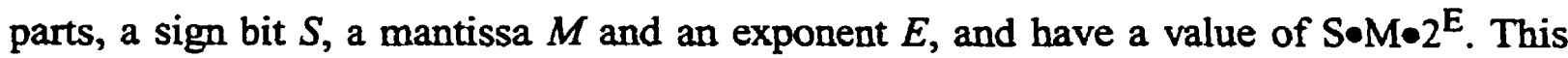
value is based on a placement of the binary bit to the left of the MSB of the mantissa as shown in Figure 2.2. The bitwidth of the mantissa allows for a resolution of $2^{-m+1}$, where $m$ is the bitwidth of the mantissa, which is relative to the exponent value. The exponent bitwidth allows for an increased range by permitting the encoding of a greater range of values. The introduction of the floating point number format, with its dynamic range and accuracy, to the CORDIC process requires that the algorithm be adapted to deal with it. The application of the floating point format to the $x$ and $y$ values and the $z$ values are discussed in the remainder of this section.

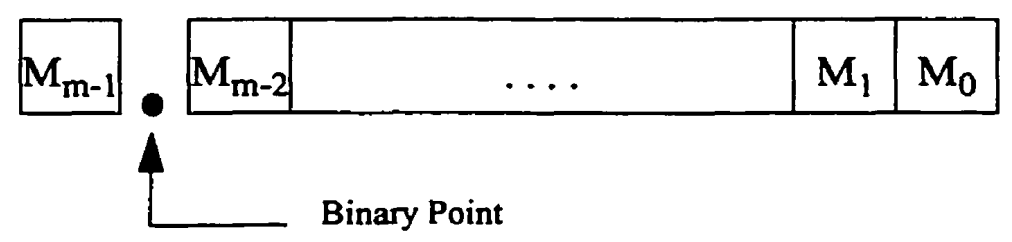

FIGURE 2.2. Typical Floating Point Mantissa Format

A large number of CORDIC implementations to date have utilized floating point number formats [17][18][29][30][31][32][33][34][35]. All of these implementations interface with outside components in floating point format but perform all internal computations in fixed point, also called block floating point, format. This is done to reduce the 
overhead incurred when preparing operands for floating point operations. The addition of two floating point numbers, for example, requires that the operands be aligned so they share a common exponent. This ensures thai the bit positions of the operands have the same value and that the addition can proceed. The result of the addition may then require a normalization step to return the result to the proper floating point format. The alignment and normalization steps are the overhead incurred when using floating point numbers. Block floating point is a way of performing a series of floating point operations without the repetition of the normal alignment and normalization steps. When the data enters the CORDIC processor it is aligned so that the two values ( $x$ and $y$ ) have a common exponent equal to the larger of the two input exponents. This exponent is then stripped from the mantissa and does not rejoin the mantissa until after the final iteration and scaling operations. After it is applied to the mantissa of the result, a normalization step may be performed. The normalization step returns the floating point number to the standard format by shifting the mantissa and adjusting the exponent. In [30] and [31], the exponent that is passed to the normalization block is calculated and applied in different ways depending on what operating mode is being used and what the result is. This modification is done for application specific reasons.

The iteration pipeline operations are performed in fixed point format with steps taken to ensure that what would otherwise cause an overflow condition during the rotation does not occur. As fixed point values, the iteration results can not accommodate a growth in magnitude by a factor of 2 or more as floating point numbers could. Efforts to account for this fact are necessary in order to prevent overflow and the resulting loss of computa- 
tional accuracy. Typically one or more extra bits are added to the left of the MSB to retain relevant data during the iterations.

One of the greatest concerns is with the accuracy of the floating point result. Although it is possible to achieve a result with errors as presented in section 2.5 , the practical implementation is more complicated. Take for example the angle approximation error where the error $\delta$ is based on the shift sequence $S(m, n)$. If the angle in question is small, as is possible in floating point representation, then the corresponding number of iterations required to deal with such small angles would be impracticably large. The question is how to perform floating point CORDIC and maintain results that are accurate to floating point standards?

In some applications, notably matrix computations such as singular value decomposition (SVD) and eigenvalue decomposition, the fine accuracy provided by floating point numbers is not required in the angle path [29]. In processors designed for these types of applications the angle computations can be done in fixed point format and still provide the required level of accuracy.

Methods have been developed by Walther and Johnsson to deal with floating point accuracy [29]. These methods still perform $n$ CORDIC iterations but allow the first iteration to be determined by the most significant non-zero bit in the angle value. Starting with the smallest micro-angle greater than the angle value and continuing for $n$ iterations produces $n$ bits of accuracy in the resulting mantissa. The drawback of this method is that the scaling factor $K$ is no longer constant due to the changes in $\alpha_{i}$ and must either be stored for 
each possible set of iterations or computed in parallel. The first solution consumes a large amount of memory and the second may require a significant investment in hardware.

The implementation by Hekstra and Deprettere in [35] is unique in that it performs a rotation of a floating point angle on the floating point arguments. Presented is a modified algorithm for the circular coordinate system which uses the magnitudes of the operands and the angle to determine an optimal series of micro-rotations. This is done to ensure that the full resolution and accuracy of the floating point notation is preserved. As part of the work, a new method of representing the angle was outlined. In this implementation there is a new pre-rotation stage prior to the alignment stage that results in a restriction on the possible number of optimal rotation series. As before, each series results in a unique scaling factor $K$ but the range of possible series has been reduced to ease the computational overhead. It is shown in the implementation that the additional errors incurred in this process are less than the precision of the inputs irrespective of the operand values and, therefore, result in no loss of accuracy. 


\section{CHAPTER 3}

\section{SELECTION OF ALGORITHM}

It is evident from the number of variations on the basic CORDIC process that some thought is required when choosing a method for a specific application. This chapter presents an examination of the choices available with an aim of discovering the best method for a generic CORDIC processing element (PE). It is clear that choices in many of the areas affect the possible choices in other areas. The final set of method / design decisions must be advantageous when taken as a whole. This may lead to sub-optimal choices in one or more areas in order to gain some advantage in another. In order to properly evaluate these trade-offs and select the proper characteristics for the generic CORDIC PE it is

important to first review the design goals against which the various options can be weighed. The sections following the review of the design goals present discussions on the various design issues.

\subsection{Design Goals}

The following is a summary of the goals which govern the design of a generic CORDIC PE. These goals are derived from both the need for generality in order to satisfy 
a wide range of applications and the requirements of modern systems. The principle goals are:

1. Target Applications - The generic CORDIC targets applications in a wide range of fields from DSP to matrix math to graphical systems. Overspecialization for one application will reduce the PEs usefulness for others and is to be avoided;

2. Low latency/high throughput - The PE should allow for a high throughput to meet the requirements of modern systems which process an increasing amount of data in a short period of time. The PE should not introduce high latency as would preclude its use in real-time systems;

3. Accurate - The PE must produce results that are accurate to the degree required by the application. Different applications have varying degrees of required accuracy so the PE must be flexible enough to satisfy them;

4. Small area - Modern systems are becoming more integrated. The PE will need to be designed such that a number of them may be included as part of a larger design on a single chip. This requires a minimization of the silicon area used for the PE;

5. Low power - Shrinking technologies, larger chips and portable systems are driving the need for low power components. Low power solutions result in a possible increase in device complexity and extend the battery life of portable devices, and a reduction of overall system costs. Efforts are required to reduce the power consumption of the PE whenever possible;

6. Scalability - As a component for a wide range of systems, the PE design should be scalable. Scaling of the PE will permit various data bitwidths and accuracies as well as area and power requirements. This will allow the PE to meet the requirements of a wide variety of applications.

\subsection{Scaling Factor}

Perhaps one of the most important decisions required is the implementation of the scaling factor compensation. This choice will be examined first as it has significant influences on other choices. The manner in which the desired results are obtained from the out- 
put of the CORDIC process impacts many areas of a design such as latency, hardware requirements and the generation of errors.

Compensation for the scaling factor by pre-scaling the incoming data or product terms performed between iterations, as in equation (2.26), contribute more errors than performing it with additional in-line iterations or post-scaling [24]. Pre-scaling can result in a loss of significant bits at the outset which affects the final accuracy. In-line product terms introduce errors that are not only propagated but magnified by subsequent iterations and product terms. These errors are unacceptable at the output and so strategies are required to compensate for them. The strategies to counter these increased errors have a negative impact on the design as a whole and so, for these reasons, both pre-scaling and in-line product term scaling have been eliminated as acceptable approaches.

Post scaling can be accomplished with a multiplier, with shift and add operations of the form shown in equation (2.25), or with product terms of the form shown in equation (2.27). The drawbacks with using a multiplier are its large area and power consumption which make it an undesirable choice. The use of shift-and-add operations can add a considerable overhead depending on how many operations are required to be performed. For increased accuracy, a linear increase in shift and add operations could be expected. Finally, the use of post scaling product terms can also lead to significant overhead. This overhead again depends on how many terms are required. An additional drawback with using product terms is their sequential nature. For the increased addition operations needed to perform shift-and-add compensation, a tree-style adder could be developed to limit the negative impact on latency. The product terms, however, would have to be performed 
sequentially and would thus have a greater effect on latency. The relative advantage of one method over another is dependent on the scaling factor and level of accuracy desired.

In an effort to quantify the overhead required by the two methods, the case of a 24 bit mantissa is examined. This case corresponds to the requirements of a CORDIC processor using the single precision IEEE floating point format. To achieve 24 bits of accuracy in the result there are roughly 24 iterations required. Assuming the use of Walther's angle basis, the scaling factor following these iterations would be approximately 1.64676 . To compensate for this using shift and add operations would entail the sum of 15 shifted values. The total overhead in hardware would be eight adders each for the $x$ and $y$ datapath, and some combinational logic, using a tree configuration and 3:2 operand compression. The latency overhead would be a delay of three stages of adders and combinational logic. To perform this same compensation using product terms would require more than 12 adders per datapath and would increase the latency.

In-line scaling offers three possibilities for compensating for the scaling factor; product terms, scaling factor steering using repeated iterations and a modified angle basis. The option of product terms has already been ruled out because of the errors produced. It is possible to steer the scaling factor so that a simple shift is required after the completion of the last iteration. One method to achieve this is by repeating some of the normal iterations. The additional $\alpha_{i}$ terms modify the scaling factor and also serve to increase the value of $\theta_{M A X}$. To achieve 24 bits of accuracy in the scaling factor some 22 additional iterations would be required. When compared to the 24 iterations that are required for computational accuracy, this represents an almost $100 \%$ increase in hardware requirements and 
in latency. This is obviously a poorer solution than post-scaling with shift-and-add operations. As the scaling factor is determined by the angles $\alpha_{i}$, another way to steer it is to modify the angle basis. Using Volder's original arc-tangent radix this approach is not achievable without significant loss of accuracy. The problem with the original angle basis for this approach is its lack of flexibility in choosing the angles. Work by Deprettere et al. [16] outlined an angle basis with increased flexibility. The micro-angles are chosen according to equation (2.23) where the parameters $S(m, i), S^{\prime}(m, i)$ and $\eta_{i}$ are chosen such that, among other things, they result in a scaling factor which can be compensated for by a simple shift. For a 24 bit accuracy in the scaling factor this can be achieved with as few as seven additional adders for each datapath and an increase in latency of seven adders. This solution uses fewer adders than post-scaling with shift-and-add operations but results in a larger increase in latency.

Post scaling with shift-add operations and inline steering with a double shift angle basis are both acceptable solutions, each with their own strength. The shift-add operations require more hardware while the double shift angle basis adds more latency. A selection of which method to use must, therefore, be based on their advantages and disadvantages in other areas and the overriding design goals of the generalized PE.

\subsection{Number System}

The choice of the internal number format also has a significant impact on the selection of the CORDIC method. The input and output of the CORDIC PE is typically in floating point format in order to provide a greater range of operation. The format of the data 
internal to the PE is an independent decision. The iteration equations involve addition as the computational operation. This makes the selection of floating point numbers for the internal format a poor one as each stage would require an alignment and a normalization operation. The disadvantages of the extra hardware requirements and increased latency are not offset by any significant advantage and so this choice can be eliminated.

Considerable research has been conducted on the use of redundant number systems in the CORDIC process. The latency of the process is determined principally by the time it takes to perform the iterations. The large number of iterations means that any reduction in the execution time of a single iteration leads to a larger overall benefit. The major operation in each iteration is the addition, and so the focus has been on reducing the time it requires. Redundant number systems such as signed digit and carry-save result in faster additions as their execution time is no longer governed by the timing in the carry chain. It is, therefore, possible to design extremely fast CORDIC PEs using redundant number systems. This benefit, however, comes with a considerable price in area and power consumption. The first cost comes with the number format itself. Redundant number systems require extra bits in the form of a carry vector in carry-save format or sign bits in signed digit format. The number of extra bits required represents a $100 \%$ increase in datapath width. For a pipelined architecture this requires significantly more sequential components. The second cost comes with the fact that the sign of a number is no longer easily determined. The application of the micro-angle at each iteration depends on the sign of either $y$ or $z$ and so more elaborate algorithms are required when the signs are not known. This was presented in section 2.3.3 along with various proposed methods to deal with it. 
An examination of the proposed algorithms reveals that the hardware requirements are, on average, double the requirements of the basic CORDIC method. Takagi et al. proposed in [7] and [8] to perform each iteration in two parts. This would require the same number of iterations but double the number of adders per iteration. In a second method they proposed, extra iterations correct for rotations when the sign is unknown. The number of extra iterations needed for a 24 bit accuracy is in the order of eight, but that depends on how much effort is devoted to determining the sign. Duprat and Muller's [11] branching CORDIC performs each iteration in two separate modules and so requires double the architecture components. For applications where the PE is to be used as part of a larger design module or where many PEs are required on a single chip, such as in systolic arrays, then the use of redundant number systems will be at a serious disadvantage.

A third choice for the internal number format is block floating point format as already presented in section 2.6. The selection of the block floating point number format does not lead to any increase in hardware requirements as it is analogous to the fixed point format that was originally used [4].

An additional aspect concerning number format must be addressed. As presented in section 2.6, the format of the $z$ datapath can be in either fixed point or floating point format. Having the $x$ and $y$ inputs in floating point format does not necessitate a floating point format for the angle input. CORDIC methods which allow floating point angle representation must be adaptive in order to accommodate the large range of angles. As outlined, the methods of adaptation thwart attempts to steer the scaling factor to a known constant which can be easily dealt with. The compensation of the scaling factor becomes data 
dependent and requires increased efforts to perform. It becomes evident from an analysis of current research into applications of the CORDIC algorithm that this fine resolution in the angle format is not required. Of all the applications researched in the fields of DSP, general and matrix math processors and graphical systems none were found which implemented a floating point angle format. Hekstra and Deprettere developed a floating point angle algorithm for circular and linear coordinate systems [35] but had not yet developed an equivalent algorithm for the hyperbolic. Allowing the angle to be in floating point format has a significant negative impact on other aspects of the CORDIC method, principally with the compensation of the scaling factor, and with no appreciable benefits for the target application. Therefore, it will not be implemented here as such.

\subsection{Accuracy}

Errors introduced during the CORDIC process are, for the most part, unavoidable. Input value rounding error can be eliminated if the width of the datapath is equal to the width of the input data but the remaining errors exist to varying degrees in all implementations.

Angle approximation error caused by a finite number of iterations can be reduced by increasing the number of iterations. Rounding error caused by a finite datapath width is introduced during each iteration and so increases with the number of iterations. At the same time as more iterations are included in the CORDIC process to reduce the angle approximation error, the rounding error increases. The number of iterations should, therefore, be minimized while maintaining an acceptable level of angle approximation error. 
The impact of the rounding error can be lessened by appending a suitable number of bits to the least significant end of the datapaths. When performing an addition using a truncated operand, the upper bound on the error introduced is 1 units of the last place (ulp) with the statistical average being half of that. If $\left\lceil\log _{2}(n)\right\rceil$ bits are added to the least significant part of the datapath then the errors introduced by $n$ addition operations will be restricted to the extra bits. These bits can then be eliminated from the final result with no loss of accuracy. The downside of this solution is an increase in hardware costs due to the wider datapath. It is possible to reduce the number of additional bits required by reducing the number of iterations, but it is also possible to limit the level of error per addition operation. If additional bits are retained when the shifted operand is formed, and they are subsequently used in the addition operation, then it is possible to limit the error introduced to 1/2 ulp. This halves the maximum possible error from the operations and reduces the number of extra bits required to $\left\lceil\log _{2}(n / 2)\right\rceil$. Reducing the datapath width and including the additional bits in the adders result in savings in terms of silicon area for any implementations where $n>4$. This topic is covered in more detail in section 4.4 .

Scaling factor error can be reduced to an acceptable level by ensuring that the steps taken to compensate for the scaling factor are themselves accurate to the required number of bits. This will ensure that the compensation operations do not introduce errors after the iterations are complete. 


\subsection{Latency and Throughput}

With approximately 24 serial iterations required to perform Volder's original CORDIC process, to compute a set of results with 24 bits of accuracy, it is clear that moden applications of the algorithm require strategies to increase throughput. A common method for increasing throughput is by pipelining the datapath. This introduces latency as the pipeline must fill before any results are obtained but it can significantly increase throughput. The increase in throughput is governed by how many pipeline stages are implemented, more pipeline stages result in higher throughput. This increase in throughput is achieved at the expense of area and power as registers are required between pipeline stages to isolate their respective portion of the datapath.

The expense in terms of area, power and latency, associated with pipelining can be controlled by limiting the number of stages implemented and the width of the datapaths. The use of a redundant number system leads to a higher cost for pipelined implementations as the datapath is roughly doubled. This represents a serious drawback to the use of such number systems.

Other possible CORDIC methods such as the Partitioned-Hybrid algorithm using a mixed ATR angle basis suffer from an inability to be advantageously pipelined. In the hybrid angle basis, as expressed in equation (2.19), the least significant iterations can be performed in a parallel fashion using merged CORDIC equations. This method can lead to a reduced latency without impacting throughput. As part of these merged iterations, the iteration operations become more complicated making the implementation of multiple pipeline strategies difficult. The operations of the merged iterations would have to con- 
form to the operation time budget of the other pipelined stages and this is not easily achievable.

\subsection{Selection Decision}

To summarize the key findings from the previous discussion:

1. Compensation for the scaling factor is best done with post scaling shift-and-add operations or with a double shift angle basis;

2. The floating point number format will be used for the input and output of $x$ and $y$ in order to benefit from the dynamic range and accuracy. A fixed point format will be used for the input and output of $z$ as the increased accuracy of the floating point format is not required;

3. The fixed point (block floating point) number representation will be used for all internal computations to reduce the computational overhead associated with alignment and normalization;

4. The internal datapaths will be padded at the least significant end to capture errors;

5. All additions will be performed in such a way as to minimize the errors generated; and,

6. The chosen method must be capable of being pipelined to varying degrees to meet the wide range of design requirements.

As a result of these findings and the design goals, the number of CORDIC methods which are possible choices is reduced to two. This first possibility is to use iteration equations in their unmodified form along with shift and add operations to perform post-scaling. The second possibility is to use iteration equations modified to allow for the double shift angle basis and reduce the compensation to a simple shift operation. The two options require about the same additional hardware to compensate for the scaling factor but the second option incurs less of a penalty in pipelined implementations. The first option of 
post scaling requires registers between the stages of adders, seven in total for a 24 bit implementation, while the second option requires registers for the additional iteration stages, two in a 24 bit implementation. The second option also benefits in other ways. Recall that the double shift angle basis provides an increased range of angle convergence and allows for coordinate system independent execution times. When taken as a whole, the benefits of the double shift angle basis outweigh the small increase in latency that it exhibits as compared to the post scaling shift-and-add option. The double shift CORDIC algorithm is, therefore, the basis for the generic CORDIC PE and will be examined in detail in the following chapter. 


\section{CHAPTER 4}

\section{ALGORITHM IMPLEMENTATION}

The selection of the double shift version of the CORDIC algorithm still leaves many design issues to deal with. These issues include the input and output data format, the minimization of rounding errors, reduction of output errors and the selection of the iteration parameters. These issues and the details of the generic CORDIC PE implementation will be discussed in this chapter. The final section is a presentation and discussion of the implementation accuracy obtained through simulation.

\subsection{Input / Output Data}

The primary inputs to the CORDIC process are the two coordinates $x$ and $y$ as well as the angle. The $x$ and $y$ inputs are in floating point format, while the angle is in fixed point format, but the choice of bitwidths remains. The choice of bitwidths for $x$ and $y$ was made after a thorough investigation of applications that utilized the CORDIC algorithm to determine what the requirements were. Most of the applications utilized a range of widths from a low of 17 bit to a high of 32 bit floating point. The choice was made to implement four widths within this range corresponding to the various implementations that were found in the investigation. The dynamic range of floating point numbers is: 


$$
D R_{f l p}=2 \cdot 2^{\left(2^{e}-1\right)}
$$

where $e$ is the number of bits in the exponent. This may also be expressed in decibels as $20 \log \left(D R_{f f p}\right)$ [36]. The four selected bitwidths and their dynamic range are shown in Table 4.1.

\begin{tabular}{|c|c|c|c|}
\hline $\begin{array}{c}\text { Format } \\
\text { Choice }\end{array}$ & $\begin{array}{c}\text { Mantissa } \\
\text { Width }\end{array}$ & $\begin{array}{c}\text { Exponent } \\
\text { Width }\end{array}$ & $\begin{array}{c}\text { Dynamic } \\
\text { Range }\end{array}$ \\
\hline 1 & 12 & 5 & $192 \mathrm{~dB}$ \\
\hline 2 & 16 & 5 & $192 \mathrm{~dB}$ \\
\hline 3 & 18 & 6 & $385 \mathrm{~dB}$ \\
\hline 4 & 24 & 8 & $1541 \mathrm{~dB}$ \\
\hline
\end{tabular}

TABLE 4.1. X/Y Input Output Formats

The mantissas are in 2's complement notation for reasons of computational efficiency while the exponents are biased values. A comparison between signed magnitude format and 2's complement notation is shown in Figure 4.1. The amount of the exponent bias depends on the number of exponent bits $e$ and is equal to $+2^{e}-1$. This corresponds to the biased exponent scheme used in the IEEE floating point standard. Unlike IEEE floating point numbers there are no hidden 1's included in the mantissa and, since the number is in 2's complement form, what would have been the sign bit is now included as part of the mantissa to maintain the same range. The positioning of the binary point in the mantissa is shown in Figure 4.1b where the MSB of the mantissa is bit $M_{b-I}$. This format allows mantissa values from -2 to $2-2^{-b+2}$ to be represented in 2 's complement notation. 
The angle value $z$ is in fixed point format and in 2's complement notation. In order to represent the range of values $\pm \pi$, as required by the desired zone of convergence, while

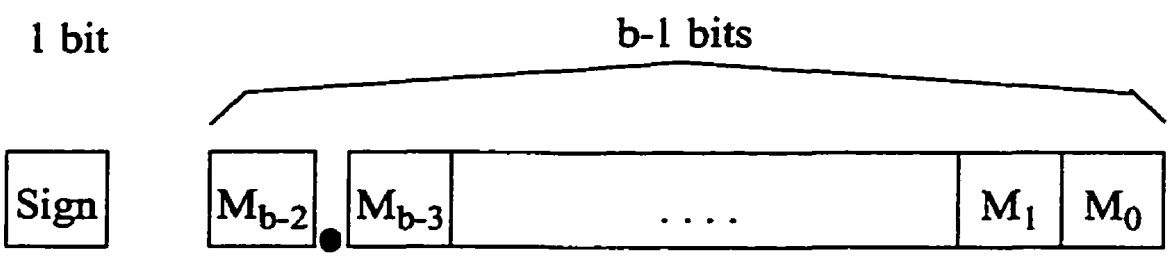

a) Signed Magnitude

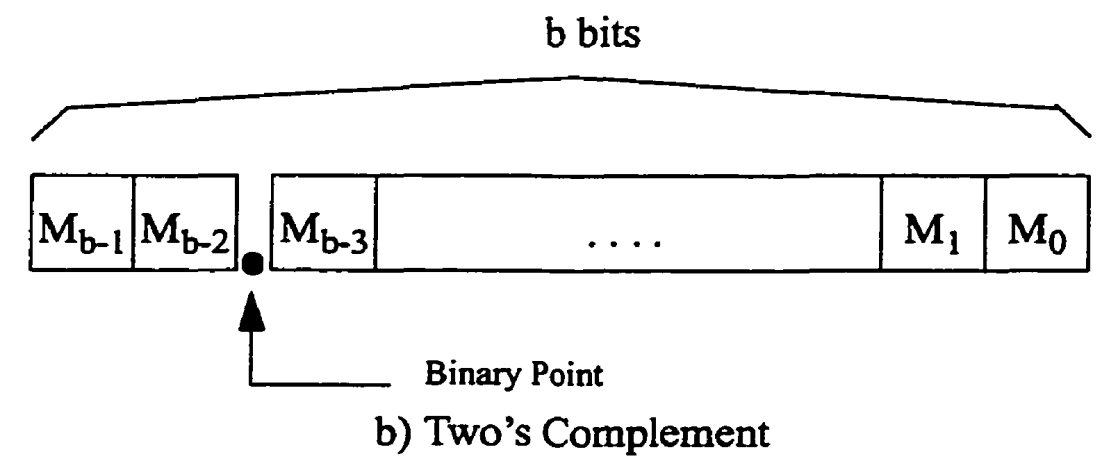

FIGURE 4.1. Mantissa Formats

still retaining the same degree of accuracy as the $x$ and $y$ results, the $z$ datapath is required to be one bit wider than the $x$ and $y$ datapath. The $x$ and $y$ mantissas permit a relative resolution of $2^{-b+2}$ and can encode values with an upper and lower bound of \pm 2 . To achieve this same resolution in fixed point format, while expanding the bounds to \pm 4 to accommodate values of $\pi$, requires an additional bit to the left of the binary point. The positioning of the binary point in the angle value is shown in Figure 4.2 , where the MSB is $Z_{b}$ and $b$ refers to the mantissa width of $x$ and $y$. 


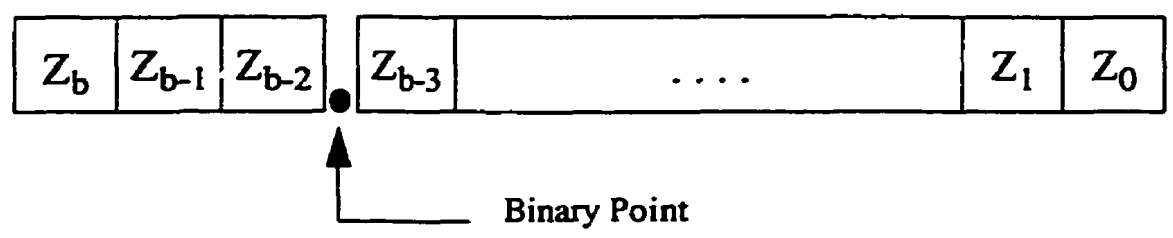

\section{FIGURE 4.2. Input/Output Angle Format}

The generic CORDIC PE also allows for the alternative representation of $z$. Recall the fact that the angle $\theta$ can also be represented as a set of rotation directions $\sigma_{i}$ according to equation (4.2). Using the set of rotation directions allows for the rotation over a previously decomposed angle, as occurs in matrix math applications. When rotating a vector by an angle determined by the first vector element, the rotation directions $\sigma_{i}$ are computed by a vectoring $\mathrm{PE}$ and need not be recomputed for the rotation of the remaining vector elements. The $\sigma_{i}$ values can be output from the vectoring PE so that the dependent rotations can begin with a minimum of delay.

$$
\theta=\sum_{i=0}^{n-1} \sigma_{i} \cdot \alpha_{i}
$$

Depending on the application, the use of the direction set notation saves both types of PEs from having to perform the angle update iteration equations, thus saving area and power. The $z$ output of a vectoring operation is simply an encoded version of the angle which must be decoded by subsequent rotation operations. This results in an increased delay and decreased computational efficiency. 


\subsection{Rounding}

The IEEE standard for rounding specifies that an arithmetic operation must produce a result as if it had been computed exactly and then rounded. For floating point addition the computation of exact results requires the use of all shifted out bits although this is seldom required in practice. Rounding is not required for cases of addition where there are no shifted operands as the result is already exact. In cross addition, as occurs in the pipeline stages of the CORDIC, where there is no post normalization shift, it is sufficient to retain only two extra bits from the shifted out numbers, called the round (R) and the sticky (S) bits, to perform a round to the nearest even scheme. This scheme is preferred to a rounding scheme where all possible bit combinations lead either to rounding up or down without regard for the even/odd condition, as it does not bias the result.

\begin{tabular}{|c|c|c||c|c|c|}
\hline Significand & $\begin{array}{c}\text { Rounded } \\
\text { Result }\end{array}$ & Error & Significand & $\begin{array}{c}\text { Rounded } \\
\text { Result }\end{array}$ & Error \\
\hline \hline $\mathrm{X} 0.00$ & $\mathrm{X} 0$. & 0 & $\mathrm{X} 1.00$ & $\mathrm{X} 1$. & 0 \\
\hline $\mathrm{X} 0.01$ & $\mathrm{X} 0$. & $-1 / 4$ & $\mathrm{X} 1.01$ & $\mathrm{X} 1$. & $-1 / 4$ \\
\hline $\mathrm{X} 0.10$ & $\mathrm{X} 0$. & $-1 / 2$ & $\mathrm{X} 1.10$ & $\mathrm{X} 1 .+1$ & $+1 / 2$ \\
\hline $\mathrm{X} 0.11$ & $\mathrm{X} 1$. & $+1 / 4$ & $\mathrm{X} 1.11$ & $\mathrm{X} 1 .+1$ & $+1 / 4$ \\
\hline
\end{tabular}

TABLE 4.2. Round to Nearest - Even Scheme

A non-biased rounding scheme is one where the sum of the rounding errors of all possible rounding decisions is zero. The round to nearest even scheme is shown in Table 4.2 along with the errors that are caused by the rounding. In Table 4.2 the upper bits are represented by an $\mathrm{X}$ and a period separates the $\mathrm{R}$ and $\mathrm{S}$ bits from the remainder of the significand bits.

The generation of the extra bits is shown graphically in Figure 4.3. As stated, this scheme requires the retention of the two additional bits, the round bit and the sticky bit. 
The round bit is simply the bit that was immediately to the right of what becomes the least significant bit of the significand while the sticky bit is the logical OR of all the bits that were to the right of the round bit and have now been shifted out. The significance of the sticky bit is to indicate whether the shifted operand is exact or whether some quantity was lost during the shift. In floating point addition where there is a variable length shift required to align the operands, the generation of the sticky bit can be difficult. In the CORDIC case the magnitude of the shift is known apoiri, it's a constant for each adder of each pipeline stage, so the sticky bit can be quickly and easily generated. In the

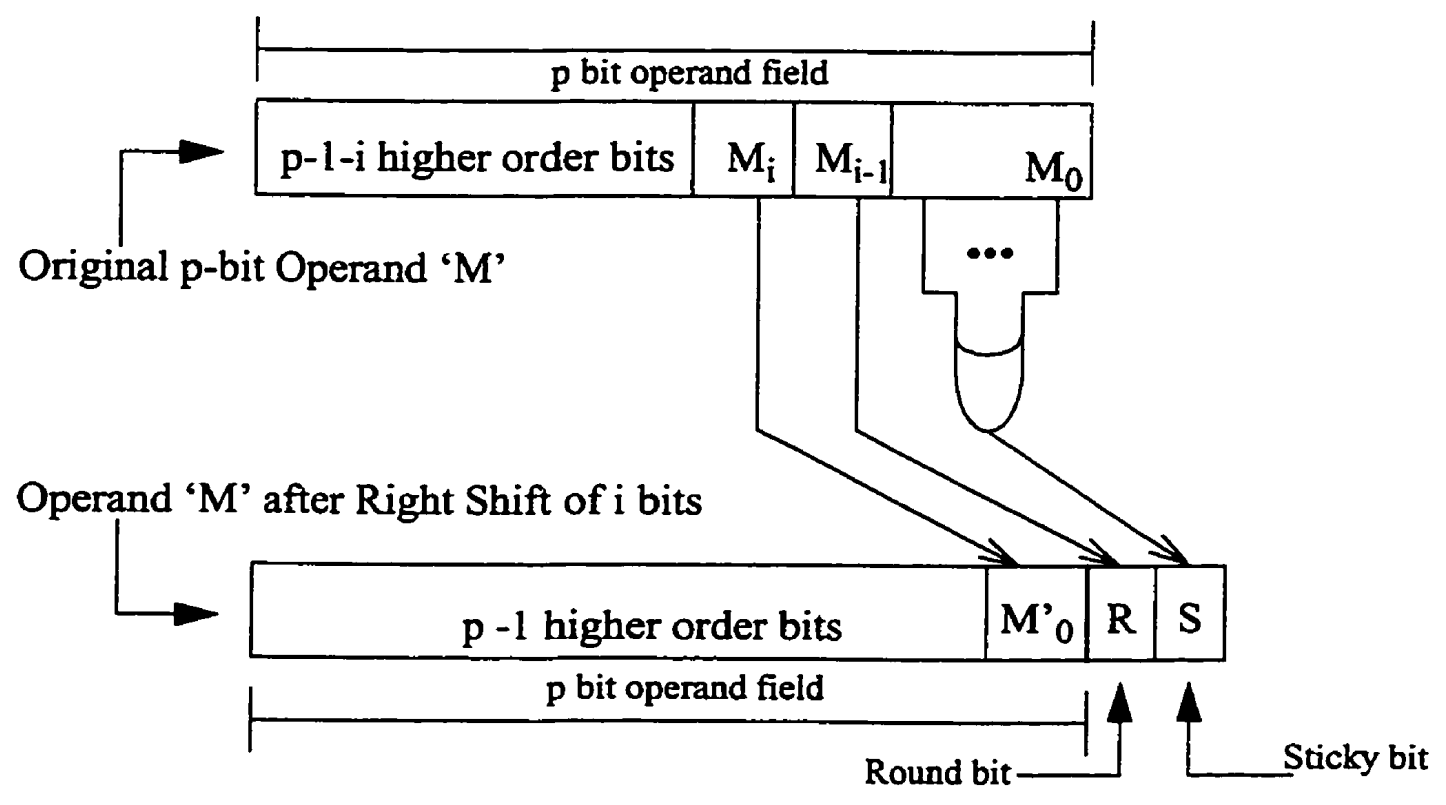

FIGURE 4.3. Generation of Round and Sticky Bits

cases where the right shift is by zero or one bit, the least significant part of the operand is padded with zeros to generate the required number of bits. 
Once the addition/subtraction has been performed the resultant $N$ has the form shown in Figure 4.4. The resultant includes the solution for the two additional bits, $R$ ' and

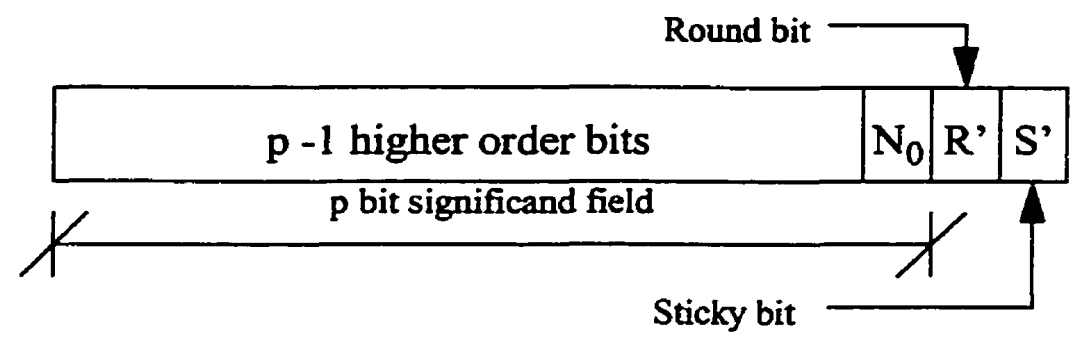

FIGURE 4.4. Resultant with Rounding Bits

$S^{\prime}$, which are used to evaluate the rounding decision. As can be seen from Table 4.2 the rounding decision may necessitate a conditional addition of $1 / 2 \mathrm{ulp}$, although this requirement can only be evaluated after the $N_{0}, R^{\prime}$ and $S^{\prime}$ digits have been computed. Table 4.3 lists the various possibilities.

\begin{tabular}{|c|c|c|c|l|}
\hline $\begin{array}{c}\mathbf{N}_{\mathbf{0}} \\
\text { (LSB) }\end{array}$ & $\begin{array}{c}\text { R' } \\
\text { (Round } \\
\text { bit) }\end{array}$ & $\begin{array}{c}\text { S' } \\
\text { (Sticky } \\
\text { bit) }\end{array}$ & $\begin{array}{c}\text { Addition } \\
\text { of 1 at } \mathbf{N}_{\mathbf{0}} \\
\text { position }\end{array}$ & \multicolumn{1}{|c|}{ Remarks } \\
\hline 0 & 0 & 0 & No & No rounding - result is exact \\
\hline 0 & 0 & 1 & No & No rounding - result is not exact \\
\hline 0 & 1 & 0 & No & Tie case - even result is chosen \\
\hline 0 & 1 & 1 & Yes & Round to nearest adding 1/2 ulp \\
\hline 1 & 0 & 0 & No & No rounding - result is exact \\
\hline 1 & 0 & 1 & No & No rounding - result is not exact \\
\hline 1 & 1 & 0 & Yes & $\begin{array}{l}\text { Tie case - round to nearest even by } \\
\text { adding l/2 ulp }\end{array}$ \\
\hline 1 & 1 & 1 & Yes & Round to nearest adding 1/2 ulp \\
\hline
\end{tabular}

\section{TABLE 4.3. Injection of 1 at LSB for Rounding}

In the round to nearest even scheme, there is no rounding required when the $R$ ' bit is zero. The $S^{\prime}$ bit indicates whether the rounded result is exact or not. If the $S^{\prime}$ bit is a zero then the result is exact and vice-versa. If both $R^{\prime}$ and $S^{\prime}$ bits are set to one then the addition 
of $1 / 2$ ulp is required. If $R^{\prime}$ is one and $S^{\prime}$ is zero rounding is required only if $R_{0}$ is one. The rounding up condition is then given by:

$$
R N D=R^{\prime} S^{\prime}+R^{\prime} \bar{S}^{\prime} N_{0}=R^{\prime}\left(S^{\prime}+N_{0}\right)
$$

\subsection{CORDIC Addition/Subtraction}

The modified CORDIC equations require summations of two and three operands for the single shift and double shift iterations respectively. The two operand summation is straight forward, however, there are options when dealing with the three term sums.

For this application it is not feasible to attempt to add all three terms together in one summing operation as both of the operators may be subtractions and require a two's complement of the operands to be performed. The difficulty arises in that the operation required is based on the previous result and is, therefore, not known in advance. In the cases where both of the operations are subtractions a carry-in of two is required as part of the 'invert and add one' two's complement method. As part of the overall summing operation, this could cause a carry-in of two at any bit position and thus complicates the three operand sum so much as to make it impractical or at least sub-optimal.

The three operand sum can be accomplished with two separate two operand addition/subtractions. According to the associative and commutative properties of addition/ subtraction, the operations can be performed in different ways as shown in Figure 4.5. If two separate sums are to be used, then the first option is to sum the non-shifted operands with a shifted one followed by a sum of the result with the remaining shifted operand. This approach has the disadvantage that both sum operations involve a shifted operand and thus 
require rounding be performed. This leads to two sums, each of which contributes a maximum of $1 / 2$ ulp to the overall error.

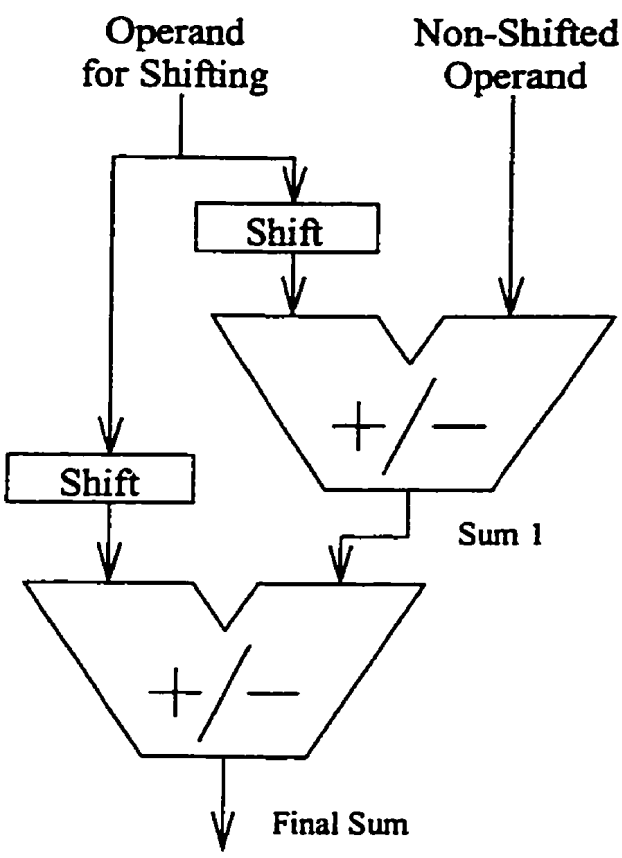

a) Option One

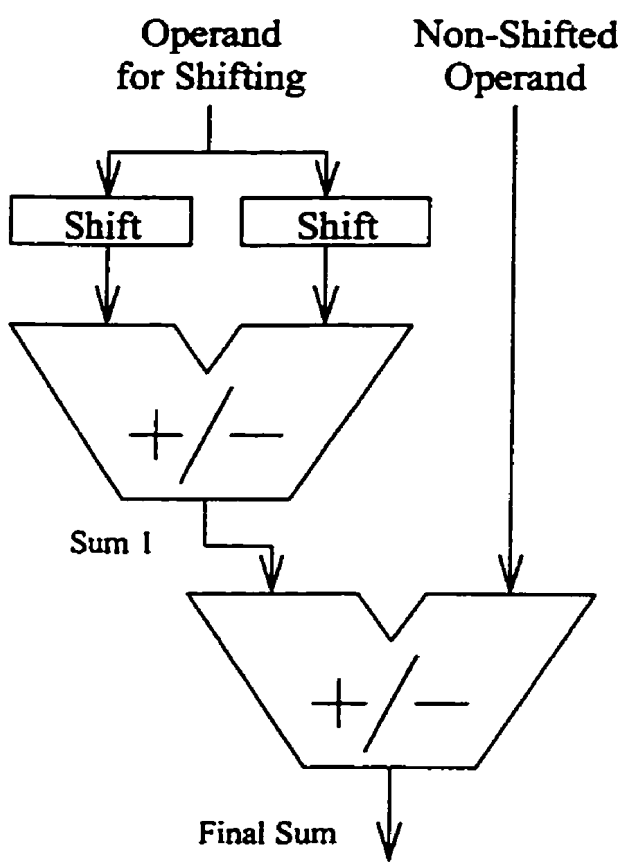

b) Option Two

FIGURE 4.5. Three Term / Two Adder - Addition Options

The second option is to sum the two shifted operands first and then sum the result with the non-shifted operand. In this approach, the second sum does not require rounding as the only shifted operands occur in the first sum, which can perform the required rounding. Consider the case that all $p+2$ bits from the result of the first sum form the second operand to the second sum. The first operand, as a non-shifted operand, will have its $R$ and $S$ bits set to zero. The $R$ and $S$ bits of the second sum are, therefore, determined by the second operand only. No new information has been contributed for the rounding decision and so the same result will be produced as could have been produced if the rounding decision was made after the first sum. It is clear that leaving the rounding decision to the second 
summation leads to a sub-optimal solution where the second adder would have a larger operand length than required.

A second advantage of summing the two shifted operands together is a reduction of toggling within the adder. As the operands are the result of two different shifts of the same number, there will be a common set of upper bits which are sign extended during the shift operation. This allows for a reduction in transition activity as well as allowing for logic optimization. The addition of shifted operands is a known method for power reduction and is outlined in detail in [37]. The second arrangement of operands thus benefits from a reduction in area and power and only introduces a maximum total error of $1 / 2$ ulp per iteration. The addition of two shifted operands, however, makes rounding Method One unsuitable and so another method is presented in section 4.5.2 for this case.

\subsection{Requirement for Rounding}

When right shifting operands, there are options as to how the number is formed and how the subsequent operation is performed. These choices affect the architecture as well as the accuracy of the result and so each possibility must be examined to find an optimal solution for the general cases that the generic CORDIC deals with.

Option One is to perform rounding as presented in section 4.2. With rounding a maximum error of $1 / 2$ ulp is generated per operation. To prevent this error from affecting the final results, it is possible to pad the least significant portion of the number with a suitable number of bits so that the error is restricted to the additional bits. The number of bits required, irrespective of the source of the error, is $\left\lceil\log _{2}\right.$ (total error) $\rceil$. For rounding in the 
generic CORDIC, where there is only one rounding operation per stage, the number of padding bits required is $\left\lceil\log _{2}(\right.$ stages /2) $]$.

Option Two is to perform truncation. Truncation of a binary number results in a maximum error of 1 ulp in the resulting number. This occurs when all truncated digits are ones. If truncation were to be used in the CORDIC PE then the shifted numbers would each contribute their respective 1 ulp error to any mathematical operation. This would result in a maximum error of 2 ulp for additions with two shifted operands and 1 ulp for two operand subtractions and other two operand additions. This means a maximum error of 2 ulp for double shift iterations and 1 ulp for single shift iterations. The elimination of the $R$ and $S$ bits from the shifted numbers would result in smaller adder/subtractors by two bits. To maintain an accurate result to the required number of digits would require the least significant portion of the number be padded to capture this increase in errors. With rounding the errors are a maximum of $1 / 2$ ulp irrespective of iteration type and so truncation represents an increase in the total maximum error by a factor of between two and four depending on the relative number of single and double rotation iterations. The increase in error results then in two bits of padding in addition to the padding required in Option One. This increased data path widens all adders/subtractors by two bits as well as requiring additional registers for each pipeline stage. This represents a significant cost in area and power consumption and so rounding is preferable to truncation in this case.

Option Three is a modified form of truncation whereby the shifted numbers retain the $R$ and $S$ bits as in the rounding scheme presented, however, truncation is performed in lieu of rounding at the end of the mathematical operation. This results in the generation of 
a maximum error of 1 ulp for each operation involving shifted operands. The iterations then produce an error of up to 1 ulp each, irrespective of type, as they both involve shifted operands. As in the case of Option Two, using full truncation, this error is more than the error in the rounding case and requires additional bits of error padding. In this case the error is double the maximum error with rounding and so one additional bit of padding is sufficient. Again, this increase represents a wider data path which requires wider adder/ subtractors as well an additional register for each pipeline stage. The increase in additional components is less than for full truncation, however, this still represents an increase in area and power. The increase might be acceptable if it was coupled with a significant speedup due to the elimination of the rounding logic, however, this does not occur.

Rounding then becomes necessary in order to achieve a reduction in area and power consumption for the design while maintaining the required level of accuracy.

\subsection{CORDIC Rounding}

In the CORDIC iterations there are three operand sums during the first set of iterations and two operand sums for the remainder. For the two operand sums where only one operand is the result of a shift, the rounding method is presented as Method One. For the case of a three operand sum where two operands are the result of shift operations, and where it is desirable to sum the two shifted operands, the rounding method is presented as Method Two. 


\subsubsection{Method One - One Shifted Operand}

This rounding scheme is used in conjunction with two operand sums where only one of the operands is the result of a shift towards the LSB. In this scheme the two extra bits are retained, as discussed, when the shifted value is produced. The operand which is not shifted is appended with zeros for the $R$ and $S$ bits in order to have an operand length equal to the shifted operand. If the negative of the second operand is required, i.e. a subtraction needs to be performed, then the second operand can be bitwise inverted and the $S$ bit of the first operand set to a one. This effectively results in a two's complement operation without any extra computation. Assuming an original operand length of $p$ bits, the two operands $A$ and $B$ are shown in Figure 4.6, where $A_{O}$ is a zero for addition and a one for subtraction.

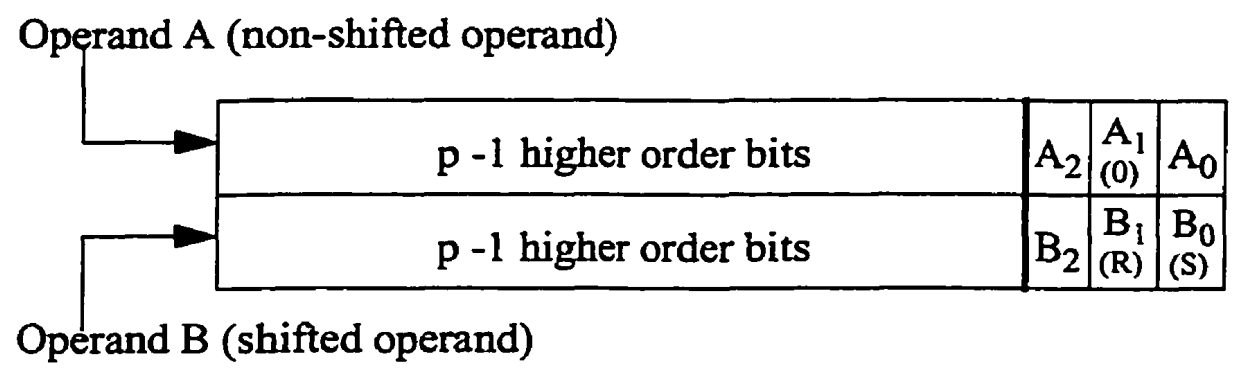

FIGURE 4.6. Pre-addition Operands - Method One

The two's complement addition is dealt with in two blocks as shown in Figure 4.7. The Upper Block is formed from the most significant $p-1$ bits while the Lower Block is the least significant three bits. The addition in the Upper Block is best performed using a conditional sum or carry select type architecture while the addition of the lower block can be performed using a ripple architecture due to its small size. The architecture of the Upper Block enables the selection of the proper result once the addition of the Lower Block has 
been performed and the rounding decision made without additional delay. In combination with the conditional sum architecture of the Upper Block, the Lower Block's architecture is not a significant issue as it can be slow without affecting the overall timing. This provides a means of performing no-overhead rounding. The addition of the Lower Block is performed and, based on the results obtained, the LSB of the result is set and the appropriate Upper Block result is selected based on the carry out of the Lower Block.

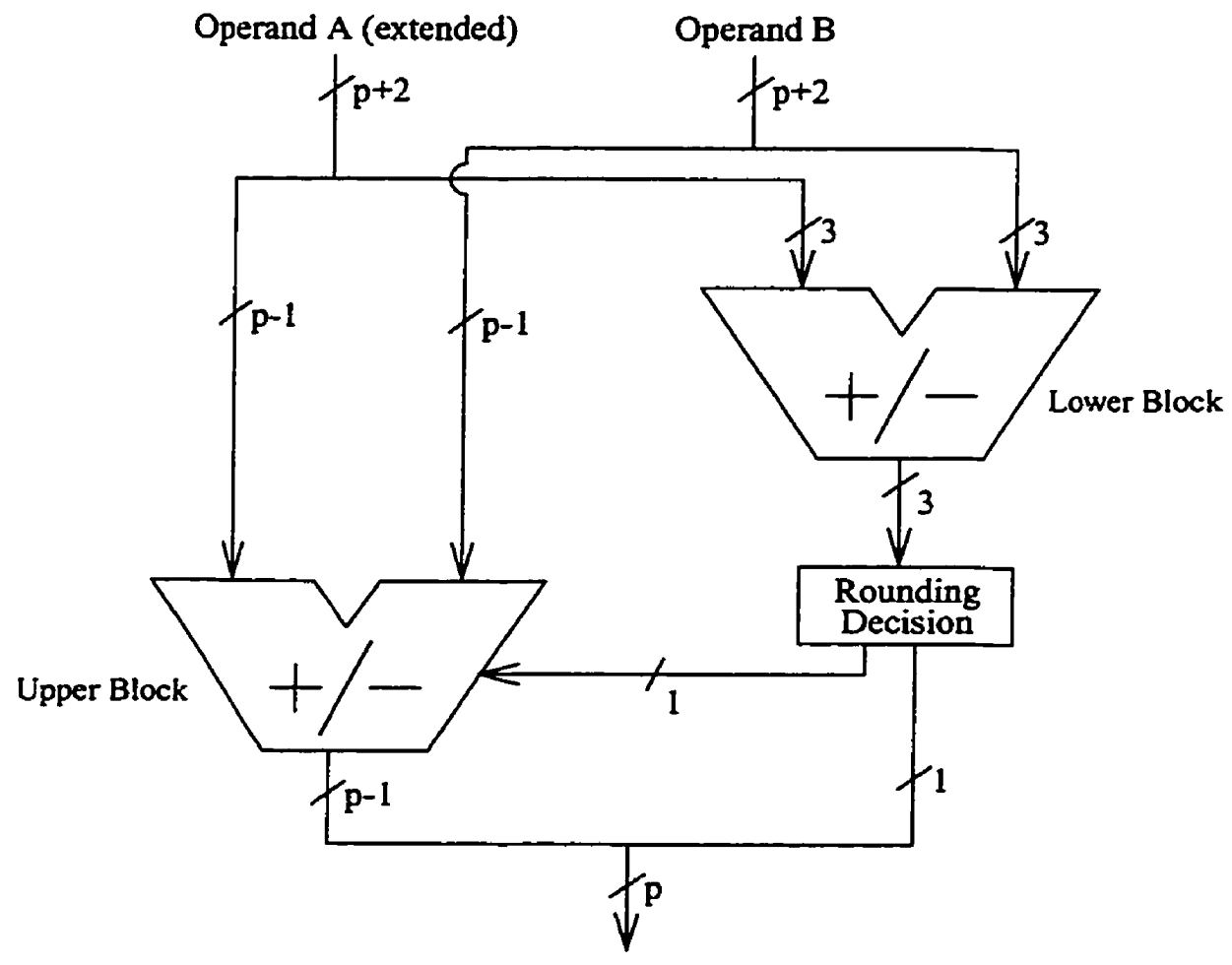

FIGURE 4.7. CORDIC Rounding - Method One

An examination of the conditions that lead to a carry out of 1 from the Lower Block due to the addition operation and those that lead to a carry out of 1 based on the rounding decision reveals that they are mutually exclusive. This occurs since the $A_{l}$ bit is 
guaranteed to be a zero. Any valid combination of inputs that leads to a carry out from the addition operation also produces results where any addition of 1 ulp due to rounding does not propagate out of the Lower Block. This is shown in Table 4.4 where $A$ and $B$ are the two operands, $C_{\text {out }}$ is the carry out from the addition, $R$ is the result of the sum and $N_{O}$ is the LSB to be appended to the result of the Upper Block. Where rounding is required it is possible to add the 1 ulp without forcing a second carry out.

\begin{tabular}{|c|c|c|c|c|c|c|c|c|c|c|c|}
\hline $\mathbf{A}_{\mathbf{2}}$ & $\mathbf{A}_{\mathbf{1}}$ & $\mathbf{A}_{\mathbf{0}}$ & $\mathbf{B}_{\mathbf{2}}$ & $\mathbf{B}_{\mathbf{1}}$ & $\mathbf{B}_{\mathbf{0}}$ & $\mathbf{C}_{\mathbf{0 u t}}$ & $\mathbf{R}_{\mathbf{2}}$ & $\mathbf{R}_{\mathbf{1}}$ & $\mathbf{R}_{\mathbf{0}}$ & $\mathbf{N}_{\mathbf{0}}$ & Remarks \\
\hline \hline 0 & 0 & 1 & 1 & 1 & 1 & 1 & 0 & 0 & $\mathbf{0}$ & 0 & \\
\hline 1 & 0 & 0 & 1 & 0 & 0 & 1 & 0 & 0 & 0 & 0 & \\
\hline 1 & 0 & 0 & 1 & 0 & 1 & 1 & 0 & 0 & 1 & 0 & \\
\hline 1 & 0 & 0 & 1 & 1 & 0 & 1 & 0 & 1 & 0 & 0 & \\
\hline 1 & 0 & 0 & 1 & 1 & 1 & 1 & 0 & 1 & 1 & 1 & Rounding Required \\
\hline 1 & 0 & 1 & 0 & 1 & 1 & 1 & 0 & 0 & 0 & 0 & \\
\hline 1 & 0 & 1 & 1 & 0 & 0 & 1 & 0 & 0 & 1 & 0 & \\
\hline 1 & 0 & 1 & 1 & 0 & 1 & 1 & 0 & 1 & 0 & 0 & \\
\hline 1 & 0 & 1 & 1 & 1 & 0 & 1 & 0 & 1 & 1 & 1 & Rounding Required \\
\hline 1 & 0 & 1 & 1 & 1 & 1 & 1 & 1 & 0 & 0 & 1 & \\
\hline
\end{tabular}

TABLE 4.4. Carry Out Generated by Addition

\subsubsection{Method Two - Two Shifted Operands}

In the case where both of the operands are obtained by shifting, rounding Method One will not work as the condition of mutual exclusion between the addition carry out and the rounding carry out from the lower block is no longer valid. This can be seen from the fact that the $R$ and $S$ bits of the first operand are no longer guaranteed to be 00 or 01 . In the case of operands of the Lower Block both being 111 (or 110 and 111 along with a carry in 
for subtraction) there can be a carry out generated from the addition operation and another required by the rounding decision. This fact requires a different approach for dealing with no-overhead rounding.

The method again splits the operation up into an Upper and a Lower Block as shown in Figure 4.8. The method is also based on the fact that if there can be a carry in from both sources, then the combined carry-in to the Upper Block will be equivalent to two. This can be seen from the fact that a carry will always be generated from the addition and a second carry is generated by the rounding decision. In the case where a carry in of two is not possible than the carry in to the Upper Block will be a zero or a one. There are a

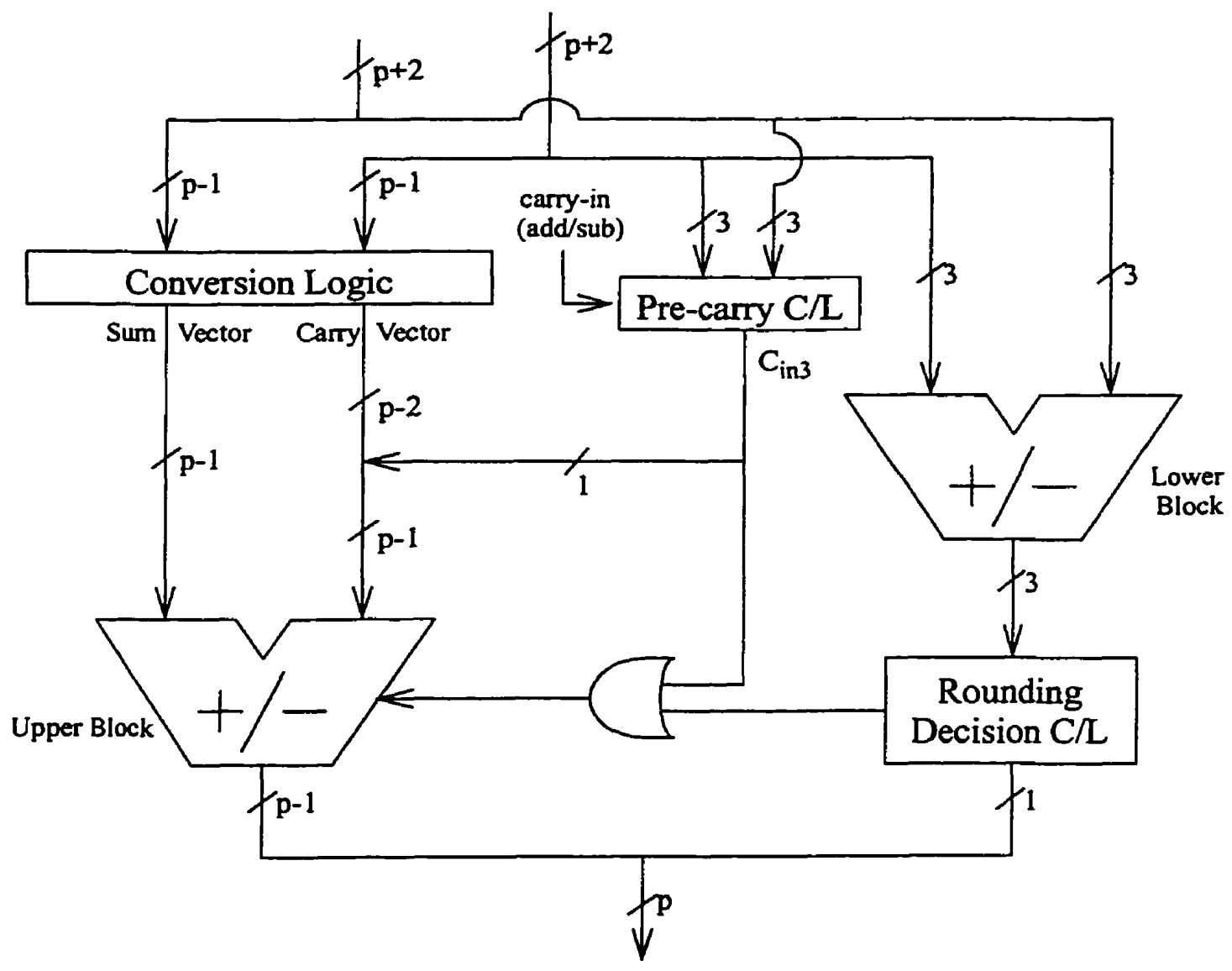

FIGURE 4.8. CORDIC Rounding - Method Two 
total of 72 different scenarios for the Lower Block operation based on the 36 possible combinations of operands and the two operations, addition and subtraction. Of these 72 cases, however, only three lead to a carry of two. The first two occur when both operands have a binary value of 111 and the operation is either addition or subtraction. The third is when the operands have a binary value of 111 and 110 in a subtraction operation and occurs due to the carry in of one required for the 2's complement operation. Since the cases where a carry in of two is required are easily determined and constitute a small subset of all possible operand combinations, it is fairly simple to ensure that the Upper Block computes the results for the proper carry. This is performed in the Pre-Carry Combinational Logic block of Figure 4.8 where the decoding logic for these conditions is:

$$
C_{i n 3}=A_{2} \bullet A_{1} \bullet B_{2} \bullet B_{1} \bullet\left(A_{0} \bullet B_{0}+C_{i n 0} \bullet\left(A_{0}+B_{0}\right)\right.
$$

In cases where a carry in of two is required, it is possible to perform a pre-carry for the Upper Block. This pre-carry takes the form of an addition of 1 ulp to the operands of the Upper Block. In this way one of the two carries is included in the operands and the second can be considered as a normal carry-in signal. With this method the number of operands for the Upper Block has grown to three, the two original operands and the $1 \mathrm{ulp.}$

An approach is used which is similar to the 3:2 compression of three operand additions where sum and carry vectors are computed using a single level of gate logic prior to a normal two operand addition. This is performed in the Conversion Combinational Logic Block of Figure 4.8. In this approach only the two original operands for the Upper Block are converted to sum and carry vectors according to the following expressions: 


$$
\begin{aligned}
& s_{n}=A_{n} \oplus B_{n}, 3 \leq n \leq p-1 \\
& c_{n}=A_{n} \cdot B_{n}, 3 \leq n \leq p-1
\end{aligned}
$$

These two vectors then become the operands to the Upper Block summation with the carry vector being offset to the left by one bit as required. The most significant bit of the carry vector can be dropped as it is guaranteed to be insignificant because the data path is padded to cover all possible overflow conditions. The least significant bit of the aligned carry vector will always be zero due to the shift of the carry vector. This bit can be, therefore, set to the conditional pre-carry as determined by the Lower Block operands. The ability to insert the pre-carry condition into the operands after they have been converted is why the condition was not included in the conversion equations. This operation is analogous to compressing the two original operands along with the pre-carry (the third operand) using the normal 3:2 compression method but it has the advantage of eliminating spurious transitions in the operands and reducing the power dissipation. The spurious transitions are caused by the late arrival of the pre-carry signal with respect to the other operands at the Conversion Combinational Logic. The two resultant operands for the Upper Block addition are shown in Figure 4.9 assuming an original operand length of $p$ bits and the precarry condition is $C_{i n 3}$.

The sum and carry vectors for the Upper Block are then summed with a carry in based on the rounding decision as in rounding Method One. The Lower Block summation is performed in the same manner as in Method One. In the case where no pre-carry is per- 
formed then the actions taken with respect to the setting of the LSB of the result and the selection of the upper bits is the same as before. In the case where a pre-carry is performed, it is guaranteed that an additional carry-in will be required due to the rounding decision. The injection of a carry-in to the summation combined with the precarry results in the total carry-in of two as required. The case where a precarry is generated, and no additional carry-in from rounding is required, can not occur as the conditions that lead to a precarry also demand a carry-in from the rounding decision.

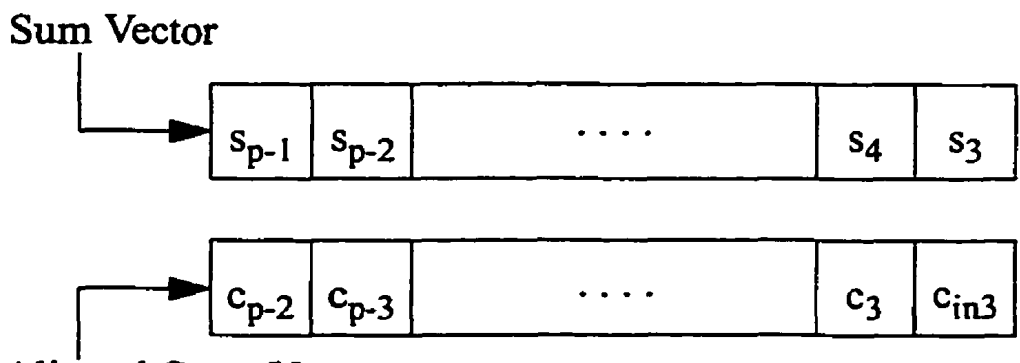

Aligned Carry Vector

FIGURE 4.9. Post Compression Operands - Method Two

\subsection{Internal DataPath Widths}

Using the two methods of CORDIC rounding presented earlier, the errors generated are reduced to $1 / 2$ ulp per iteration, irrespective of the type. The sum of all rounding errors is the number of iterations multiplied by $1 / 2$. This error takes a maximum of $\left\lceil\log _{2}(n / 2)\right\rceil$ bits to represent in binary form, where $n$ is the number of iterations, and so it is guaranteed that the error can not propagate more than $w=\lceil\log (n / 2)\rceil$ bits towards the most significant bit. If the input mantissas are padded by $w$ bits to the right of the LSB then the errors will be contained within the extra bits and will not affect the $b$ bits of desired accuracy. After all the iteration computations are complete, the $w$ padding bits can 
be truncated from the result, having served their purpose, with no loss of accuracy. The addition of the padding bits does not necessitate an increase in the number of rotations required as they are on the least significant end of the mantissas. Iterations involving shifts greater than the bitwidth $b$ would only serve to modify the least significant $w$ bits. These bits are truncated to form the final result and so these extra iterations would not affect the final result.

The conversion of the input data from floating point to block floating point notation requires that care be taken to prevent overflow. An unaccounted growth in magnitude of the mantissas would cause overflow and result in the loss of results despite the fact that the overflow would not have occurred if the computation had been done in floating point form. The upper limit of the mantissa magnitude is 2 and the largest angle of rotation possible in either rotation or vectoring mode of operation is $\pi$. The worst case growth possible using the equations governing rotation in cartesian coordinates occurs when $x$ and $y$ are their maximums and $\theta=0.785$ radians. The growth under these conditions is by a factor of approximately 1.4. One additional bit to the left of the MSB will ensure that the worst possible rotational growth does not result in overflow. Growth also occurs in the iterations due to the scaling factor. In the double shift version of the CORDIC algorithm the scaling factor is steered to a value of 2 . The scaling growth results in one additional bit being required to the left of the MSB bringing the total of additional bits to two. Unlike the bits added to capture the rounding errors, the bits added to capture growth require more iterations to be performed. This is because the least significant bit of the original $b$ bit number is now the least significant bit of $b+2$ bit number due to the two extra bits added to the 
most significant part. The determination of the value of this bit requires a shift of $b+2$, two more than if no growth bits were required. These additional iterations require a re-evaluation of the required number of trailing error bits based on the new number of $b+2$ iterations.

\subsection{Iteration Parameters}

The modified CORDIC iteration equations of the form:

$$
\begin{aligned}
& x_{i+1}=x_{i}-\xi_{i}\left(2^{-S(i)}+\eta(i) 2^{-S^{\prime}(i)}\right) y_{i} \\
& y_{i+1}=y_{i}+\xi_{i}\left(2^{-S(i)}+\eta(i) 2^{-S^{\prime}(i)}\right) x_{i}
\end{aligned}
$$

require that the parameters $S(i), S^{\prime}(i)$ and $\eta(i)$ be specified for the particular implementation. These parameters are potentially different for each desired implementation as they are designed to steer the scaling factor by the end of all iterations and, therefore, depend on the desired accuracy, the bit length of the input data, the number of iterations, etc. These parameters have been provided for one implementation with a bit length and accuracy of 16 bits [17]. Their implementation used both hyperbolic and circular coordinate systems in a programmable PE and so the optimal set of parameters was a single set of parameters satisfying both the hyperbolic and circular systems. Therefore, their parameter set is not necessarily optimal for the proposed generic CORDIC implementation which uses only the circular system. The iterations at the beginning of the algorithm, which perform a double shift, take approximately twice as long as the single shift iterations that appear later on. An optimal solution for this work is one in which the number of double shift iterations is minimized while still retaining the desired level of accuracy. 
For the generic model, the parameters for the various implementations have no requirement to be the same or even similar as the CORDIC PE produced is optimized for a single set of design requirements. For each desired form of the PE, the proper parameters can be used, and the structure of the hardware with respect to the number of double and single rotation iterations can be modified, to produce an optimal result.

The methodology that was followed to generate an optimal set of parameters for the various implementations was based on an iterative process that would produce all sets of parameters for the double shift iterations which satisfied the accuracy requirement [19]. Recall that the effect of the single rotation iterations on the scaling factor is a constant for a particular implementation. The effect of the double rotation iterations was, therefore, required to steer the scaling factor to a value where the remaining single shift iterations would produce the desired final scaling factor of a single power of radix 2, of the form $1 / K=2^{-S}$, where $S$ is an positive integer value, within the allowable error. A program has been written in $\mathrm{C}++$ using nested loops to perform the iterative process required to determine the parameter sets which produce the desired final scaling factor. Inputs to the program were: the number of double shift iterations to be performed, the final shift value required based on the desired accuracy and the maximum value permitted for the $S(i)$ and $S^{\prime}(i)$ parameters of the double rotations. These input values were required to put a reasonable limit on the parameter sets that would be evaluated. The program calculated the scaling factor for all sets of parameters allowed by the inputs and tested them for accuracy against the target value. An example set of the program inputs is: a required final shift value of $18\left(\alpha_{n-1}=\operatorname{atan}\left(2^{-18}\right)\right.$ for 16 bits of accuracy, five double rotations in the range 
$0 \leq S(i) \leq 3$, a maximum $S^{\prime}(i)$ of 16 and $S(i)=4$ for the first single shift iteration. This program was run multiple times for each level of accuracy required. Each run used progressively more double shift iterations, adjusted the range of allowable $S(i)$ and $S^{\prime}(i)$ for the double rotations and adjusted the $S(i)$ of the first single shift iteration.

Sets of parameters that met the basic requirement of accuracy were written to an output file which was later analyzed. The analysis of this file was conducted to identify those parameter sets that also met the conditions of convergence for the rotation angles as shown in equation (4.8). The first condition is that the angle set satisfies the zone of convergence requirement for the desired input angle range which, in this case, is $\theta_{\mathrm{MAX}}=+/-\pi$, The second condition is that the angle set converges to zero with a maximum allowable error of $\alpha_{n-1}$, which is the smallest desired angle resolution. It was found that the total rotation produced by the iterations was not enough to meet the zone of convergence requirement and so, in addition to the iteration rotations, an initial rotation of $\pi / 2$ radians is performed. This rotation has no effect on the scaling factor as $\cos (\pi / 2)=0$.

$$
\begin{gathered}
|\theta| \leq \sum_{j=1}^{n} \alpha_{j}+\alpha_{n-1}=\theta_{M A X} \\
\alpha_{i} \leq \sum_{j=i+1}^{n} \alpha_{j}+\alpha_{n-1}
\end{gathered}
$$

A solution set was then chosen to meet the requirements of each implementation. These results are presented in Table 4.5, Table 4.6 and Table 4.7 for the different accuracy requirements. It is worth noting that the solutions presented are optimal in the sense that they minimize the number of double shift iterations, but they are not unique. There may 
exist, and in most cases do, other shift sequences that satisfy the requirements in the same number of double shift iterations. Each table shows $\theta_{\mathrm{MAX}}$ and the resulting scaling factor in both absolute value format and relative error format. This is to indicate that the scaling factor is within the maximum allowable error. The index values exceed the value of the maximum required shift as some shift values are repeated. The difference between the maximum index value and the maximum shift value is then the number of extra iterations required to steer the scaling factor, or in other words, the cost of steering the scaling factor.

In all of the implementations in this work, the cost is two iterations. The mathematical $\theta_{\text {MAX }}$ for the 24 bit precision parameter set is $1.330 \pi$, but this range is not supported by the proposed implementation. A maximum angle of $1.27 \pi$ is allowed to ensure that there will not be an overflow of the fixed point angle representation which can not represent numbers $\geq 4$.

\begin{tabular}{|c|c|c|c|c|c|c|c|c|c|}
\hline Index i & $\boldsymbol{S}(\boldsymbol{i})$ & $s^{\prime}(i)$ & $n(i)$ & $\alpha(i)$ & Index $\mathbf{i}$ & $S(i)$ & $S^{\prime}(i)$ & $n(i)$ & $\alpha(i)$ \\
\hline 1 & 0 & 4 & 1 & .815692 & 9 & 7 & & & .007812 \\
\hline 2 & 1 & 3 & 1 & .558599 & 10 & 8 & & & .003906 \\
\hline 3 & 1 & 9 & -1 & .462084 & 11 & 9 & & & .001953 \\
\hline 4 & 2 & 8 & -1 & .241299 & 12 & 10 & & & .000977 \\
\hline 5 & 3 & & & .124355 & 13 & 11 & & & .000488 \\
\hline 6 & 4 & & & .062419 & 14 & 12 & & & .000244 \\
\hline 7 & 5 & & & .031240 & 15 & 13 & & & .000122 \\
\hline 8 & 6 & & & .015624 & 16 & 14 & & & .000061 \\
\hline
\end{tabular}

TABLE 4.5. Shift Parameters - 12 bit Accuracy 


\begin{tabular}{|c|c|c|c|c|c|c|c|c|c|}
\hline Index i & $S(i)$ & $S^{\prime}(i)$ & $n(i)$ & $\alpha(i)$ & Index $\mathrm{i}$ & $\boldsymbol{S}(i)$ & $S^{\prime}(i)$ & $n(i)$ & $\alpha(i)$ \\
\hline 1 & 0 & 2 & 1 & .896055 & 13 & 11 & & & .000488 \\
\hline 2 & 1 & 4 & 1 & .512389 & 14 & 12 & & & .000244 \\
\hline 3 & 2 & 4 & 1 & .302885 & 15 & 13 & & & .000122 \\
\hline 4 & 2 & 7 & -1 & .237612 & 16 & 14 & & & $6.104 \mathrm{e}-5$ \\
\hline 5 & 3 & 9 & -1 & .122431 & 17 & 15 & & & $3.052 e-5$ \\
\hline 6 & 4 & & & .062419 & 18 & 16 & & & $1.526 \mathrm{e}-5$ \\
\hline 7 & 5 & & & .031240 & 19 & 17 & & & $7.630 e-6$ \\
\hline 8 & 6 & & & .015624 & 20 & 18 & & & $3.815 e-6$ \\
\hline 9 & 7 & & & .007812 & \multicolumn{5}{|c|}{$i=21,22$ for 18 bit accuracy only } \\
\hline 10 & 8 & & & .003906 & 21 & 19 & & & $1.908 e-6$ \\
\hline 11 & 9 & & & .001953 & 22 & 20 & & & $9.538 \mathrm{e}-7$ \\
\hline 12 & 10 & & & .000977 & & & & & \\
\hline
\end{tabular}

TABLE 4.6. Shift Parameters - 16 and 18 bit Accuracy

\begin{tabular}{|c|c|c|c|c|c|c|c|c|c|}
\hline Index $\mathbf{i}$ & $s(i)$ & $S^{\prime}(i)$ & $n(i)$ & $\alpha(i)$ & Index i & $S(i)$ & $S^{\prime}(i)$ & $n(i)$ & $\alpha(i)$ \\
\hline$\overline{1}$ & $\overline{0}$ & $\overline{9}$ & 1 & .78637 & $\overline{16}$ & $\overline{113}$ & & & .000122 \\
\hline 2 & 1 & 13 & -1 & .46355 & 17 & 14 & & & $6.104 e-5$ \\
\hline 3 & 1 & 6 & -1 & .45107 & 18 & 15 & & & $3.052 e-5$ \\
\hline 4 & 1 & 4 & -1 & .41241 & 19 & 16 & & 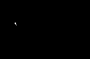 & $1.526 \mathrm{e}-5$ \\
\hline 5 & 2 & 9 & 1 & .24682 & 20 & 17 & & & $7.629 e-6$ \\
\hline 6 & 3 & & & .124355 & 21 & 18 & & & $3.815 e-6$ \\
\hline 7 & 4 & & & .062419 & 22 & 19 & & & $1.907 e-6$ \\
\hline 8 & 5 & & & .031240 & 23 & 20 & & & $9.537 e-7$ \\
\hline 9 & 6 & & & .015624 & 24 & 21 & & & $4.768 e-7$ \\
\hline 10 & 7 & & & .007812 & 25 & 22 & & & $2.384 e-7$ \\
\hline 11 & 8 & & & .003906 & 26 & 23 & & & $1.192 e-7$ \\
\hline 12 & 9 & & & .001953 & 27 & 24 & & & $5.960 \mathrm{e}-8$ \\
\hline 13 & 10 & & & .000977 & 28 & 25 & & & $2.980 \mathrm{e}-8$ \\
\hline 14 & 11 & & & .000488 & 29 & 26 & & & $1.490 \mathrm{e}-8$ \\
\hline 15 & 12 & & & .000244 & & & & & \\
\hline
\end{tabular}

TABLE 4.7. Shift Parameters - 24 bit Accuracy 


\subsection{Accuracy}

The selected algorithm was implemented in MATLAB using a combination of built-in and custom functions. Tests were conducted using randomly generated data with a normal distribution of unit variance and zero mean. The generated numbers were then decomposed into the required mantissa and exponent parts. The mantissas were in normalized form and were converted into 2's complement form of the required length while the exponents were biased by the proper amount. This method of producing data with the desired distribution and then performing the decomposition for the mantissa and exponent is valid for generating properly distributed floating point numbers. It is not valid to generate the mantissas and exponents as separate distributions as, in practice, they are dependent on each other [38]. Mantissas will be generated that essentially cover the entire range of values while the generation of the exponents will be closely grouped around the bias value. The fact that the exponents obtained do not cover their entire possible range is not significant in terms of accuracy for this implementation of the CORDIC algorithm as the exponents are stripped from the mantissas and do not affect the computations at the iteration level. A size of $128 \mathrm{~K}$ samples was selected for each simulation run in order to obtain statistically accurate results.

Simulations were conducted in the vectoring mode using independently generated $x$ and $y$ input values. In vectoring mode the two relevant outputs are the $x$ and $z$ values which correspond to the vector length and angle respectively. The $y$ value is driven to zero during the iterations and is not significant at the output. To determine accuracy, the output values were compared to the results that were obtained using MATLAB's double-preci- 
sion computations and built-in trigonometric functions. The desired value of the vector length $x_{\text {out }}$ is $\sqrt{x^{2}+y^{2}}$, while the desired value for the vector angle $z_{\text {out }}$ is $\operatorname{atan}(y / x)$. The error of the results was determined by taking the absolute value of the difference between the calculated and expected values. The equation for absolute error is:

$$
\text { absolute error }=\mid \text { expected value }- \text { calculated value } \mid=\mathrm{M}_{\mathrm{e}} \times 2^{\mathrm{Ee}}
$$

where $M_{e}$ represents the mantissa value and $E_{e}$ represents the exponent value. Errors in floating point numbers are most commonly quoted in relative terms and, to determine the amount of error in the final result, the error was calculated with respect to the final exponent. Relative error is a more meaningful measure of accuracy than absolute error since the value of the mantissa bits depend on the exponent value. Absolute error gives no indication of whether or not the error is representable within the resolution allowed by the exponent of the result. The relative error was obtained by taking the absolute error and dividing by the result exponent. The equation is:

$$
\text { relative error }=\frac{M_{e} 2^{E_{e}}}{2^{E_{r}}}=M_{e} 2^{E_{e}-E_{r}}
$$

where $E_{r}$ is the value of the result exponent. The relative error is, therefore, the error with respect to the binary point of the result mantissa. To help determine whether or not the error is representable within the resolution of the result, the logarithm of the relative error is obtained:

$$
\log _{2}(\text { relative error })=\log _{2}\left(M_{e} 2^{E_{e}-E_{r}}\right)=\log _{2}\left(M_{e}\right)+E_{e}-E_{r}
$$


The value of the relative error logarithm indicates whether or not the error is representable. Consider the 12 bit mantissa used in data Format 1; there are a total of 12 bits, 10 of which are to the right of the binary point. The value of the least significant bit is, therefore, $2^{-10}$. The logarithm of the smallest representable quantity is then $\log _{2}\left(2^{-10}\right)=-10$. If the value of the relative error logarithm is greater than -10 , it indicates that the error is representable within the result. In this case the result can be considered in error. If, on the other hand, the relative error logarithm is less than -10 it indicates that the error is not representable within the result and, therefore, the result is correct to its limit of precision. This example can be generalized to a $b$-bit mantissa where the smallest resolution is $2^{-b+2}$, and errors are indicated by relative error logarithms greater than $-b+2$. If the result is affected by error then the number of bits which are affected by the error is:

$$
\text { affected bits }=\lceil\log (\text { relative error })-(-b+2)\rceil
$$

The graphs of the error distribution of $x$ and $z$ for the various mantissa widths are shown in Figure 4.10 to Figure 4.17 where the vertical dashed line indicates the boundary between accurate and error affected results. It can be seen by examining the figures that, in general, the results are accurate to the full bitwidth. In a low percentage of cases, the least significant bit of the result is affected. This error is a combination of the angle approximation error, the compensation of the scaling factor error and the rounding errors. For these few sets of input data, the total of all errors is greater than $2^{-b+2}$ despite the fact that individually they are not significant. The percentage of the results where the least significant 
bit was affected is outlined in Table 4.8. The average rate of error for the $x$ and $z$ results was $1.23 \%$ and $0.0 \%$ respectively.

\begin{tabular}{|c|c|c|}
\hline $\begin{array}{c}\text { Mantissa } \\
\text { Width }\end{array}$ & $\begin{array}{c}\text { Output } \\
\text { Value }\end{array}$ & $\begin{array}{c}\text { \% of Samples } \\
\text { Afected }\end{array}$ \\
\hline \multirow{2}{*}{12} & $\mathrm{x}$ & 0 \\
\cline { 2 - 3 } & $\mathrm{z}$ & 0 \\
\hline \multirow{2}{*}{16} & $\mathrm{x}$ & 0.945 \\
\cline { 2 - 3 } & $\mathrm{z}$ & 0 \\
\hline \multirow{2}{*}{18} & $\mathrm{x}$ & 3.94 \\
\hline \multirow{2}{*}{24} & $\mathrm{z}$ & 0 \\
\hline & $\mathrm{x}$ & .045 \\
\cline { 2 - 3 } & $\mathrm{z}$ & 0 \\
\hline
\end{tabular}

TABLE 4.8. Errors in LSB of Result - Vectoring Mode 


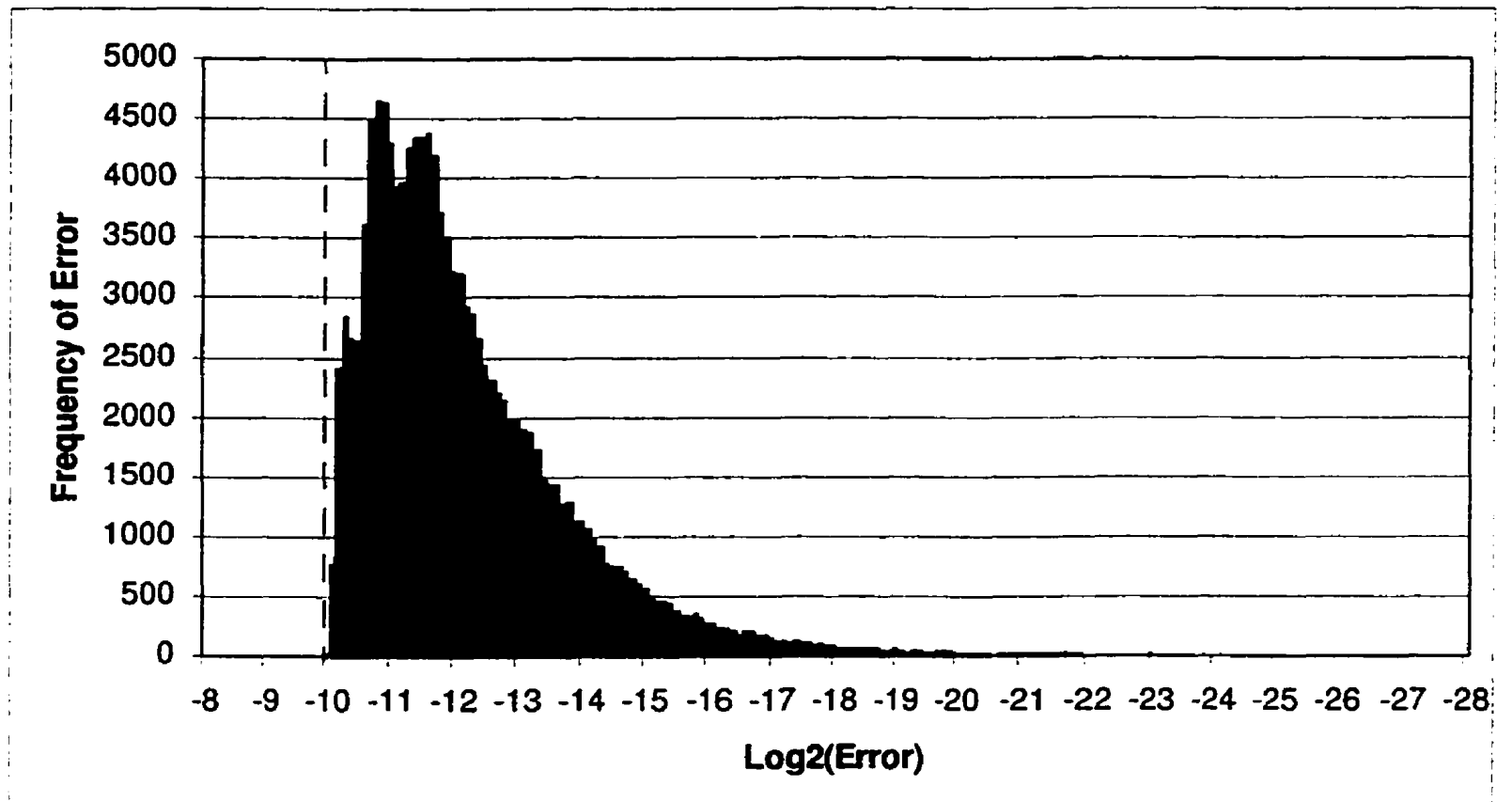

FIGURE 4.10. Error Distribution - X Datapath, 12 Bit Mantissa, Vectoring Mode

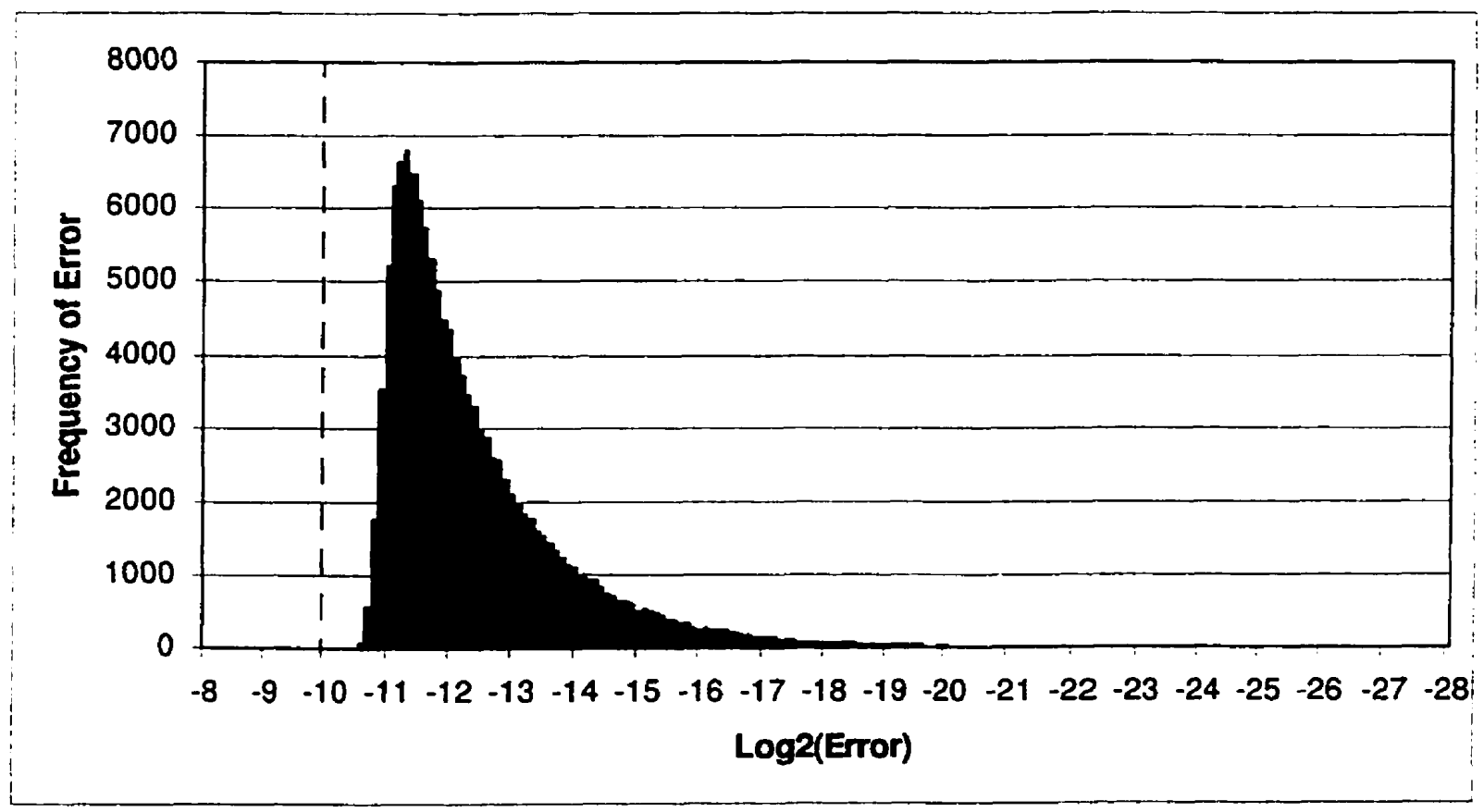

FIGURE 4.11. Error Distribution - Z Datapath, 12 Bit Mantissa, Vectoring Mode 


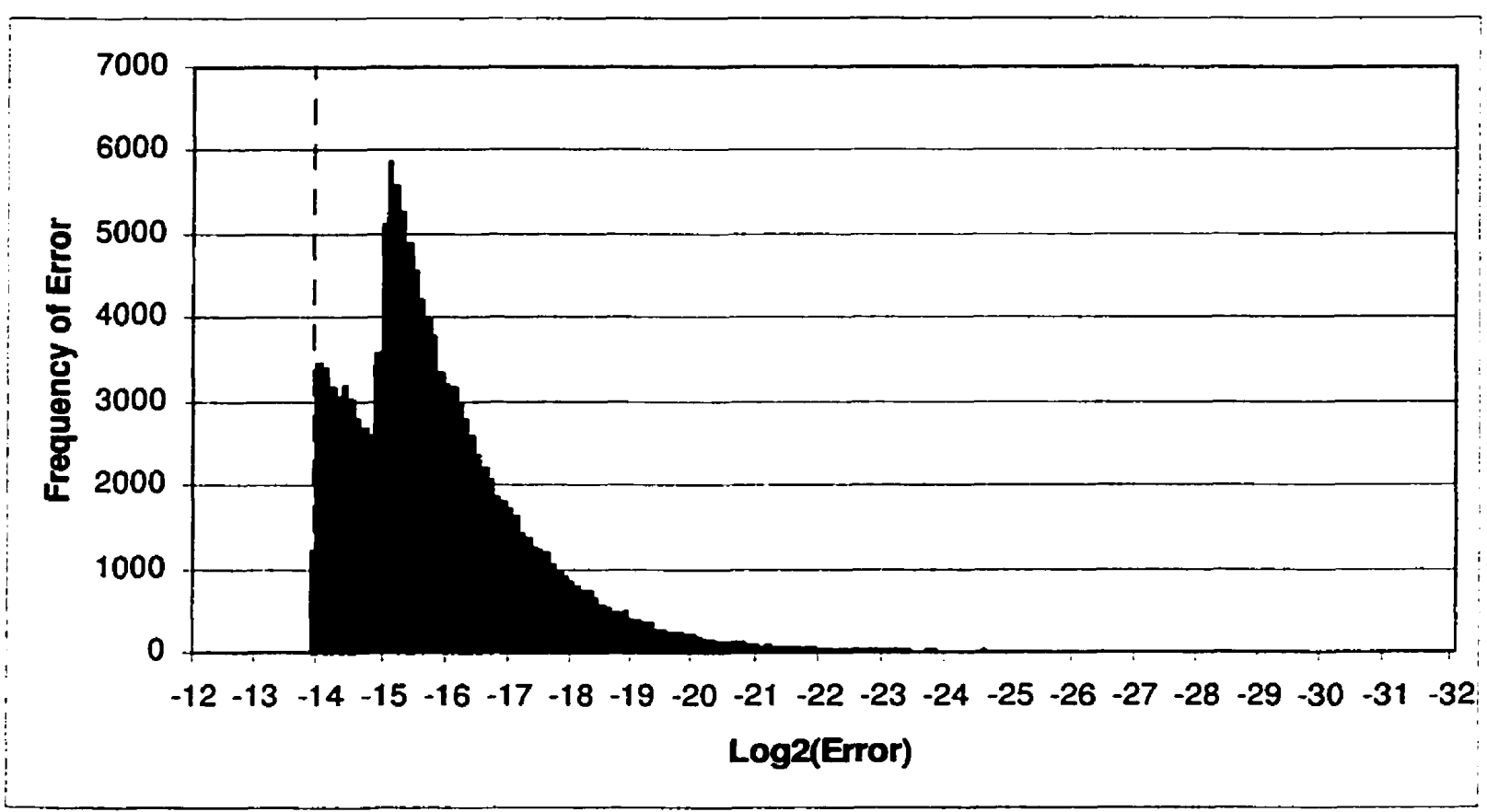

FIGURE 4.12. Error Distribution - X Datapath, 16 Bit Mantissa, Vectoring Mode

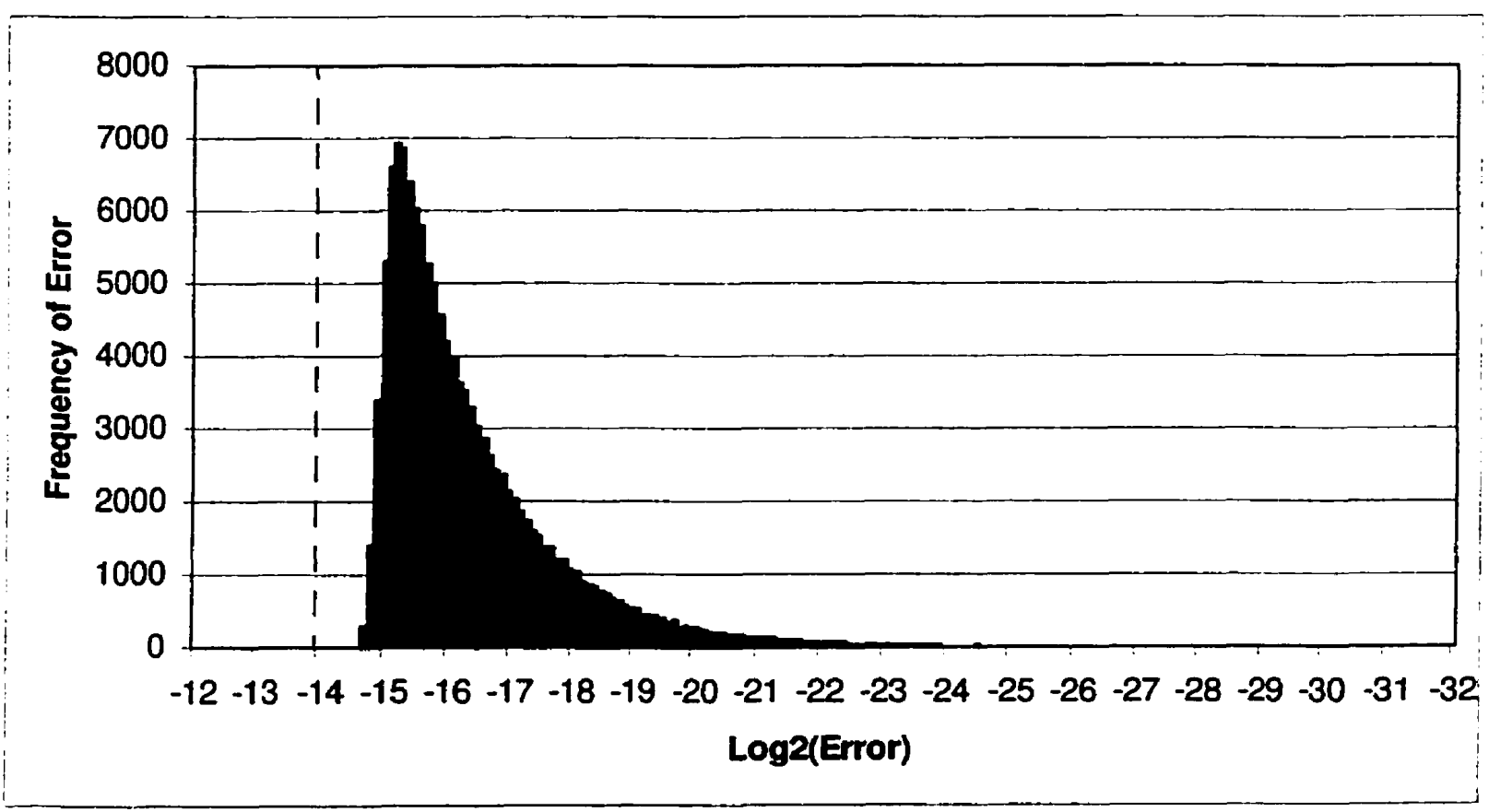

FIGURE 4.13. Error Distribution - Z Datapath, 16 Bit Mantissa, Vectoring Mode 


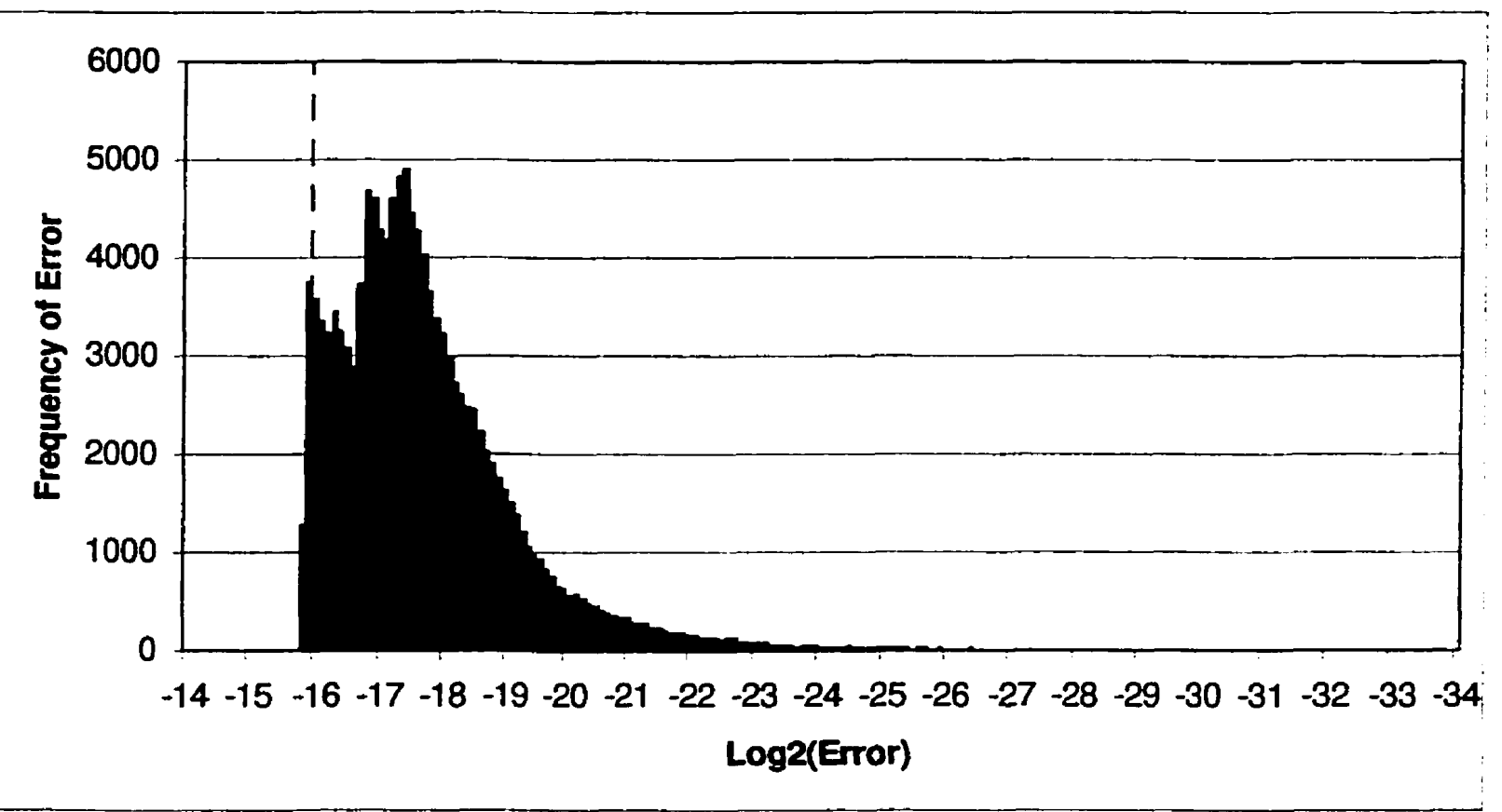

FIGURE 4.14. Error Distribution - X Datapath, 18 Bit Mantissa, Vectoring Mode

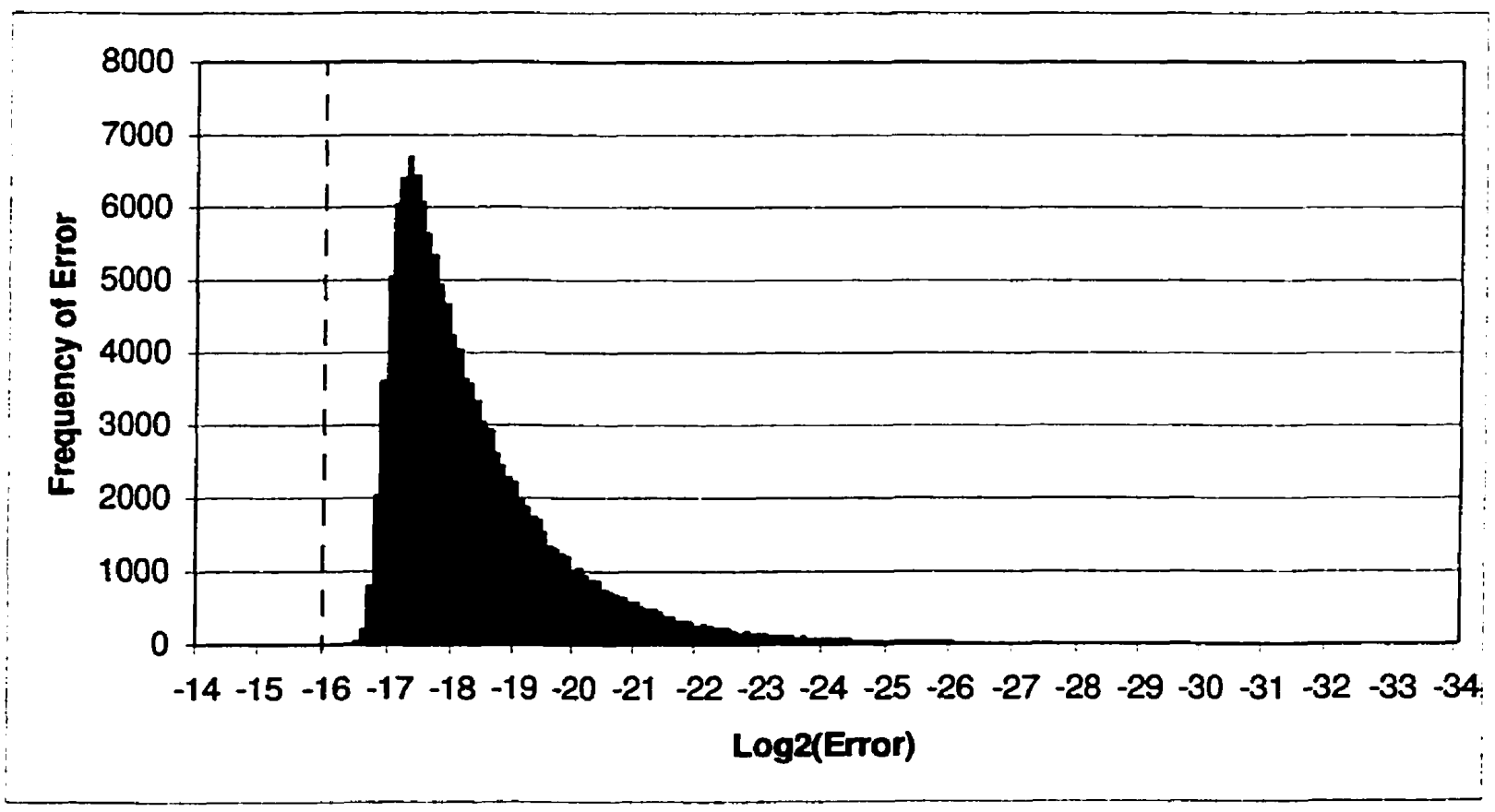

FIGURE 4.15. Error Distribution - Z Datapath, 18 Bit Mantissa, Vectoring Mode 


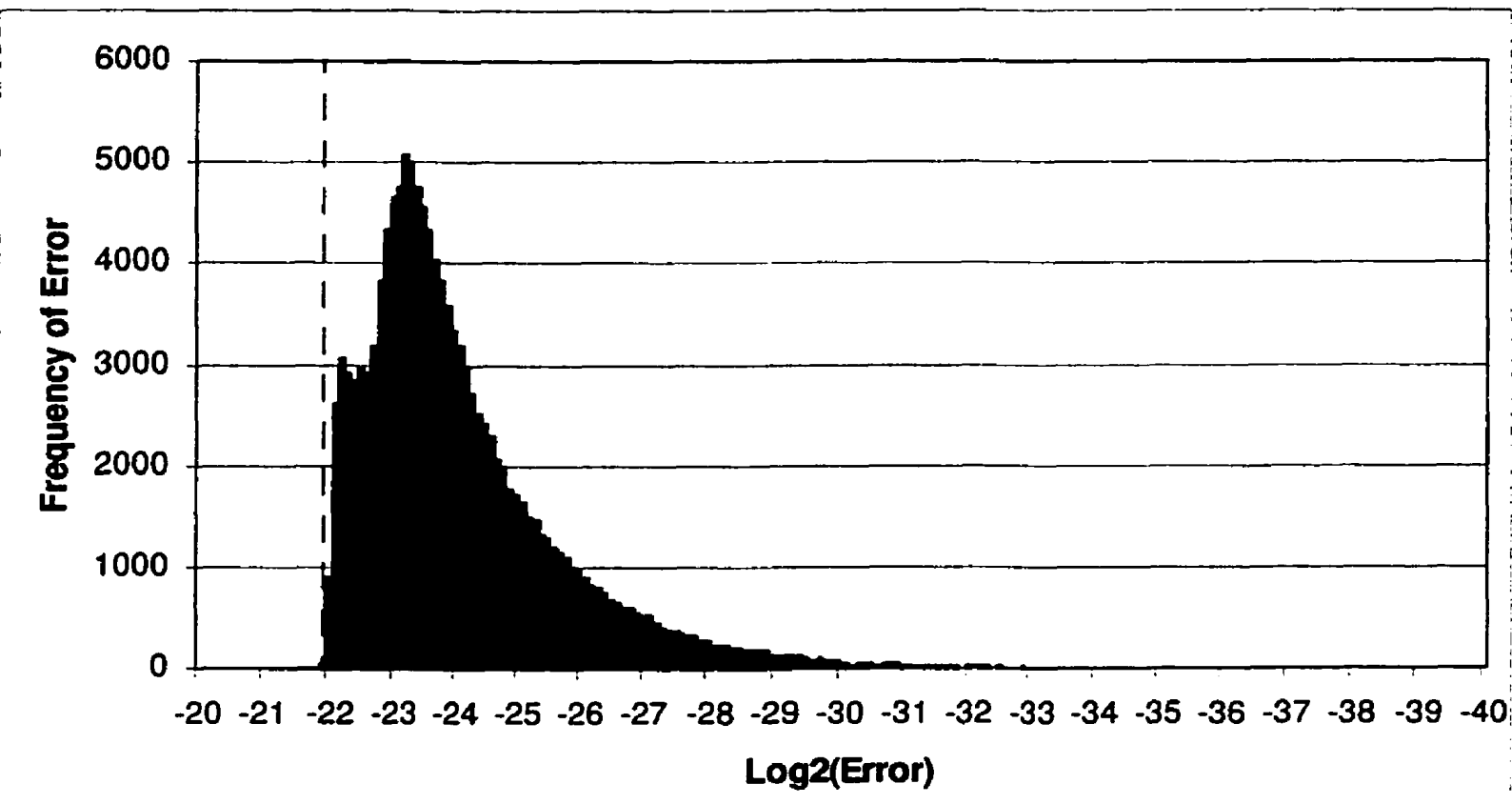

FIGURE 4.16. Error Distribution - X Datapath, 24 Bit Mantissa, Vectoring Mode

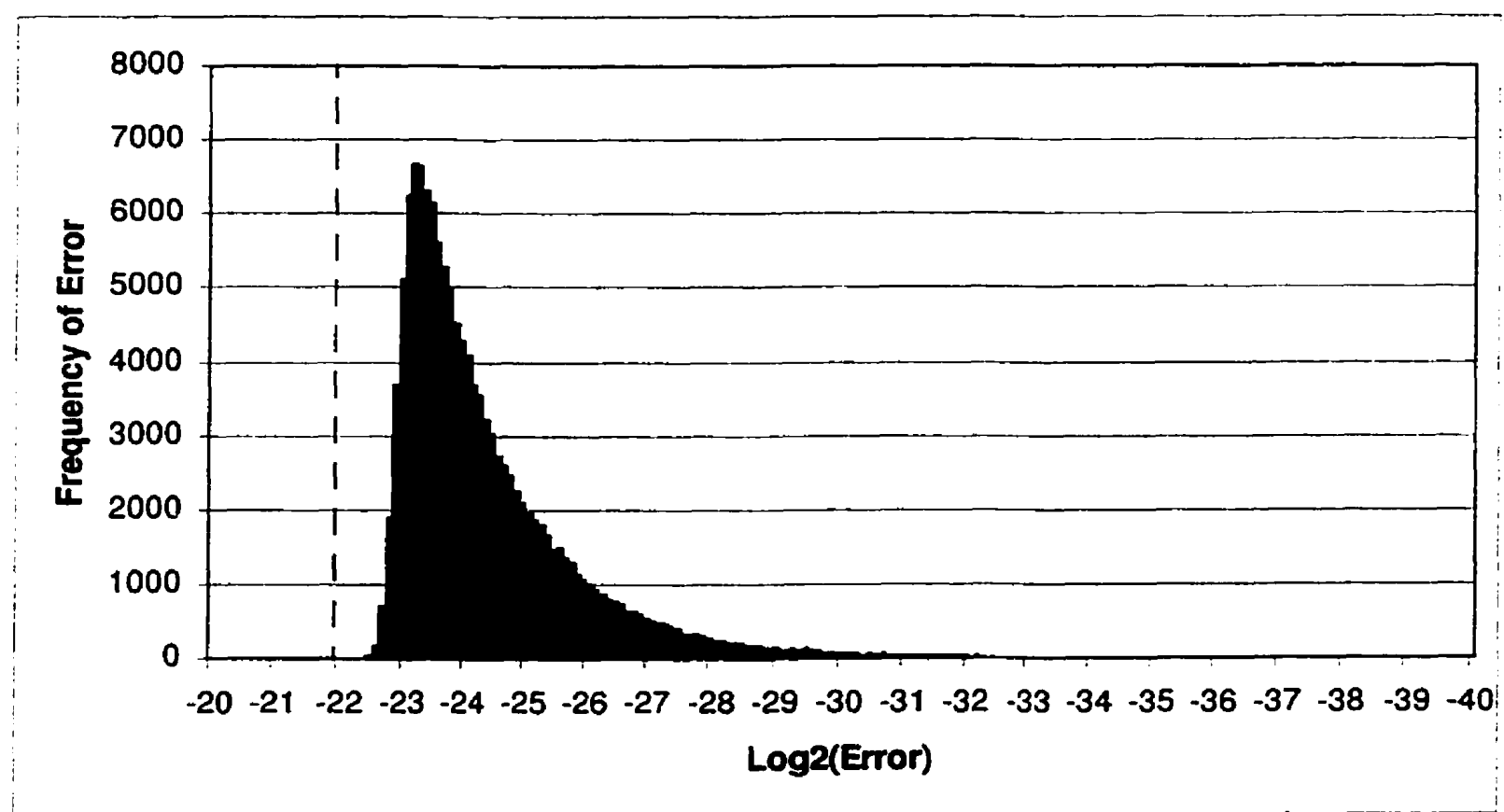

FIGURE 4.17. Error Distribution - Z Datapath, 24 Bit Mantissa, Vectoring Mode 
It is possible to eliminate this error with a combination of increased resolution for the angle representation, an increased number of iterations and an increase in the number of double shift iterations. All of these measures increase area, power consumption and latency for all implementations and only benefit a relatively small percentage of results. It was deemed that the trade-off in performance was too great to justify the small increase in accuracy and so further error reduction measures were not implemented.

Simulations were also conducted in the rotation mode using independently generated $x, y$ and $z$ input values. The $z$ input values were generated by scaling a normal distribution of unit variance and zero mean within a range of $-\pi$ to $+\pi$. In rotation mode the two relevant outputs are the $x$ and $y$ values which correspond to the new cartesian coordinates after rotation. The $z$ value is driven to zero during the iterations and is not significant at the output. Accuracy was again determined using results obtained with MATLAB's doubleprecision computations and built-in trigonometric functions. The desired value of the $x$ and $y$ coordinates is given by:

$$
\begin{aligned}
& x^{\prime}=x \cdot \cos (z)-y \cdot \sin (z) \\
& y^{\prime}=y-\cos (z)+x-\sin (z)
\end{aligned}
$$

The relative error of the results was calculated in the same way as for the vectoring mode of operation according to equation (4.10). The graphs of the error distribution of $x$ and $y$ for the various mantissa widths are shown in Figure 4.18 to Figure 4.25 where the vertical dashed line indicates the boundary between accurate and error affected results.

The results are similar to those obtained in the vectoring mode in that, generally, the results are accurate to the full bitwidth. In a low percentage of cases the least signifi- 
cant bit of the result was affected by errors in the manner outlined above. The percentage of the results where the LSB was affected is outlined in Table 4.9. The average rate of error for the $x$ and $y$ results was $1.57 \%$ and $1.54 \%$ respectively.

It was again deemed that the trade-off in performance to eliminate the errors was too great to justify the small increase in accuracy and so further error reduction measures were not implemented.

\begin{tabular}{|c|c|c|}
\hline $\begin{array}{c}\text { Mantissa } \\
\text { Width }\end{array}$ & $\begin{array}{c}\text { Output } \\
\text { Value }\end{array}$ & $\begin{array}{c}\text { \% of Samples } \\
\text { Affected }\end{array}$ \\
\hline \multirow{2}{*}{12} & $\mathrm{x}$ & 1.895 \\
\cline { 2 - 3 } & $\mathrm{y}$ & 1.925 \\
\hline \multirow{2}{*}{16} & $\mathrm{x}$ & 1.008 \\
\cline { 2 - 3 } & $\mathrm{y}$ & 0.899 \\
\hline \multirow{2}{*}{18} & $\mathrm{x}$ & 1.956 \\
\cline { 2 - 3 } & $\mathrm{y}$ & 1.954 \\
\hline \multirow{2}{*}{24} & $\mathrm{x}$ & 1.438 \\
\cline { 2 - 3 } & $\mathrm{y}$ & 1.373 \\
\hline
\end{tabular}

TABLE 4.9. Errors in LSB of Result - Rotation Mode 


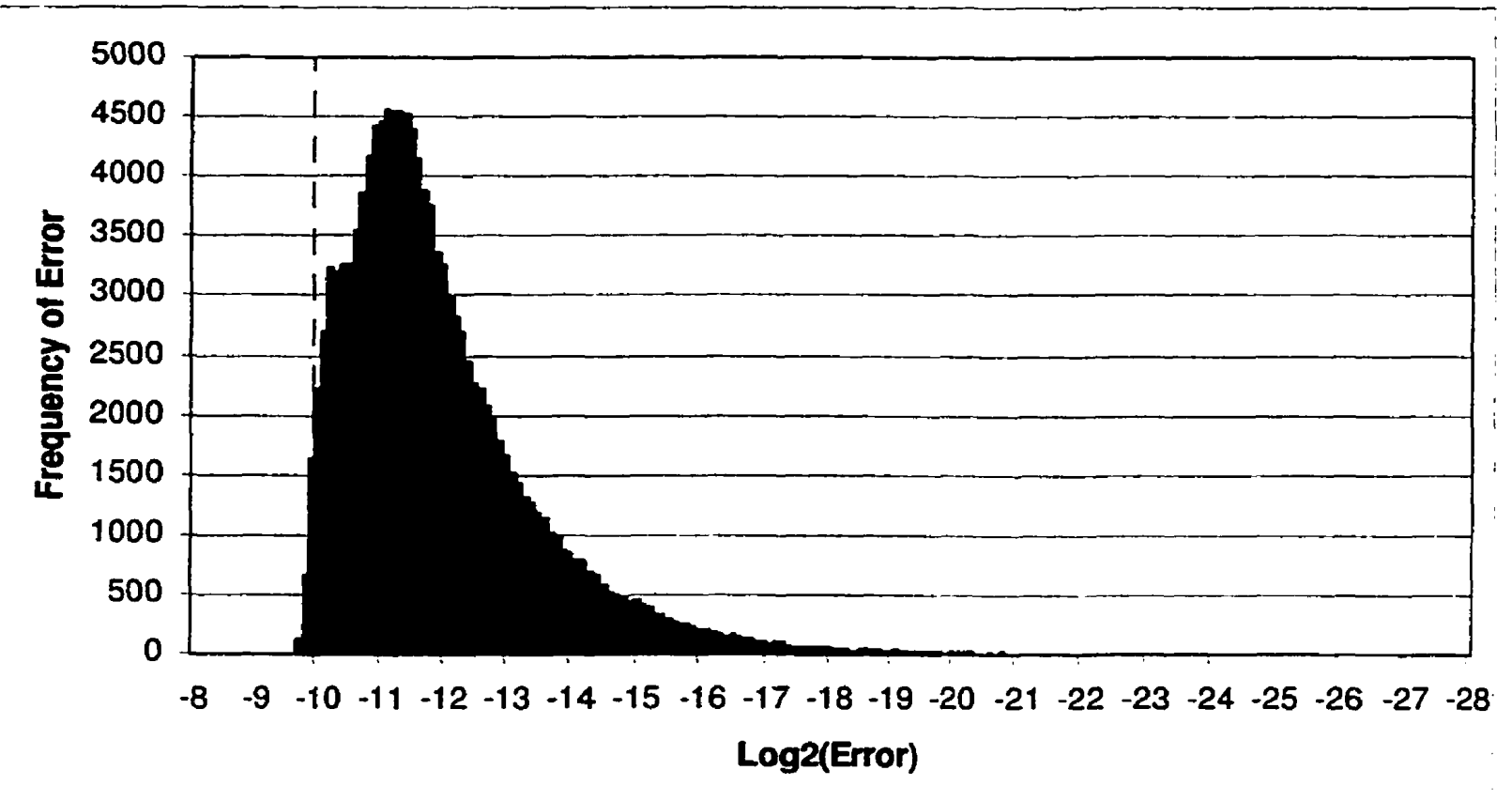

FIGURE 4.18. Error Distribution - X Datapath, 12 Bit Mantissa, Rotation Mode

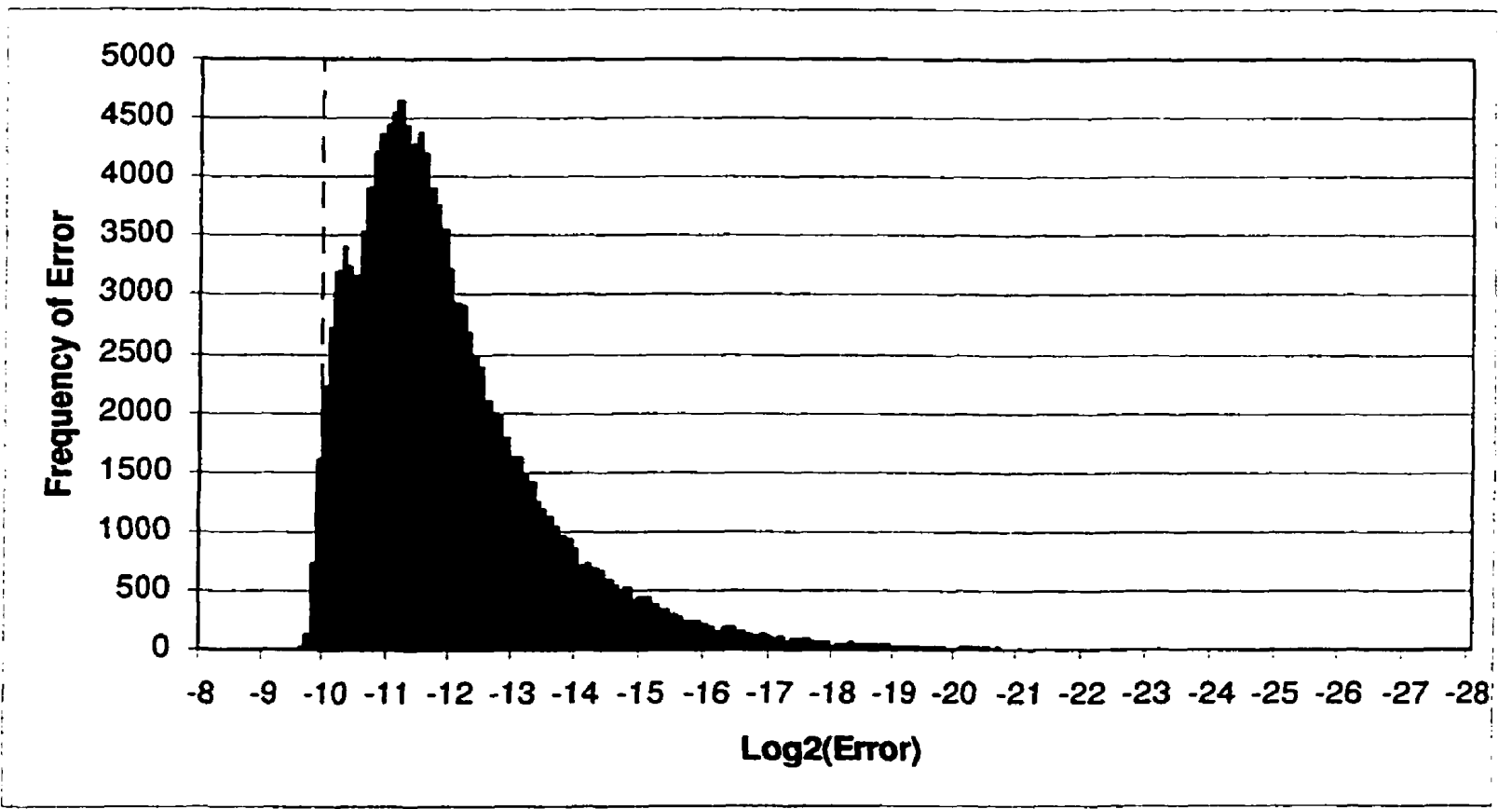

FIGURE 4.19. Error Distribution - Y Datapath, 12 Bit Mantissa, Rotation Mode 


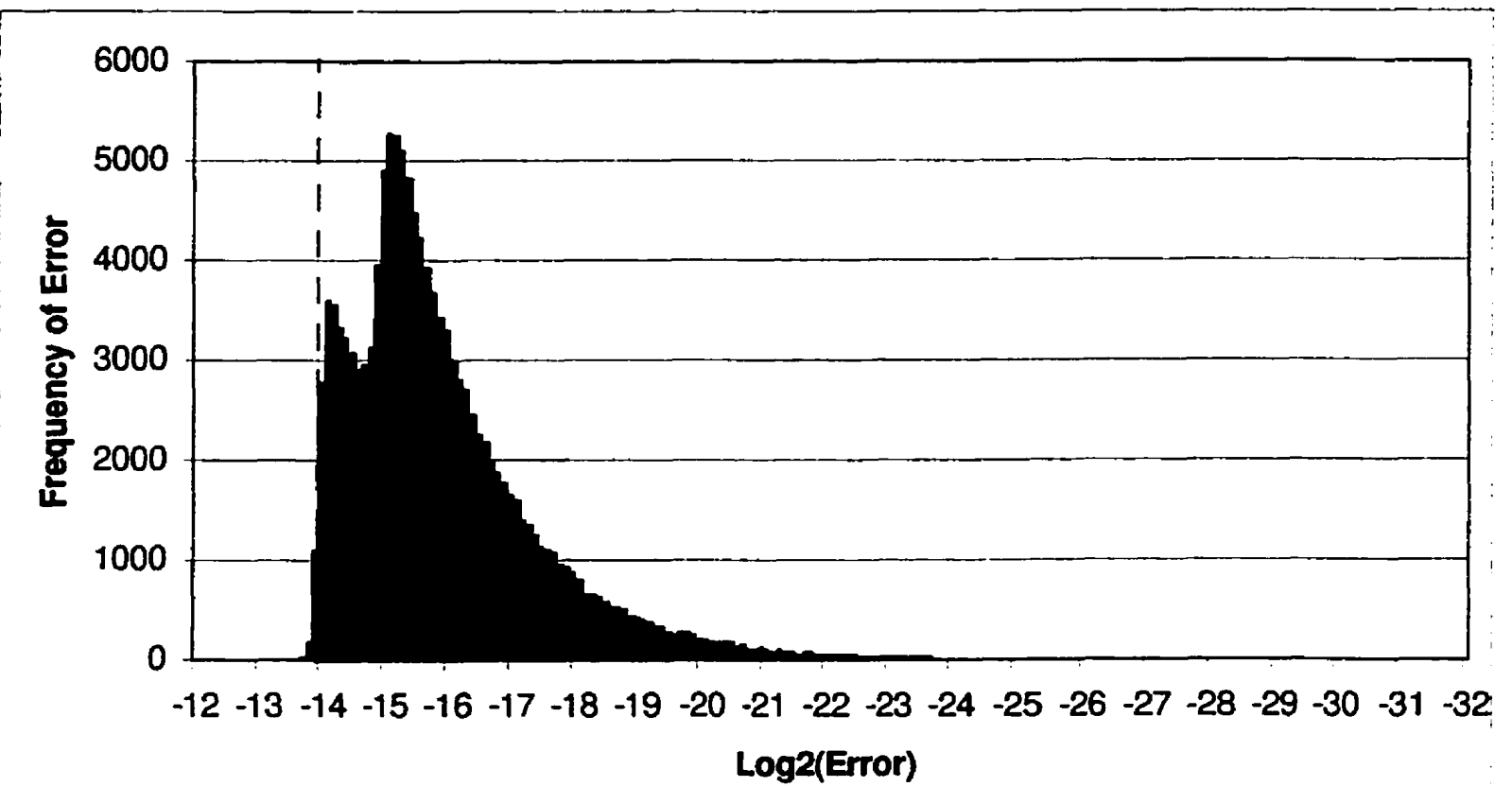

FIGURE 4.20. Error Distribution - X Datapath, 16 Bit Mantissa, Rotation Mode

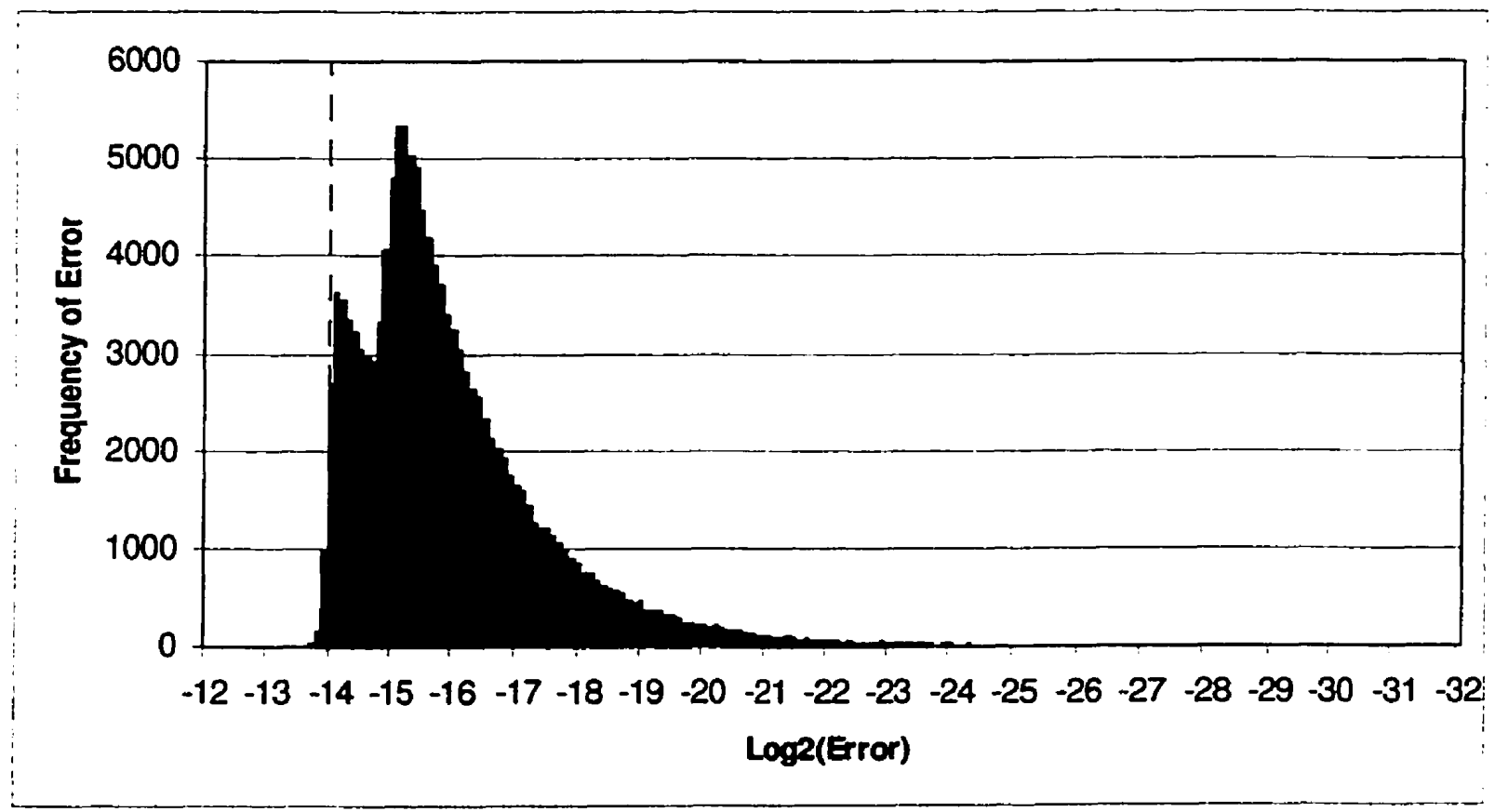

FIGURE 4.21. Error Distribution - Y Datapath, 16 Bit Mantissa, Rotation Mode 


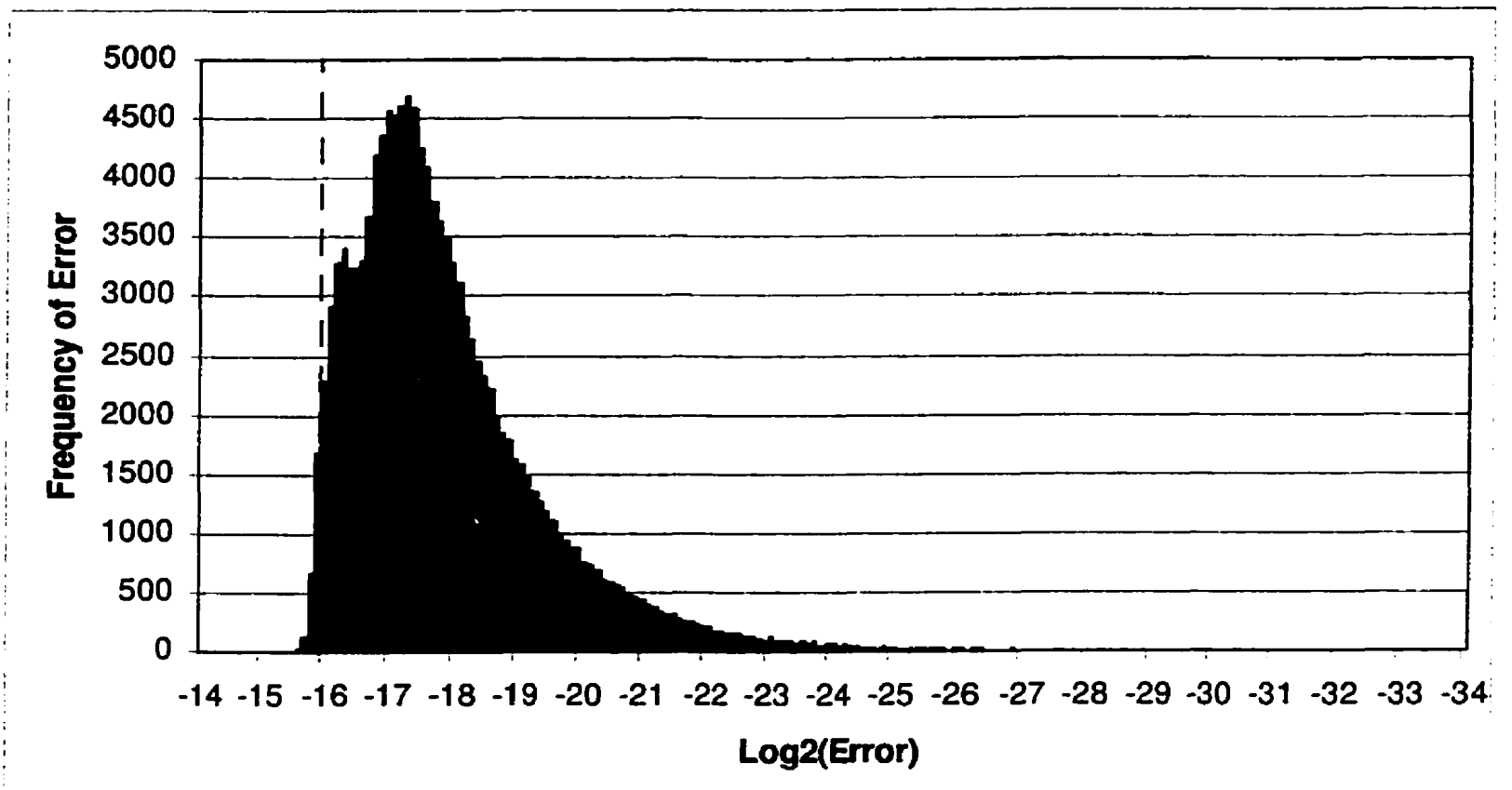

FIGURE 4.22. Error Distribution - X Datapath, 18 Bit Mantissa, Rotation Mode

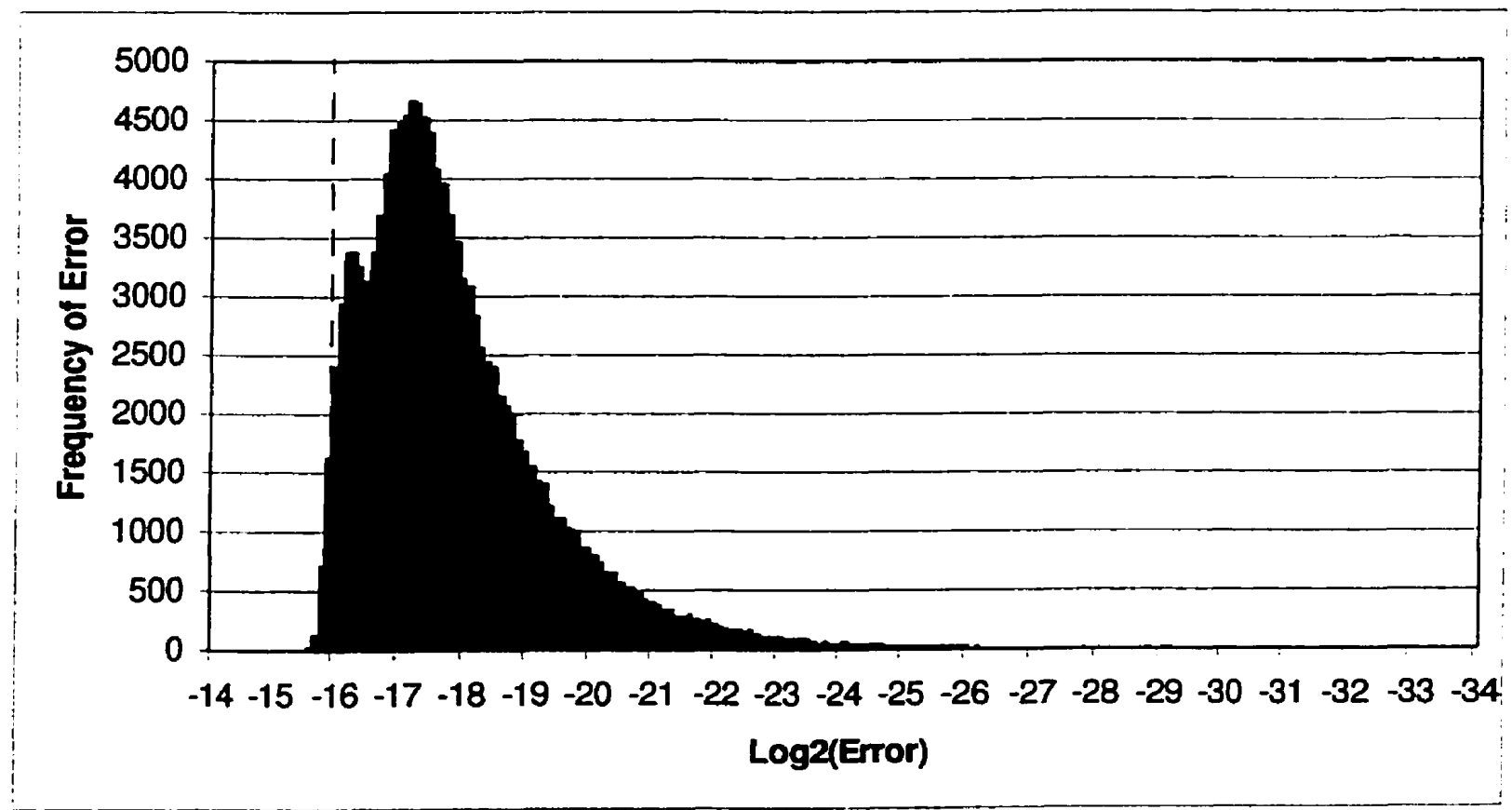

FIGURE 4.23. Error Distribution - Y Datapath, 18 Bit Mantissa, Rotation Mode 


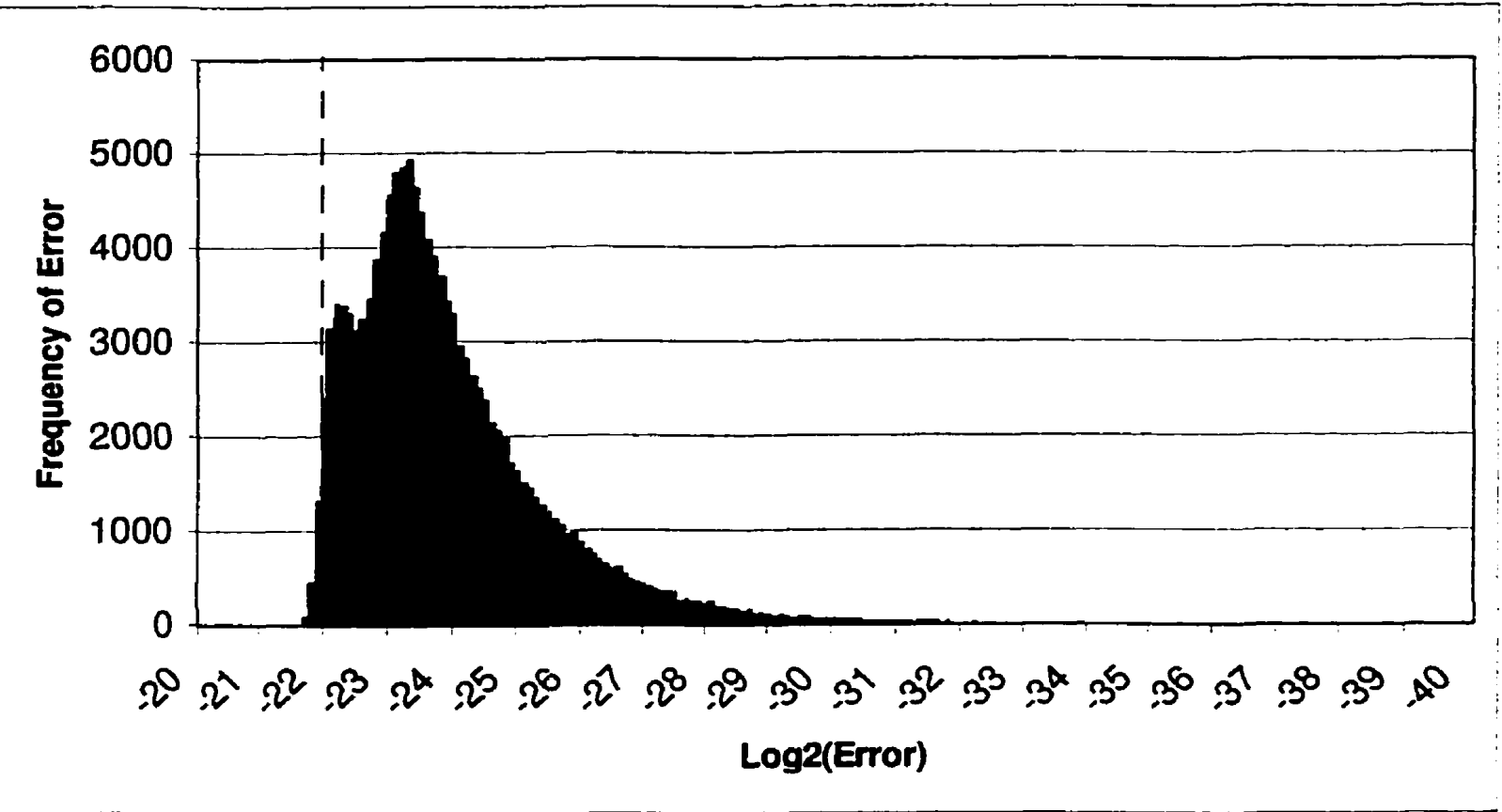

FIGURE 4.24. Error Distribution - X Datapath, 24 Bit Mantissa, Rotation Mode

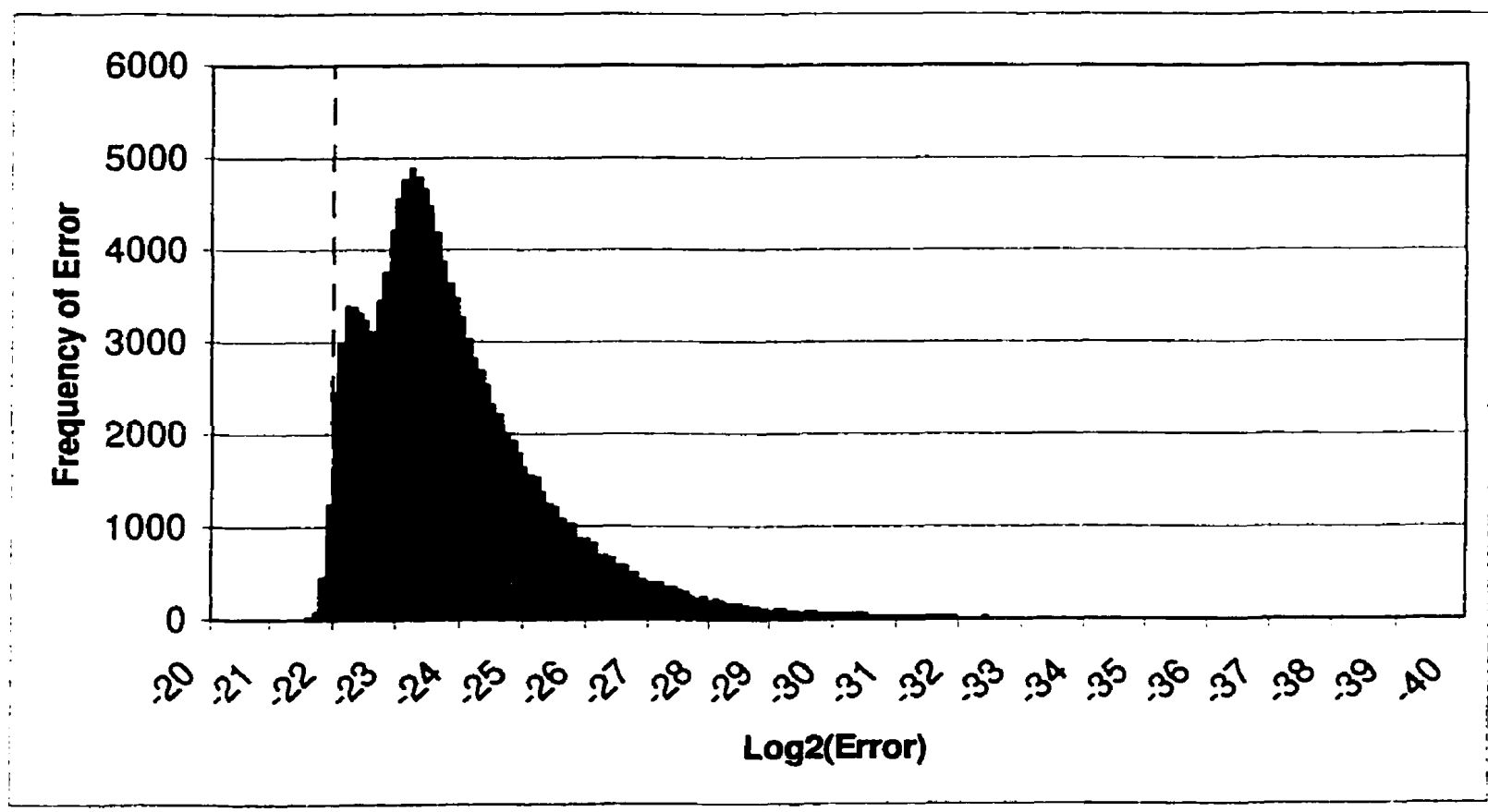

FIGURE 4.25. Error Distribution - Y Datapath, 24 Bit Mantissa, Rotation Mode 


\section{CHAPTER 5}

\section{CORDIC PE ARCHITECTURE}

The CORDIC processing element is composed of three main blocks. The first block is the preprocessor which conditions the incoming data for block floating point operations, determines a working exponent and also performs the first rotation of $\pi / 2$ radi-

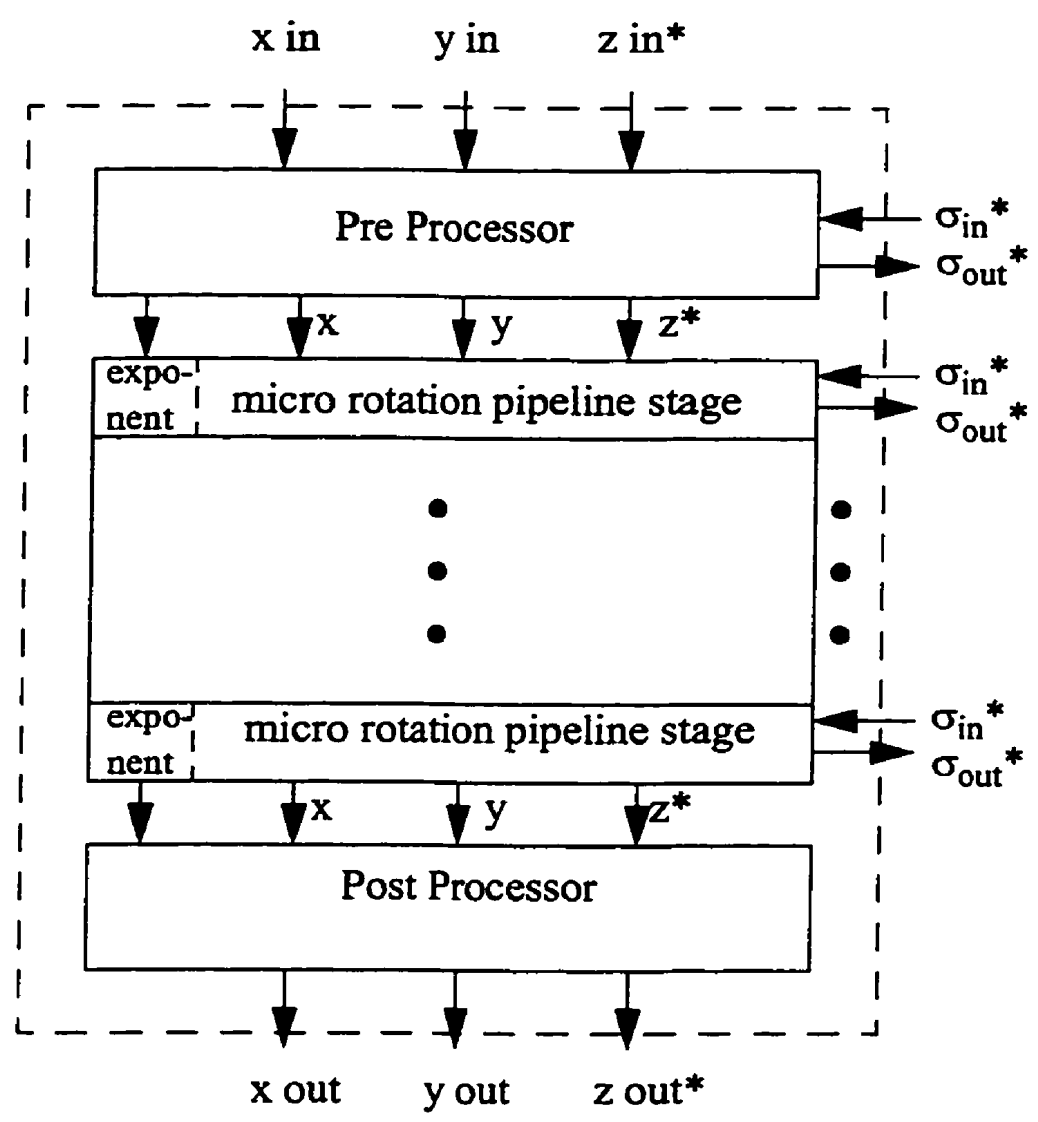

FIGURE 5.1. Floating Point CORDIC Architecture 
ans. The second block is composed of the pipeline stages which perform the remainder of the required rotations according to the double shift CORDIC algorithm. The third block is the post processor which performs the compensation for the scaling factor, applies the working exponent to the resultant mantissas, determines the final mantissa values and adjusts the exponents if required. The post processor also performs normalization if desired. These functional blocks are shown in Figure 5.1. The input/output signals and datapaths marked with an asterix are those that are implementation dependent and are not present in all versions of the CORDIC PE. The architecture of each of the blocks will be described in detail in the following sections.

\subsection{Preprocessor}

The preprocessor block can be broken down into two functional sub-blocks. The first sub-block performs the alignment of the $\mathrm{x}$ and $\mathrm{y}$ floating point mantissas and selects the larger input exponent as the working exponent. The second sub-block performs the first rotation of $\pi / 2$ radians in the appropriate direction. As part of this work, two different versions of the alignment sub-block were developed in order to allow for the optional application of advanced power reduction techniques. The architecture of the first version includes activity scaling measures while the architecture of the second does not. The selection of a specific version is dictated by the datapath width and the distribution of the $x$ and $y$ inputs. The two versions of the alignment sub-block and the rotation sub-block will be examined in the following sections. 


\subsubsection{Alignment Sub-Block Version I}

Version I of the Alignment sub-block [39] of the preprocessor performs the mantissa alignment of the $x$ and $y$ input values and the selection of the working exponent using activity scaling to reduce power consumption. Inputs to the sub-block are the $x$ and $y$ mantissas in 2's complement form and the $x$ and $y$ biased exponents. The outputs are the $x$ and $y$ mantissas and the common working exponent. The overall architecture of the sub-block is shown in Figure 5.2.

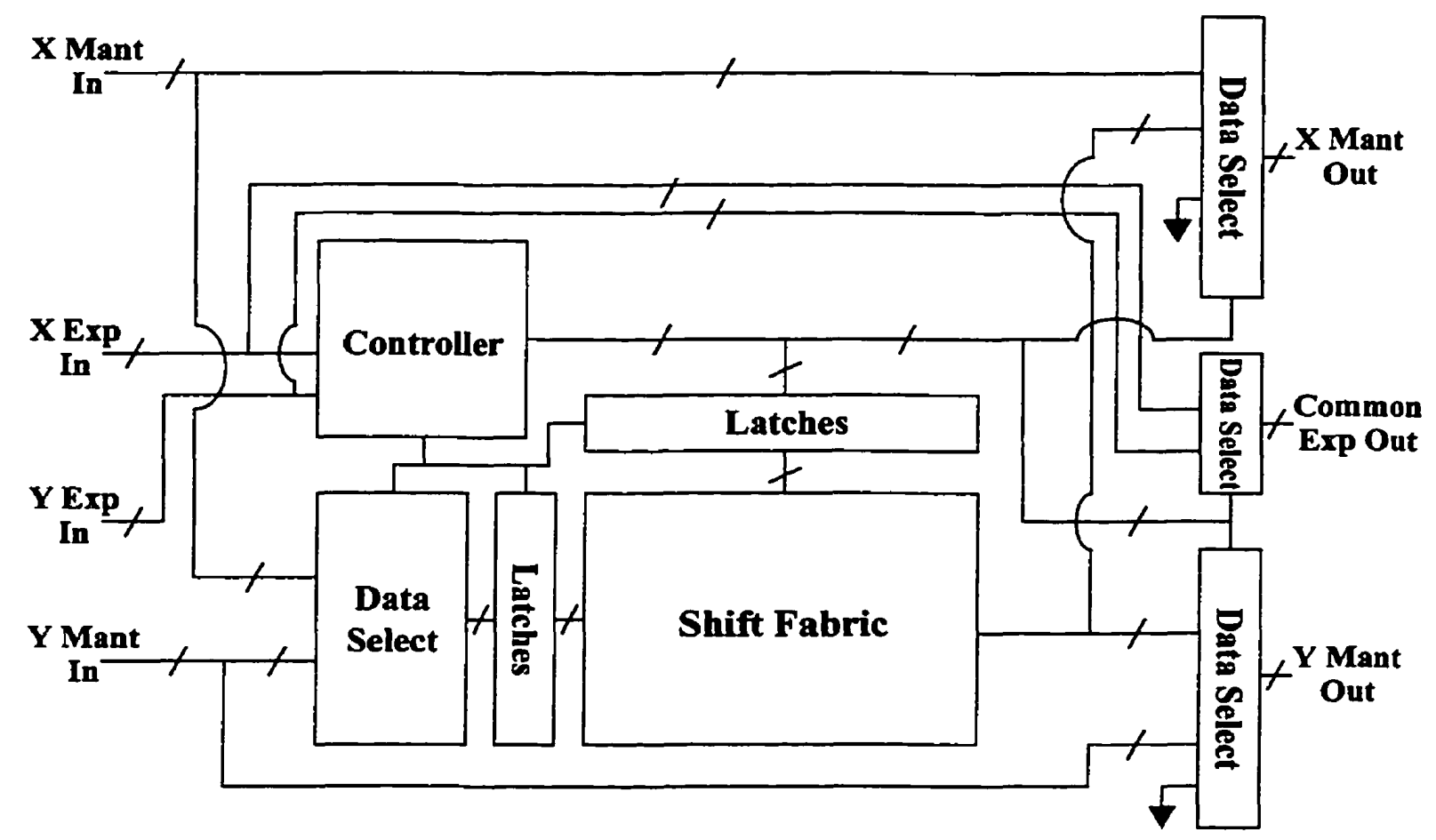

FIGURE 5.2. Preprocessor Alignment Block - Version I

Activity scaling is a method whereby the power consumption of a circuit is reduced by eliminating unnecessary switching on unused parts of the circuit. In the case of the Shift Fabric, which is at the heart of the Alignment sub-block, this means that if it is not required to perform useful shifting then it will not exhibit switching on any of its 
nodes. The first step in achieving this activity scaling is to first determine if the switch fabric is required for the alignment operation. For the alignment of two floating point numbers, a shift is not required for two numbers which have the same input exponent as they are already aligned. It is also true that if the exponents of 2's complement floating point numbers differ by a quantity equal to or exceeding their mantissa width, then the aligned smaller number's mantissa is zero in which case it can be set directly without use of the barrel shifter. In cases where the barrel shifter is not required, it is possible to prevent it from needlessly switching if the previous inputs are maintained. Under these conditions the output of the shift fabric would not have any relevance to the current set of input data, but that is of no consequence as the output of the barrel shifter need not be used to produce the aligned mantissas.

Now that it is apparent that activity scaling is possible, it is vital to determine what actions are required to produce the proper outputs under all conditions. The common exponent must be one of the two input exponents and its value is equal to the larger of the two, or either of the two in the case of equal exponents. To determine the proper output it is, therefore, required to compare the magnitude of the two input exponents. The post alignment output mantissas can take any of three values depending on the input conditions. In the case where the input mantissa, either $x$ or $y$, is a part of the input floating point number with the largest exponent, then the applicable output mantissa is simply the same as the input. This condition also occurs when both input exponents are equal in which case both the $x$ and $y$ output mantissas are unmodified from the inputs. The second possible value for the output mantissas is the zero condition described above where all bits are 
forced to zero. To determine this condition it is necessary to compute the absolute difference in exponents and compare that to the mantissa's bitwidth. In cases where the difference is equal to, or greater than, the bitwidth then the zero condition exists for the mantissa corresponding to the lesser exponent value. The third and final possibility for the output mantissas is that it is a shifted version of the input mantissa. In cases where the difference in exponents is less than the mantissa width it is necessary to shift the mantissa of the lesser exponent by the difference in order to align the two mantissas. This third possibility is the only case where the shift fabric performs any useful function. To properly shift the lesser valued mantissa the absolute difference in exponent must be calculated as well as the operand with the larger exponent. In summary the determination of the largest exponent, the absolute difference in exponents and a comparison of the absolute exponent difference to the mantissa bitwidth are required in order to properly determine the outputs.

One's complement adders are ideal [39] for function implementations of the type $|A-B|$ involving integer data $A$ and $B$. The area/delay/power measures of these units make them attractive for low power/high speed applications. In addition, I's complement adders inherently perform a comparison function which makes them even more attractive for this specific application. For absolute value calculations, the power consumption and silicon area of 1's complement adders are about $50 \%$ of their 2's complement counterparts while meeting the same timing goals. Coupled with no increase in area to perform the comparison functions, the benefits of using l's complement adders for this sub-block are significant. 
In I's complement adders, the conditional carry outputs contain significant information regarding the relative magnitudes of the input operands [39]. The determination of conditions such as $A>B, A \leq B, B>A, B \leq A, A=B$ and $A \neq B$ is relatively straight forward. The significance of the various combinations of conditional carry outputs is shown in Table 5.1 where $C_{\text {out }}(0)$ and $C_{\text {out }}(1)$ represent the conditional carry output from the MSB position of the adder anticipating input carries of 0 and 1 respectively.

\begin{tabular}{|c|c|l|}
\hline Cout $(0)$ & Cout $(1)$ & \multicolumn{1}{|c|}{ Significance } \\
\hline \hline 0 & 0 & $\mathrm{C}_{\text {out }}(1)=0 \Rightarrow \mathrm{A}<\mathrm{B}$ \\
\hline 0 & 1 & $\mathrm{C}_{\text {out }}(0)=0$ and $\mathrm{C}_{\text {out }}(1)=1 \Rightarrow \mathrm{A}=\mathrm{B}$ \\
\hline 1 & 0 & Impossible Condition \\
\hline 1 & 1 & $\mathrm{C}_{\text {out }}(0)=1 \Rightarrow \mathrm{A}>\mathrm{B}$ \\
\hline
\end{tabular}

TABLE 5.1. Significance of Conditional Carry Outputs

The circuit to calculate the conditional carry outputs is shown in Figure 5.3 where the two exponents are $A$ and $B$, and $A_{0}$ represents the L.SB and $A_{7}$ the MSB of exponent $A$. The value of the potential shift, namely $|A-B|$, is calculated in the sum portion of the same 1 's complement adder. The circuit is shown in Figure 5.4 where $A$ and $B$ are again the two exponents and where the conditional carry terms $C(0)$ and $C(1)$ are those extracted from the carry chain presented in Figure 5.3. No additional hardware is necessary to generate the signals for the $A \oplus B$ terms as these have been previously determined as part of the carry chain.

The final sum bits are generated using the condition $A>B$ which is $\mathrm{C}_{\text {out }}(0)$ from the carry chain. A $\mathrm{C}_{\text {out }}(0)=1$ indicates that $A>B$ is true and, therefore, the sum generated 
from the $X O R$ gates and multiplexers already represents a positive number. $A C_{\text {out }}(0)=0$ indicates that $A<=B$ and that the sum generated from the XOR gates and multiplexers represents a negative (or zero) number and must be complemented to obtain the desired positive result. The value of the LSB of the sum, $S_{0}$, is inverted irrespective of the value of the end around carry. If the end around carry is a 0 the result is negative and must be

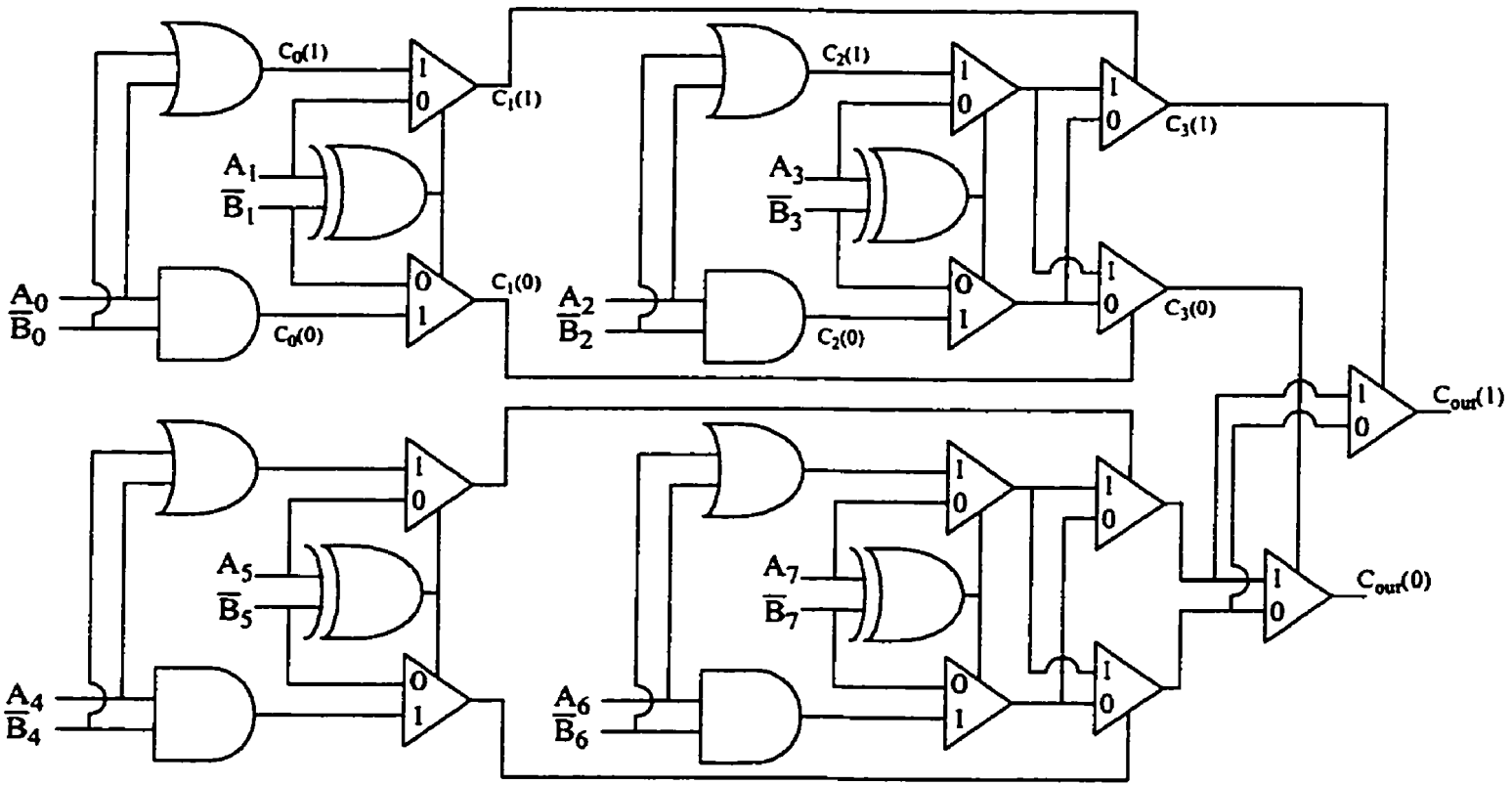

FIGURE 5.3. Evaluation of 'End Around Carry' for $|A-B|$ Computation

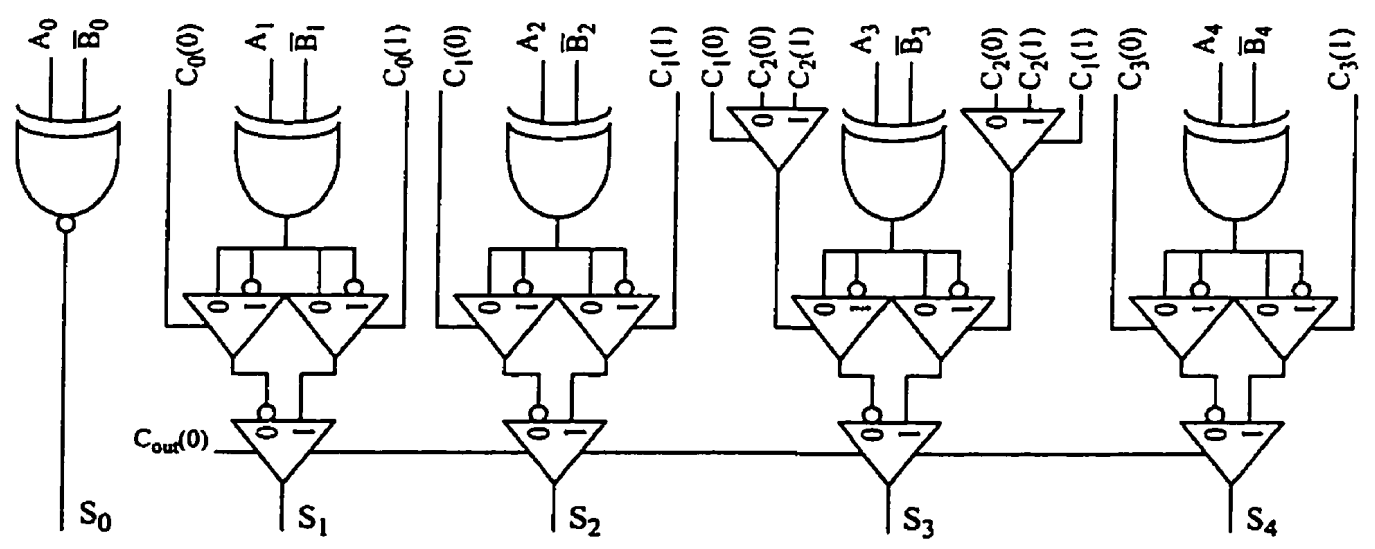

FIGURE 5.4. Generation of Sum Bits for $|A-B|$ Computation 
inverted. When the end around carry is true then a 1 must be added to the LSB position thus resulting in an inversion. The value of the sum bits is required to control the amount of a shift when one is required. If there is a valid shift condition, as explained below, then the shift amount is guaranteed to be less than or equal to the mantissa width. For the range of implementations in this work, it takes a maximum of five bits to represent the greatest possible valid shift value, and so only five bits of the sum are required.

It is now left to determine if the exponent difference $|A-B|$ lies within the range of useful shifts and, therefore, requires the use of the shift fabric to produce the outputs. The condition that is of interest is $A-B>K$ where $K=b-1$. This can be rearranged to the condition $A-B-K>0$, which can be calculated using a 1 's complement carry chain similar to what was done for the exponent comparison. If the carry output is a 1 then the condition is true and the barrel shifter is not required to perform the alignment operation. A carry output of 0 on the other hand does not guarantee that the condition $B-A>K$ holds. For this operation there are three terms which requires compression prior to the actual comparison. The 1 's complement evaluation of $A-B-K>0$ may require the addition of a correction bit of 1 at the LSB position due to the third term. To avoid this, the actual terms used are $A$ ( + ve), $\bar{B}$ (1's complement of $B$ ) and $L=\bar{K}+1$ (2's complement of $K)$. The value $K$ is a known constant for any given CORDIC implementation. This will then allow the computation of sum $(S)$ and carry $(C)$ vectors with at most three levels of logic gates. The three terms can be compressed into sum and carry vectors as given by: 


$$
S_{i}=A_{i} \oplus \bar{B}_{i} \oplus L_{i}, \quad \forall i
$$

and

$$
C_{i}=A_{i} \bar{B}_{i}+L_{i}\left(A_{i}+\bar{B}_{i}\right), \quad \forall i
$$

where $L_{i}$ represents the $i^{\text {th }}$ bit of the 2's complement of $K$. The pre and post compression operands are shown in Figure 5.5 a and b respectively, where $S$ and $C$ represent the sum and carry after compression. The LSB does not affect the calculation as only the carry

\begin{tabular}{|c|c|c|c|c|c|c|c|c|}
\hline 0 & $\mathrm{~A} 7$ & $\mathrm{~A} 6$ & $\mathrm{~A} 5$ & $\mathrm{~A} 4$ & $\mathrm{~A} 3$ & $\mathrm{~A} 2$ & $\mathrm{~A} 1$ & $\mathrm{~A} 0$ \\
\hline 1 & $\overline{\mathrm{B} 7}$ & $\overline{\mathrm{B} 6}$ & $\overline{\mathrm{B} 5}$ & $\overline{\mathrm{B} 4}$ & $\overline{\mathrm{B} 3}$ & $\overline{\mathrm{B} 2}$ & $\overline{\mathrm{B} 1}$ & $\overline{\mathrm{B} 0}$ \\
\hline 1 & $\mathrm{~L} 7$ & $\mathrm{~L} 6$ & $\mathrm{~L} 5$ & $\mathrm{~L} 4$ & $\mathrm{~L} 3$ & $\mathrm{~L} 2$ & $\mathrm{~L} 1$ & $\mathrm{~L} 0$ \\
\hline
\end{tabular}

a) Input data for evaluating $A-B>K$ condition

\begin{tabular}{|c|c|c|c|c|c|c|c|c|}
\hline $\mathrm{C} 7$ & $\mathrm{C} 6$ & $\mathrm{C} 5$ & $\mathrm{C} 4$ & $\mathrm{C} 3$ & $\mathrm{C} 2$ & $\mathrm{C} 1$ & $\mathrm{C} 0$ & \\
\cline { 1 - 8 } & $\mathrm{S} 7$ & $\mathrm{~S} 6$ & $\mathrm{~S} 5$ & $\mathrm{~S} 4$ & $\mathrm{~S} 3$ & $\mathrm{~S} 2$ & $\mathrm{~S} 1$ & \\
\hline
\end{tabular}

b) Input data after bit compression

\section{FIGURE 5.5. Data Presentation for Evaluating A - B > K Condition}

chain is being evaluated. In fact the final sum is not required to test the condition $A-B-K>0$, and is therefore never computed. The logic diagram for the condition evaluation is shown in Figure 5.6. To determine if the condition $B-A>K$ is true without waiting for the determination of $A>B$, which would delay the generation of the control signal, the hardware as discussed for $A-B>K$ is duplicated to allow for concurrent computation. 


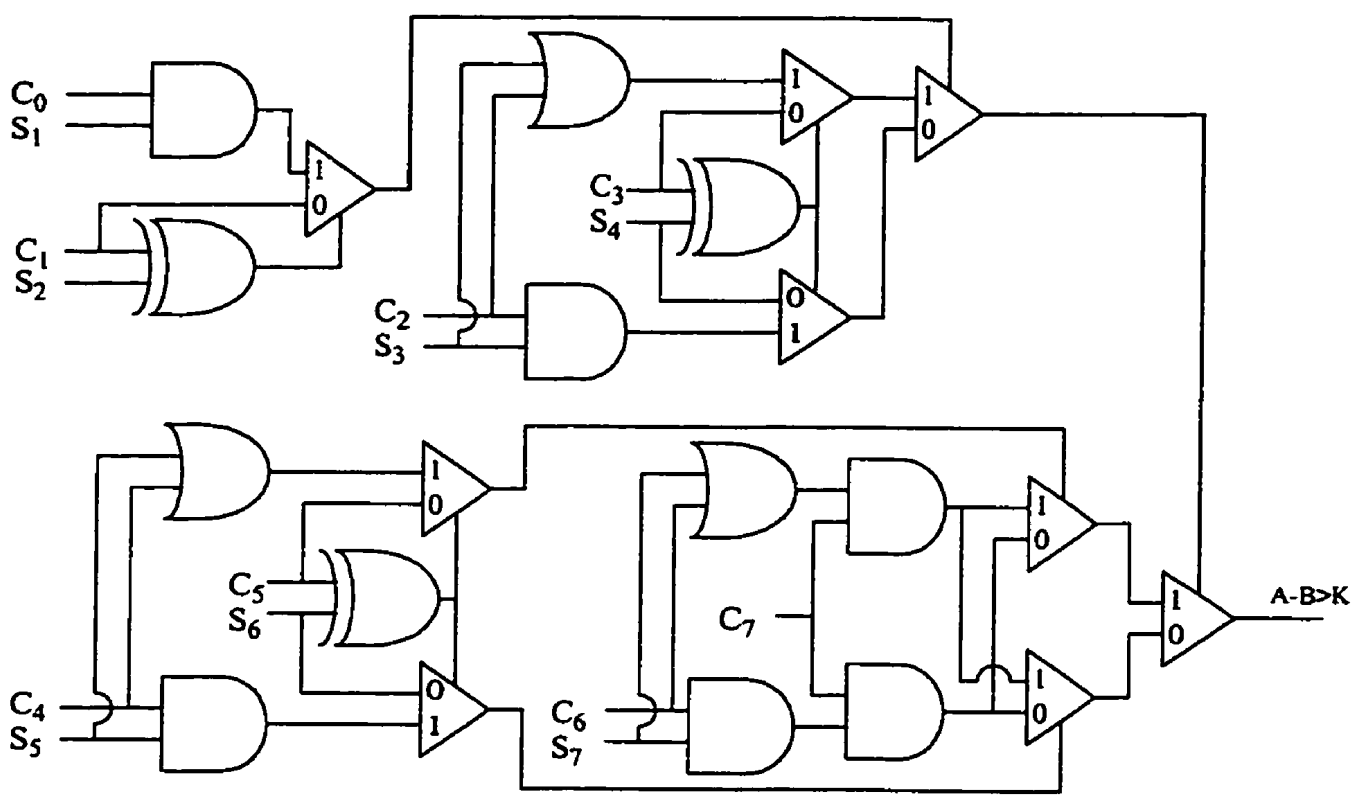

FIGURE 5.6. Carry Chain Logic for Evaluating A-B>K Condition

The signal which indicates a useful shift of the $A$ or $B$ mantissa is $S A$ or $S B$ respectively. These are generated as shown in equation (5.3) and equation (5.4), where $Z$ represents the condition that either $A$ or $B$ (or both) is zero.

$$
\begin{aligned}
& S B=(A>B) \cdot \bar{Z} \cdot(A-B \leq K) \\
& S A=(B>A) \cdot \bar{Z} \cdot(B-A \leq K)
\end{aligned}
$$

$S A$ and $S B$ will not be asserted when one or both exponents are zero, the difference $|A-B|$ is beyond the shift range or the exponents are equal. If either $S A$ or $S B$ is true (they are mutually exclusive), then the shift fabric is required to shift the $A$ or $B$ mantissa respectively. 
With the generation of the control signals to determine which, if any, of the input mantissas is required to be shifted and by how much, the presentation of the signals to the switch fabric must still be considered. To prevent unnecessary switching, the mantissa value and control signals must not be presented to the switch fabric prior to the determination of a useful shift condition. The signals for a useful shift, namely $S A$ and $S B$, may be used to control the input latches of the shift fabric. In order to prevent the latching of unnecessary values, the $S A$ and $S B$ signals can gate a delayed version of the system clock. The clock must be delayed sufficiently so that the $S A$ and $S B$ signals are valid for the current input data before the rising edge of the delayed clock as shown in Figure 5.7. If either

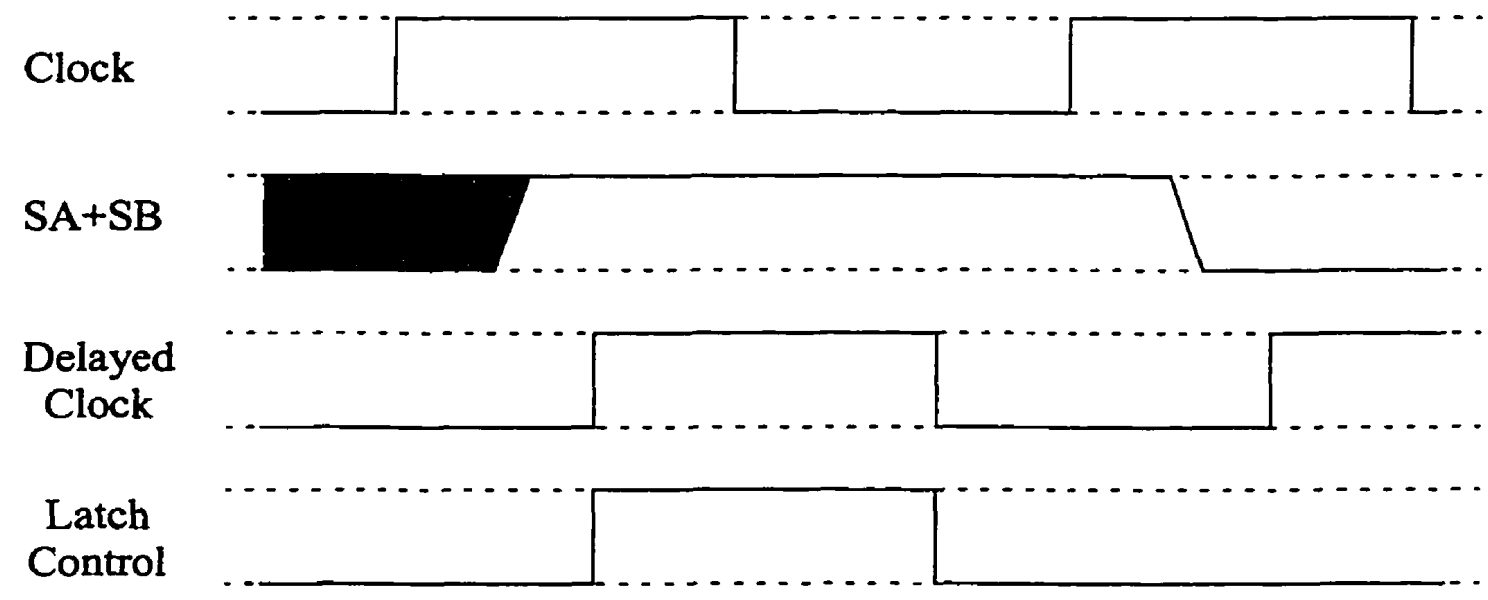

\section{FIGURE 5.7. Switch Fabric Latch Control}

$S A$ or $S B$ is true, then the mantissa for shifting and shift value are latched at the inputs to the switch fabric as shown for the first clock pulse in the Figure. If both are false then the rising edge of the clock will be suppressed and the input latches to the switch fabric will retain their past values as shown in the second clock pulse. This eliminates the unnecessary switching activity for cases where the switch fabric is not required. 
The outputs of the alignment sub-block are controlled by the same signals that determine the shift conditions. The working exponent for the block floating point pipeline is the largest of the two input exponents. For each output mantissa the proper selection is determined from the $S A, \mathrm{SB}, A>B, A-B>K$ and $B-A>K$ control signals. The possible output for each of the mantissas is the input mantissa unchanged, a shifted version of the input mantissa or a forced zero value.

\subsubsection{Alignment Sub-Block Version II}

Version II of the Alignment sub-block of the preprocessor, shown in Figure 5.8, performs the mantissa alignment of the $x$ and $y$ input values and the selection of the working exponent without using the activity scaling used in Version I. This version targets implementations with smaller mantissa and exponent widths which are statistically less

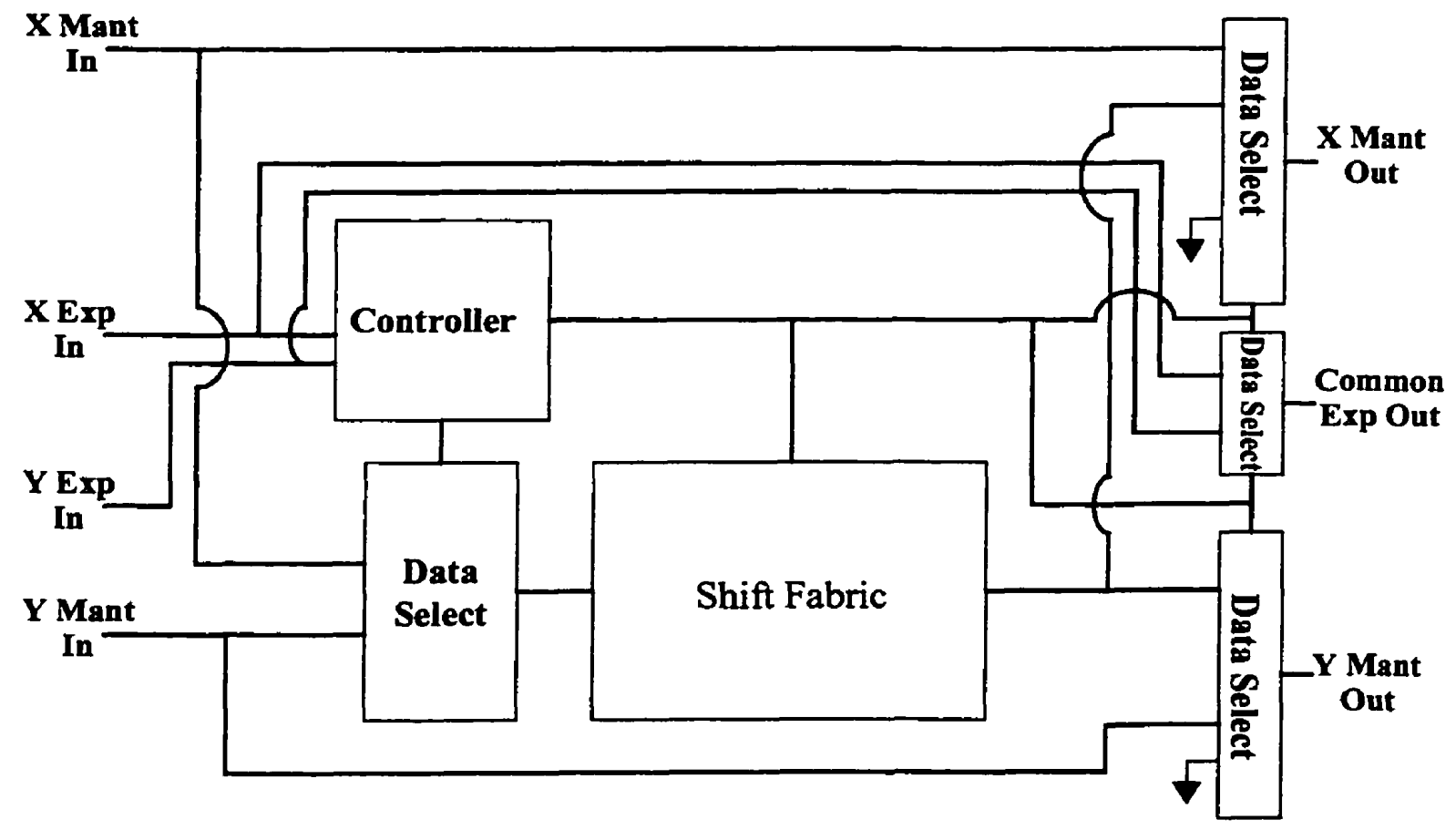

FIGURE 5.8. Preprocessor Alignment Block - Version II 
likely to have an exponent difference of more than the mantissa width. These conditions do not benefit from the bypass of the shift fabric as the area / power cost of the extra control logic and the registers at the inputs of the shift fabric may well exceed the savings achieved. This version may also be desired by designers who know the distribution and characteristics of their input signals and the probability of the shift fabric bypass condition.

In this version of the Alignment sub-block, the required control signals are reduced to the determination of $A>B,|A-B|, A=0, B=0$ and whether the exponent difference is beyond the shift range (BSR). The condition $A>B$ is determined using the carry chain of a 1 's complement adder in the same way as in Version I. The generation of the $|A-B|$ sum requires the determination of all bits since, unlike Version $\mathrm{I}$, there is no $A-B>K$ condition to indicate when the sum is not required. There is no upper limit on the sum and

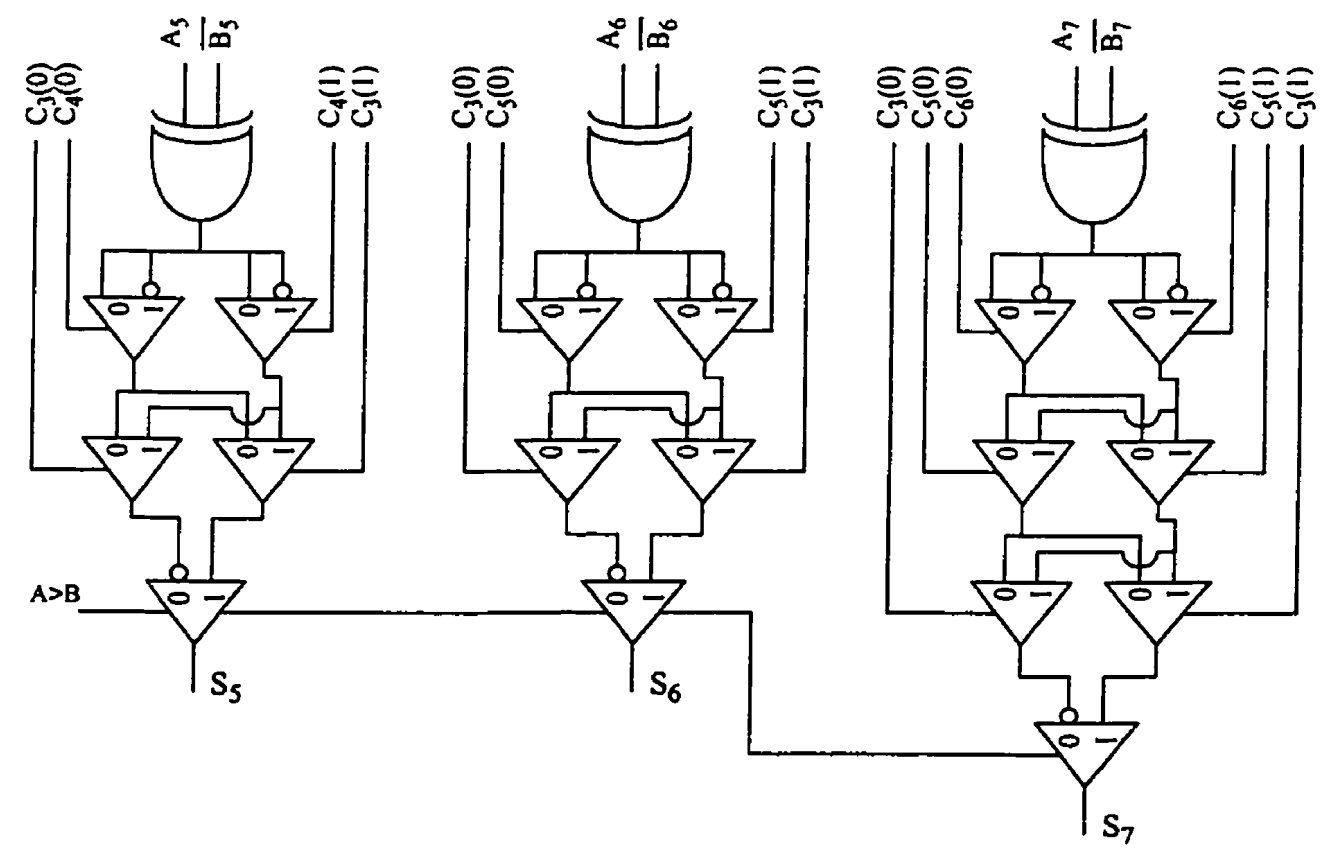

FIGURE 5.9. Generation of S5, S6 and S7 of $|A-B|$ Computation 
so all bits are required to determine the value $|A-B|$ and the BSR signal. The remaining bits are relatively easy to produce as shown in Figure 5.9. The BSR condition can then be determined by performing a comparison of the sum bits with the constant value of the largest useful shift, which is still the mantissa width -1 .

The selection of input data for the shift fabric is controlled by the $A>B$ signal. If the condition is true then the $A$ mantissa is the input, otherwise the $B$ mantissa is the input. Due to the lack of activity scaling, the inputs to the shift fabric are updated to new values for every set of input data, regardless of whether the output of the shift fabric is needed. This can lead to significant power consumption due to the delay imbalance between the data paths for the mantissas and the control signals. The mantissa to be shifted will be presented before the shift amount is available and so it will initially be shifted by the previous value before being shifted by the proper value.

The outputs of the alignment sub-block are controlled by the $A>B$ and BSR control signals. The working exponent for the block floating point pipeline is the largest exponent of the two operands $A$ and $B$. For each of the output mantissas, the proper selection is determined from the $A>B$ and BSR conditions. If BSR is false, along with a false $A>B$ condition, then the proper $A$ mantissa is the output of the switch fabric. If $A>B$ is true then the $A$ output mantissa is identical to the input mantissa. If $A=0$, or if $A>B$ condition is false and BSR is true, then this indicates that the output mantissa should be forced to all zeros. 


\subsubsection{Preproccessor Rotation Sub-Block}

The Rotation sub-block of the preprocessor is the same regardless of which version of the Alignment sub-block is used. As stated earlier, the function of the Rotation subblock is to perform the initial rotation of $\pi / 2$ radians to expand the range of convergence to $\pm \pi$ as discussed in Chapter 4. The architecture of the sub-block is shown in Figure 5.10, however, in some implementations the $z$ data path is not required.

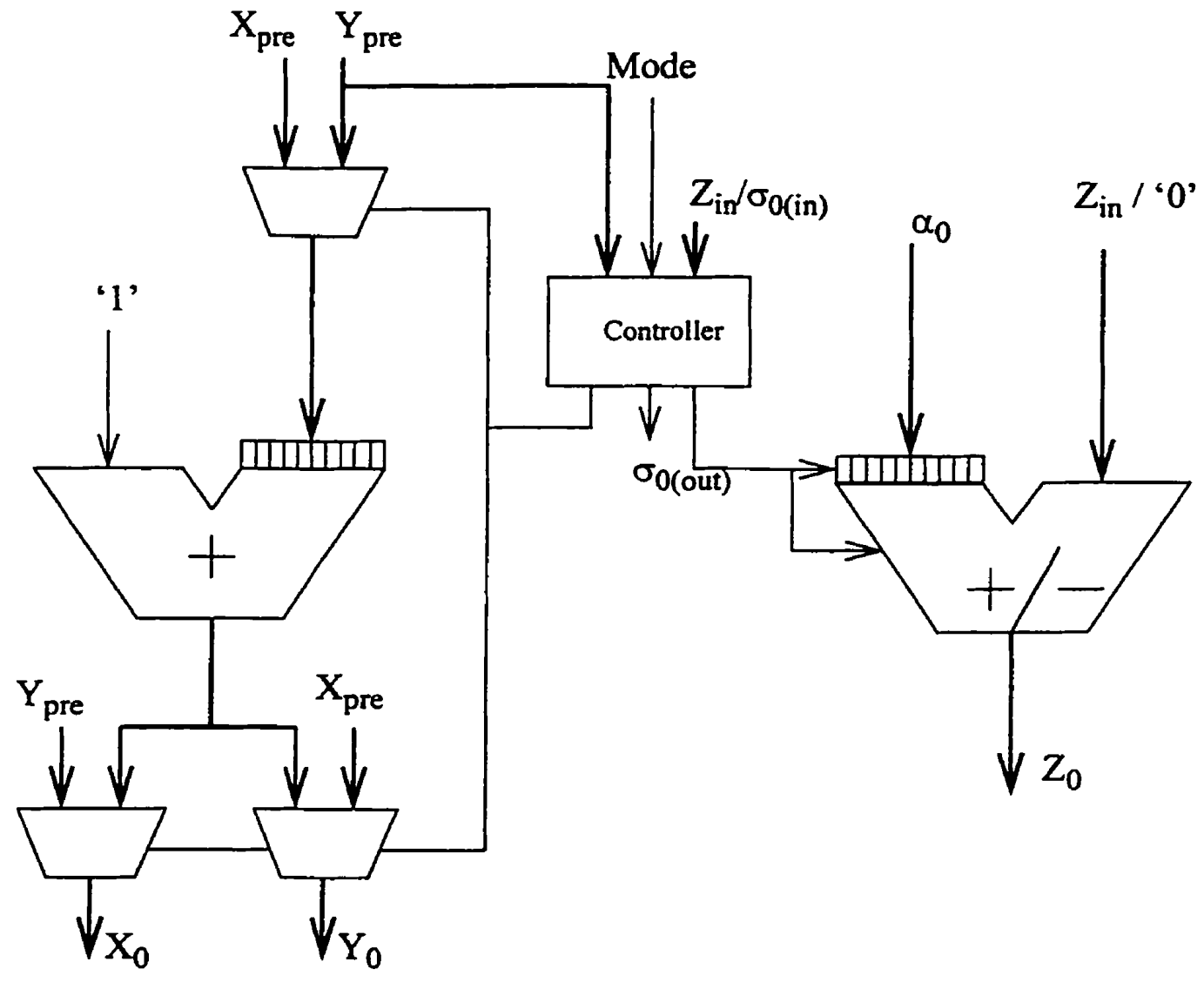

FIGURE 5.10. Preprocessor Rotation Sub-Block 
The inputs are the pre-aligned $x$ and $y$ mantissas and the angle input $z$ if present. The constant $\alpha_{0}$ is the arctan of $\pi$ radians and is used to update the angle value. The controller uses as inputs the aligned value of $y, z_{\text {in }}$ or $\sigma_{0(\text { in) }}$ depending on the mode of operation and implementation selection, to determine the proper direction of rotation. Once determined, a control signal is passed to the input multiplexers of the complementer and the output selection multiplexers for $x$ and $y$. If a rotation of $+\pi$ or $-\pi$ is required, the $x$ and $y$ values are interchanged with negation as expressed below.

$$
\begin{aligned}
\text { For }+\pi \text { rotation: } x & =-y \\
y & =x \\
\text { For }-\pi \text { rotation: } x & =y \\
y & =-x
\end{aligned}
$$

It is clear that either the $x$ or the $y$ value will need to be complemented but never both. The control signal determines which input value is complemented using an input multiplexer and assigns the proper values to the outputs using two additional multiplexers, one for each of the $x$ and $y$ operands. For the $z$ datapath, the control signal determines whether the value $\alpha_{0}$ needs to be added or subtracted from the initial $z$ value.

\subsection{Pipeline Stages}

The CORDIC algorithm computations are distributed among several pipeline stages, which can take different forms for computational efficiency. The determination of how many iterations are performed in a single stage was made based on the fact that smaller stages are preferred for generality and expandability and that the double shift iter- 
ations take approximately twice the computational time of single shift iterations. In each stage, either one double shift iteration or two single shift iterations are performed. The architectures for both the double shift and single shift stages have been developed to create a full parallel style pipeline, where each stage performs one iteration (two in the case of the single shift block) before passing the result to the next pipeline stage. Architectures have also been developed to operate in a serial/parallel style pipeline, where each pipeline stage performs a series of iterations before passing the result to the next pipeline stage. The two versions of each type of pipeline stage allow for flexible implementations based on the designer's goals with respect to timing, area and power consumption. The architecture of each of these pipeline stages will be discussed in detail in the following sections.

\subsubsection{Double Shift - Full Parallel Block}

The Double Shift - Full Parallel block performs computations in the first set of iterations for fully pipelined CORDIC processors It consists of a single block which is capable of performing the computation for one set of the parameters $S(i), S^{\prime}(i)$ and $\eta(i)$. The generic architecture of the $x$ and $y$ data path of the block is shown in Figure 5.11. This architecture is generic in the sense that all possible components of the architecture are shown although they might not be required in the final implementation. This is because the input parameters are fixed for a particular iteration of the CORDIC process, hence many portions of the architecture can be eliminated through logic optimization. Portions that may be eliminated in this manner will be indicated as they are discussed.

Inputs to the block are the parameter set, the mode of operation, either rotation or vectoring, the previous stage's output angle or the angle set value depending on the desired 
angle representation, and the previous stage's $x$ and $y$ values. The $x$ and $y$ values are shifted twice according to the parameters $S(i)$ and $S^{\prime}(i)$ with hardwired connections as the parameters are known apoiri. Recall that two extra bits are retained as part of the shift, the round and sticky bits. The two shifted values for $x$ and $y$ are respectively added or subtracted

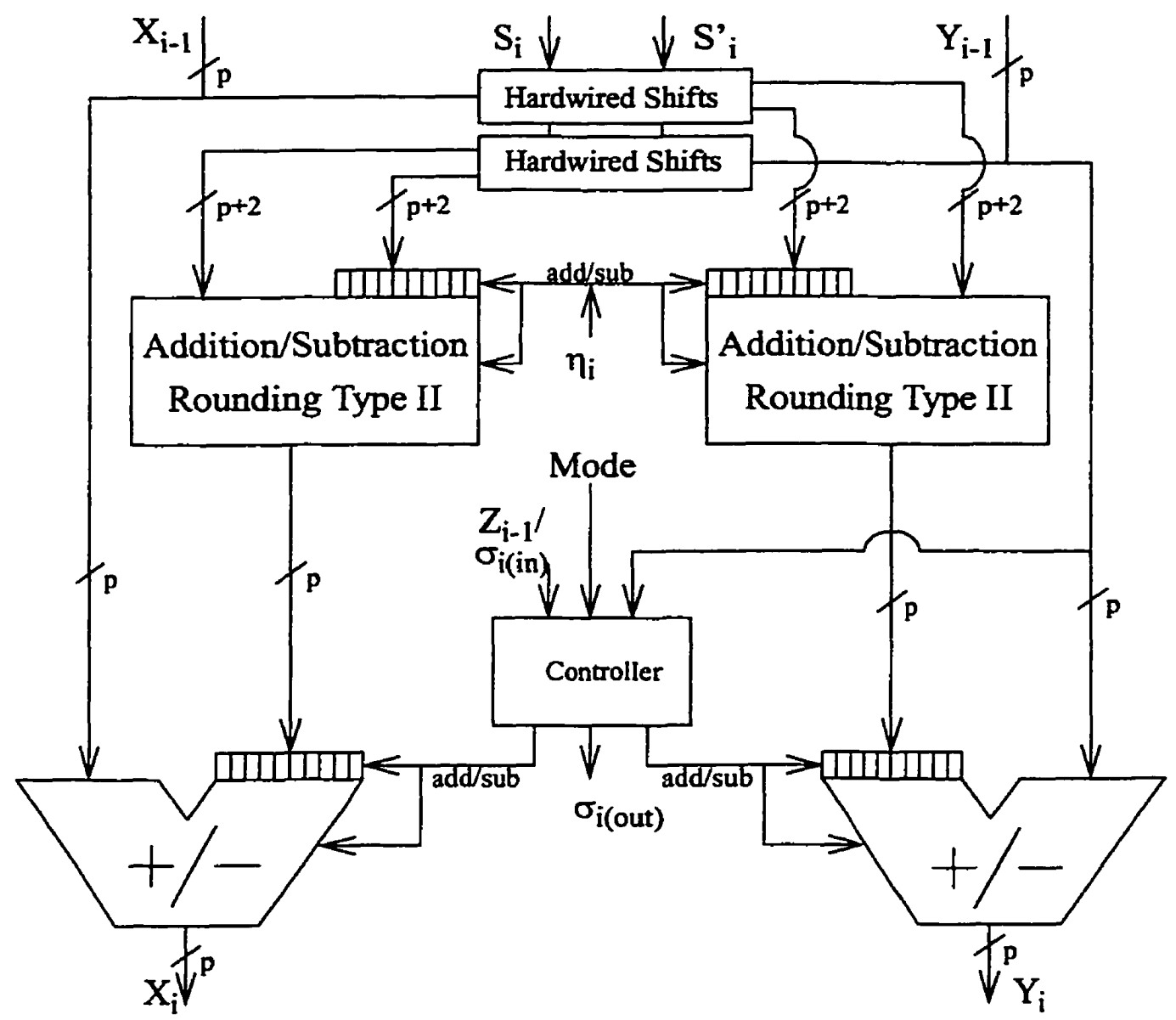

FIGURE 5.11. Double Shift Pipeline Stage - Fully Parallel Implementation

with the type of operation determined by $\eta(i)$. In the case where a subtraction is required the shifted number resulting from the shift of $S^{\prime}(i)$ is bitwise inverted and a carry in of one is provided to the adder. This effectively results in a two's complement operation. The addition/subtraction is performed according to Rounding Method Two, as outlined in section 4.5.2 for two shifted operands, and as described architecturally in section 5.2.6. The 
result of this operation is then summed with the previous value of $x$ and $y$ in the crossaddition manner required by the CORDIC equations. If the operation is a subtraction, as determined by the mode of operation and input values, then the shifted value sum is bitwise inverted and a carry in of one is added. The inverters/multiplexers for the second stage adders can not be eliminated through optimization as the operation required is data dependent and not known in advance. This second adder stage does not involve a rounding strategy as neither of the operands is the result of a shift operation.

The controller determines whether the cross-addition operation is an addition or a subtraction in order to achieve the required rotation direction. This decision is based on various inputs depending on the desired implementation. If the CORDIC PE has $\sigma_{i(\text { in })}$ as an input, then the rotation direction is available and need not be determined. Otherwise the sign of $y_{i-1}$ or $z_{i-1}$, depending on the mode of operation, determines the direction of rotation. If the PE is required to produce the output signals $\sigma_{i \text { (out })}$ then these are available from the controller and are simply the direction of the current rotation.

The operation of the $z$ data path is shown in Figure 5.12. The value of $\alpha_{i}$ is determined by the input parameters $S(i), S^{\prime}(i)$ and $\eta(i)$ and is, therefore, known and constant for each pipeline stage. The operation to be performed, addition or subtraction, is determined by the same controller, as appears in Figure 5.11, for the $x$ and $y$ data path. As in the second stage adders of the $x$ and $y$ data path, the operation is data dependent and, therefore, the inverters/multiplexers can not be removed through optimization. Again, to achieve a 2 's complement operation a carry in of one is provided in the case of a subtraction. The 
existence of the $z$ data path is dependent on the desired implementation and is not required when operating in the rotation/angle set mode. In this case the $z$ data path portion of this pipeline stage, as with the others, is eliminated thus saving considerable area and power.

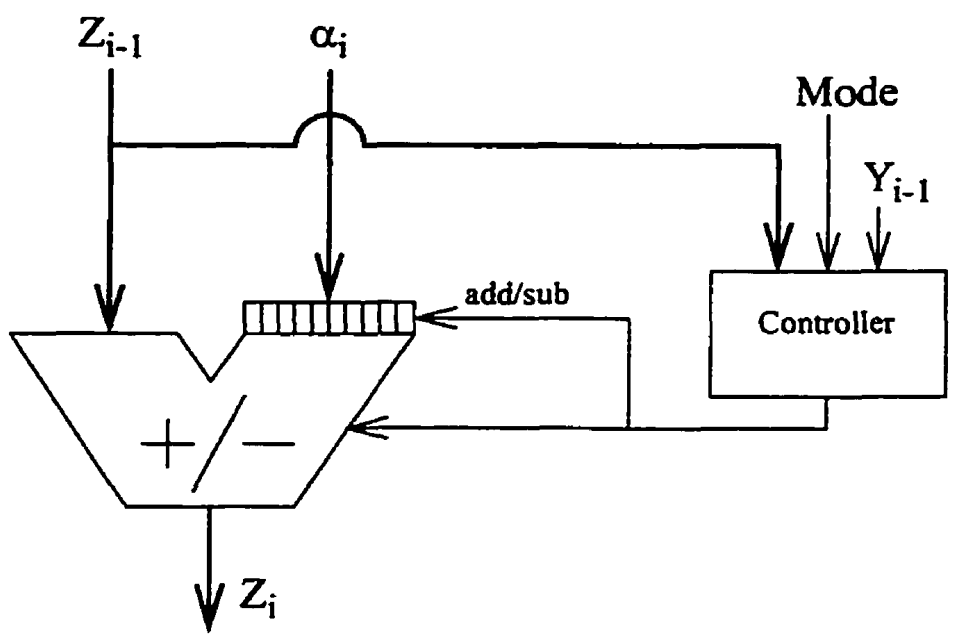

FIGURE 5.12. Z Data Path - Double Shift Pipeline Stage

\subsubsection{Single Shift - Full Parallel Block}

The Single Shift - Full Parallel block performs computations in the final set of iterations in the fully pipelined implementations of the CORDIC processor. A single block is capable of performing the computation for two successive iterations using two sets of parameters. The computation time to process these iterations matches, as closely as possible, that of the double shift block. The generic architecture of the $x$ and $y$ data path of the block is shown in Figure 5.13. Again, this architecture is generic in the sense that all possible components of the architecture are shown although they might not be required in the final implementation. In the case of the single shift block, however, the possibility of opti- 
mization is limited due to the data dependency of the operations and is in fact restricted to the controllers.

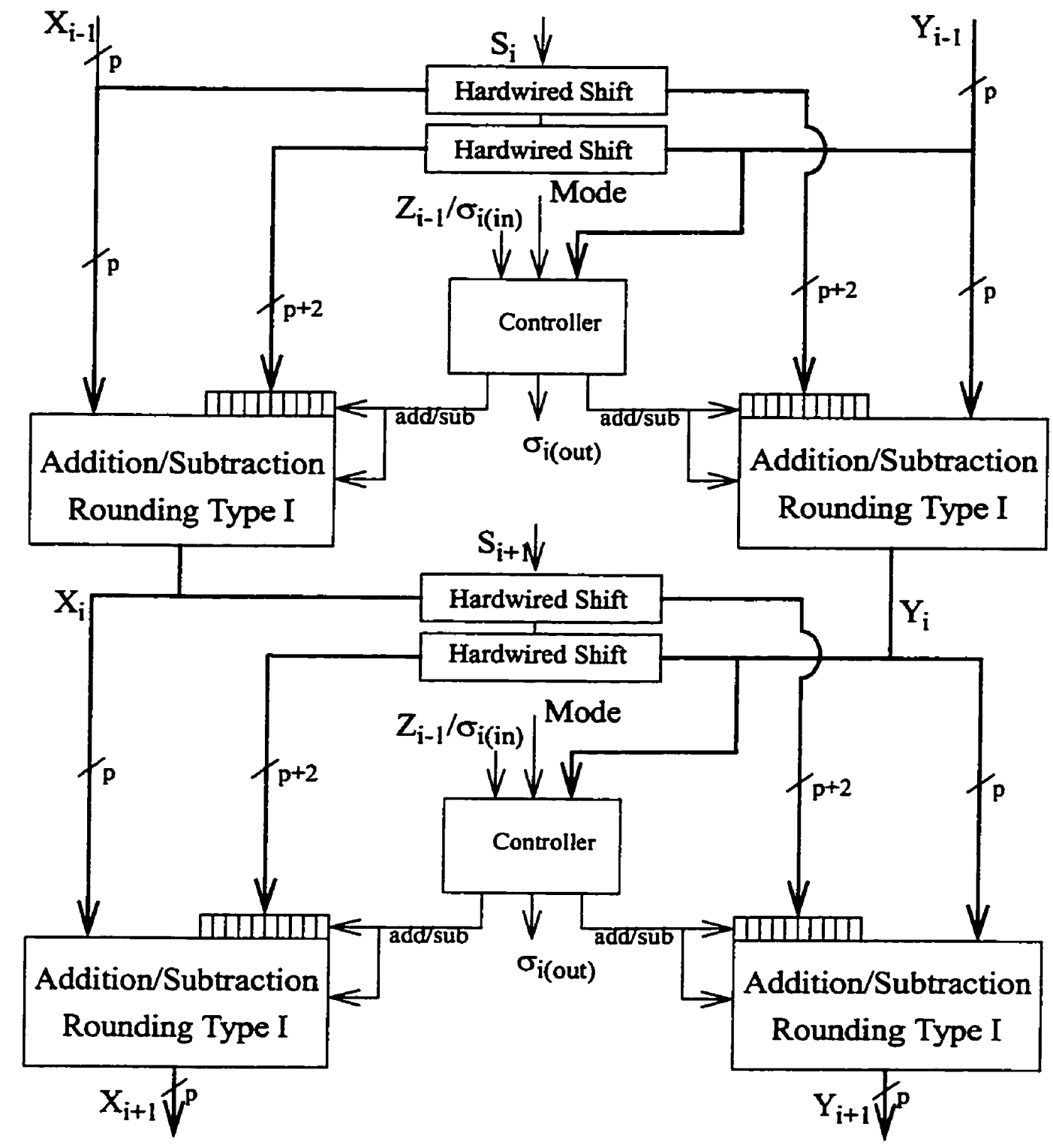

FIGURE 5.13. Single Shift Pipeline Stage - Fully Parallel Implementation

Inputs to the block are the parameter set, the mode of operation, either rotation or vectoring, the previous stage's output angle or $\sigma_{i(i n)}$ and the previous stage's $x$ and $y$ values. The $x$ and $y$ values are shifted once according to $S(i)$ with a hardwired connection as 
the parameter is known apoiri. Again, two extra bits are retained as part of the shift, the round and sticky bits. The controller determines whether the cross-addition operation is an addition or a subtraction in the same manner presented for the double shift block. In all cases, the unnecessary inputs and outputs and the corresponding logic are removed during the optimization phase of the synthesis process. In the case where the controller deems a subtraction is required, the shifted number is bitwise inverted and a carry in of one provided to the adder for the 2's complement operation. The addition/subtraction is performed according to rounding Method One, as outlined in section 4.5.1 for a single shifted operand, and as described architecturally in section 5.2.5. The output of the first addition/ subtraction operation is the result of the first of the single shift iterations. The architecture of the second iteration of the stage is a duplication of the first with inputs from the first iteration and with the corresponding parameter.

The operation of the $z$ data path is shown in Figure 5.14. The architecture and operation are identical to the $z$ datapath of the double shift pipeline stage with the exception that it is repeated for the second iteration. The input values of $\alpha_{i}$ and $\alpha_{i+1}$ are required to update the angle. These values are pre-determined constants as are the parameters $S(i)$ and $S(i+1)$ for each pipeline stage. As with the double shift stage, the existence of the $z$ data path itself is dependent on the desired implementation and is not required when operating in the rotation/angle set mode. In this case the $z$ data path portion of this pipeline stage, as with the others, is eliminated. 


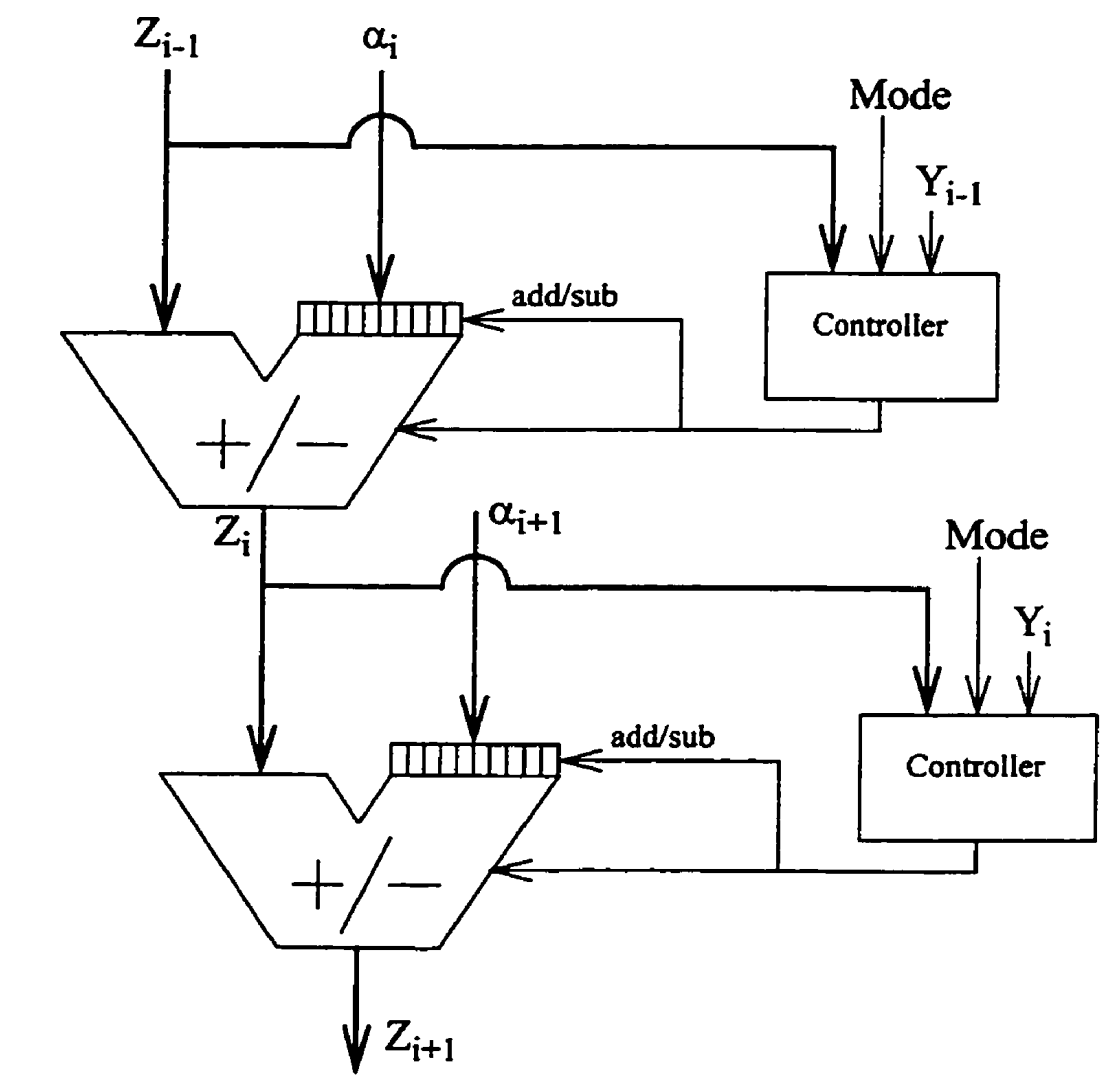

FIGURE 5.14. Z Data Path - Single Shift Pipeline Stage

\subsubsection{Double Shift FSM - Parallel/Serial Block}

The architecture of the Double Shift FSM block is shown in Figure 5.15. This pipeline stage is intended for use in parallel/serial implementations of the CORDIC PE and is capable of performing a configurable number of double shift iterations. As with the other stages, the Figure provides a generalized architecture which will be further optimized. In the case of the double shift FSM block, the optimization is based on similarities between the various parameter sets and not on one set as in the full parallel version of the block. Any possible optimizations will likely consist of small scale logic minimization, however, larger scale optimizations are possible in some cases. An example of a large 
scale optimization is the elimination or reduction of the first set of inverters/multiplexers allowed when all $\eta(i)$ parameters for the block have the same value. The computations of the double shift FSM block are the same as for the fully parallel version.

The basis behind the FSM approach is the provision of feedback within the pipeline stage to allow the results of one iteration to be used as the inputs to the next. There is obviously a hardware cost associated with the feedback path and with the FSM controller required to operate the stage properly. It is not envisioned that this version of the double shift pipeline stage will be used for performing fewer than three successive iterations as the hardware costs for the FSM block is similar to that when two full parallel versions of the stage are abutted together without any intermediate pipeline registers. A savings in area is achievable at the expense of throughput by using the serial/parallel approach to perform three or more successive iterations. The extra hardware required for this version of the double shift stage consists of a controller, three sets of multiplexers and a set of feedback registers.

The FSM controller has $s+1$, states where $s$ is the desired number of successive iterations to be performed within the stage. Initialization is performed during the first state followed by the computational iteration states. The FSM is provided with a clock whose period is $1 / s$ that of the master clock controlling the pipeline registers. This allows the data from the last iteration to be available for capture by the output pipeline registers. In each state, the FSM provides the control signals to the input multiplexers, the shift selectors and the first set of adder/subtractors. The input multiplexers select the results captured by the input pipeline registers during the first iteration and select the results captured by the feed- 


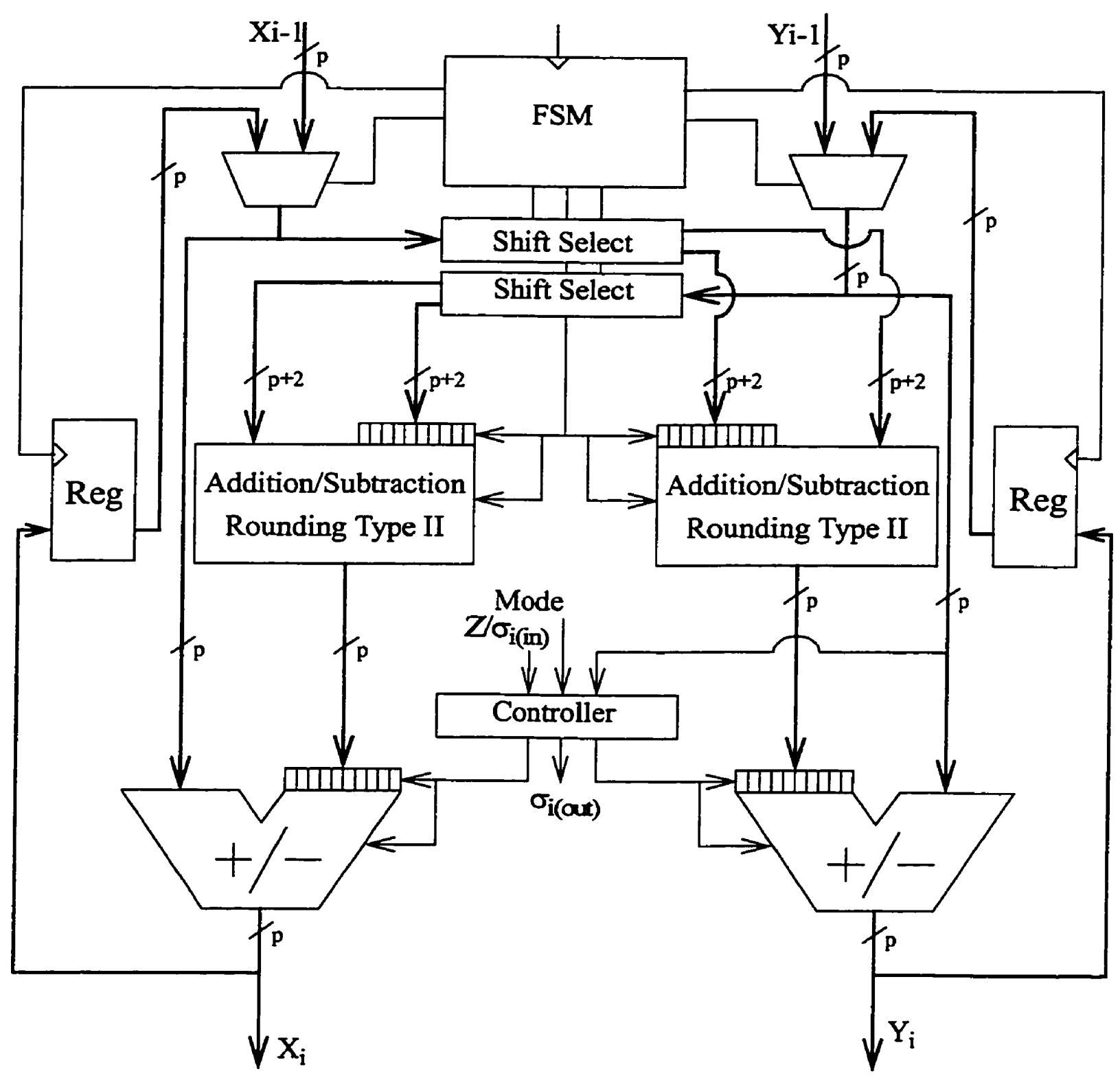

FIGURE 5.15. Double Shift FSM Pipeline Stage - Parallel/Serial Implementation

back registers in the remaining iterations. The shift selectors are multiplexers which choose between $s$ required shifts, for the $s$ iterations, each of which is hardwired. The use of hardwired shifts is advantageous as far as area and timing are concerned when compared to barrel shifters. Although this does result in additional loading on the feedback 
registers, such a load does not present a problem unless $s$ is excessively high, which would require a large number of shifts to be produced. The FSM controller also provides a gated clock to the feedback registers which results in power savings. The feedback registers need not capture the output data when leaving the reset state, and likewise at the end of the last iteration state, as the data is not required for the following iteration. Finally the FSM controller selects the proper addition/subtraction signal to the first set of adder/subtractors as required by the appropriate $\eta(i)$.

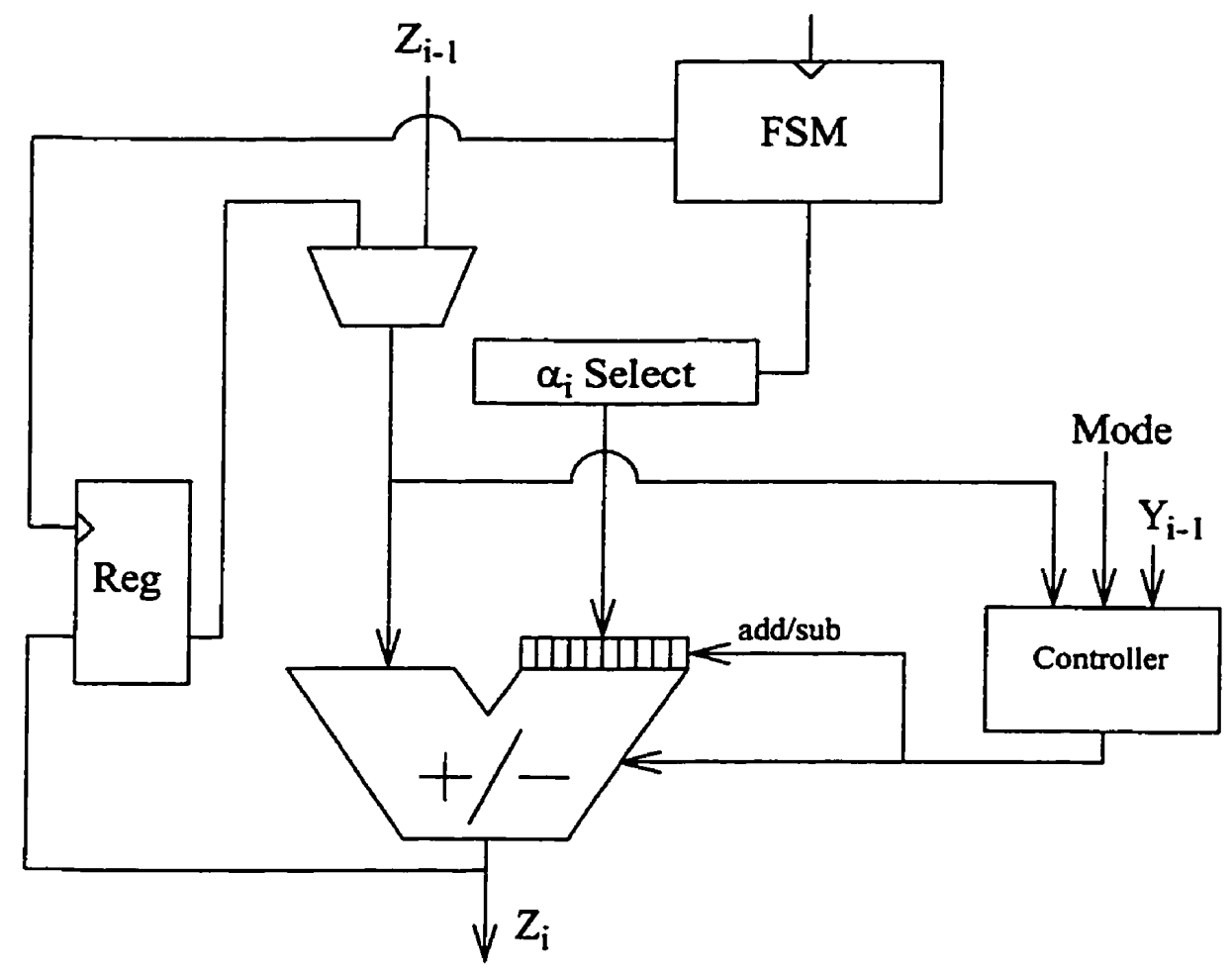

FIGURE 5.16. Z Data Path - Double Shift FSM Pipeline Stage

The $z$ datapath of the double shift FSM stage is shown in Figure 5.16. Its architecture follows the same principles as the $x$ and $y$ datapath and is based on the full parallel version of the $z$ datapath. The additional components required are two sets of multiplexers and a set of feedback registers. The detailed operation of the $z$ datapath is the same as pre- 
sented above with multiplexers to select between the pipeline or feedback register values and to present the proper $\alpha_{i}$ to the adder/subtractor. As with the other $z$ datapaths, this one may also be eliminated if it is not required for a particular implementation of the CORDIC PE.

\subsubsection{Single Shift FSM - Serial/Parallel Block}

The architecture of the Single Shift FSM block is shown in Figure 5.17. This pipeline stage is intended for use in serial/parallel implementations of the CORDIC PE and is capable of performing a configurable, but even, number of single shift iterations. As with the other stages presented, the Figure provides the general architecture. For the reasons outlined in the discussion of the full parallel single shift stage, any optimizations are restricted to the controllers as all other components are data dependent. The basic architecture of the single shift FSM block is the same as for the fully parallel version and that discussion will not be duplicated here. There are, however, a number of additional requirements for the FSM version and a discussion of these will form the remainder of this section.

The basis for the FSM approach is the same as for the double shift FSM and consists of the provision of feedback within the pipeline stage to allow the results of one pair or iterations to be used as the inputs to the next. For the same reasons as the for the double shift FSM stage, it is not envisioned that this version of the single shift pipeline stage will be used for performing fewer than three successive iteration pairs. A saving in area is achievable at the expense of throughput by using the serial/parallel approach to perform 


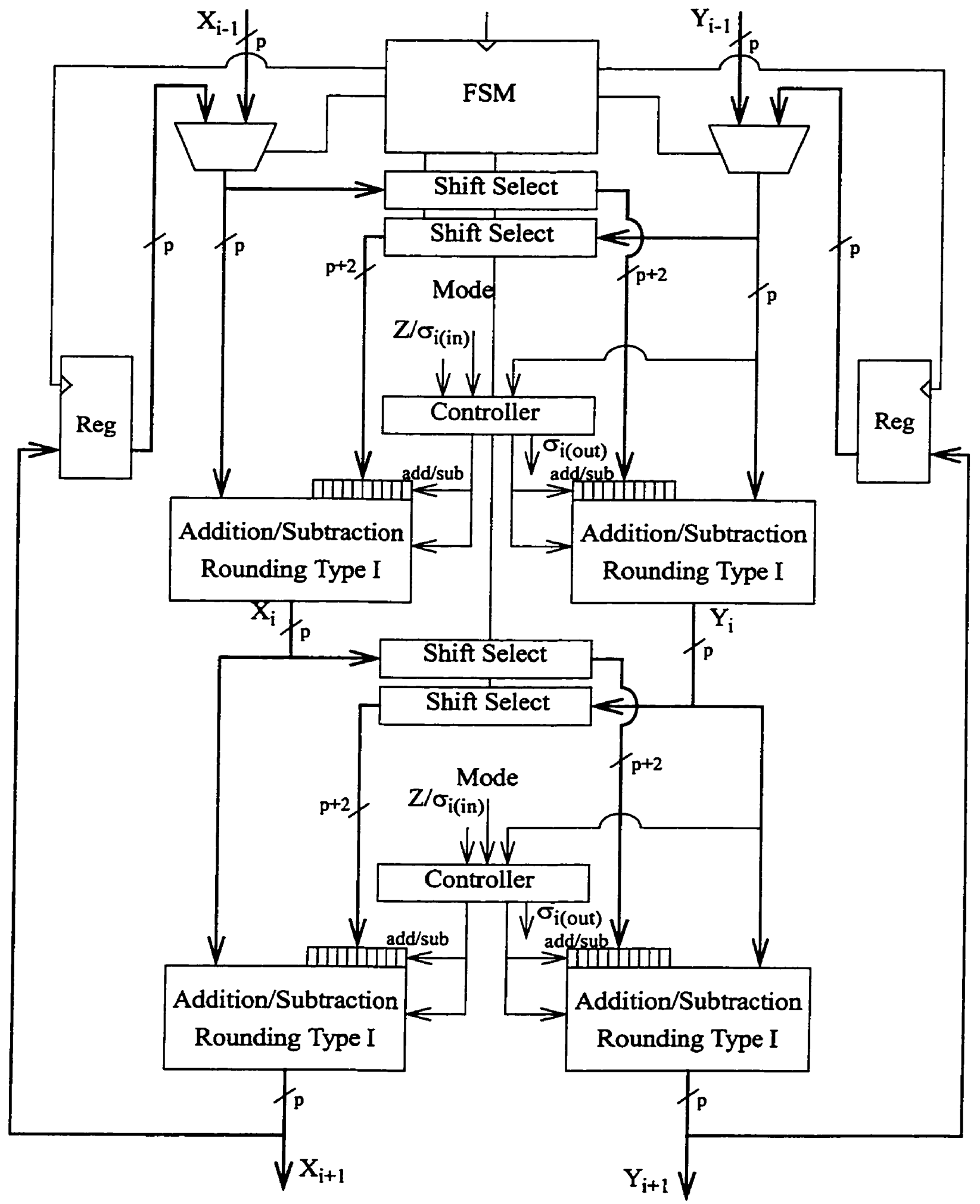

FIGURE 5.17. Single Shift FSM Pipeline Stage - Parallel/Serial Implementation 
three or more successive iteration pairs. The extra hardware required for this version of the single shift stage consists of an FSM controller, five sets of multiplexers and a set of feedback registers. The FSM operates in the same manner as the double rotation stage.

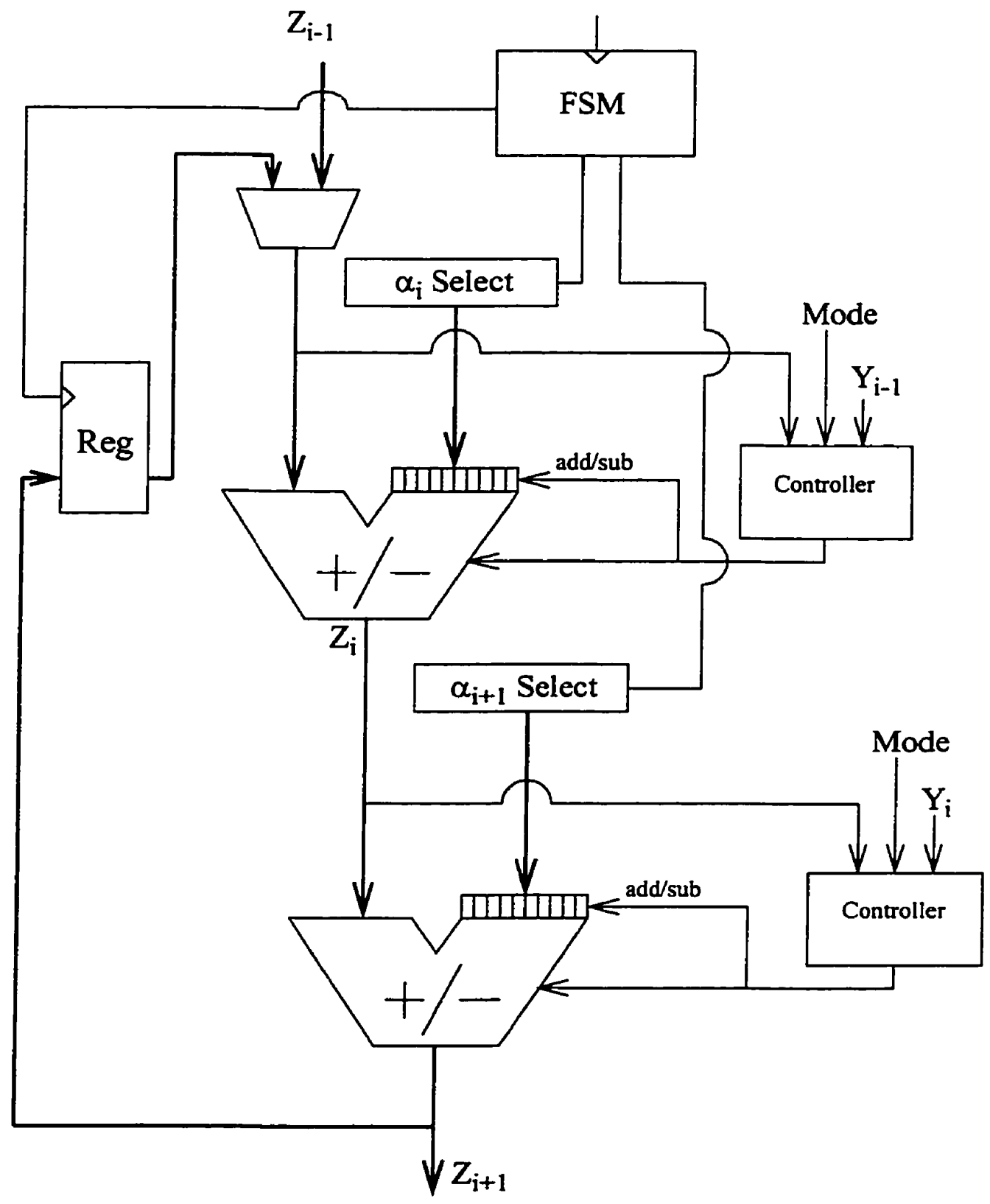

FIGURE 5.18. Z Data Path - Single Shift FSM Pipeline Stage 
The $z$ datapath of the single shift FSM stage is shown in Figure 5.18. Its architecture follows the same principles as the $x$ and $y$ datapath and is based on the full parallel version of the $z$ datapath. The additional components required are three sets of multiplexers and a set of feedback registers. The FSM controller is the same one which is used in the $x$ and $y$ datapath architecture as are the two rotation direction controllers. The detailed operation of the $z$ datapath is the same as for the double shift pipeline stage. As with the other $z$ datapaths this one may also be eliminated if it is not required for a particular implementation of the CORDIC PE.

\subsubsection{Addition/Subtraction Rounding Type I}

The architecture for the adder/subtractor which implements rounding Method One, as described in section 4.5.1, is shown in Figure 5.19. The first of the two operands is nonshifted with a width of $p$ bits. The second operand, formed from a shifting operation, has a width of $p+2$ bits and has already been inverted if a subtraction operation is desired. The carry in bit is a one for subtraction and a zero for addition. The most significant $p-1$ bits from both operands are summed in the upper block adder. The lower bits are summed separately in the lower block adder after the non-shifted operand has been appended with the appropriate bits. The result of the lower block is used by the rounding logic to determine the rounding decision which results in the proper carry in to the upper block. This decision is achieved with simple decoding logic to cover all possible cases. The impact on overall timing caused by the delay in getting the rounding decision can be minimized if a carry select or conditional sum adder type implementation is chosen for the upper block adder. This allows the selection of the proper result and does not cause a ripple like recomputa- 
tion. A carry select type adder suffers from a large increase in area and power and may not be justified in all applications. The final output consists of the appropriate result from the upper block appended with the proper LSB as determined by the rounding logic which together form a p bit number.

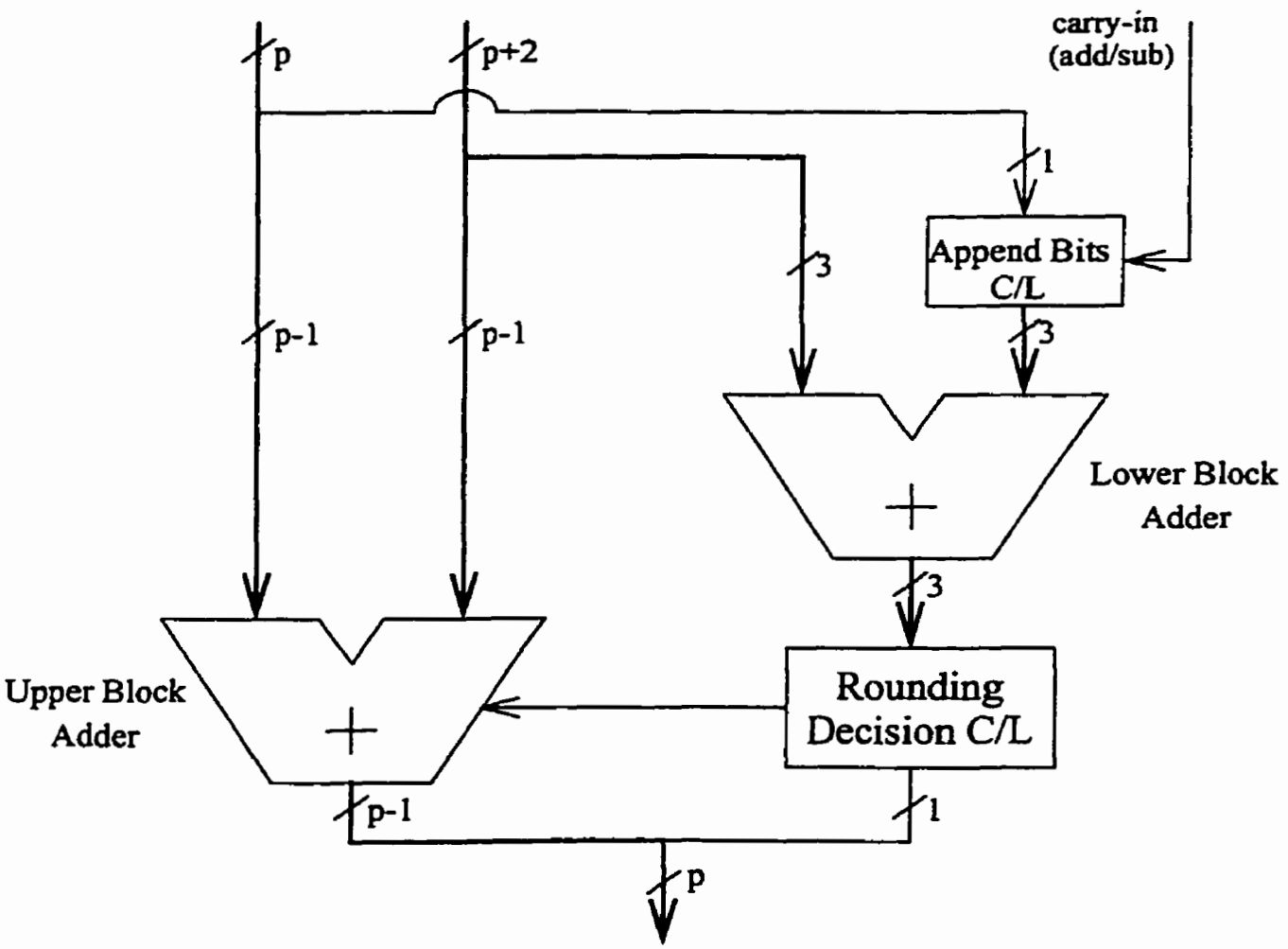

FIGURE 5.19. Addition/Subtraction Rounding Type I - One Shifted Operand

\subsubsection{Addition/Subtraction Rounding Type II}

The architecture for the adder/subtractor which implements rounding Method Two, as described in section 4.5.2, is shown in Figure 5.20. In this architecture both operands have a width of $p+2$ bits as they are both formed by shift operations. If a subtraction is required the second operand is already inverted and the carry-in signal is set to one. 


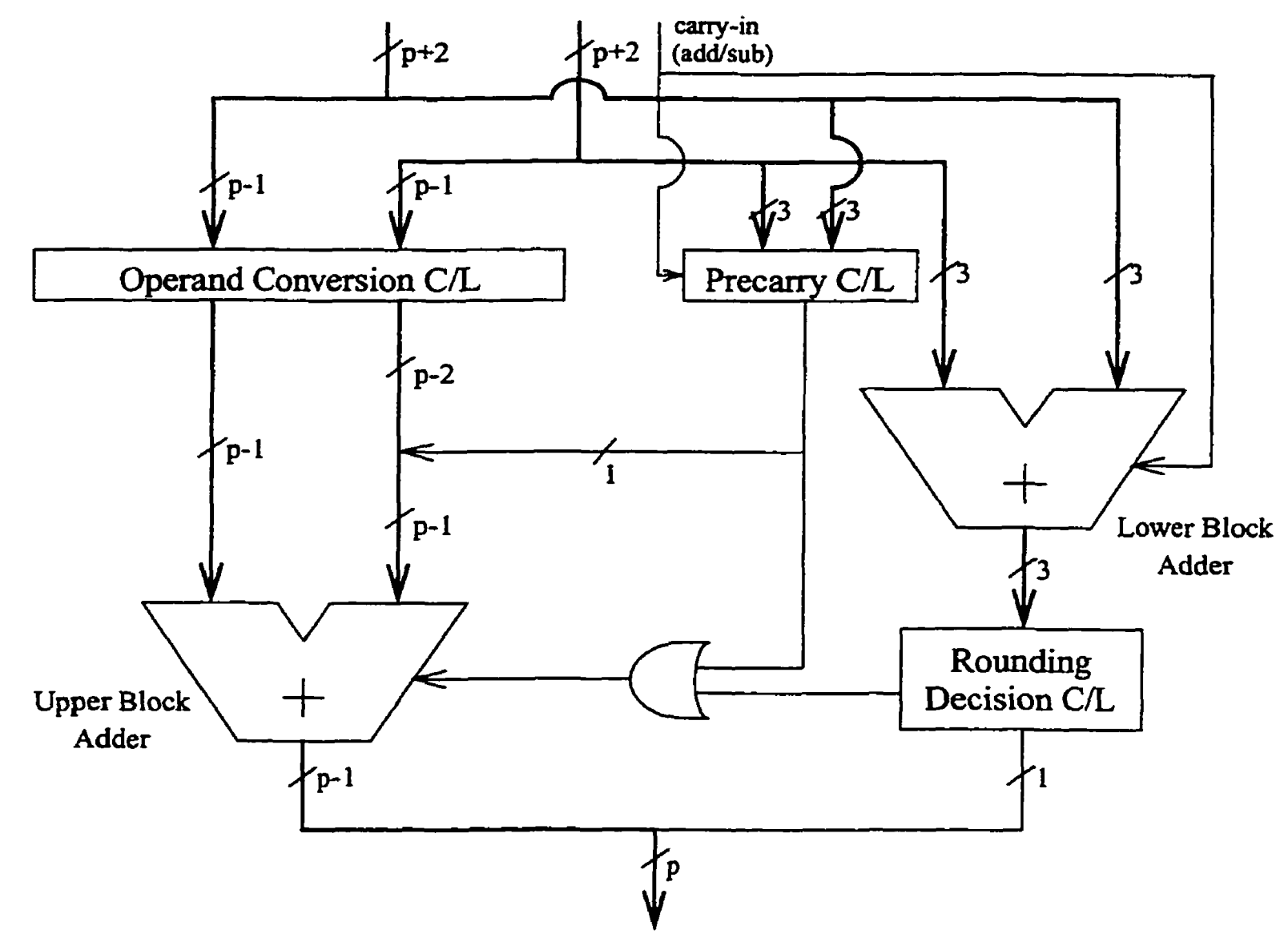

FIGURE 5.20. Addition/Subtraction Rounding Type II - Two Shifted Operands

The least significant three bits are summed in the lower block adder. Concurrently, these bits are also decoded by the precarry detection logic in order to determine if the precarry condition exists. The most significant $p-1$ bits of the operands are converted into a sum and carry vector using a single level of AND and XOR gates respectively. The carry vector is aligned with the sum vector by offsetting it by one bit to the left. The most significant bit is dropped as it is not required (recall that the data path has already been padded to handle possible overflow) and the least significant bit, which is vacant after the align- 
ment, is filled with the precarry condition. These vectors then become the inputs to the upper block adder. The rounding logic uses the results from the lower block adder to determine the rounding logic and the appropriate carry in to the upper block is provided. Again, to limit the impact on overall timing caused by the delay in the rounding decision, a carry select or conditional sum adder is preferred for the upper block summation. As with Method One, the final result consists of the output of the Upper Block adder with a bit appended from the rounding logic.

\subsection{Postprocessor}

The postprocessor performs the compensation for the scaling factor and any desired normalization. As presented in Chapter 4, the compensation for the scaling factor for the modified CORDIC equations can be performed by a simple shift. As part of the micro-rotations, the mantissa could have increased in relative magnitude by a factor of approximately 1.4 or, in other words, one bit position at the most significant end. If such a condition occurs, the postprocessor increases the block exponent by one. When the final mantissa bits have been selected the postprocessor performs the required rounding. As part of this architecture two versions of the postprocessor block have been developed. In version I there is no normalization shift. In version II there is a normalization shift to remove any sign extension bits up to the limit allowed by the exponent. This is to say that the mantissa will be normalized to the greatest extent possible while ensuring that the exponent remains valid. The architecture of the first version targets implementations where the output of one CORDIC PE is the input to another as occurs in a systolic array configuration. In these implementations the post processor normalization would be followed by a subse- 
quent preprocessor alignment thereby possibly reversing the normalization. In addition, there is an area / power cost of normalization and in some cases it may not be required by the designer. It is, of course, possible to mix the two implementations in a systolic array by having only the last stage of PEs perform normalization while the intermediate ones do not. The two versions of the postprocessor block are examined in the following sections.

\subsubsection{Postprocessor Version I}

Postprocessor version I does not perform the optional normalization of the $x$ and $y$ results. It takes as inputs the $x$ and $y$ mantissas from the last pipeline stage and the working exponent, as determined by the preprocessor and transferred through the pipeline in parallel with the corresponding input data. As stated, the postprocessor will compensate for the scaling factor as well as any bitwise magnitude growth that occurred. The architecture for the $x$ data path is shown in Figure 5.21, while that of the $y$ data path is completely analogous.

The input mantissas are stripped of the error accumulation bits from the least significant part before entering the postprocessor, but they still retain the two overflow bits. Recall that one of the overflow bits was required due to the growth in magnitude by two caused by the scaling factor. The second overflow bit was to account for a magnitude growth with an upper bound of two which could result from the normal rotation operations. The compensation of the scaling factor can be accomplished by a shift of the mantissa by one bit position to the right. Instead of actually performing a physical shift, it is permissible to simply select the mantissa starting one bit position to the left. For this implementation, it means choosing the proper number of bits with the MSB being the least 


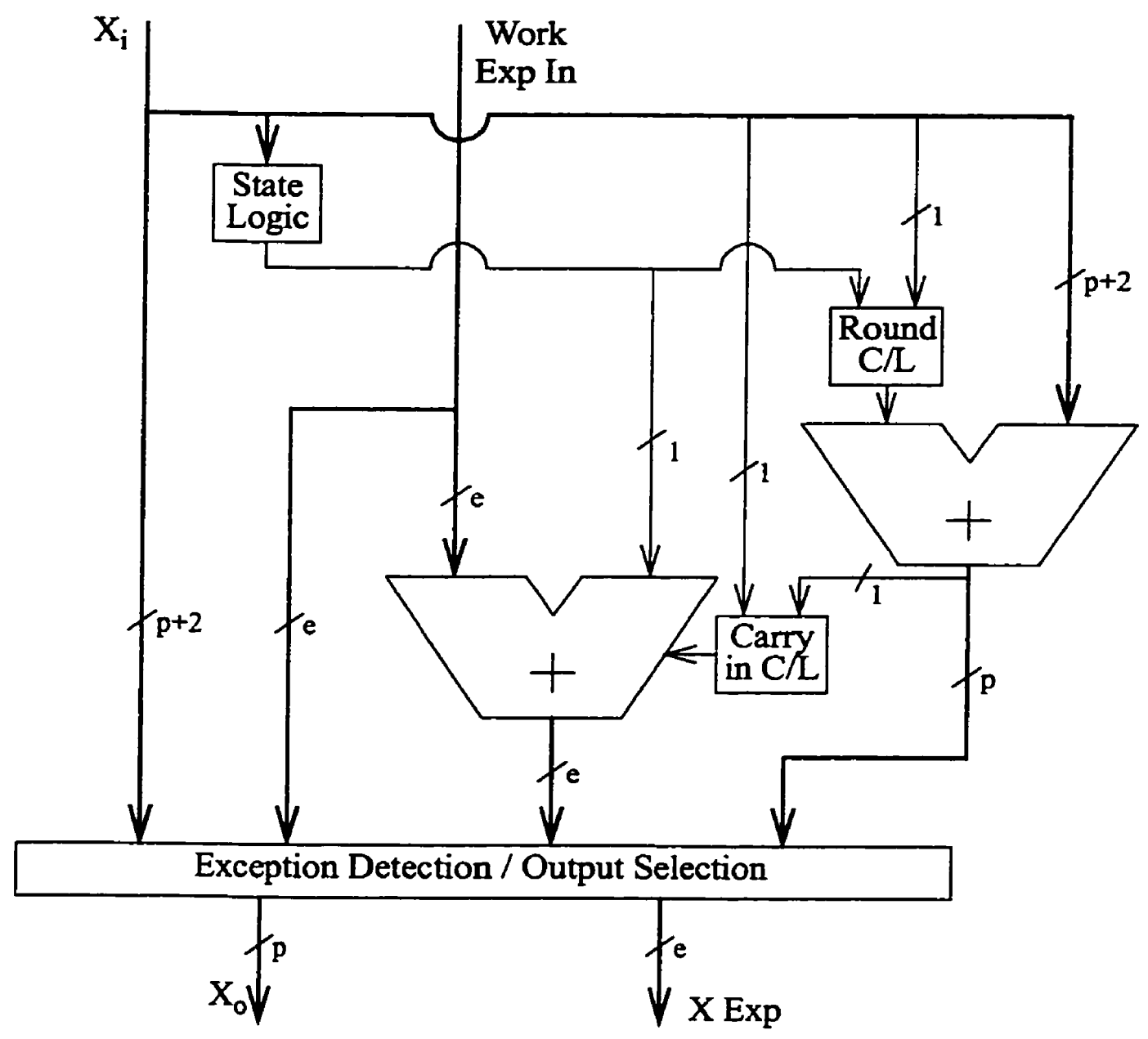

FIGURE 5.21. Post Processor without Full Normalization (X Data Path)

significant of the overflow bits. This situation is shown graphically in Figure 5.22, where the mantissa $M$ has $n$ bits, $S$ is the sign bit and $O_{G}$ and $O_{S}$ are the overflow bits for growth and scaling respectively. The exponent is unaffected by this pseudo shift as the scaling effect occurred in the mantissa computation iterations while the exponent was unchanged. If the $O_{G}$ bit is significant and is, therefore, required in the final result, then this will require an increase in the exponent. 


\section{Original Mantissa Bit Positions}

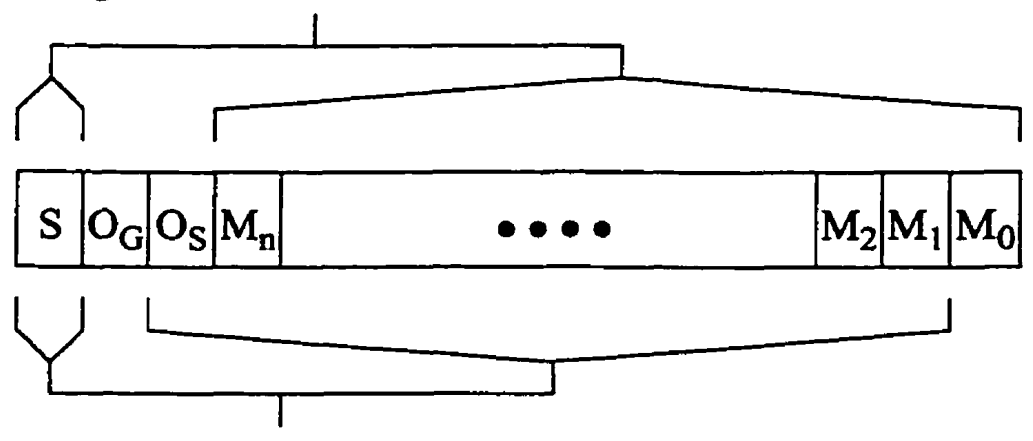

\section{Resultant Mantissa Bit Positions}

\section{FIGURE 5.22. Pseudo Shift Selection of Mantissa Result Bits}

The execution of the Post-Processor depends on the mantissa value. Evaluation of the mantissa is performed in the State Logic to determine if the mantissa has undergone an order of magnitude growth during the rotation. A magnitude growth is indicated by the $O_{G}$ bit being different from sign bit $S$. If the two bits are the same, it indicates that the $O_{G}$ bit is simply a sign extension bit and is, therefore, not used. In the case where no growth has occurred, other than that to be compensated for by the scaling factor, then the selection of the proper mantissa bits is all bits except the $S$ and $M_{0}$ bits as shown in Figure $5.23 \mathrm{a}$. The $S$ bit may be discarded as a sign extension bit and the $O_{G}$ bit, which is the same as the $S$ bit, becomes the new sign bit. The $M_{0}$ bit becomes a rounding bit, $\mathrm{R}$. In this case there is only one bit for rounding so the second rounding bit, the guard bit, is set to 0 .

If an order of magnitude growth has occurred then the proper selection of mantissa bits is all bits except the two LSBs, which become the $R$ and $G$ bits for rounding, as shown 
in Figure 5.23 b. According to the rounding procedure previously outlined, rounding is performed if the new LSB is a 1 and either the $R$ or $G$ bits is a 1 , or if both the $R$ and $G$ bits are 1 . Rounding is not performed under other conditions.

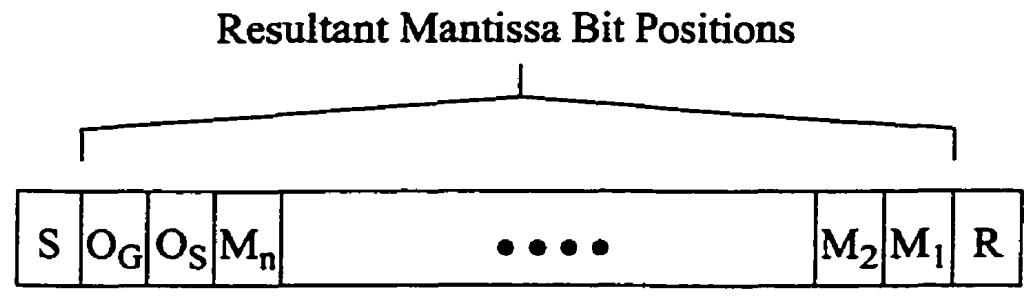

a) No order of magnitude growth

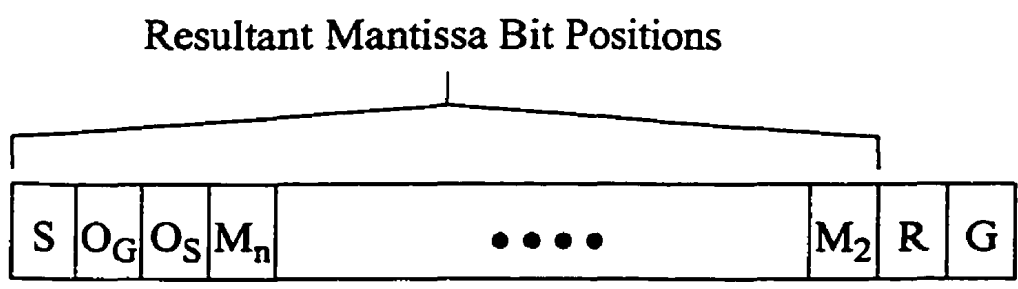

b) Order of magnitude growth

\section{FIGURE 5.23. Selection of Mantissa Result Bits - Version I}

After rounding is performed, the mantissa is checked to determine if the action of rounding caused an overflow. An overflow caused by the addition of 1 ulp is indicated by a change in the sign bit (the MSB) from a 0 to 1 . If this occurs then a new sign bit of 0 is inserted at the MSB position while the LSB is truncated to maintain the correct number of bits. The truncation of the LSB is not important since the remainder of the result must be all 0s. It is impossible for overflow to occur through the addition of 1 ulp and to have anything other than trailing zeros. 
If the mantissa result did not undergo an order of magnitude growth, and there was no mantissa overflow due to rounding, then the final exponent is equal to the working exponent. If, however, either or both of these conditions occur then the exponent must be increased by one or two depending on whether one or both of the conditions occurred. The exponent is then tested to see if after any increase it is still within the valid range of exponents. If the exponent has suffered overflow then the output exponent is forced to all is and the output mantissa to all 0 s to indicate a $+\infty$ result. Underflow can not occur as the output exponent would never be less than the working exponent, which is itself a valid exponent.

\subsubsection{Postprocessor Version II}

Postprocessor version II performs the optional normalization of the $x$ and $y$ results. It takes as inputs the $x$ and $y$ mantissas from the final pipeline stage and the working exponent, as determined by the preprocessor. The postprocessor compensates for the scaling factor as well as any bitwise rotational growth in addition to removing as many of the sign extended bits as allowed. The architecture for the $x$ data path is shown in Figure 5.26 while that of the $y$ data path is completely analogous.

\section{Normalization Cases}

With the introduction of normalization, the number of possible variations for dealing with the exponent and the mantissa increases to four principle cases. These cases, as shown in Table 5.2, are based on the number of sign extended bits in the mantissa and the value of the exponent. Case 1 occurs if there is only one sign extension bit. This indicates 
that a rotation growth by an order of magnitude did not occur and that the mantissa grew under the effects of scaling alone. Similar to one of the cases in version $I$, the selection of final resultant bits starts with the $O_{G}$ bit as it is identical to the sign bit. The LSB becomes the $\mathrm{R}$ bit used in the mantissa rounding decision. The exponent does not need to be increased or decreased in this case. This situation is shown in Figure 5.24a.

\begin{tabular}{|c|l|c|c|}
\hline Case & Sign Extension Bits & Rounding & Exponent \\
\hline 1 & One & R & No Change \\
\hline 2 & $\geq$ Two & None Reqd & - Sign Ext Bits +1 \\
\hline $2 b$ & $\geq$ Two, Work Exp $=1$ & R & No Change \\
\hline 3 & None & R, G & +1 \\
\hline
\end{tabular}

TABLE 5.2. Pre-normalization Cases

Case 2 occurs when there are two or more sign extension bits and the working exponent is greater than one. In this case, exponent allowing, the selection of the mantissa starts with the last of the sign extension bits. The LSB positions are padded with 0s, if required, in order to have the required number of bits. If the number of sign extension bits is greater than the value of the biased exponent then only possible to remove some of the sign bits. Recall that the removal of the $O_{G}$ bit does not affect the exponent and so the removal of a number of bits equal to the initial exponent value, say $q$, results in an exponent reduction of $q-1$ and a final exponent value of 1 . Removing any other bits would force the exponent to 0 which is reserved for special cases and may not be used. The bit selection for Case 2 is shown in Figure 5.24b. 


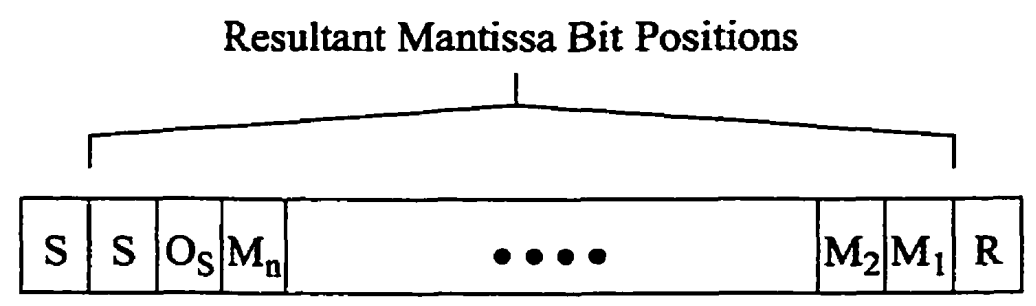

a) Case 1: One sign extension bit

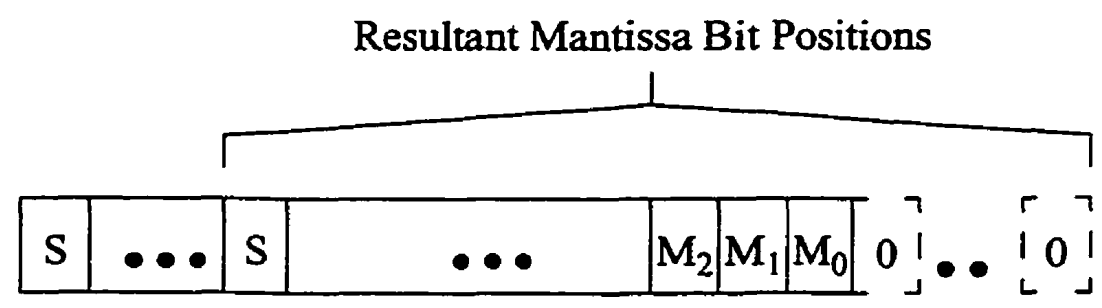

b) Case 2: More than two sign extension bits, Exp $>1$

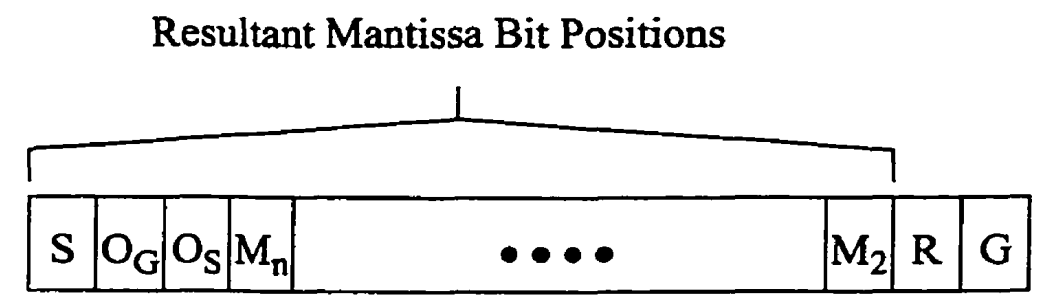

c) Case 3: No sign extension bits

\section{FIGURE 5.24. Selection of Mantissa Result Bits - Version II}

A special case exists, Case $2 b$, when there are two or more sign extension bits but the working exponent is only one. This limits the removal of the sign extension bits to only one and causes rounding to be necessary, since the trailing bits are no longer 0s. As only one of the two extra bits added for overflow is removed, the second bit remains as a significant digit for rounding. The LSB of the mantissa becomes the $\mathrm{R}$ bit. Case $2 \mathrm{~b}$ mimics the behavior of case 1 and is treated the same. 
The final case, Case 3, occurs if there are no sign extension bits. This indicates that the mantissa has undergone a rotational growth by an order of magnitude. This condition is dealt with the same way as in version I of the postprocessor. A rounding decision is based on the two LSBs of the input mantissa and the exponent must be increased. Case 3 is shown in Figure 5.24c.

\section{Normalization Control}

The determination of any of the above cases is performed by the State Logic block using the working exponent and the count of the sign extended bits. The sign extension bit counter shown in Figure 5.26 is a leading zero counter whose input is the mantissa with the sign bit removed. The sign bit serves to invert the mantissa bits before they enter the counter. In this way the leading zero counter can count the number of sign bits whether they are 0 s or $1 \mathrm{~s}$. The leading zero counter is referred to as leading zero anticipatory logic [40] and uses a tree structure based on 4-bit blocks to primely encode the number of leading zeros. Figure 5.25 shows an example of a 16 bit leading zero counter along with the output signal generation. The signals $T_{n}$ serve to indicate whether or not the block in question contained the threshold between zero and non-zero bits. The sum of leading zeros at each stage is composed of the bits $S_{n}$, where $n$ is the bit position and $L Z$ is the final leading zero count. If the input was all zeros then the MSB of the result is forced to 1 and the remaining bits set to 0 . If the most significant sub-block in a larger block asserts the $T_{n}$ signal then its count is propagated to the output. Otherwise, the count of the least significant sub-block is passed as the output count. To build larger counters, the 16 bit block is duplicated and the output stage expanded by one bit to form a 32 bit counter. A 32 bit 
counter is the largest required in the implementations in this work but larger counters can be produced in the same manner.

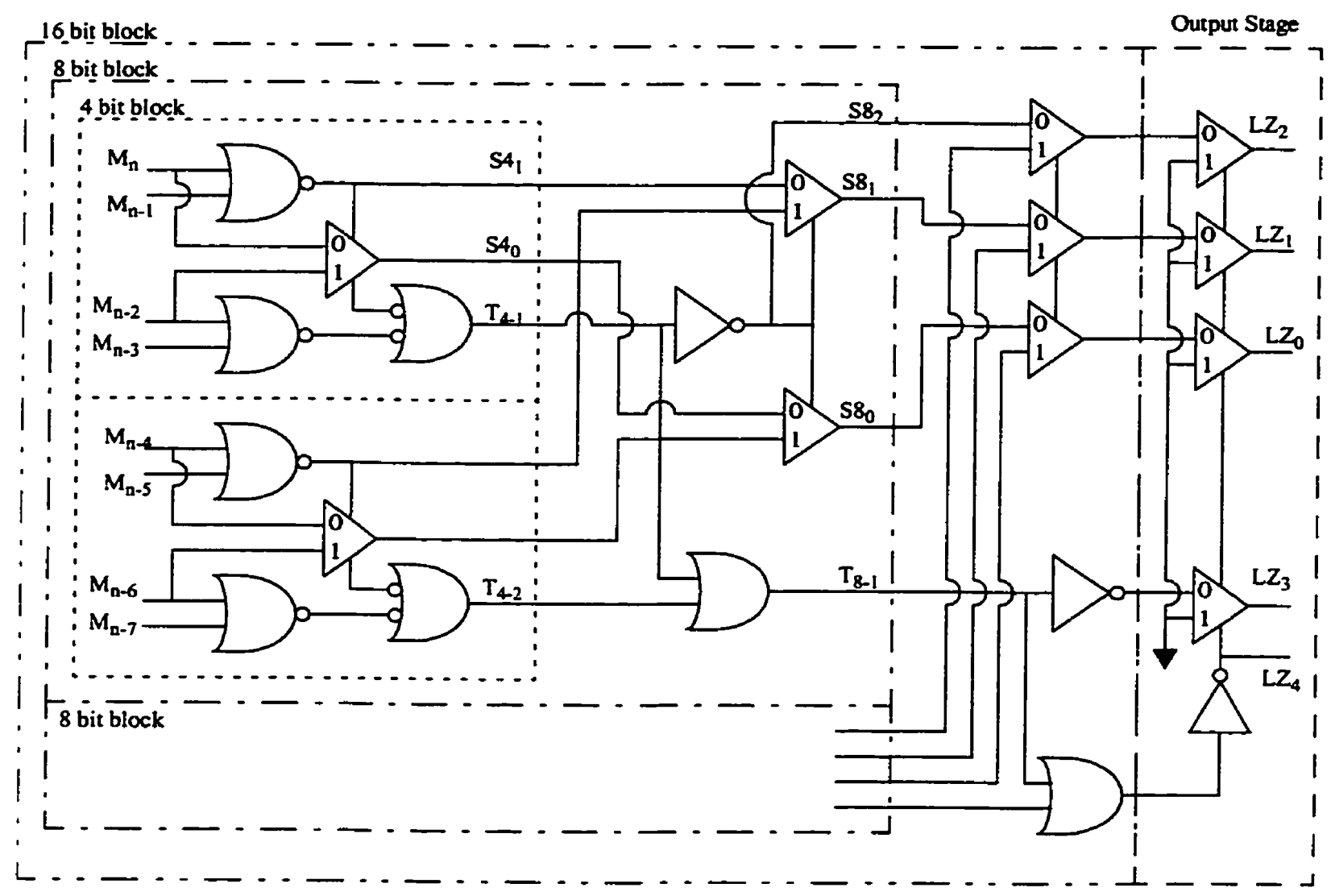

FIGURE 5.25. 16 Bit Leading Zero Counter

The overall architecture for version $I I$ of the postprocessor is shown in Figure 5.26. The generation of the result mantissa has two possibilities. The first possibility occurs in Cases $1,2 \mathrm{~b}$ and 3 where all bits of the mantissa are known and can be put in the proper bit position with the bit selection already presented. In these cases however a rounding decision must be made and the mantissa possibly increased by 1 ulp. This is accomplished in an adder with the carry in bit being the additional 1 ulp. The architecture of this adder need not be a carry-select form to increase speed, as the postprocessor stage is not the critical 
timing stage that limits the clock speed. As in version I the rounding can lead to overflow. This is dealt with in the way previously described and the exponent is incremented accordingly.

The second possibility occurs in Case 2 where the mantissa must be shifted by a variable number of bits. This is accomplished in the shift fabric which is made up of $4: 1$ and 2:1 multiplexer stages. The amount of the shift is the number of sign extension bits to a maximum value equal to the input exponent as already discussed. The potential new exponent is calculated using the number of sign extended bits to see if it underflows or if it equals the reserved value of 0 . If this is the case, then the maximum shift allowed is the value of the working exponent. A multiplexer is used to provide the proper shift amount, either the sign count or the working exponent, to the shift fabric.

The update of the exponent is performed using the result of the mantissa overflow detection, the decoded input data state and the sign extension bit count. The sign count is subtracted from the input exponent using a 2 's complement adder. As the possible exponent increment due to mantissa overflow occupies the carry in position of the adder, the increment of one due to the 2's complement of the sign extension count must be included in with the operand inputs to the adder. A second increment must be performed to account for the scaling factor. Recall that the overflow bit allowed for an order of magnitude increase may be removed with no change to the exponent. If six sign extension bits are to be removed then the change to the exponent is minus five. The addition is accomplished using sum and carry vectors obtained by performing 3:2 compression as previously described. The three terms, the working exponent (WE), the l's complement of the sign 


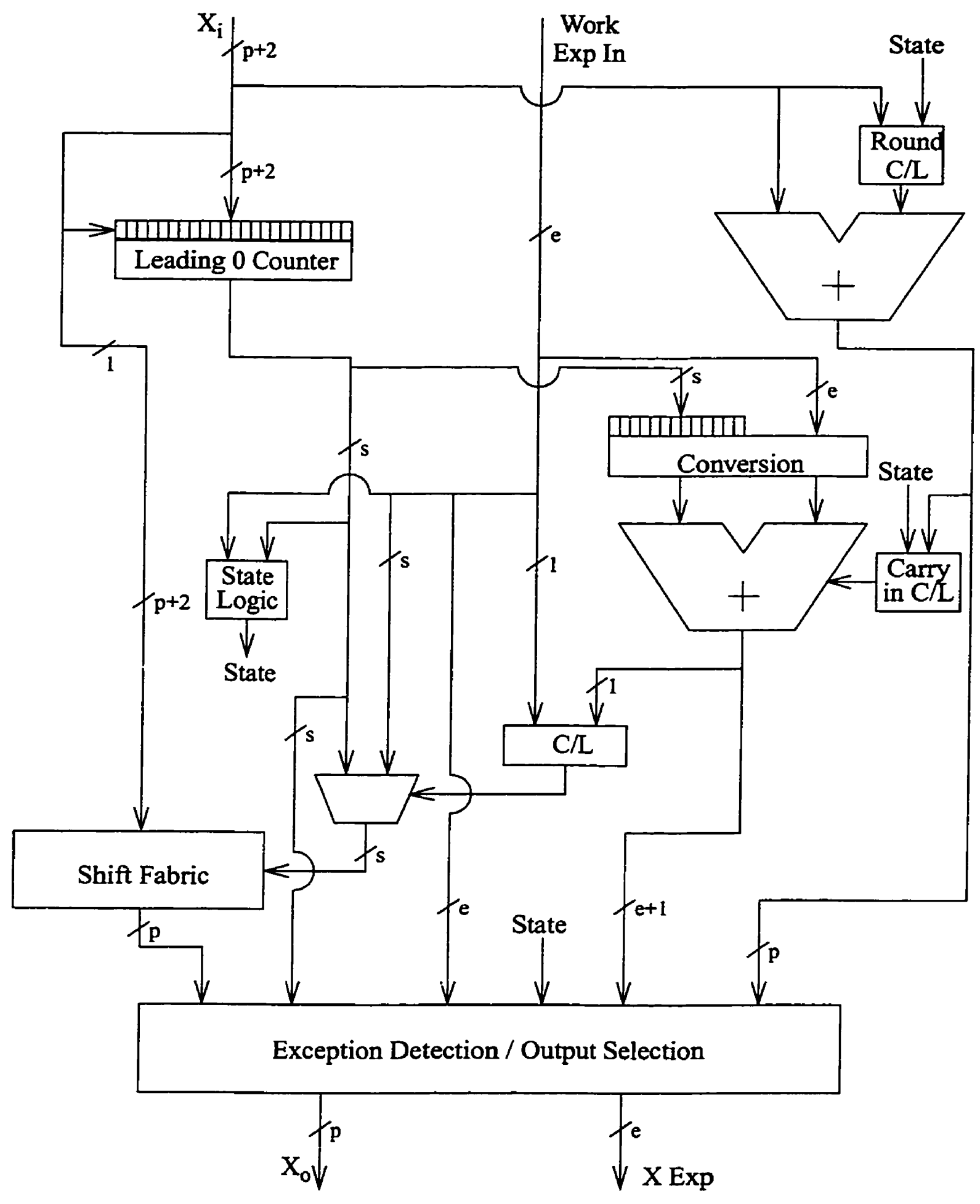

FIGURE 5.26. Post Processor with Full Normalization (X Data Path) 
count $(\overline{S C})$ and the two additional ulp for the 2 's complement and the $O_{G}$ bit removal, are converted to sum $(S)$ and carry $(C)$ vectors using one level of logic gates each.

\begin{tabular}{|c|c|c|c|c|c|c|c|}
\hline $\mathrm{WE}_{7}$ & $\mathrm{WE}_{6}$ & $\mathrm{WE}_{5}$ & $\mathrm{WE}_{4}$ & $\mathrm{WE}_{3}$ & $\mathrm{WE}_{2}$ & $\mathrm{WE}_{1}$ & $\mathrm{WE}_{0}$ \\
\hline$\overline{\mathrm{SC}}_{7}$ & $\overline{\mathrm{SC}}_{6}$ & $\overline{\mathrm{SC}}_{5}$ & $\overline{\mathrm{SC}}_{4}$ & $\overline{\mathrm{SC}}_{3}$ & $\overline{\mathrm{SC}}_{2}$ & $\overline{\mathrm{SC}}_{1}$ & $\overline{\mathrm{SC}}_{0}$ \\
\hline 0 & 0 & 0 & 0 & 0 & 0 & 1 & 0 \\
\hline
\end{tabular}

a) Input data for exponent evaluation

\begin{tabular}{|l|l|l|l|l|l|l|l|l|}
\hline $\mathrm{C}_{7}$ & $\mathrm{C}_{6}$ & $\mathrm{C}_{5}$ & $\mathrm{C}_{4}$ & $\mathrm{C}_{3}$ & $\mathrm{C}_{2}$ & $\mathrm{C}_{1}$ & $\mathrm{C}_{0}$ & 0 \\
\hline 0 & $\mathrm{~S}_{7}$ & $\mathrm{~S}_{6}$ & $\mathrm{~S}_{5}$ & $\mathrm{~S}_{4}$ & $\mathrm{~S}_{3}$ & $\mathrm{~S}_{2}$ & $\mathrm{~S}_{1}$ & $\mathrm{~S}_{0}$ \\
\hline
\end{tabular}

B) Input data after bit compression

\section{FIGURE 5.27. Data Presentation for Exponent Update}

If underflow has occurred in the result of the exponent update adder, then it is clear that the number of sign extension bits exceeds the allowable shift. In this case the maximum shift allowed is equal to the input exponent, however, a recomputation of the final exponent is not required. The resultant exponent in this case equals the working exponent minus the shift, plus 1 for the 'free' removal of the $O_{G}$ bit, which, of course, always equals 1. The exponent is, therefore, known and the output can be set without any further computation.

The final portion of the postprocessor is a control block. The Control block chooses the proper exponent and mantissa from the calculations performed and detects if overflow has occurred. In the case of overflow the exponent is forced to all $1 \mathrm{~s}$ while the 
mantissa is forced to all 0 s signifying a $+\infty$ result. Likewise a zero result forces both the mantissa and exponent bits to 1s. As before, underflow can not occur and is, therefore, not dealt with. 


\section{CHAPTER 6}

\section{BEHAVIOURAL SYNTHESIS}

Behavioural synthesis is an emerging computer aided design method which allows the design problem to be described in algorithmic terms and constrained by user defined requirement specifications resulting in an optimized realization at the structural level. The description is a higher level of abstraction than the typical register transfer level (RTL) description that stipulates the path of the data along with either behavioural or structural descriptions of what operations are performed with the data at each stage. In behavioural synthesis descriptions, the internal details of the datapath, any controllers which may be required, and other internal details that do not directly affect the input/output requirements are not specified. In the remainder of this chapter the concept of behavioural synthesis will be examined in more detail along with some of its advantages and disadvantages. The method used for this work, and an outline of the tool developed, will be outlined in the concluding section.

\subsection{Behavioural Synthesis Method}

Behavioural synthesis performs a highly optimized transformation from an algorithmic design to the RTL level. Many complex designs are written and tested at the algo- 
rithmic level to assure their correctness and accuracy. With traditional methods, decisions about the implementation details, such as the architecture required to implement the algorithm, had to be made and coded in RTL descriptions. If a designer wanted to test various architectures, then each one needs its own RTL description. This obviously requires considerable effort in order to explore a large design space. The process of behavioural synthesis allows for the automated exploration of the design space. The algorithmic description is constrained by a set of high level constraints, such as clock period, latency, throughput and resource count, and synthesized in order to produce different RTL versions

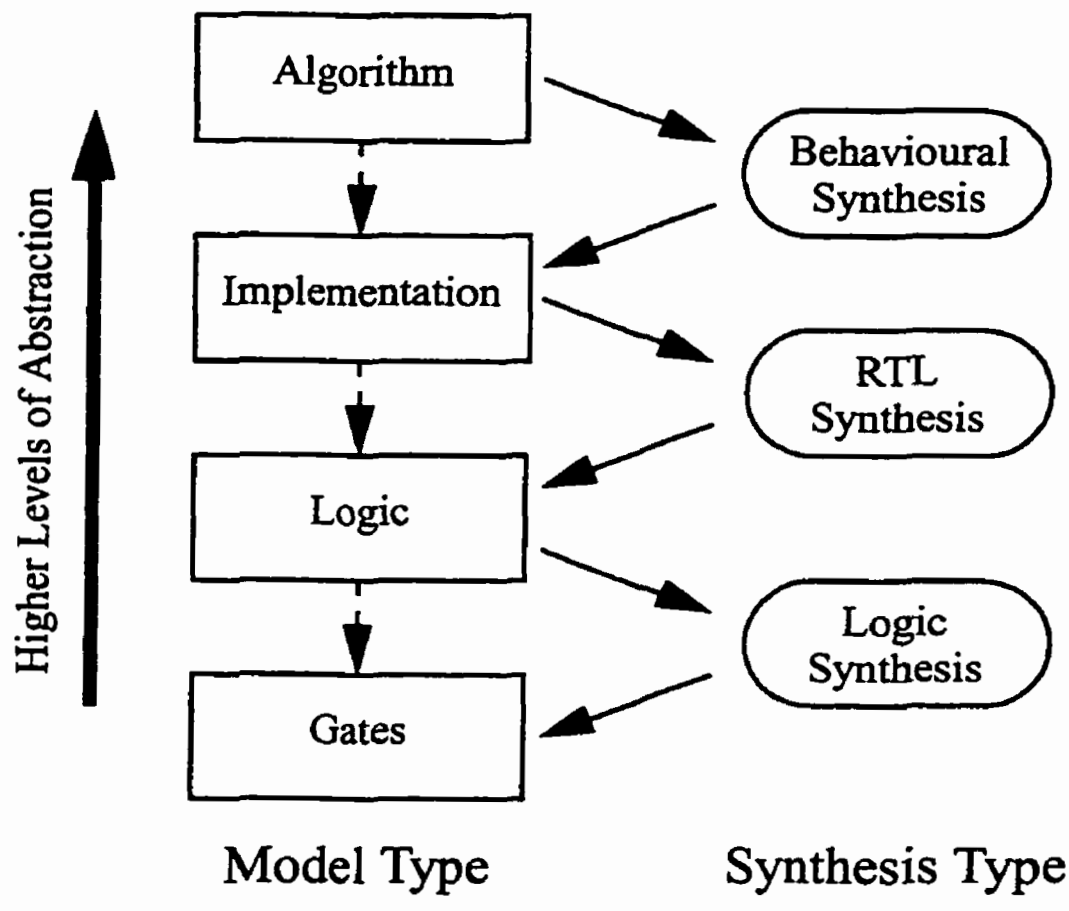

FIGURE 6.1. Model Abstraction Level and Synthesis Relationship

for evaluation. When the optimized solution has been found and chosen then the automated process continues with RTL and logic synthesis in the normal manner. The relation- 
ship between the level of abstraction of a description and the synthesis required is shown in Figure 6.1

Behavioural synthesis can be defined by its primary functions: scheduling, allocation and mapping. Scheduling is the task of assigning operations to control states. Only the times the inputs are available and the times the outputs are required are specified. Internally there is flexibility to schedule operations as needed while still assuring that the outputs are available when required. Allocation is the task of specifying what components are required to perform the necessary functions. The synthesis tool selects the number of arithmetic operators required, the number of registers required and the busses needed to tie them all together. Combined with scheduling, allocation provides a great deal of implementation flexibility. The number of components needed depends on whether it is possible to perform multiple operations with the same hardware and different control states or whether they must be performed concurrently. Mapping is the process of assigning arithmetic operators such as addition and multiplication to functional modules. If there are multiple operators of the same type and multiple functional modules then mapping assigns specific operators to specific modules.

The functional description of the design is written in a cycle-accurate specification. In cycle-accurate specifications, the Verilog always construct (VHDL process construct) is seen as a thread of control or, in other terms, a process. A specification can contain multiple always blocks, but each is seen as a separate, although communicating, datapath / finite state machine pair. A cycle-accurate description is an always block which specifies the timing relationships between reading the inputs and producing the outputs. These rela- 
tionships are specified by clock event specifications which are inserted between the procedural statements. The name cycle-accurate comes from the fact that the signals generated between two clock events, inside a single state, are only required to be accurate at the end of that state. The specification is flexible since only the input and output signals need to remain constant with respect to clock events. Statements which produce internal signals, those with no visibility outside the design, may be performed at anytime from when their operands are valid to when their result is required. These statements can be moved between states as required by the scheduler, in order to meet design constraints.

\subsection{Discussion of Behavioural Synthesis}

There are, of course, many benefits to working at this higher level of abstraction. The first advantage is a faster specification cycle as the descriptions contain fewer details and are more intuitive to produce. The second advantage is quicker verification and increased design reuse. Since the descriptions are more abstract than RTL descriptions the design simulates more quickly. The high level of abstraction also leads to easier reuse of design specifications. A third advantage is a more efficient architectural exploration. The ease with which different implementations can be generated by altering the high level constraints allows for faster and easier exploration. A final advantage is that the behavioural description style is clearer and shorter than RTL code, is easier to maintain as only one version is needed and it allows for easier changes later in the design cycle, if required.

There are, of course, designs that do not lend themselves well to the behavioural synthesis approach. The Synopsys ${ }^{\odot}$ Behavioural Compiler User Guide [41] states that 
designs which are not appropriate are those that are thought of as FSMs, those that have arbitrary 'goto' type state transitions, asynchronous designs, designs with random control and no datapath, designs with no need for resource or register optimization and those where latency and throughput trade-offs are not possible. The designs that are appropriate for behavioural synthesis are those with algorithmic computations, complex data flow and flexible latency and throughput. The synthesis manuals encourage a module by module decision for all parts of the design and a mixture of traditional RTL and behavioural specifications in the final system model.

An analysis of the strengths and weaknesses of the commercial behavioural synthesis tools reveals that they are most applicable to design modules that have a great deal of flexibility in their implementations. To generate the various implementation versions, the synthesis tool needs to be able to make trade-offs in order to meet the constraints. It was found that, for designs which lacked sufficient flexibility, the results of behavioural synthesis were not adequate. The CORDIC processor was found to be such a design. If given the high-level algorithm of rotation in cartesian coordinates, and none of the details about Volder's method, behavioural synthesis techniques would have yielded a workable solution. The problem is that the solution would suffer from the same drawbacks of multiplication and high memory requirements that motivated Volder's work. For detailed algorithms which are well developed and optimized for implementation there does not exist the flexibility that the process of behavioural synthesis requires. Consider the CORDIC process: there is little to no room for rescheduling operations as they are sequential in nature. Small adjustments could be made to the scheduling of the angle calculations as 
they are not on the critical path but this leads to little or no real benefit. Similarly, there is little possibility of component sharing without affecting the integrity of the algorithm as all the components are required for every iteration and are, therefore, always in use.

\subsection{CORDIC Behavioural Synthesis Tool}

The question then remains as to how the benefits of behavioural synthesis can be achieved without relying on the current commercial design tools? The answer is to develop a new tool which makes the benefits of behavioural synthesis open to the CORDIC process. The goals of fast implementation and architectural exploration of the design space for a versatile and powerful algorithm such as the CORDIC warrant the development of such a tool. Having ruled out automated architectural exploration as unnecessary for the already well developed CORDIC algorithm, it was necessary to determine how to best achieve the implementation variation that is desirable. The behavioural synthesis procedure outlined above requires certain constraints to guide the work and control the generation of the various implementations for the datapath and control modules. It is possible to achieve the same wide range of implementations in a tool for the CORDIC process if the architectural possibilities are made available to the designer.

The inputs of algorithm description, clock period, latency, throughput and resource count that are normally used for behavioural synthesis are not all required for this tool. The algorithm description is not required in so far as the CORDIC method is concerned, but information concerning the necessary inputs and outputs along with their width and the mode of operation is required. The trade-offs available with the clock period, latency 
and throughput result in various forms of datapath and pipeline. Instead of providing this information it is possible to allow designers to directly select the architecture which best suits their needs. The reason for such a change is the fact that, unlike some behavioural synthesis tools which are part of large software packages, the CORDIC tool would have no knowledge of the underlying technology. In order to make architectural decisions based on latency, throughput and clock period, knowledge of the technology is vital. The tradeoffs and the achievable design space for a given set of architectures are technology dependent. It is impossible to say, for example, whether a particular architecture will meet the timing and area constraints of an application without detailed knowledge of the technology. In order to produce a tool that was flexible and able to adapt to newer technologies, it was decided to provide designers with some direct control over the architecture.

An analysis of the information required to produce the different versions of the CORDIC PE was conducted. This analysis led to the development of the input set which the tool would require from the designer. The input set is comprised of the input/output data width, the mode of operation, the angle input format, the angle output format, the desired version of preprocessor, the desired version of postprocessor, whether the pipelining is in full parallel or serial/parallel form and finally, if a serial/parallel pipeline is used, what degree of parallelism is desired. The input/output widths were restricted to the four possibilities presented in Chapter 4, while the mode can be either rotation or vectoring. With respect to the input angle input format, the possibilities are to have none, an angle set $\sigma$, or an angle value $z$. Applications require different angle inputs based on their function and mode of operation. A vectoring PE need not have an input angle while a rotation PE 
could use an angle set representation to allow increased parallelism with other PEs. The output angle format has the same three choices, none, angle set $\sigma$, or angle value $z$. In rotation mode the output angle is not relevant and may, therefore, be eliminated. The angle set representation may be desired for PEs in vectoring mode which are a master PE to others in the rotation mode. This situation arises in matrix mathematical applications where a set of vectors are rotated by an angle determined by the first vector. The choice of versions for the pre and post processor are restricted to the two versions of each which were developed. These versions were presented in Chapter 5 and allow for the optional activity scaling in the preprocessor and the optional normalization of the output mantissas for the postprocessor.

The final items of the input set deal with the pipeline architecture. The designer may require a full parallel implementation in order to achieve high throughput or may require a serial/parallel implementation to reduce the area required. If a serial/parallel pipeline is required there is still the question of what is the degree of parallelism required. In order to provide options in this area, the CORDIC iterations required for each of the various bit widths were examined to find suitable mappings to the architectures presented in Chapter 5. The lower limit was three iterations per stage based on the fact that a mapping of one corresponds to the full parallel implementation and a mapping of two would not result in savings in area suitable for most applications as was discussed in Chapter 5. The second limitation on the selection of parallelism was the mapping of the iteration types, either double or single shift, to the architectures. A poor selection of parallelism results in reduced performance and sub-optimal implementations as there are either dou- 
ble shift stages performing single shift iterations at half the possible rate, or there are wasted iterations at the end of the required iterations where the completed results are waiting to advance to the postprocessor. It was found that, for the 12 bit mantissa set of parameters, mappings of three or four iterations per stage were best suited while, for the remaining mantissa sizes, mappings of three or five iterations per stage were best suited.

With the input set of requirements, the tool must then be able to make the corresponding choices for the datapath and control portions of the architecture. The tool needs to have knowledge of the architectural possibilities and of how to map the required iterations, and their associated parameter sets, to the those architectures. The selection of, and mapping to, the architectures can be done with a set of decisions based on the input values. If certain architectures are needed, for example a $z$ datapath, the tool needs to implement those aspects of the CORDIC algorithm. The effect of the input parameter set on all aspects of the algorithm was examined and the design requirements of all possible combinations had to be considered by the tool. The number of unique CORDIC PEs that are required, based on the input set, and which had to be implemented in the tool, totalled 576.

The tool is written in the $\mathrm{C}++$ language and uses a graphical user interface (GUI) as shown in Figure 6.2. The input set options for synthesis are entered with radio style buttons and the output design directory is entered in a text area. When the choices have been made, the tool verifies that all options are selected and that the choices are valid. Once a valid set of choices has been entered the tool produces the RTL level Verilog ${ }^{\circledR}$ HDL code for the desired implementation. 


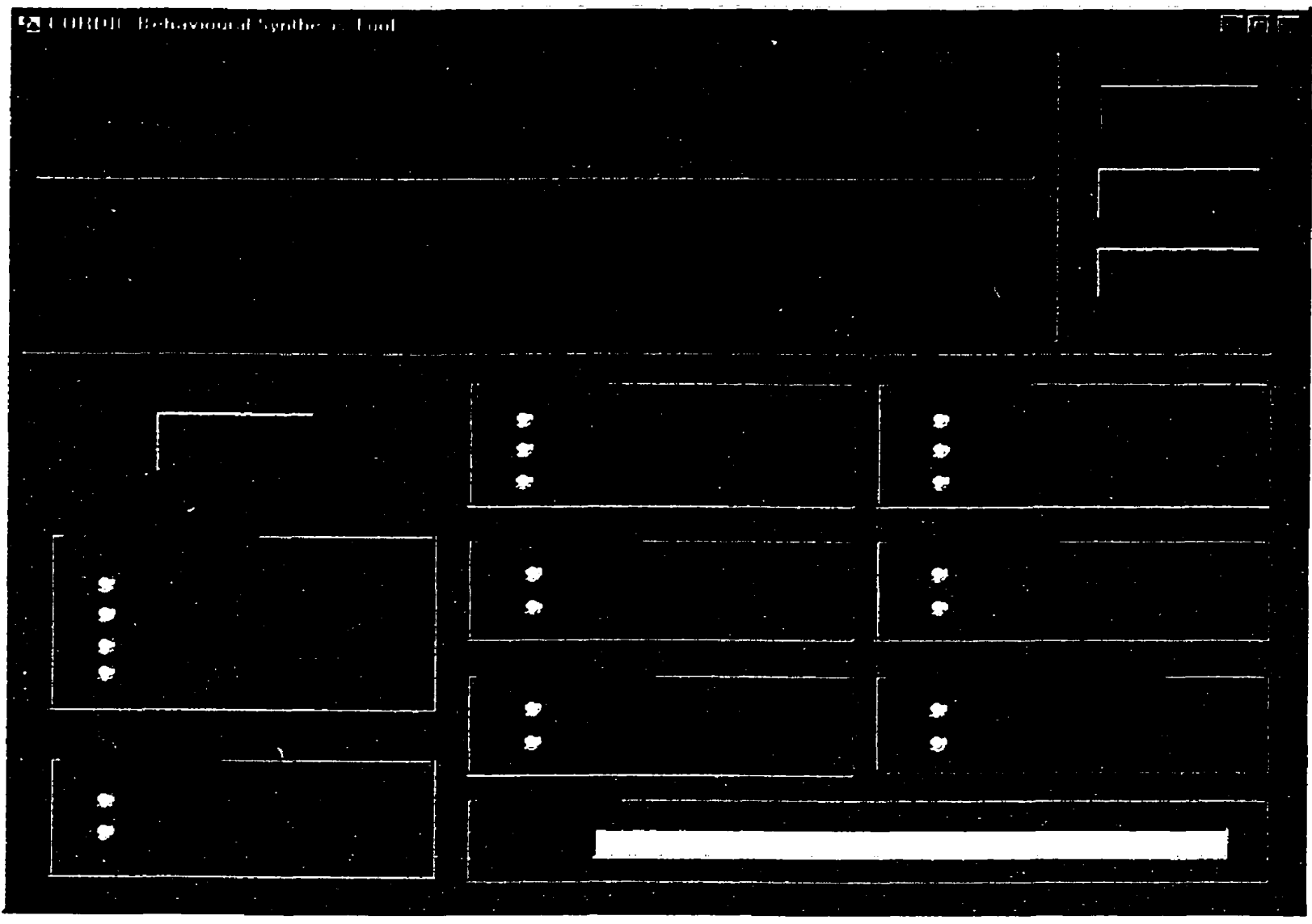

FIGURE 6.2. CORDIC Behavioural Synthesis Tool GUI

The CORDIC behavioural synthesis tool allows for the quick generation of different implementation level RTL design descriptions. The designer can then use the produced RTL design to conduct RTL synthesis trials, with their design constraints, to determine the best implementation for the required application. In order to speed up the exploration process, the designer can make use of the characterization data provided in Chapter 7 for a $0.35 \mu$ CMOS process from the Taiwan Semiconductor Manufacturing Company (TSMC), to narrow the field of implementations to trial. 
An examination of the RTL code produced by the tool will show that it is optimized in terms of architecture but not in terms of implementation. The next steps in the synthesis process are RTL and logic synthesis as shown in Figure 6.1. The tool relies on these later steps, and tools such as Synopsys ${ }^{\odot}$ Design Compiler, to perform the logic and RTL optimizations that are required. The case of the leading zero counter in the postprocessor Version I (section 5.3.1) will be examined to illustrate this dependence. The logic for the counter is built up from four bit sections to the largest version required, a 32 bit section. None of the implementations have 32 bit mantissas and so the RTL code pads the least significant end of the mantissa with sufficient ' 1 ' bits to produce a total of 32 bits. When logic optimizations are performed, as part of the logic synthesis step, the logic gates affected by the inputs driven by the constant ' 1 ' signals will be removed. It is possible for the tool to produce a different version of the counter for all anticipated bit widths but this is clearly not required. Having the tool produce general RTL code in this way makes the tool more versatile and allows it to be later expanded to produce other implementations of the CORDIC algorithm if required. Once produced, the RTL code is also better in this form, since it is well suited for design reuse, or easily modified, if required.

Another way that the architecture is optimized, and not the implementation, can be seen by examining the library modules which are used in the RTL description. The adders are a large part of the CORDIC design architecture and changes to their implementation has a great impact on the characteristics of the final design. Fast adders lead to a higher throughput but also occupy more area while smaller adders are slower. It is possible to use smaller but slower adders if they are not part of the critical data path. These are two exam- 
ples of implementation optimizations that are not made in the RTL code produced by the tool. The RTL code makes use of a library of built in functional modules from Synopsys ${ }^{\circledR}$ called the DesignWare Foundation Library. It contains parts, such as adders, which perform their required function but are not restricted to a particular implementation. The adder, for example, can be a ripple carry, carry look ahead, fast carry look ahead, BrentKung, conditional sum or ripple carry select adder as required to meet the constraints of the designer. The substitution of a particular adder is made at the RTL synthesis level to satisfy the synthesis constraints placed on the RTL level design code. This method of producing the code is better for both the tool and the produced code. The tool does not require knowledge of adders, or how to best produce them, making it more adaptable to new adder designs and the code produced can be used to explore a much larger design space.

\subsection{Behavioural Design Flow}

The design flow for the behavioural synthesis of CORDIC processors is shown in Figure 6.3. The flow takes the CORDIC from the algorithmic level to the implementation level as indicated in Figure 6.1 and starts, as it must, with the application requirements. Some aspects of the application may have firm requirements such as bitwidth, accuracy and permitted silicon area, which dictate certain architecture choices. Other aspects, such as latency and throughput, may have some flexibility, within firm upper and lower bounds, which provides a degree of choice in the possible architecture. Once the application architecture requirements are known, they are used to control the CORDIC Behavioural Synthesis Tool. If multiple versions of the CORDIC PE architecture will satisfy the requirements then it is possible to produce, and test, more than one RTL level design 
description in order to determine which is the optimal version. The behavioural synthesis tool is where the architecture requirements are transformed into an RTL description. The chosen RTL description is then used in commercial design tools, such as Synopsys ${ }^{\circledR}$ Design Compiler, in order to continue with RTL synthesis. It is at the RTL synthesis level that the design is constrained with application specific requirements, such as maximum area and minimum operating speed, in order to optimize the chosen implementation.

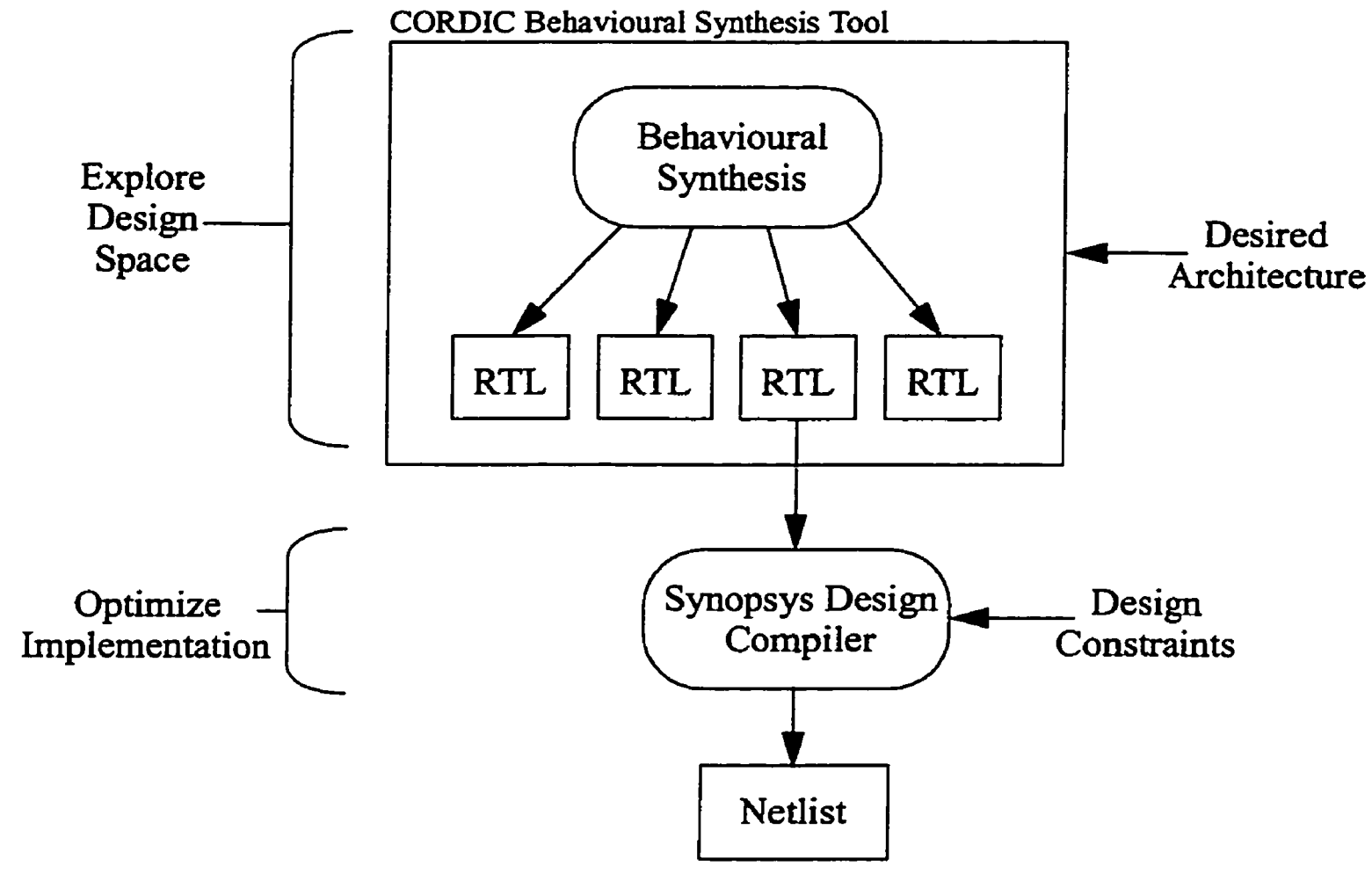

FIGURE 6.3. Behavioural Design Flow 


\subsection{Tool Operation}

When the designer has entered the required set of input parameters, the Generate Code button starts the behavioural synthesis process within the tool. Figure 6.4 shows the steps that are performed to produce the RTL level design description. The first action performed is a validation of the input parameter set. If a user has failed to make a choice in a field then an error message is displayed and the code generation process is halted. If all inputs have been selected then a validation check is performed to ensure that they are valid selections. Input selections such as an angle set $\mathbf{s}$ for the angle input in combination with vectoring mode, or no input angle for a $\mathrm{PE}$ in rotation mode, are detected. The target directory is also verified and created, if necessary at this stage of the process. In case of error

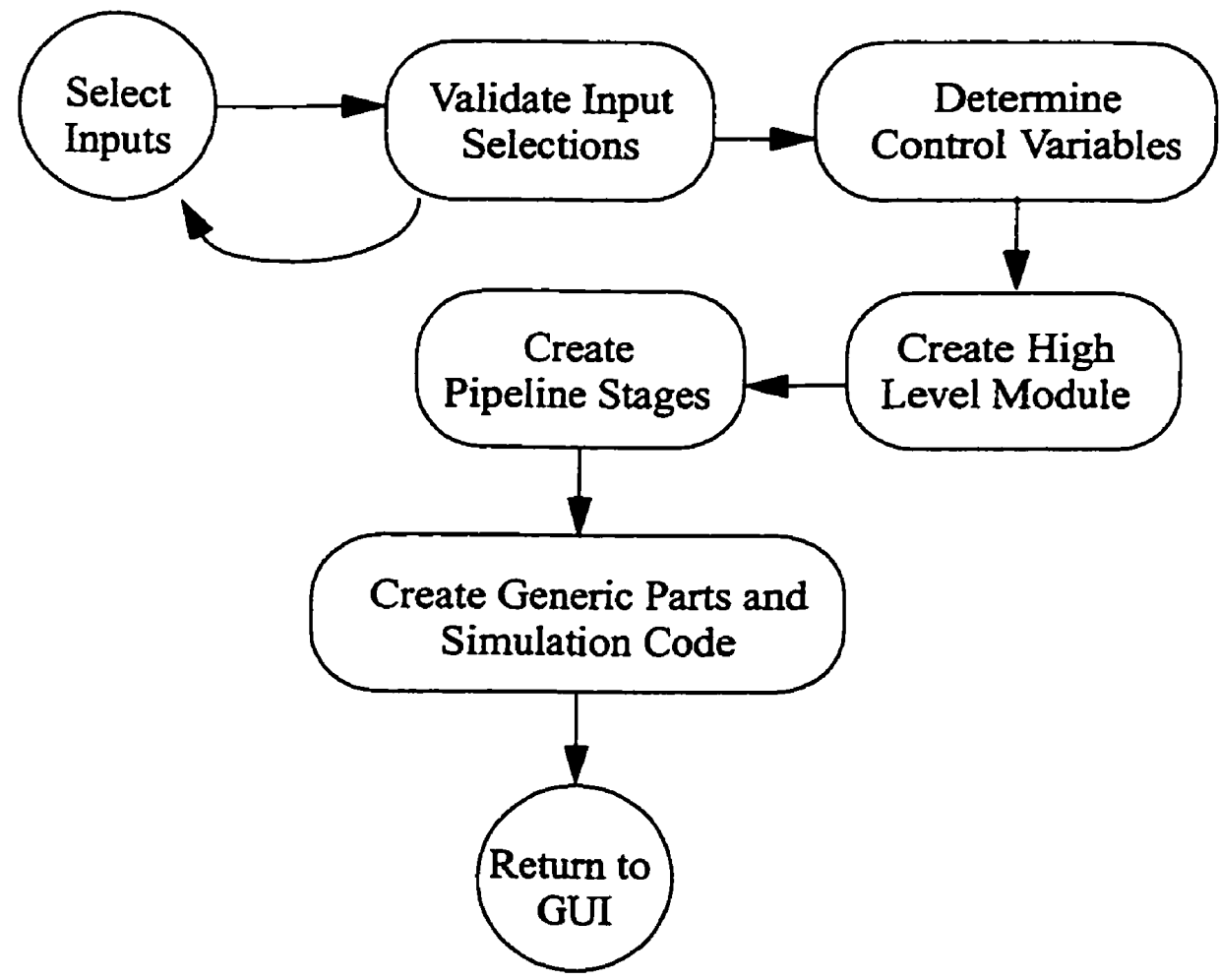

FIGURE 6.4. CORDIC Behavioural Synthesis Tool Operational Flow 
the process is halted until valid selections have been made. The next step in the process is the setting of the variables which control the code generation process. These are, for the most part, directly coupled with the input selections. An exception is the requirement for a $z$ datapath which depends on the choices made for the angle input and angle output format. In vectoring mode, a $z$ datapath is only required if the angle value output was chosen. If the angle set output format was chosen, or if no angle output is needed, then the running summation of the vector's angle need not be performed. In rotation mode, a $z$ datapath is not required if the input angle is in the angle set format and either no output angle, or an angle value output are required.

With the control variables set, the process of generating the desired RTL code can begin. The requirement for specific lines and sections of the HDL code are determined from the control variables in a distributed fashion. Throughout all stages of code generation, conditional statements are executed which ensure that both the proper portions of the CORDIC PE are generated but also that the parts interface properly. An example of this type of decision is the $z$ datapath. If not required then the portions of the code that deal with the $z$ datapath are not produced and the module interfaces are modified accordingly. In some cases, such as for the various bit widths, a conditional statement is used to produce parameters within the HDL code. Through the use of parameters whenever possible, much of the HDL code produced can be generic. This removes most of the dependence on the input parameter set, allows for more efficient coding of the synthesis tool and improves the HDL code by making post-generation analysis and modification easier. 
The code is written to a single file, called cordic pe.v, which is created in the desired destination directory. The first module to be written is the highest level module which is shown in Figure 5.1. The required preprocessor, pipeline stages, and postprocessor are instantiated along with the required pipeline registers and control signals. The modules which are written next are the pipeline stages for double and single shift iterations, the preprocessor and the postprocessor. The last section of code produced is generic and has no dependence on the input parameter set. In this section is the code required for the simulation of the Synopsys ${ }^{\odot}$ DesignWare Foundation Library parts. The code is required so that the modules instantiated from the synthesis library can perform their required functionality during simulation. During synthesis, the library modules are selected and the simulation code is ignored. When the code generation is finished a message is displayed to indicate a successful completion. A new set of parameters can be entered to produce a different version of the CORDIC PE or the tool can be closed.

\subsection{Technical Report}

A copy of the CORDIC Behavioural Tool program code along with a discussion on its features is contained in a seperate technical report [42]. This report also details issues concerning the optimization of the Verilog ${ }^{\circledR}$ HDL code produced by the tool with the commercial suite of Synopsys ${ }^{\circledR}$ tools. 


\section{CHAPTER 7}

\section{RESULTS}

In order to efficiently use the CORDIC Behavioural Synthesis Tool to explore the design space, as presented in section 6.4, designers need to know what variations of the PE are likely to fall within their design space. Designers are faced with decisions early in the design process dealing with, among other things, the power and area implications in selecting specific architectures and sub-blocks. Knowledge of what is achievable forms an important basis of the decision making process, as unachievable goals and choices made early in the design process will result in wasted time and effort. This chapter outlines the performance characteristics of various synthesized versions of the PE generated by the CORDIC Behavioural Synthesis Tool in order to provide a solid basis for the early decision making process.

\subsection{Characterization Environment}

It is important to discuss how the PEs were characterized prior to any discussion of the results. The results obtained are closely linked to both the process followed and the underlying technology. Changes in these factors mean changes in the design space which the designer can achieve. 
The technology used for this work was the TSMC $0.35 \mu$ Standard Cell Library available as part of the Canadian Microelectronics Corporation (CMC) Design Kit for Synopsys $^{\odot}$ and Cadence ${ }^{\odot}$ V4.1 [43]. The PEs were described using the Verilog Hardware Description Language (HDL). The HDL code was analyzed and sythesized using the Synopsys $^{(}{ }^{\circ}$ suite of digital design tools. Obviously a change in the minimum feature size will result in different operating characteristics but, perhaps less obviously, so will changes in the library used. Cells from different libraries with the same functionality may have different operating characteristics and physical properties. The synthesis results are likewise affected by the cells available within a given library. If a cell with a specific functionality is not available, then it must be replaced with two or more other cells in order to perform the same function. This will cause changes to the operating characteristics.

To obtain results for various versions of the $\mathrm{PE}$, it was decided to characterize the versions with mantissa bit widths of 12,16 and 24 bits. The version with a mantissa width of 18 bits was excluded due to the fact that its characteristics would be not that different from the 16 bit version. In addition, it is anticipated that this version will be the least used of the four as most applications studied used one of the other three bit widths.

The characterization data was obtained from versions of the PE which operated in vectoring mode. There is not a significant difference between the vectoring and rotation versions of the PE in terms of the metrics measured. The control signals are essentially the same for both and in each case one of the inputs is being driven to zero during the iterations, while the remaining two hold the computation results. 
Versions of the PE with and without the $z$ datapath were characterized. This variation represents a significant difference in both the area and power metrics due to the presence, or lack, of the architectural components that support the running sum of the angle computations. Versions of the PE using full parallel and serial/parallel pipelines were also characterized as the selection of the pipeline architecture has significant effects on the metrics.

The versions selected for characterization were synthesized three times using different constraints. These are referred to as Compile 1,2 and 3. In Compile 1, the aim was to determine the fastest possible implementation. To this end, each version was compiled using the same set of commands aimed to force the lowest possible delay on all register inputs and output signals. Constraining the synthesis in this manner has resulted in the fastest possible operation, but it also led to a waste in area and power. Signal paths not part of the critical path were optimized for speed along with all other signals. This was necessary in order to ensure that the fastest possible operation was accurately captured. In reality, the timing constraints for signals not on the critical path can be relaxed resulting in a reduction in area/power with no loss of speed.

In Compile 2, the aim was to determine the smallest possible implementation size. Each version was compiled using the same set of commands which constrained the maximum area of the design to zero. Synthesis possibilities that allowed for smaller designs without violating fan-in/out rules and transition times were chosen in order to ensure the smallest possible size. Constraining the design in this way led to small designs which suffered from slow operating speeds. 
In Compile 3, the aim was to produce synthesis results which were neither the fastest, nor the smallest, but instead offered the designer an idea of the area vs. speed tradeoffs that were possible. In general, when transitioning from a small to a fast design, the first gains in speed are made relatively cheaply in terms of area. At the other end of the continuum, the final gains in speed are usually only achievable with significant area costs. As this relationship is not linear, design constraints were sought that would limit the area to no more than $50 \%$ of the area used by Compile 1 , while resulting in significant speed improvements. It is worthy of note that the characterization metrics depend not only on the constraints themselves, but also on the synthesis process. A synthesis process that uses multiple incremental compiles to meet the constraints, vice a single compile, was found to produce poorer metrics. This is because the synthesis results from one compile are the starting point of the next and so previous decisions affect future ones. This situation can be avoided if the design is realistically constrained from the outset.

The $x$ and $y$ input data required to determine the energy metrics was generated in an auto-recursive fashion. The formulas governing the data generation were:

$$
\begin{aligned}
& x_{i}=r f^{*} x_{i-1}+n d_{1} \\
& y_{i}=r f^{*} y_{i-1}+n d_{2}
\end{aligned}
$$

where $r f$ is the recursive factor and $n d_{l}$ and $n d_{2}$ are normal distributions with zero mean and unit variance. Three sets of input data were generated with recursive factors of $0,0.5$ and 0.9 respectively to simulate the possible correlation between successive samples. 
There was no correlation imposed between the $x$ and $y$ inputs for the simulations as, most commonly, none exists in CORDIC systems.

In summary, 24 different versions of $\mathrm{PE}$ with constraints were characterized. Some versions differed in their HDL description and others were constrained differently in order to achieve results that spanned the design space. Table 7.1 details the possibilities available in each category. Versions of the PE corresponding to all possible choices were characterized and the measured metrics are presented in the remainder of this chapter.

The final sections of this chapter provide comparison metrics for versions using the activity scaled version of the preprocessor and the normalization enabled version of the postprocessor. The metrics were obtained for one of the versions in order to provide an idea of their impact on the overall metrics.

\begin{tabular}{|c|c|c|c|}
\hline $\begin{array}{c}\text { Mantissa } \\
\text { Bitwidth }\end{array}$ & $\begin{array}{c}\text { Z Data } \\
\text { Path }\end{array}$ & $\begin{array}{c}\text { Pipeline } \\
\text { Style }\end{array}$ & $\begin{array}{c}\text { Synthesis } \\
\text { Constraints }\end{array}$ \\
\hline \hline 12 & Yes & $\begin{array}{c}\text { Full } \\
\text { Parallel }\end{array}$ & Speed \\
\hline 16 & No & $\begin{array}{c}\text { Serial / } \\
\text { Parallel }\end{array}$ & Area \\
\hline 24 & & & $\begin{array}{c}\text { Speed and } \\
\text { Area }\end{array}$ \\
\hline
\end{tabular}

TABLE 7.1. PE Characterization Versions 


\subsection{Area}

The area of the compiled versions, measured in $\mu \mathrm{m}^{2}$, is shown in Figure 7.1 to Figure 7.4. It is important to note that the area measured excludes the interconnects. The area for the interconnects is not known until after placement and will vary depending on the number of metal layer available. More metal layers result in less routing congestion and an increased possibility that all routing can be done above the cells without adding more area.

The results show that a considerable amount of area can be saved using the serial parallel versions of the PE. The trade-offs for this saving will be examined in the following sections. The results also show that there exists an increased range in the achievable area for the larger bit widths. This is principally due to the larger adders and the increased number of pipeline stages which multiply the effect of any changes made. A comparison between the versions with, and without, a $z$ datapath reveals that there is an increasing benefit from its elimination as the bit width increases. It is also evident that the area savings are more for the full parallel implementations. These results are as expected since more architecture is eliminated in implementations with more pipeline stages and larger adders.

Of final note is the areas of Compile 3 for each of the versions. Recall that the target area was $50 \%$ or less of the largest compile. In most cases the results approached the $50 \%$ limit but not in all. In some compiles, notably the 24 bit version in Figure 7.1 , it was not possible to approach the limit in small increments. It was found that any changes in the constraints led to large increases in area. 


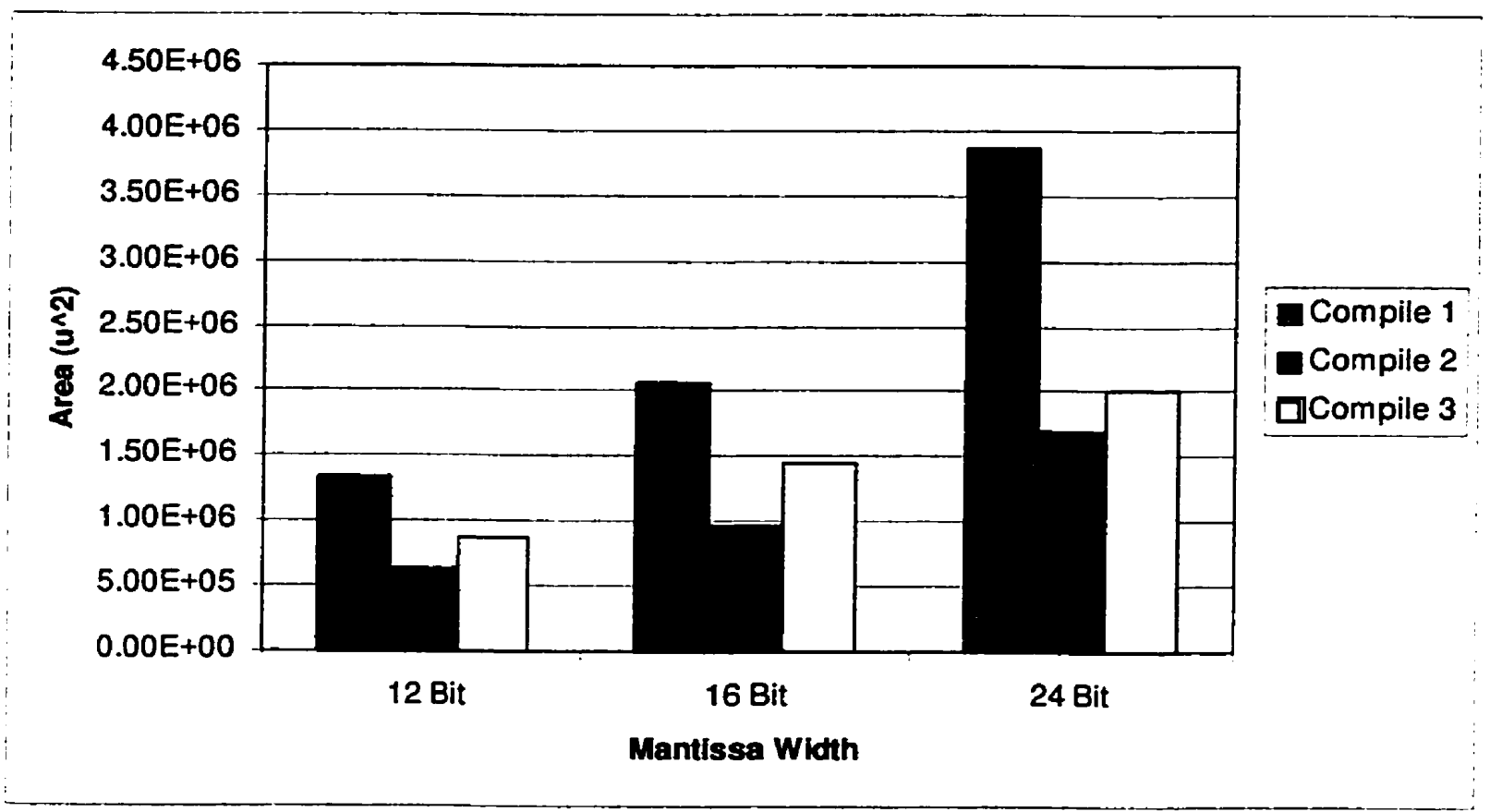

FIGURE 7.1. Area - No Z Path, Full Parallel

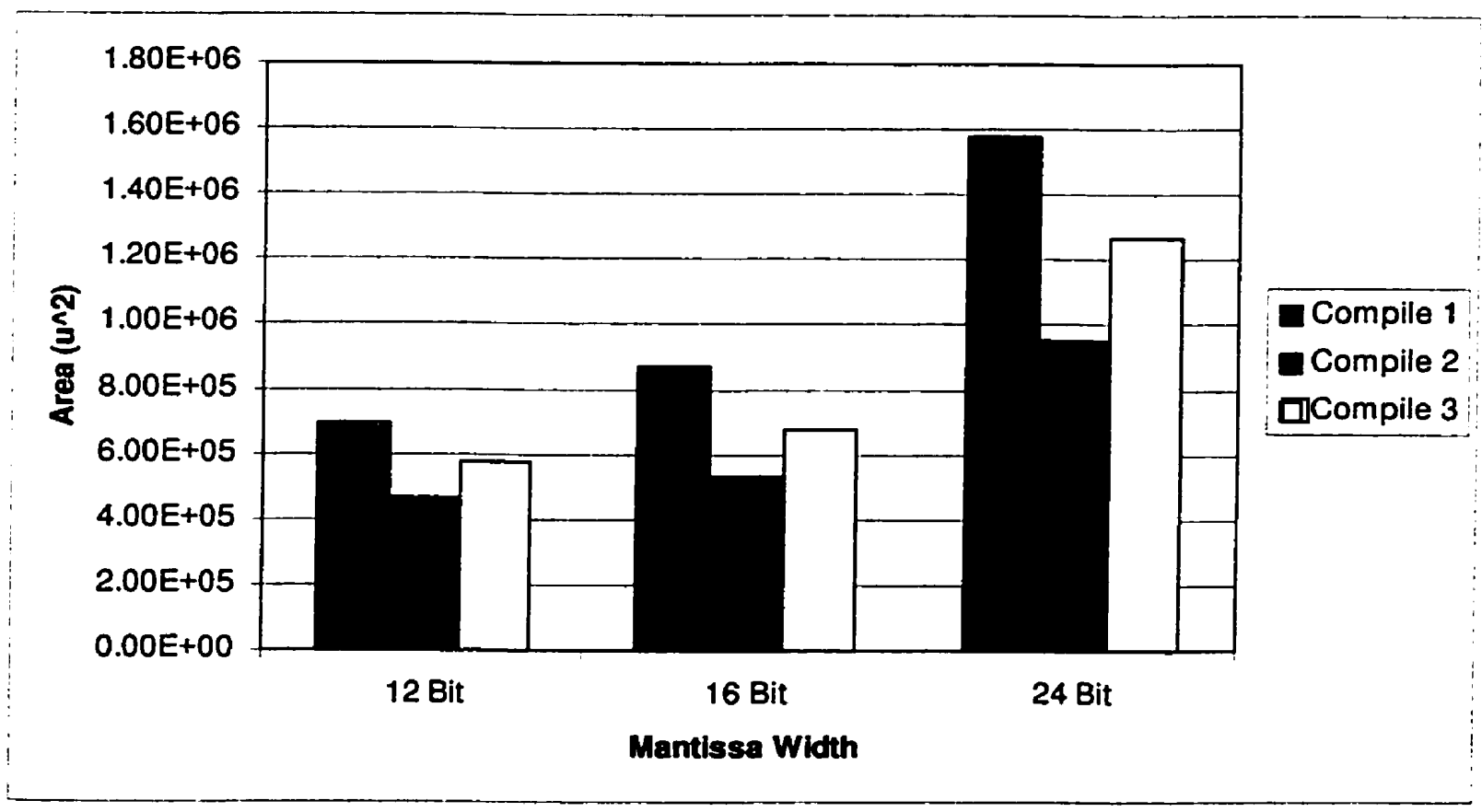

FIGURE 7.2. Area - No Z Path, Serial / Parallel 


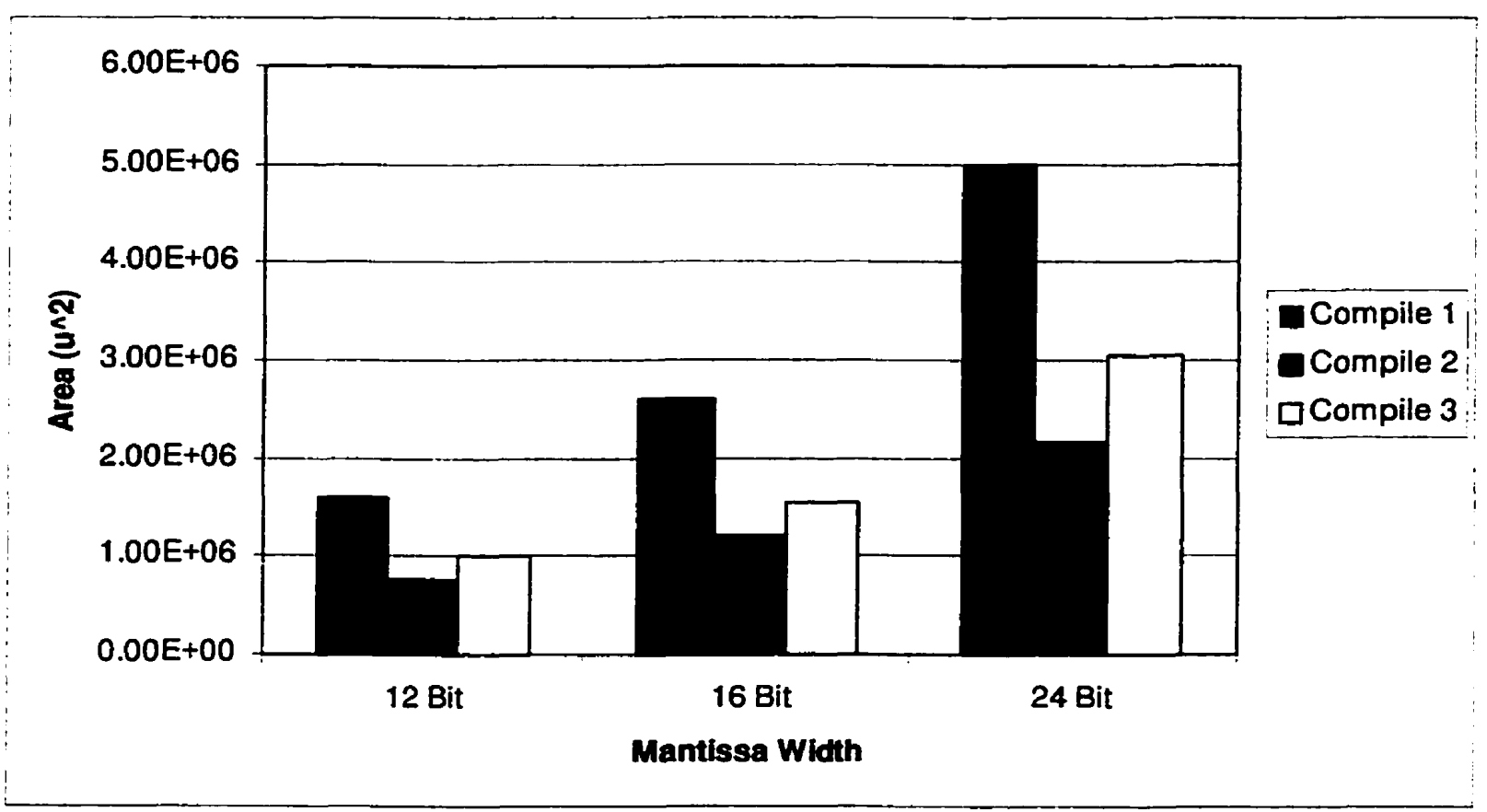

FIGURE 7.3. Area - Z Path, Full Parallel

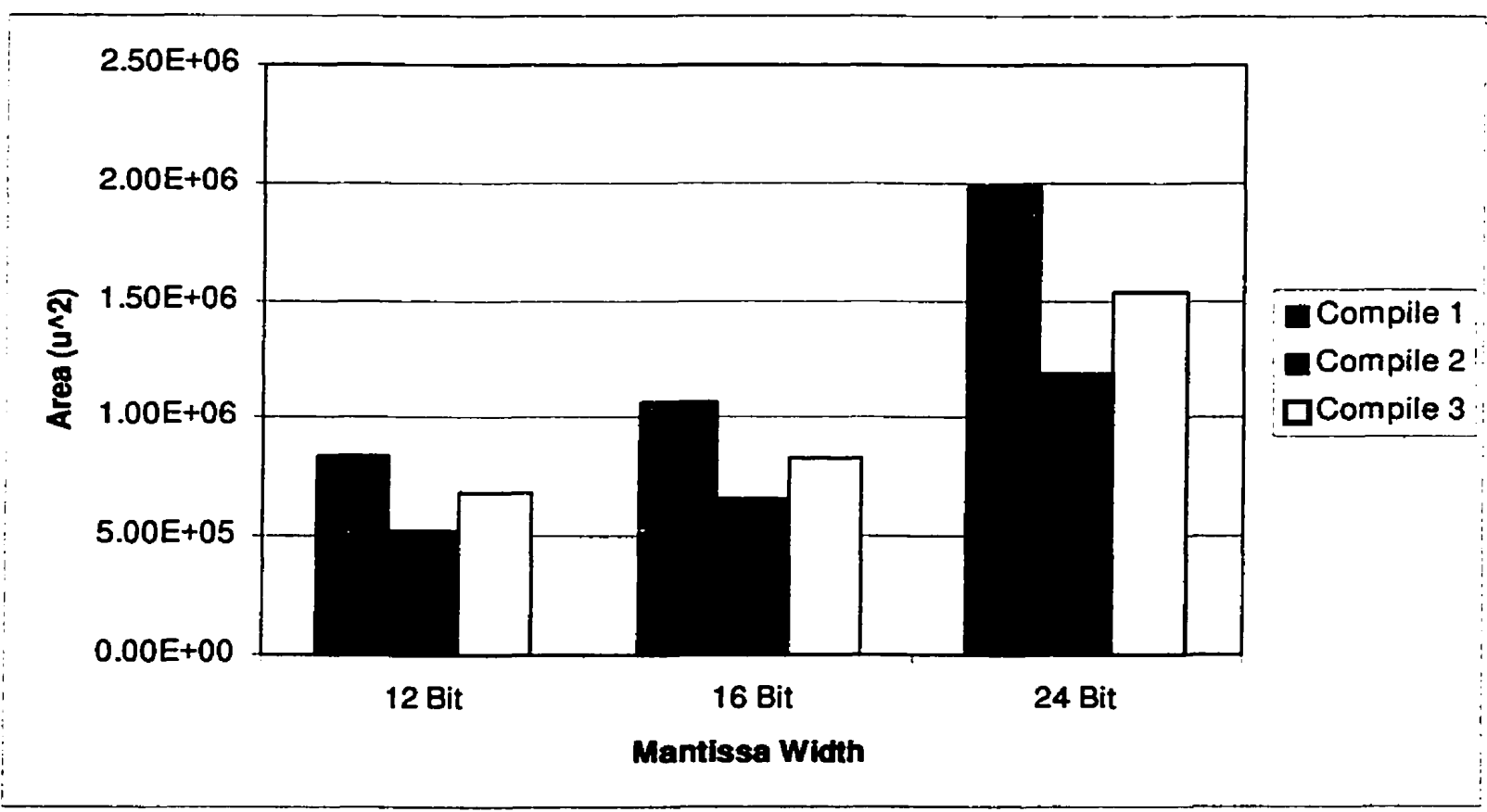

FIGURE 7.4. Area - Z Path, Serial / Parallel 


\subsection{Throughput}

Throughput is the measure of how many sets of input data are processed in one second. In the case of serial/parallel implementations the throughput is calculated with the system clock, and not the FSM clock, as it is the one that controls the movement of data into, and out of, the PE. The maximum throughput of the various versions of the PE is shown in Figure 7.5 to Figure 7.8 and is measured in samples per second. In all versions, the speed of operation, and hence the throughput, was determined by the computation time of the single shift pipeline stages which was only slightly longer than the double shift stages. This supports the decision to match the computation time of the stages for the two iteration types by performing two iterations in the single shift stages.

As expected, the inclusion, or exclusion, of the $z$ datapath did not have a significant impact on the maximum throughput. This is because the $z$ datapath computations, in all versions of the pipeline, take less time than those for the $x$ and $y$ datapath. The bit width had a small impact on the versions compiled for throughput and a larger impact on the versions compiled for area. This is because fast adders, namely conditional sum and carry select adders, only suffer a minimal increase in delay for a few additional bits due to their staged architecture. In the case of small adders, principally the ripple carry type adder, the impact of an increased bit width is a longer carry chain, increased delay and reduced throughput.

Analysis of the throughput metric in combination with the area metric reveals that, at this level, significant improvements in throughput can be achieved with small increases in area. This is possible, since there are ways to increase speed and throughput, such as 
using cells with increased drive strength and more advanced adder structures, without using the fastest, and largest, adders available.

It is clear from the results that there is a significant penalty incurred with the serial/ parallel versions of the PE. This is one of the trade-offs for the reduced area achievable with the serial/parallel versions.

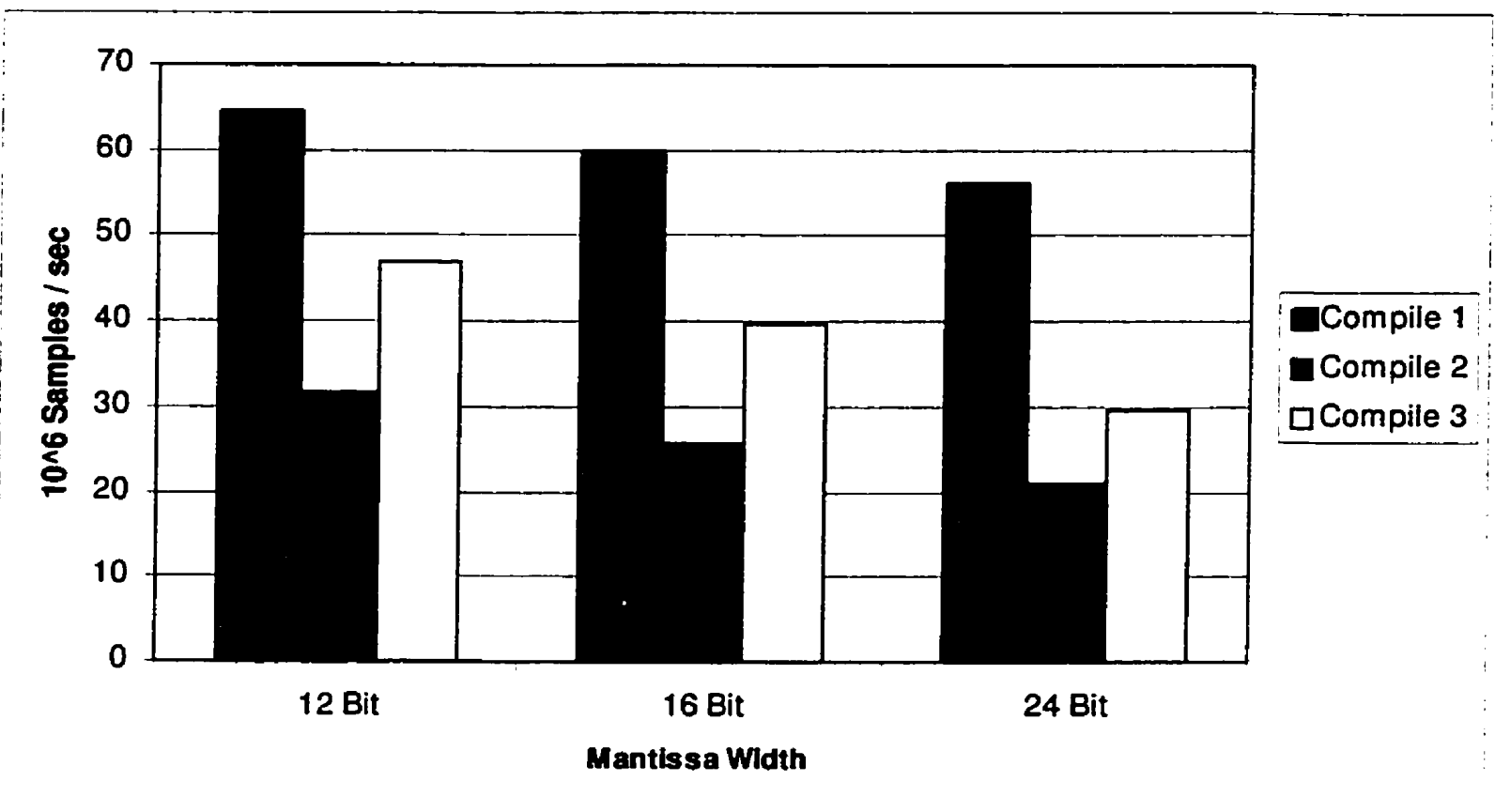

FIGURE 7.5. Throughput, No Z Path, Full Parallel 


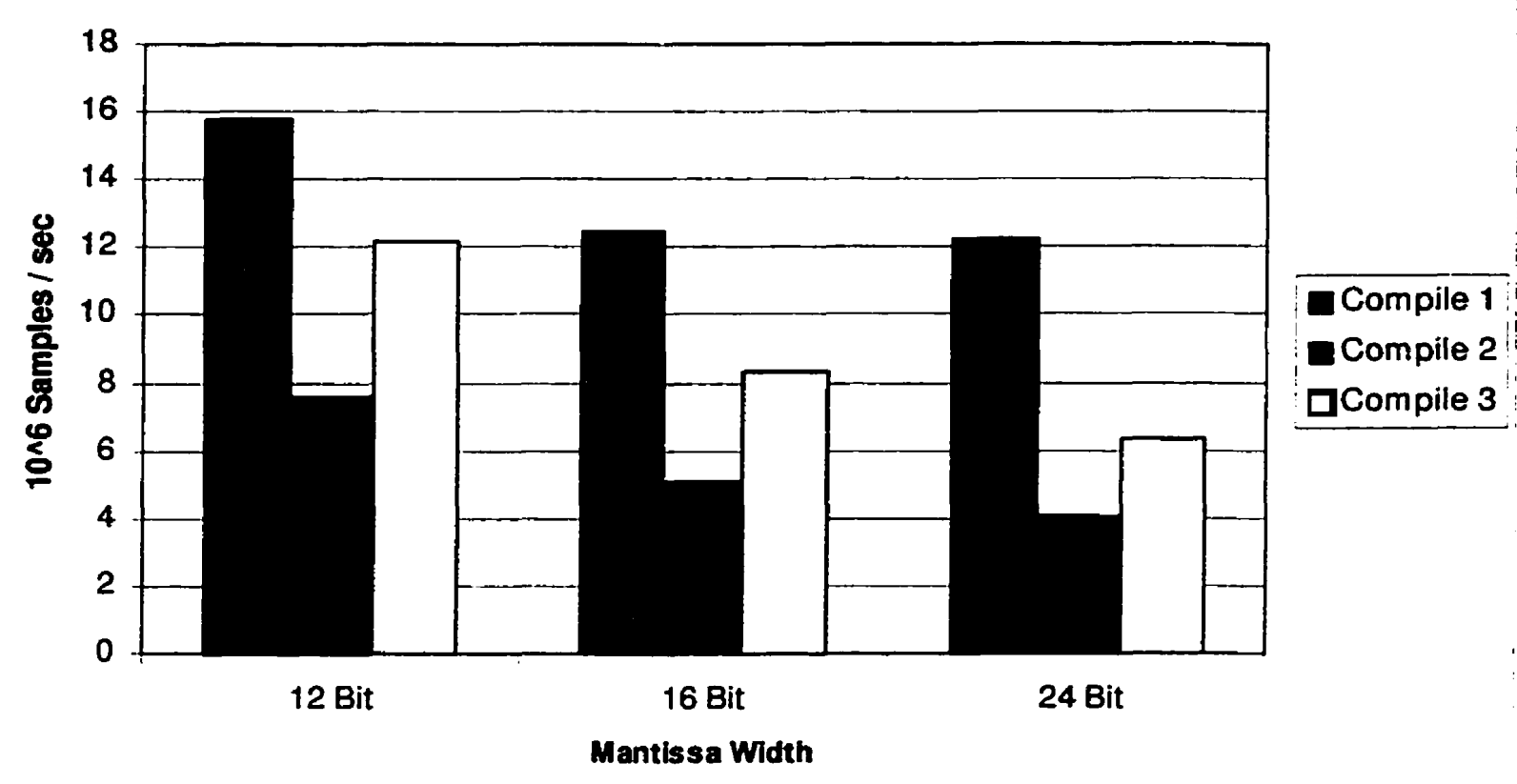

FIGURE 7.6. Throughput, No Z Path, Serial / Parallel

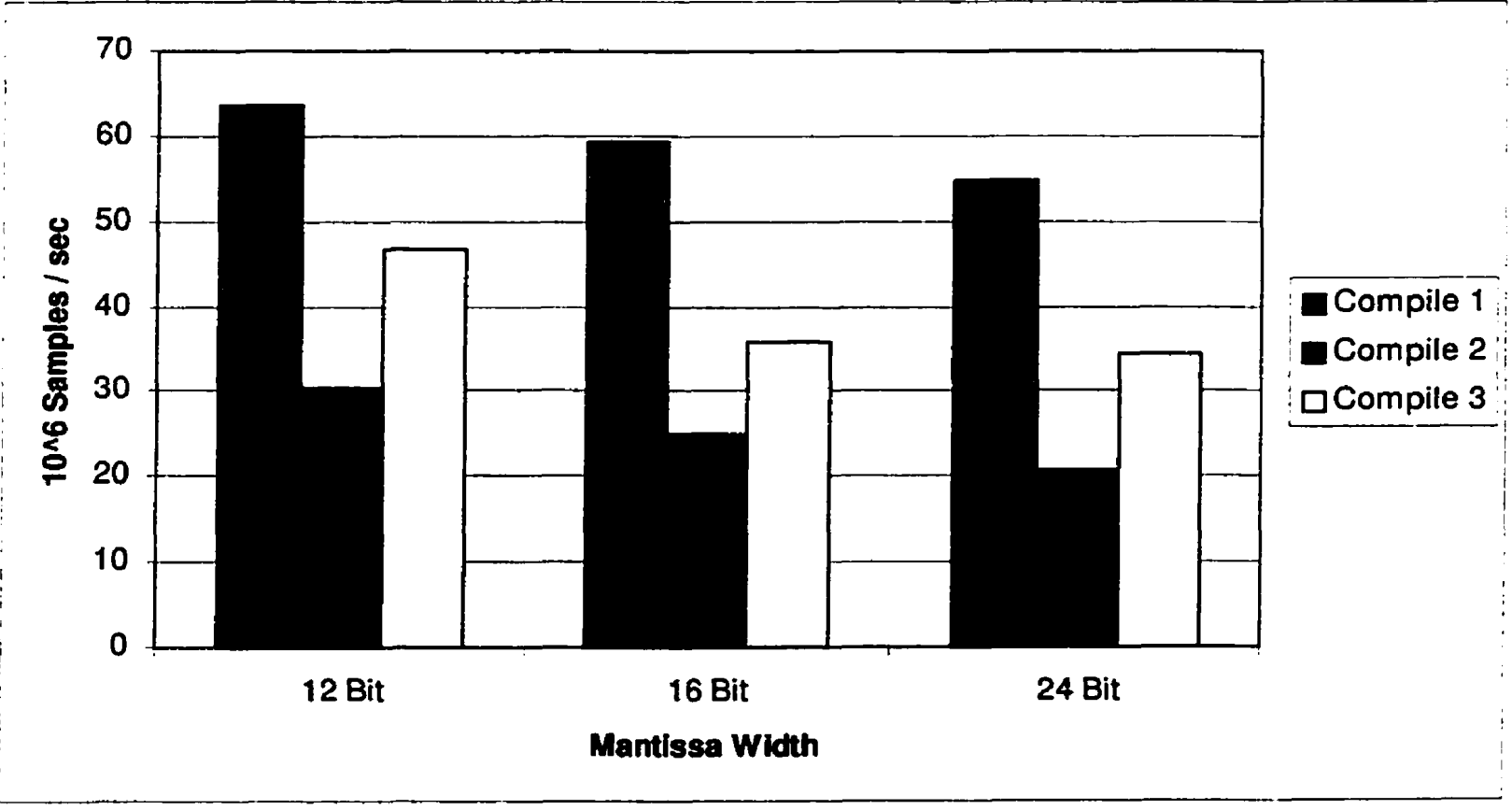

FIGURE 7.7. Throughput, $Z$ Path, Full Parallel 


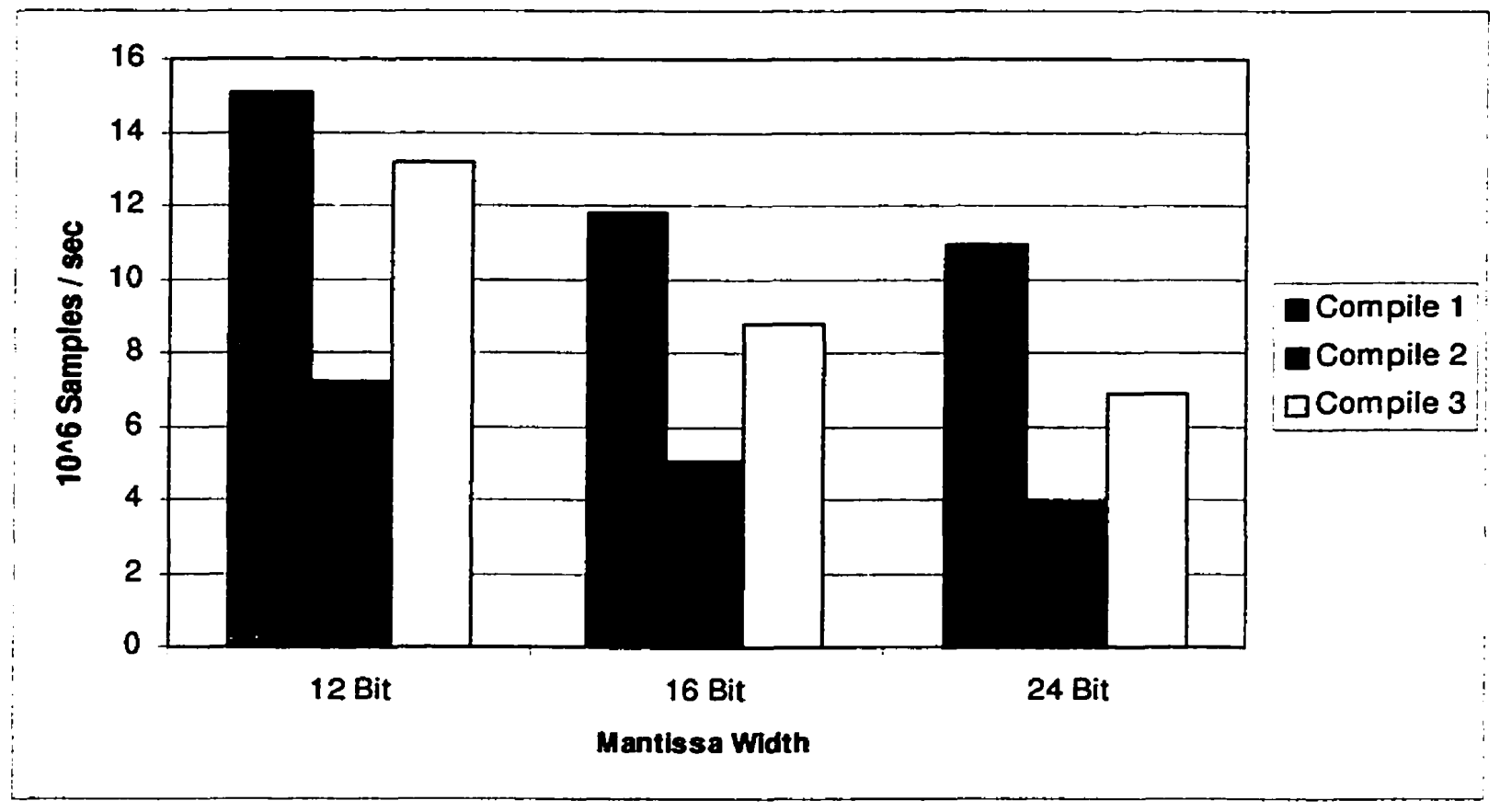

FIGURE 7.8. Throughput, Z Path, Serial / Parallel

\subsection{Latency}

The minimum latency of the various versions of the CORDIC PE is shown in Figure 7.9 to Figure 7.12 and is measured in nanoseconds. The latency is the time taken for the results to be presented at the output after the inputs are sampled and is a direct function of both the number of iterations/stages required and the fastest permissible clock speed.

The latencies for the serial/parallel versions are higher than those of the full parallel versions due to the mapping constraints of the pipeline stages as the number of double and single shift iterations do not map to the stages in an ideal manner. This is because the product of the pipeline stages and the iterations per stage are not necessarily equal to the number of iterations required. In these cases, extra iterations must be performed which do 
not affect the final results, but allow the proper dataflow through the stages. This increase in latency is a cost of achieving the area reduction with the serial/parallel implementations.

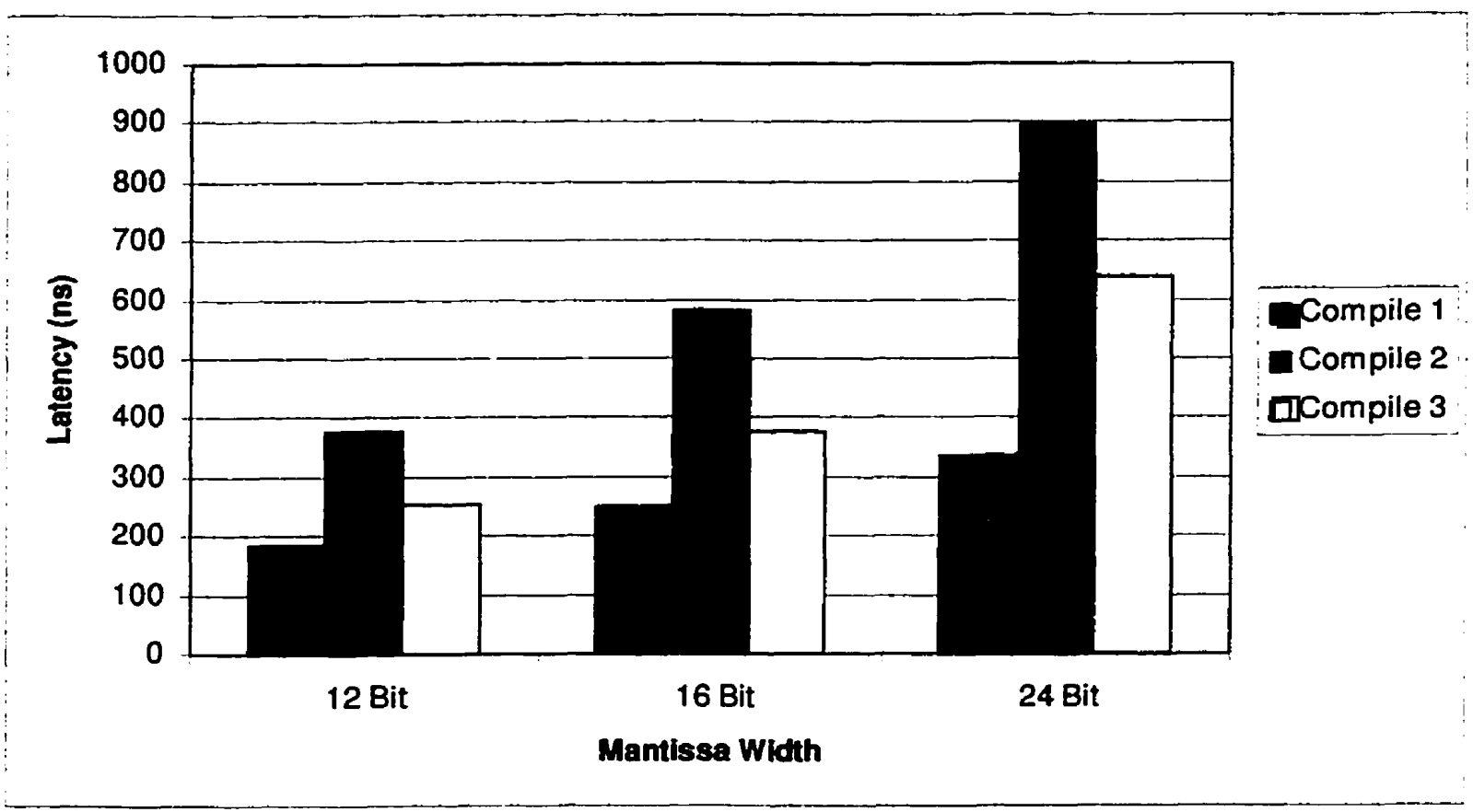

FIGURE 7.9. Latency - No Z Path, Full Parallel 


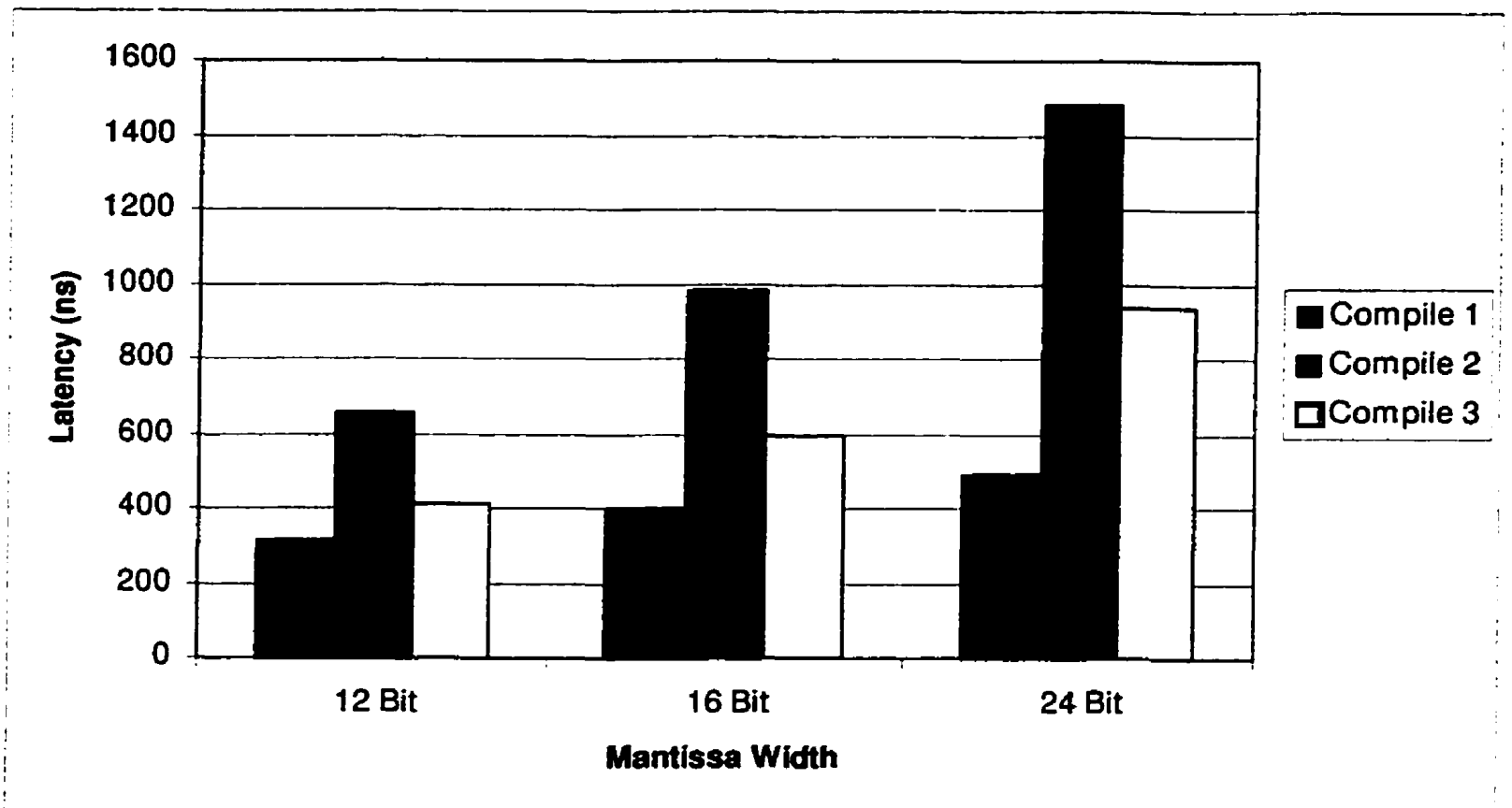

FIGURE 7.10. Latency - No Z Path, Serial / Parallel

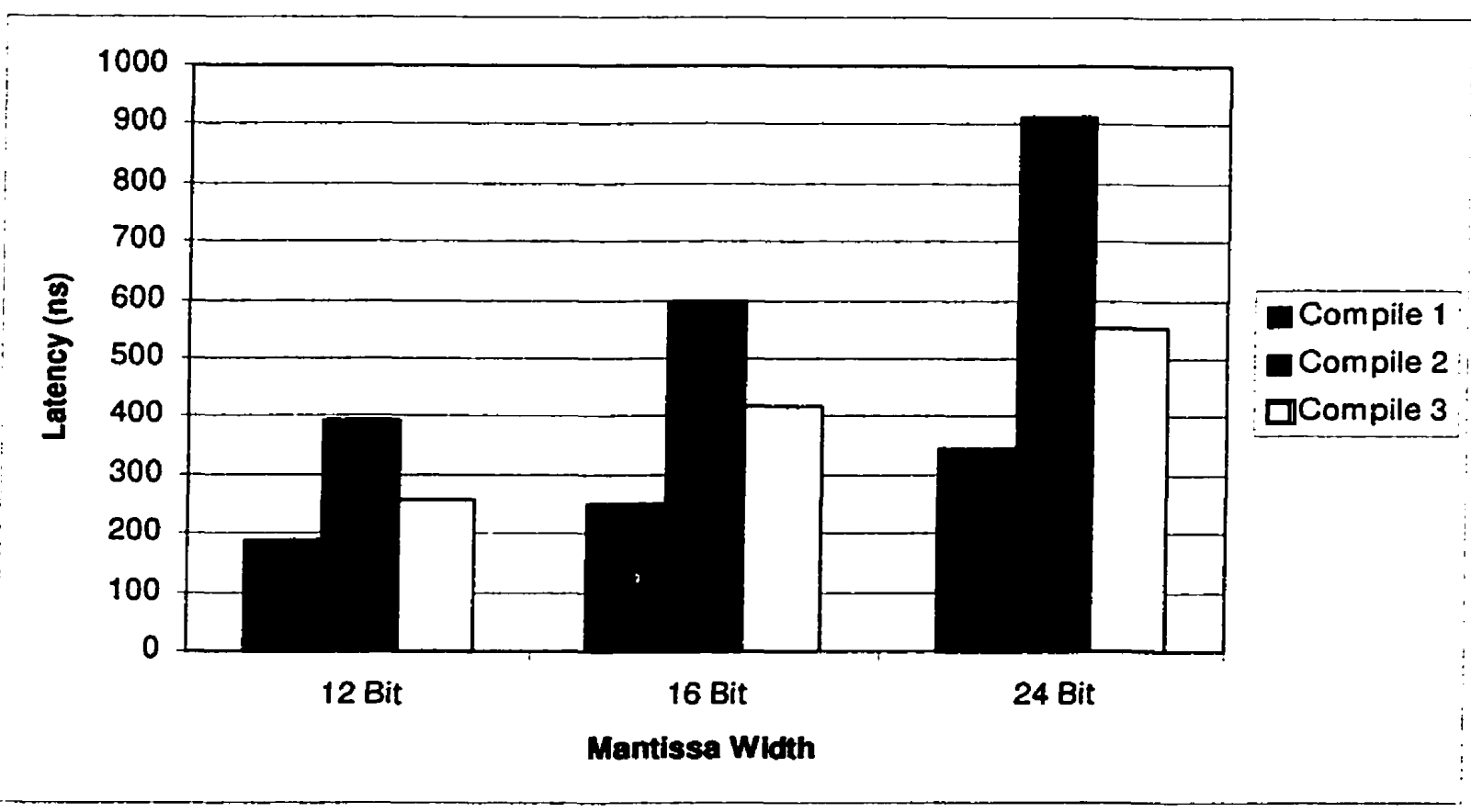

FIGURE 7.11. Latency - Z Path, Full Parallel 


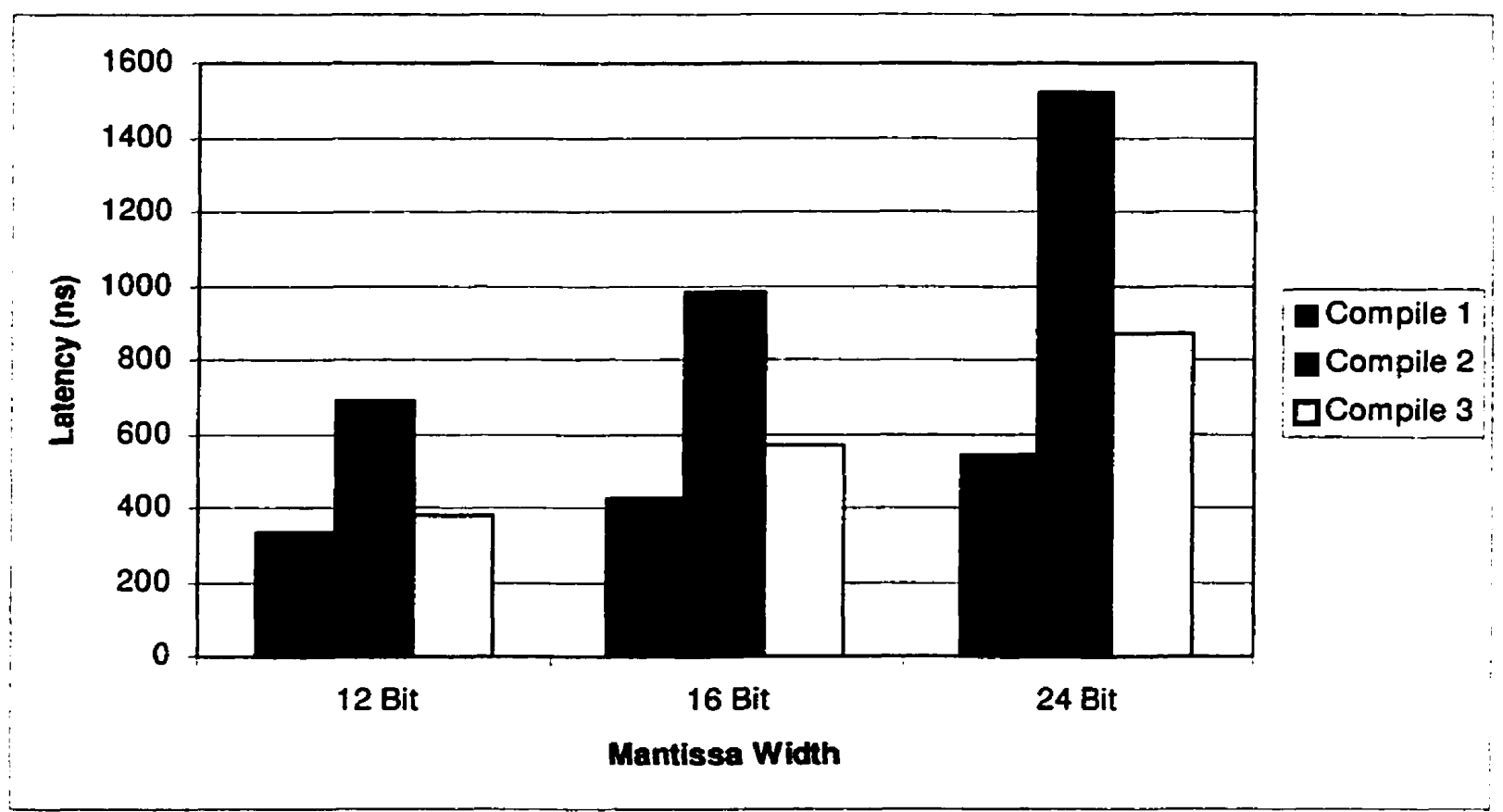

FIGURE 7.12. Latency - Z Path, Serial / Parallel

\subsection{Energy}

The energy consumption of the various versions of the CORDIC PE is shown in Figure 7.13 to Figure 7.16 and is measured in nanojoules per cycle. The energy measure is more meaningful than a power measure as it is normalized with respect to the clock speed. This normalization allows the metric to be compared between different versions of the PE with the frequency component removed. The results quoted are the average result of the three sets of input data simulated. The degree of correlation did not have a significant impact on the energy metrics. In general, the energy consumption was lower for highly correlated input data and there was greater variation between simulations for the 24 bit mantissa versions. 
The results show that the larger and faster versions of the PE use considerably more energy. This is to be expected as the fast adders used in these implementations essentially double the required components to achieve their high speed. In the larger bit width implementations there are also more stages required for the computation and so the energy required is higher. The energy consumption of the versions with a $z$ datapath is also higher, as expected, due to the increase in complexity.

The results also show that the energy requirements of the Compile 3 versions are relatively little above the requirements of the smallest versions. The significance of that is that the trade-off for a significant increase in speed is a relatively small increase in energy.

The energy requirements of the serial/parallel implementations are higher than their full parallel counterparts due to the increase in circuit complexity. The serial/parallel pipeline stages have the additional feedback registers which the full parallel versions do not. Additionally, there is the effect of the non-ideal mapping of the iterations in the serial parallel versions which result in an increased energy consumption due to the additional iterations of zero value added to match the computation to the pipeline stages. This increase in energy consumption is another trade-off for the lower area of the serial/parallel versions. In practical terms this increase in energy is not as bad as it seems since the serial/ parallel versions run at slower clock speeds. The reduction in clock speed results in a lower overall power consumption for the serial/parallel versions as compared to the full parallel versions. 


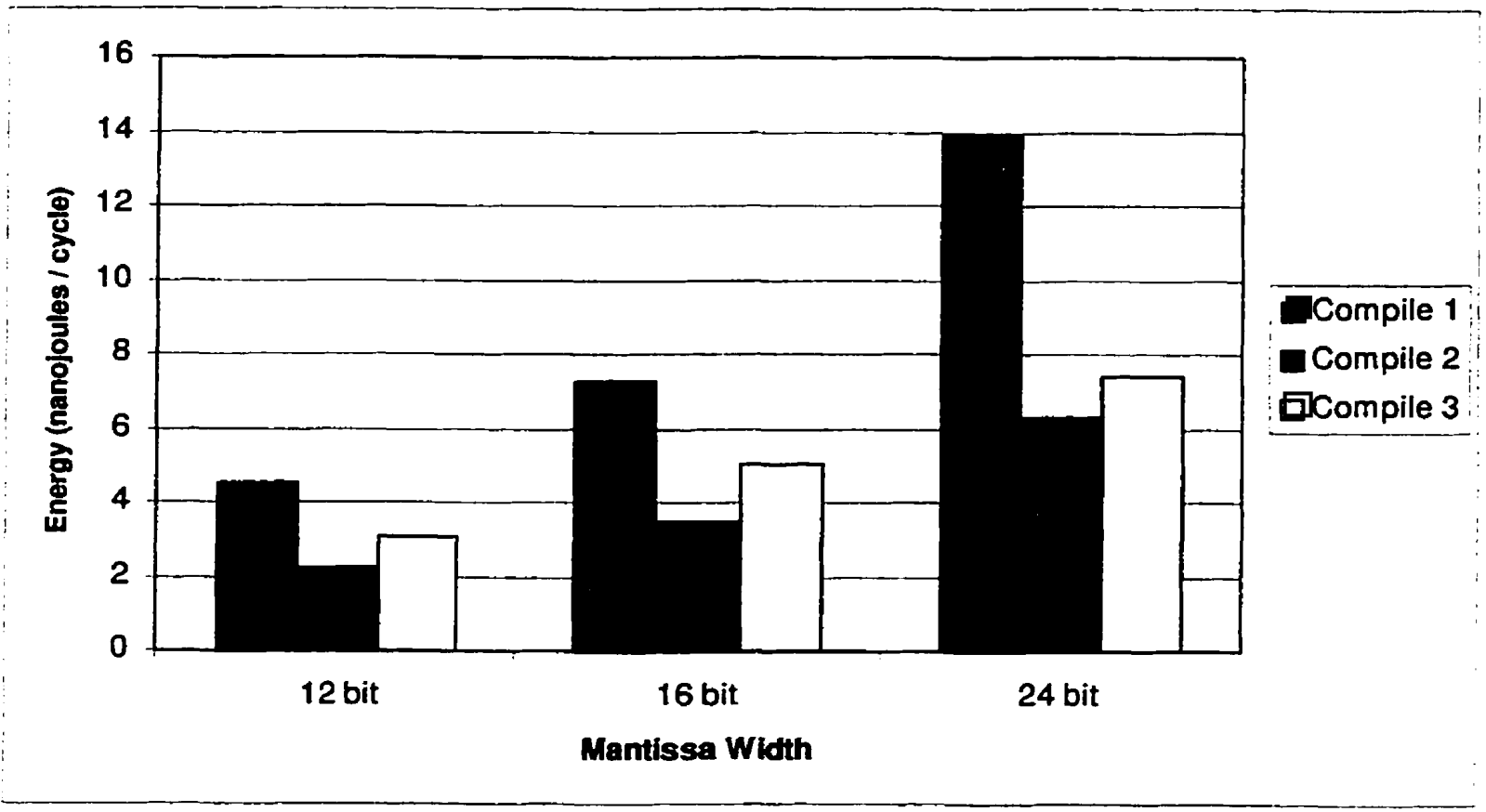

FIGURE 7.13. Energy, No Z Path, Full Parallel

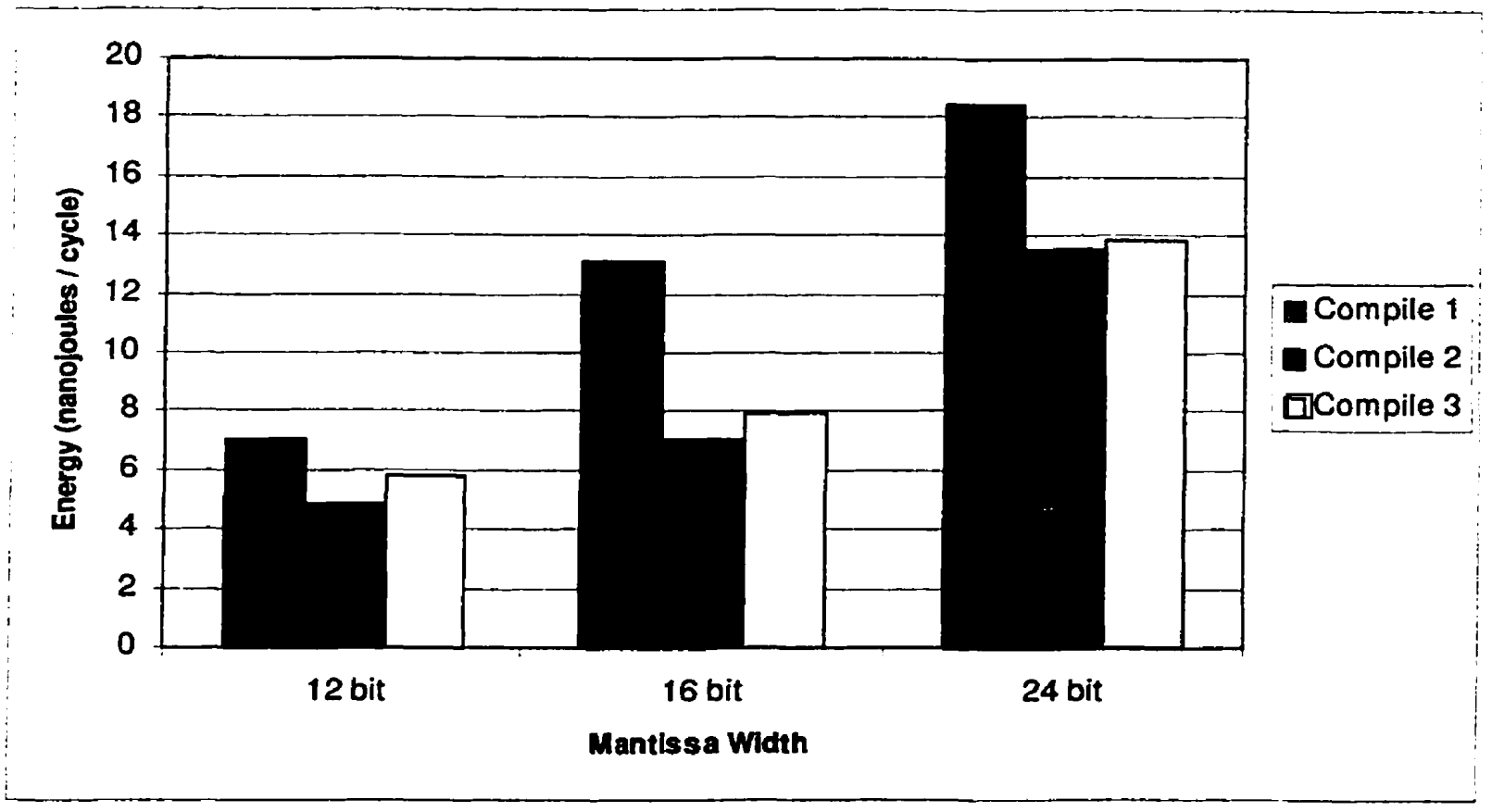

FIGURE 7.14. Energy, No Z Path, Serial / Parallel 


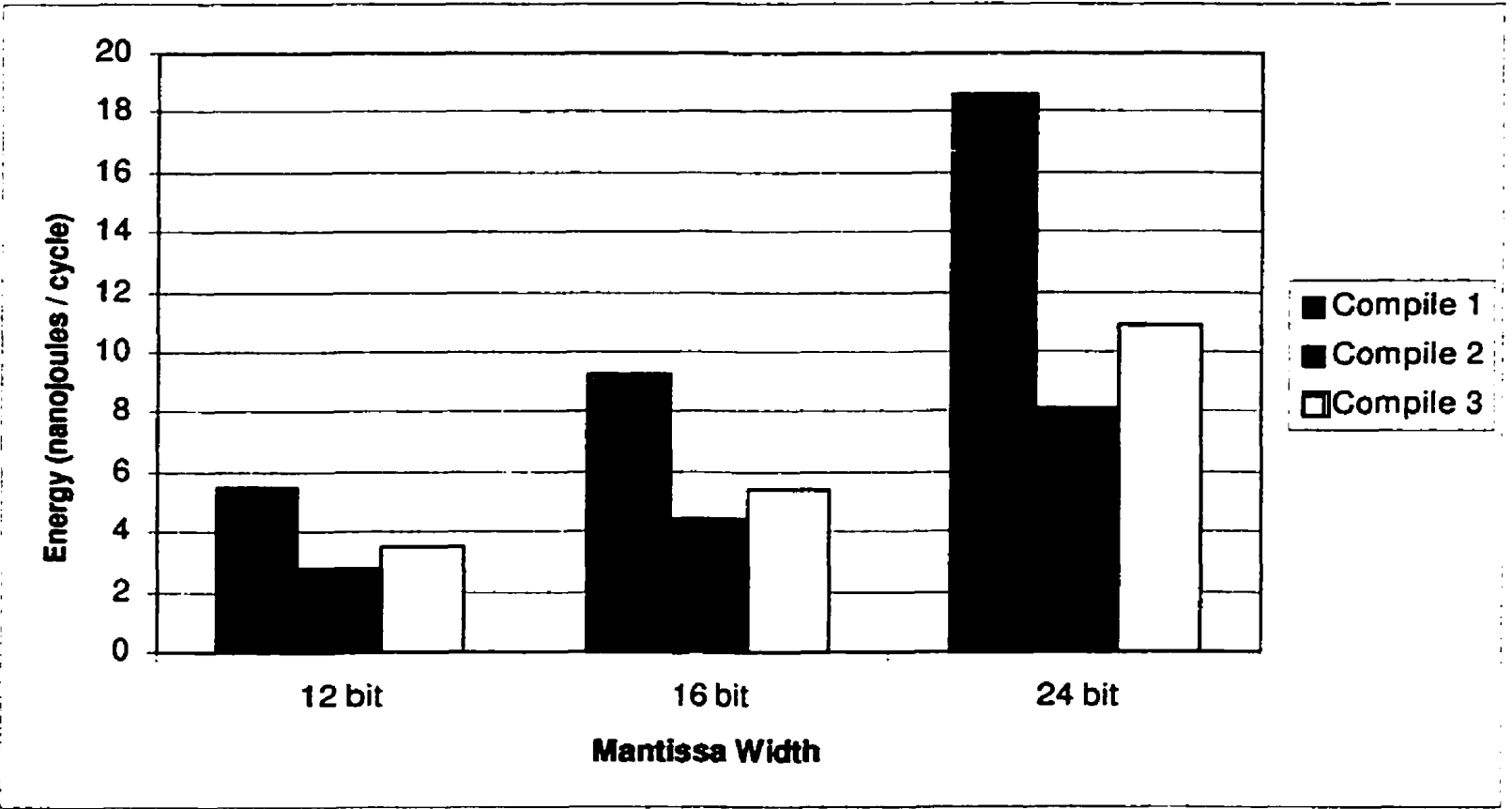

FIGURE 7.15. Energy, Z Path, Full Parallel

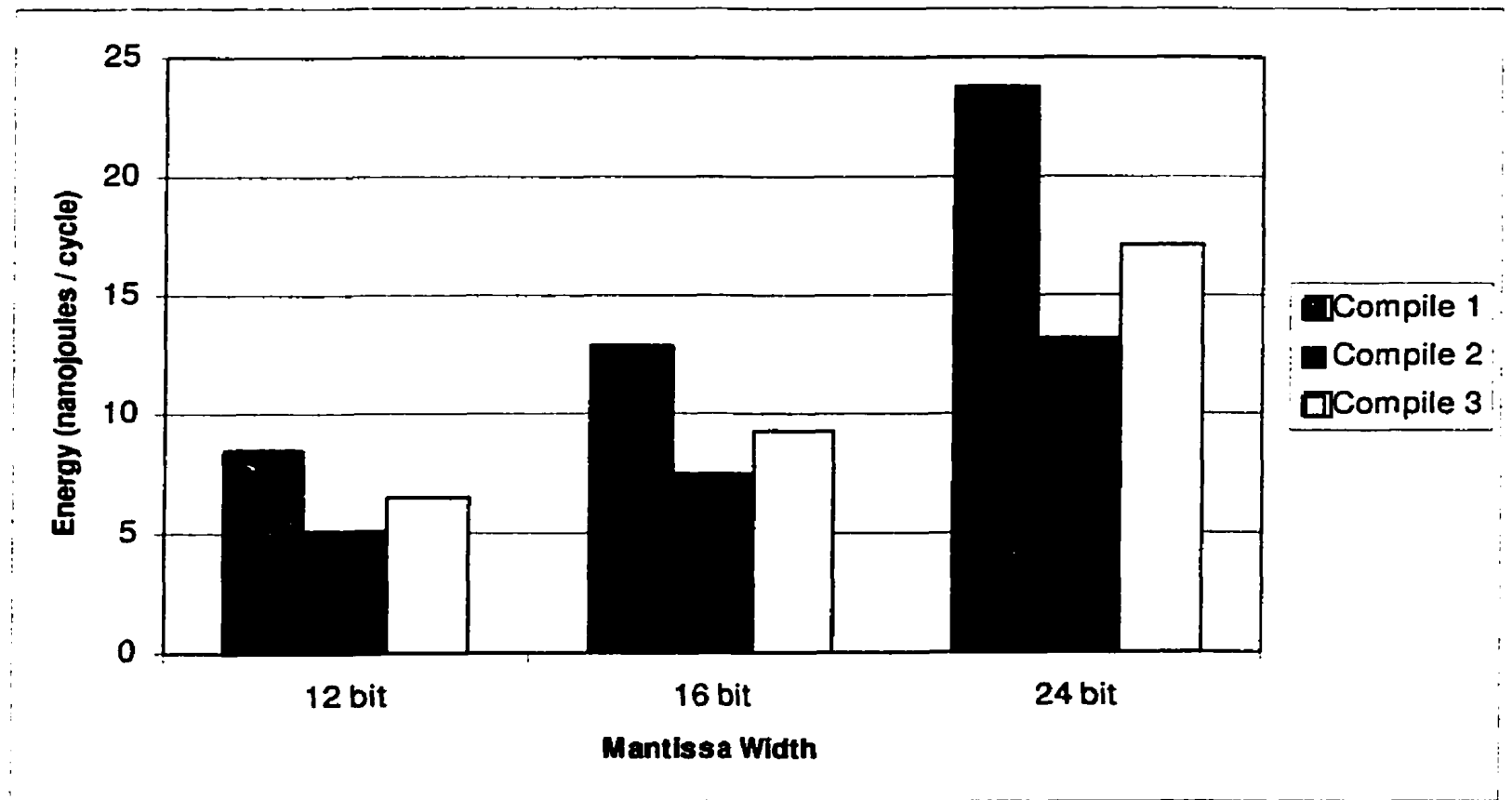

FIGURE 7.16. Energy, Z Path, Serial / Parallel 


\subsection{Energy Delay Product}

The energy delay product of the various versions of the CORDIC PE is shown in Figure 7.17 to Figure 7.20 and is measured in femto joule-seconds per cycle. The energy delay product provides a basis of comparison between implementations with respect to the benefits of trade-offs between energy and delay. The results exhibit much less variation between compilations than with the previous metrics.

The energy delay product is lower for the full parallel implementations than the serial/parallel ones by roughly a factor of ten. This indicates that the full parallel implementations are more efficient in their operation at least in terms of delay and energy. This is expected since the full parallel versions produce results more often, in other words has a higher throughput, than the serial/parallel versions despite the fact that both must perform the same computations to produce the result.

In many of the versions the lowest energy delay product was achieved with Compile 3. This indicates that there is a better balance between the energy consumed, and the speed of the PE, achieved when the design is neither the fastest possible nor the smallest possible. 


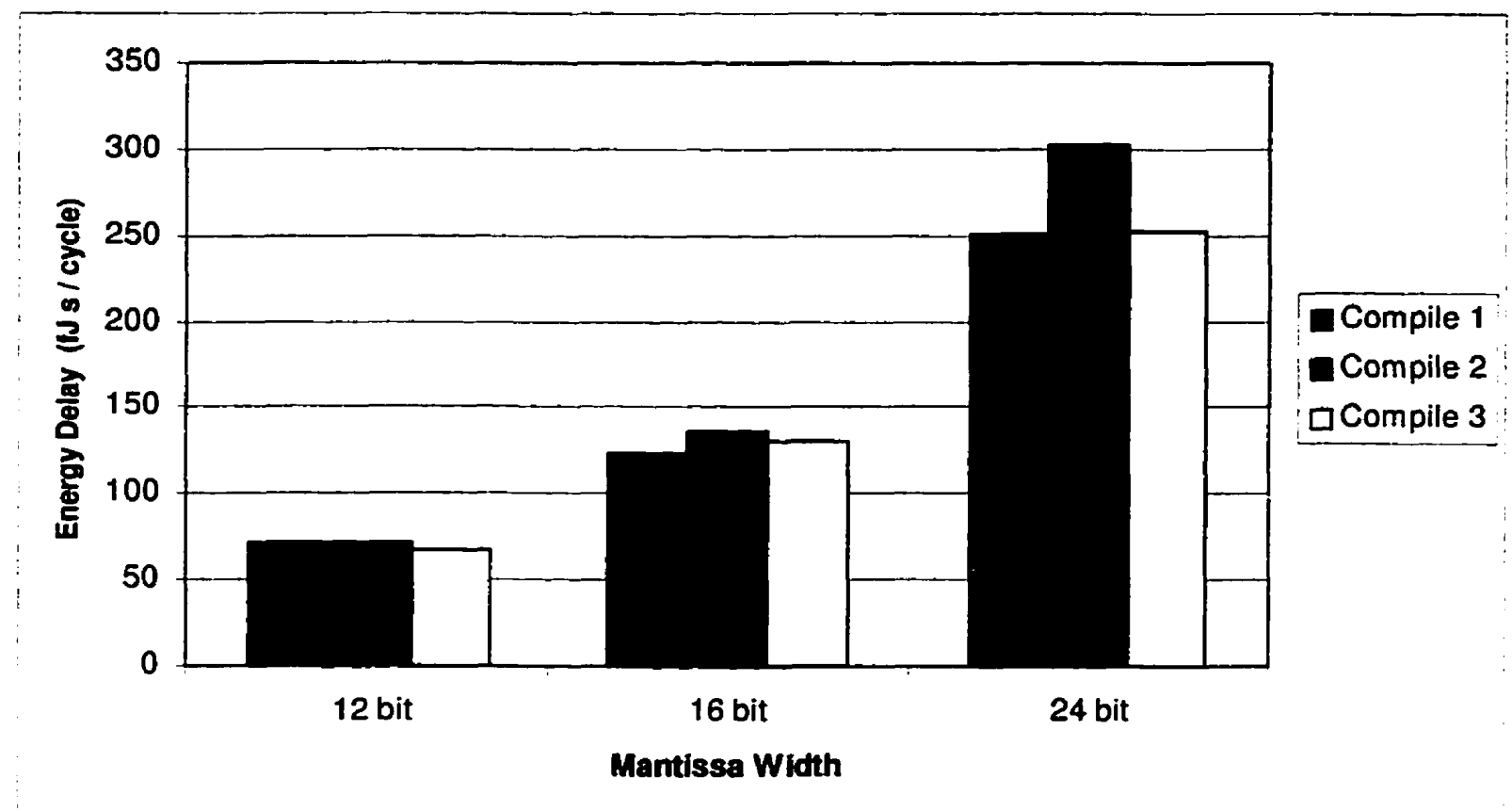

FIGURE 7.17. Energy*Delay Product, No Z Path, Full Parallel

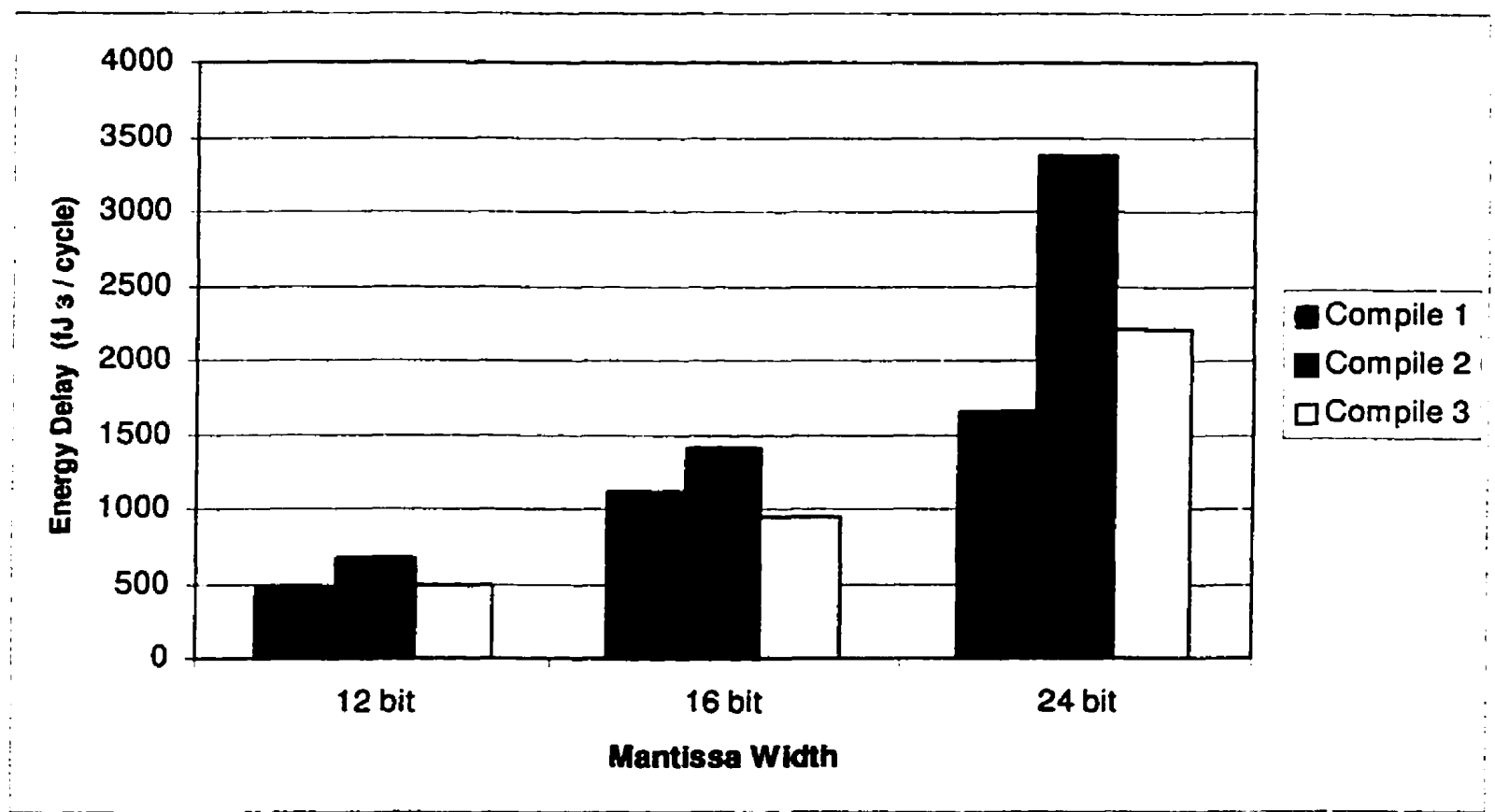

FIGURE 7.18. Energy*Delay Product, No Z Path, Serial / Parallel 


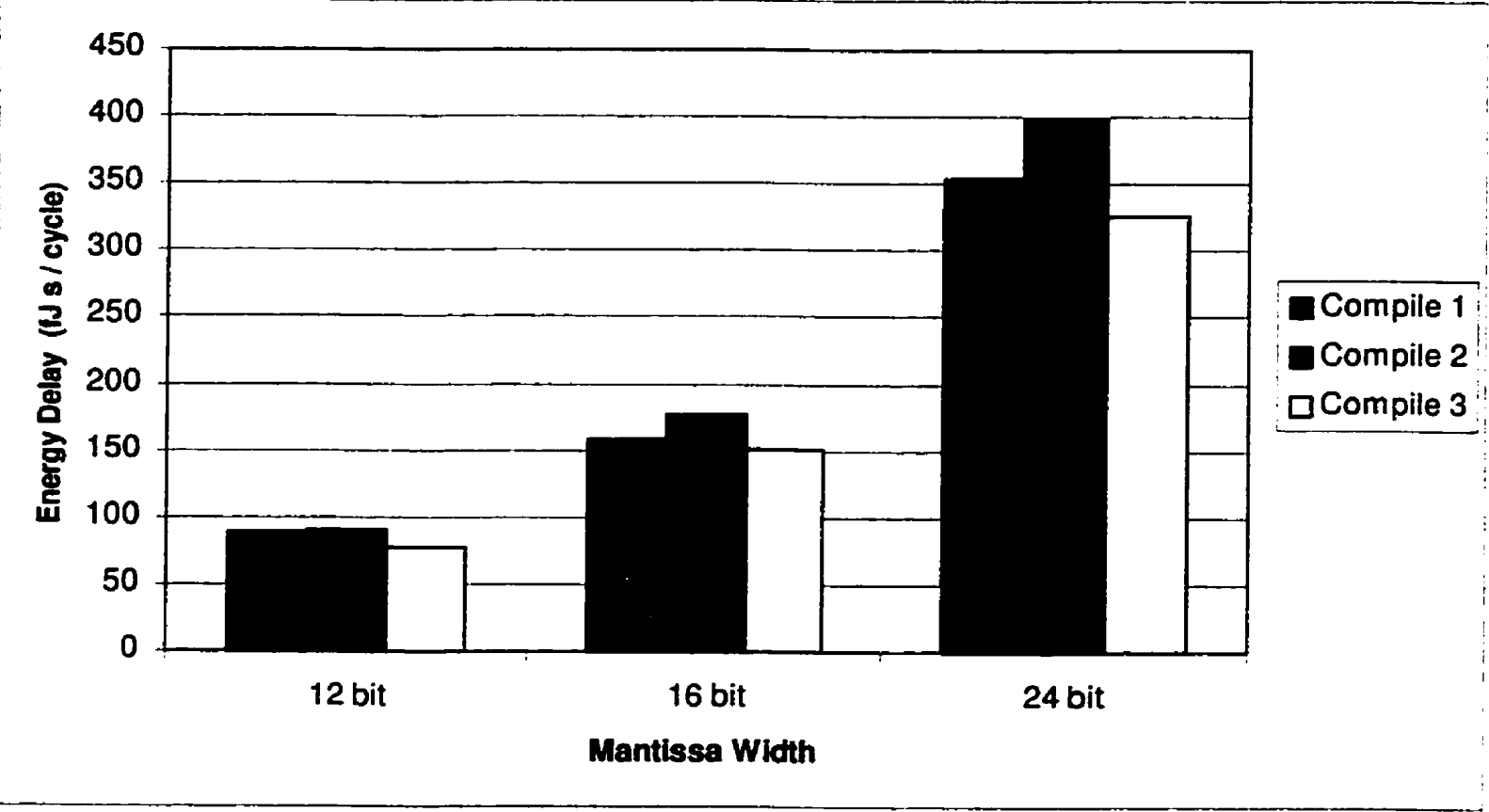

FIGURE 7.19. Energy`Delay Product, Z Path, Full Parallel

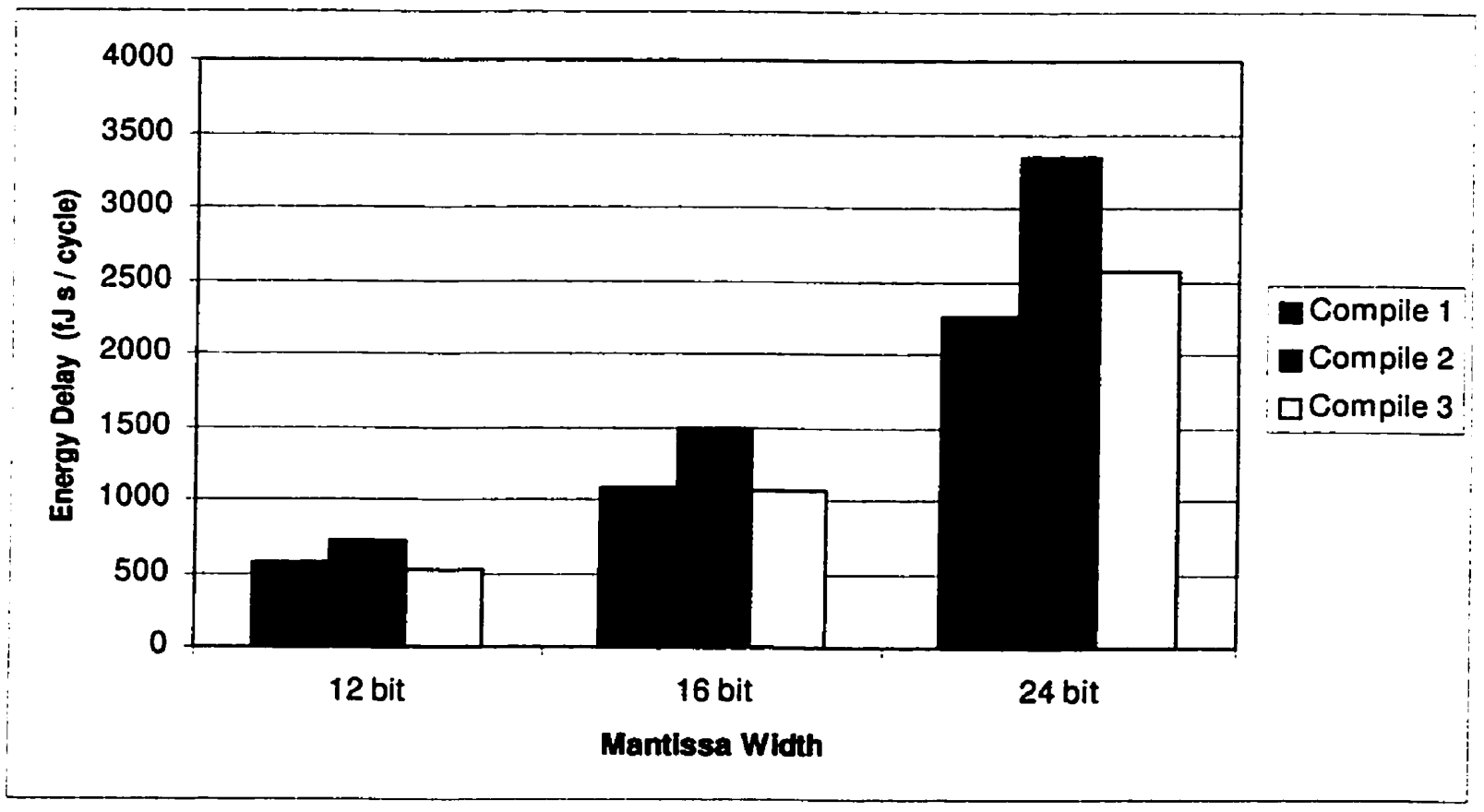

FIGURE 7.20. Energy*Delay Product, No Z Path, Serial / Parallel 


\subsection{Energy Area Product}

The energy area product of the various versions of the CORDIC PE is shown in Figure 7.21 to Figure 7.24 and is measured in nanojoules micrometers ${ }^{2}$ per cycle. The energy area product, similar to the energy delay product, provides a basis for comparison between implementations with respect to the benefits and trade-offs between energy and area.

The energy area product is lower for the serial/parallel implementations than the full parallel ones. This indicates that the serial/parallel implementations are more efficient in their operation in terms of energy and area. This is due to the fact that, although they consume more energy, they require much less area. The energy area product is higher for the versions that include the $z$ datapath but this is expected due to the increase in both area and energy consumption that the $z$ datapath requires.

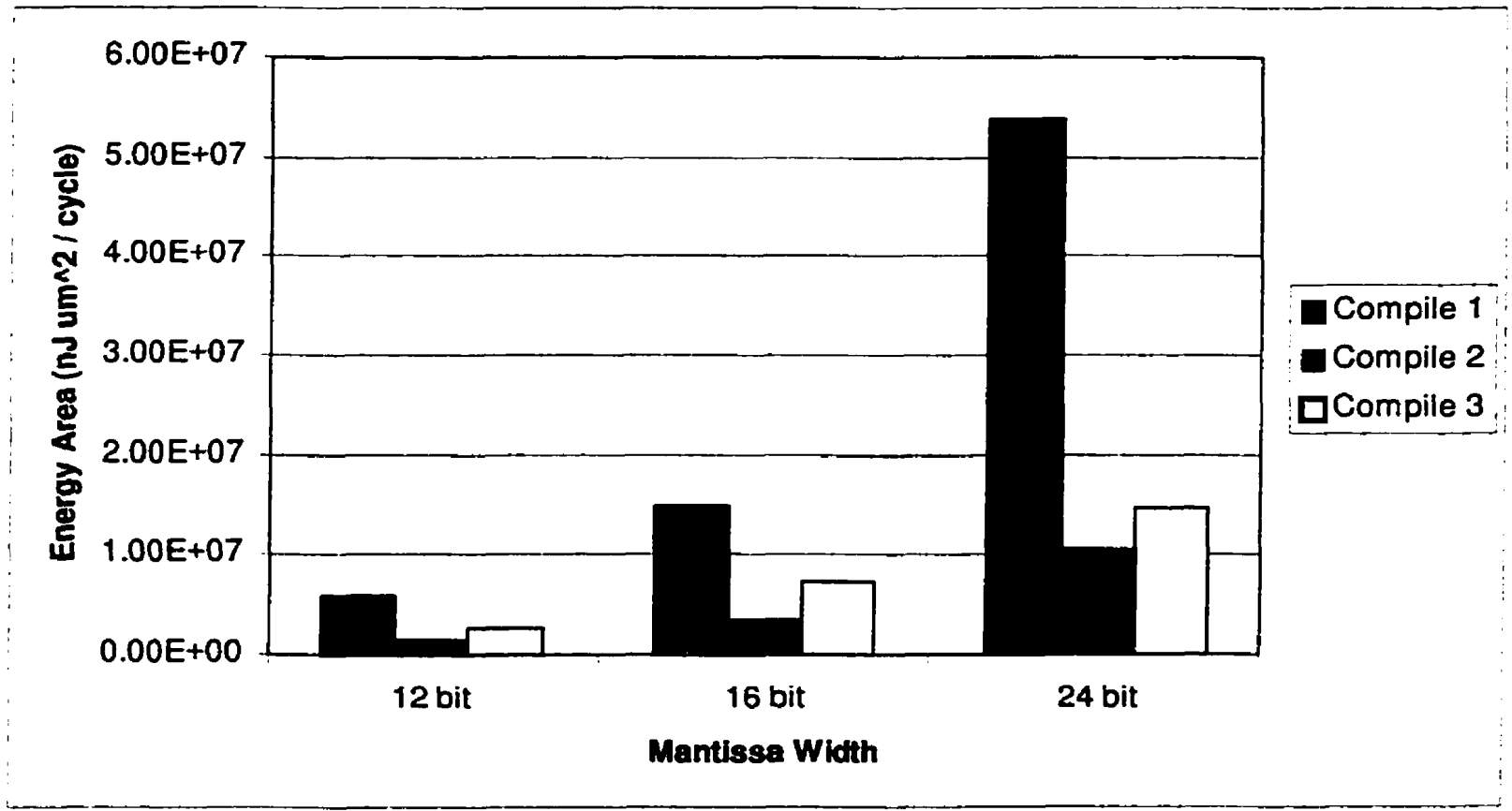

FIGURE 7.21. Energy*Area Product, No Z Path, Full Parallel 


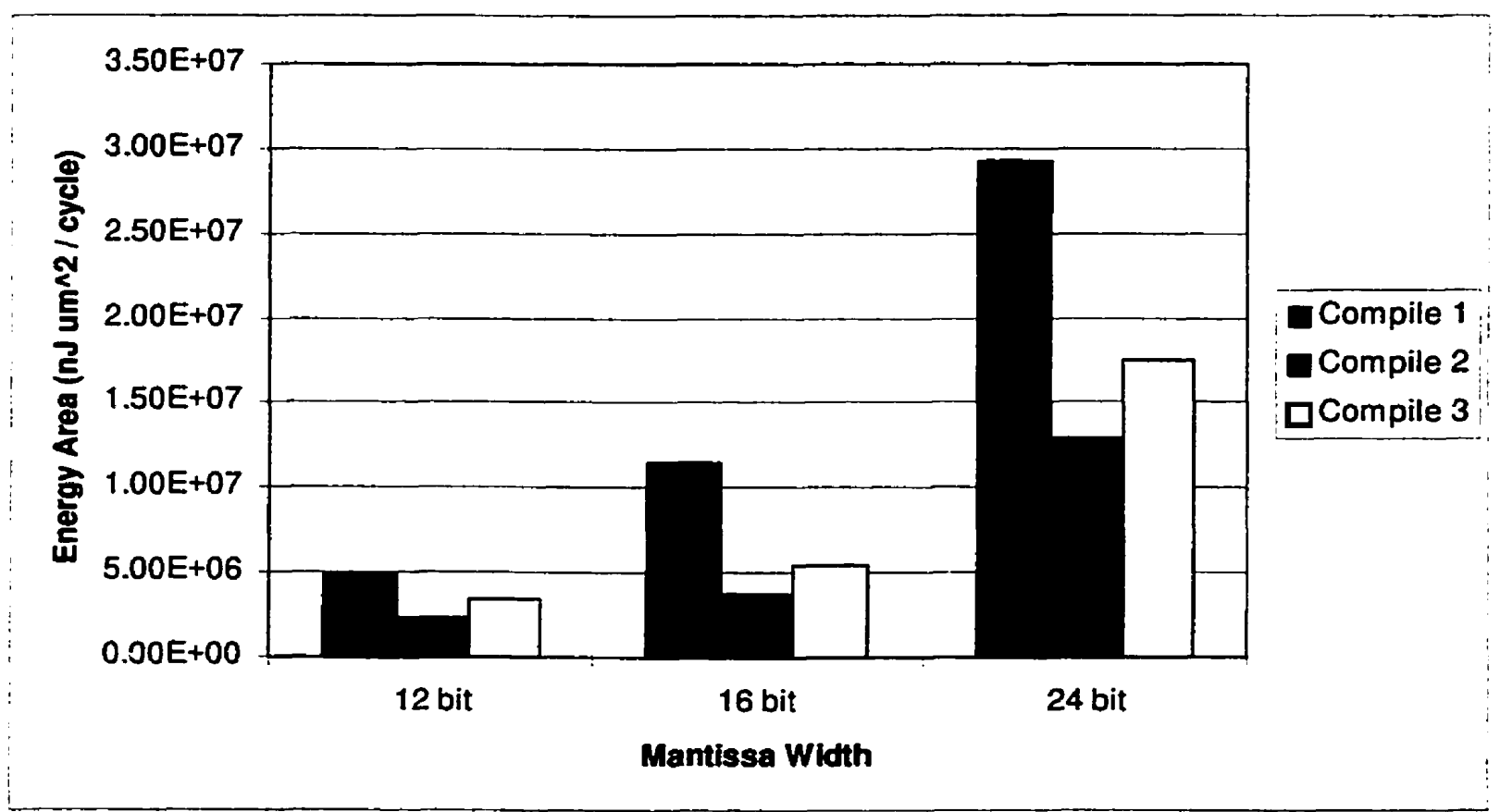

FIGURE 7.22. Energy*Area Product, No Z Path, Serial / Parallel

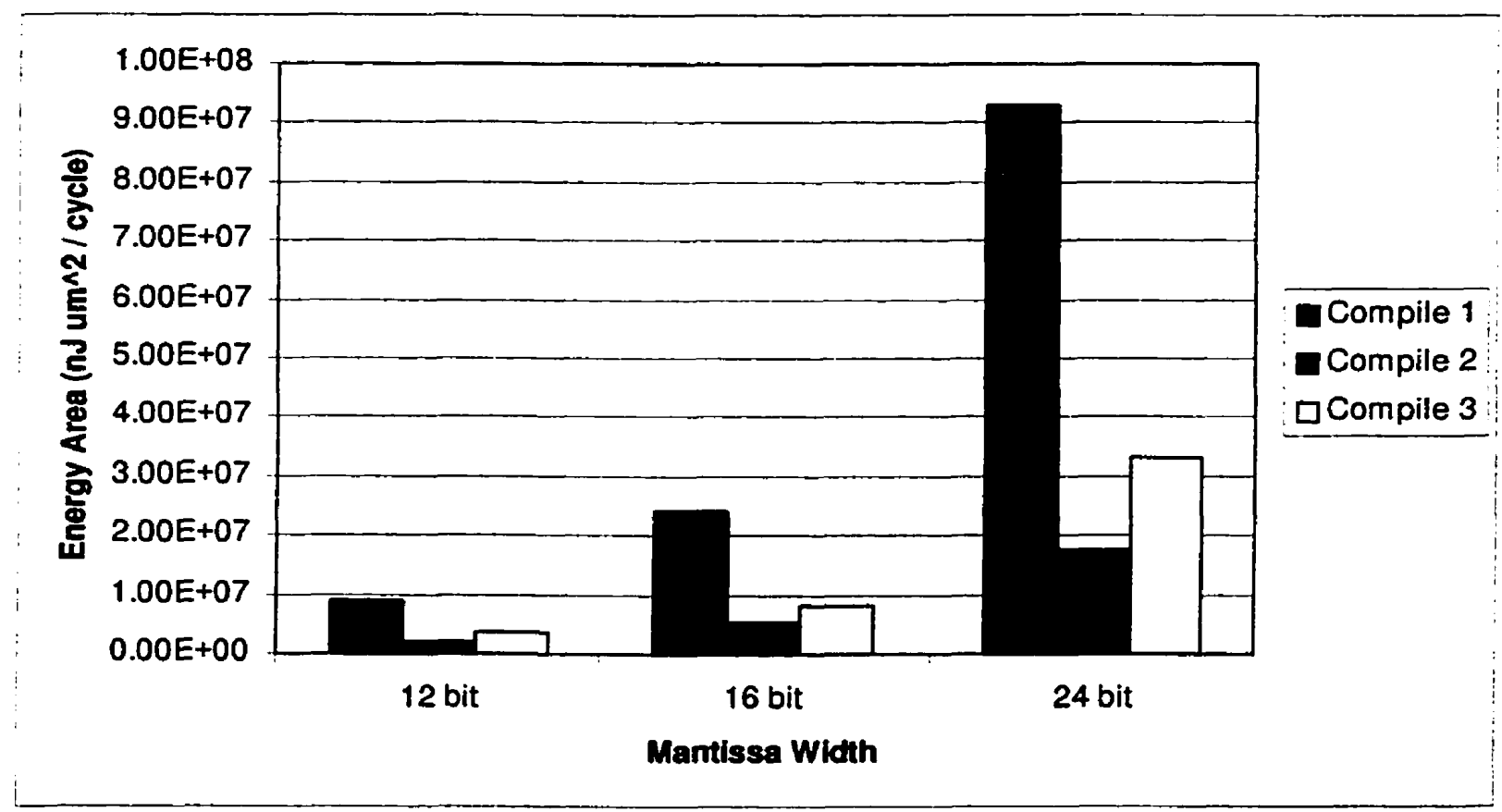

FIGURE 7.23. Energy*Area Product, Z Path, Full Parallel 


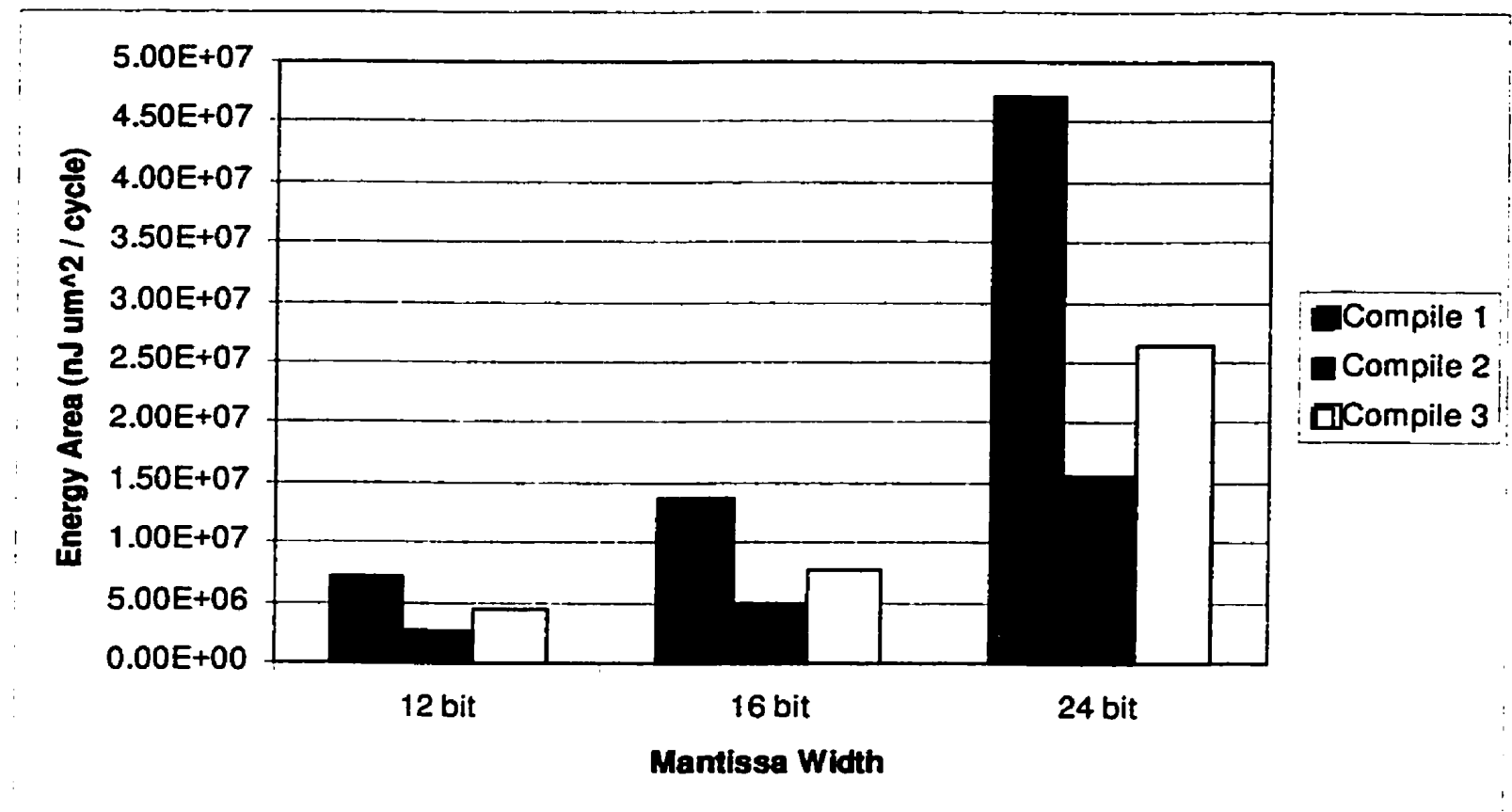

FIGURE 7.24. Energy*Area Product, Z Path, Serial / Parallel

\subsection{Pre Processor Version I Metrics}

Version I of the preprocessor alignment sub-block was characterized in order to provide an indication of the benefits of its activity scaling approach over the non-activity scaled version. This characterization was performed on the 24 bit mantissa version where the greatest impact could be experienced. As discussed with the architecture of the subblock, the benefits of the activity scaled version is data dependent on both the widths of the mantissa and exponent values as well as the correlation between the inputs. It is left to the designers to determine which version of the sub-block provides the best performance for their specific application. 
The 24 bit version of the sub-block was characterized within a fully functional PE. The mode of operation, the pipeline style and the existence of the $z$ datapath have no impact on the metrics since these factors are not relevant to the alignment function. The results of the characterization simulations is presented in Table 7.2. The data in the table is presented in both absolute quantities and normalized so that a better comparison may be made. The normalized results are obtained by comparison with the non-activity scaled version of the sub-block operating under the same conditions and with the same data set. The

\begin{tabular}{|c|c|c|c|c|c|c|c|c|}
\hline \multirow{4}{*}{$\begin{array}{c}\text { Ver- } \\
\text { sion }\end{array}$} & \multicolumn{4}{|c|}{ Measured Values } & \multicolumn{3}{c|}{ Normalized Values } \\
\cline { 2 - 9 } & $\begin{array}{c}\text { Compile } \\
\text { for } \\
\text { Speed }\end{array}$ & $\begin{array}{c}\text { Compile } \\
\text { for } \\
\text { Area }\end{array}$ & $\begin{array}{c}\text { Compile } \\
\text { for } \\
\text { Speed }\end{array}$ & $\begin{array}{c}\text { Compile } \\
\text { for } \\
\text { Area }\end{array}$ & $\begin{array}{c}\text { Compile } \\
\text { for } \\
\text { Speed }\end{array}$ & $\begin{array}{c}\text { Compile } \\
\text { for } \\
\text { Area }\end{array}$ & $\begin{array}{c}\text { Compile } \\
\text { for } \\
\text { Speed }\end{array}$ & $\begin{array}{c}\text { Compile } \\
\text { for } \\
\text { Area }\end{array}$ \\
\hline \hline I & 77402 & 45237 & 0.7713 & 0.1818 & 1.26 & 1.32 & 0.8365 & 0.8440 \\
\hline II & 61285 & 34160 & 0.9220 & 0.2154 & 1 & 1 & 1 & 1 \\
\hline
\end{tabular}

\section{TABLE 7.2. Preprocessor Version I and II Metrics}

simulation data was randomly generated and was scaled to covered the entire range of values. The power savings for Version I depend on the probability of shift fabric bypass conditions and so the complete range of exponents was required to properly exercise the preprocessor.

It is clear from the data that there is energy saved by holding the inputs to the shift fabric constant when they are not required. The amount of energy saved is reduced by the amount required for the additional control logic and the clock delay circuit. In this simulation the delay circuit was composed of buffers and inverters. Some commercial libraries feature delay elements which exhibit superior power versus delay performance. Using 
these elements in place of normal gates will produce additional power savings for Version

I. The relative energy savings are less in the version compiled for area because of the clock delay element. The smaller version produces its control signals slower than the compilation for speed version, and so the clock must be delayed by a greater amount. The extra delay requires more cells in the delay circuit, which consume more energy, and reduce the realized energy savings.

\subsection{Post Processor Version I Metrics}

Version I of the postprocessor was characterized in order to provide an indication of the costs of performing normalization of the outputs. This characterization was also performed on the 24 bit mantissa version where the greatest impact could be experienced. As discussed with the architecture of the block, the costs of the normalization include increased design complexity and area. Normalization does not provide any increase in accuracy and may in fact lead to the false assumption that it does. Errors are generated in the iteration pipeline and are, therefore, relative to the working exponent of the data and not that of the outputs. It is not advantageous in terms of performance to perform normalization and it should not be used for results being sent to a subsequent PE. Normalization may be required by design components interfacing with the $\mathrm{PE}$ and that is why the functionality has been provided.

The 24 bit version of the block was characterized within a fully functional PE operating in the vectoring mode. The pipeline style and the existence of the $z$ datapath have no impact on the metrics since these factors are not relevant to the normalization 
function. While the mode of operation has no impact on the area of the postprocessor, it does have an impact on the energy consumption. In the vectoring mode of operation, the $y$ mantissa is driven to zero. This results in a low energy shif, if one is required at all, for its normalization process since at most one bit of the mantissa is non-zero. In the rotation mode, however, the energy consumption of the postprocessor may be higher since the $y$ mantissa is no longer driven to zero and a shift of many non-zero bits may be required. The results of the characterization simulations are presented in Table 7.3. The data in the table is presented in both absolute quantities and normalized so that a better comparison may be made. The normalized results are obtained by comparison with the non-normalizing version of the block operating under the same conditions and with the same data set.

\begin{tabular}{|c|c|c|c|c|c|c|c|c|}
\hline \multirow{4}{*}{$\begin{array}{c}\text { Ver- } \\
\text { sion }\end{array}$} & \multicolumn{4}{|c|}{ Measured Values } & \multicolumn{3}{c|}{ Normalized Values } \\
\cline { 2 - 9 } & $\begin{array}{c}\text { Area }\left(\mu \mathbf{m}^{2}\right) \\
\begin{array}{c}\text { Compile } \\
\text { for } \\
\text { Speed }\end{array}\end{array}$ & $\begin{array}{c}\text { Compile } \\
\text { for } \\
\text { Area }\end{array}$ & $\begin{array}{c}\text { Compile } \\
\text { for } \\
\text { Speed }\end{array}$ & $\begin{array}{c}\text { Compile } \\
\text { for } \\
\text { Area }\end{array}$ & $\begin{array}{c}\text { Compile } \\
\text { for } \\
\text { Speed }\end{array}$ & $\begin{array}{c}\text { Compile } \\
\text { for } \\
\text { Area }\end{array}$ & $\begin{array}{c}\text { Compile } \\
\text { for } \\
\text { Speed }\end{array}$ & $\begin{array}{c}\text { Compile } \\
\text { for } \\
\text { Area }\end{array}$ \\
\hline \hline I & 182787 & 87045 & 0.2506 & 0.0915 & 2.71 & 2.57 & 1.536 & 1.378 \\
\hline II & 67462 & 33775 & 0.1631 & 0.0664 & 1 & 1 & 1 & 1 \\
\hline
\end{tabular}

TABLE 7.3. Postprocessor Version I and II Metrics

It is clear from the data that the normalization of the output mantissas does not come cheap in relative terms. As a comparison, the total energy consumption of a the PE with the non-normalizing postprocessor is 18.5 and 13.6 nanojoules/cycle for the compiles for speed and area respectively. The absolute increase in energy is not large when compared to the total energy bill but, it is still an increase, and need not be incurred for most applications. 


\section{CHAPTER 8}

\section{CONCLUSION AND RECOMMENDATIONS}

\subsection{Conclusion}

This thesis presented the development of a CORDIC processing element module suitable for use in embedded systems and SoC applications. The CORDIC algorithm was investigated and recent proposed modifications were evaluated. A version of the algorithm suitable for general purpose DSP applications was selected and work was conducted to apply it for the design requirements. Architectures were developed for the different implementations required and then encoded as part of a behavioural synthesis tool that was developed as part of this work. The tool allows a designer to produce a CORDIC processing element which is tailored for use in a particular application and which meets the design requirements. The tool can produce a total of 576 different implementations of the processing element, all of which are complete Verilog ${ }^{\circledR} \mathrm{HDL}$ models ready for synthesis.

The output data produced by the CORDIC PE has been shown to be accurate to the full input data width for, on average, $98.8 \%$ of the input samples. The error in the remaining $1.2 \%$ has been shown to be restricted to the least significant bit. The range of CORDIC PE versions occupied an area of between $635,000 \mu^{2}$ and $5,000,000 \mu^{2}$, had maximum 
throughputs of 42.4 to 64.4 million samples per second and consumed energy at 2.27 to 23.8 nanoJoules per cycle using TSMC's $0.35 \mu$ CMOS technology.

\subsection{Recommendations for Future Work}

The work in this thesis can be expanded on in a number of areas.

Many modern DSP algorithms can make use of the hyperbolic coordinate system. This is not currently supported by the CORDIC Behavioural Synthesis Tool, but that functionality could be implemented within the existing framework. This would require an examination of the hyperbolic CORDIC algorithm, an investigation of an optimal set of iteration parameters and coding of the computational pipeline stages into the tool. The architectures that were developed for the circular coordinate system as part of this work are also valid for the hyperbolic system and so this work does not need to be repeated. Expansion of the tool for the linear coordinate system is not required as multiply and accumulate (MAC) designs exhibit superior performance metrics [22].

Additional work could be completed to better determine the parameter sets for the chosen algorithm. To reduce the errors produced by the algorithm even further, a more exhaustive search method is required. The method used in this work produced large amounts of data which had to be evaluated. It may be possible to develop a directed search pattern which would make the finding and selection of the parameter set better and easier.

Design for testability is becoming one of the main requirements when designing integrated circuits. Unfortunately due to the limited time available for this project, the test- 
ability aspect was not considered. The author strongly recommends that the design should be revisited to incorporate testability features such as BIST and scan path.

Physical testing is required of any design before it can truly be said to work. The CORDIC PE designs produced by the behavioural synthesis tool are synthesizable and produce correct results under simulation. A final step would be to perform the remainder of the design flow through layout, placement and routing to the fabrication stage. The physical design could then be tested to verify its functionality.

Finally, the CORDIC PE could be implemented in a DSP or similar algorithm. A suitable application could be found, designed, fabricated, and tested so that the performance of the CORDIC implementation could be compared against other algorithm implementations. This would provide a concrete example of the benefits of the CORDIC algorithm and the Behavioural Synthesis Tool which was developed. 


\section{REFERENCES}

1. Jean-Michel Muller, "Elementary Functions" Birkhauser, 1997, Chapter 6.

2. H. Chang, L. Cooke, M. Hunt, G. Martin, A. McNelly, L. Todd, "Surviving the SOC Revolution: A Guide to Platform-Based Design," Kluwer Academic Publishers, Massachusetts, U.S.A, 1999, p. 4.

3. G. Nudd, G.J. Vaudin, "A Cellular VLSI Processing Architecture Using CORDIC Elements for Imaging Processing," Colloquium on VLSI for Image Processing, London England, 1987, pp. 3.1-3.6.

4. Jack E Volder, "The CORDIC Trigonometric Computing Technique," IRE Transactions on Electronic Computers, Vol. EC-8, No. 3, Sept. 1959, pp. 330-4.

5. J.S. Walther, "A Unified Algorithm for Elementary Functions," Proceeding, Spring Joint Computer Conference, 1971, Vol. 38, pp. 379-385.

6. S. Wang, V Piuri, E Swartzlander Jr, "Hybrid CORDIC Aigorithms," IEEE Transactions on Computers, Vol. 46, No. 11, November 1997, pp 1202-1207.

7. N. Takagi, T. Asada, S Yajima, "Redundant CORDIC Methods with a Constant Scale Factor for Sine and Cosine Computation," IEEE Transactions on Computers, Vol. 40, No. 9, September 1991, pp 989-995.

8. N. Takagi, T. Asada, S Yajima, "A Hardware Algorithm for Computing Sine and Cosine using Redundant Binary Representation," Transactions IECE Japan, Vol. J69-D, No. 6, pp. 841-847, June 1986 (in Japanese). English translation is available in Systems and Computers in Japan, Vol. 18, No. 8, pp. 1-9, August 1987.

9. S. Wang, E.E. Swartzlander, "Merged CORDIC Algorithm," Proceedings of the 1995 IEEE International Symposium on Circuits and Systems, April 1995, pp 1988-1991.

10. Julio Villalba, Thomas Lang, "Low Latency Word Serial CORDIC," Proceedings IEEE Internationai Conference on Application Specific Systems, Architectures and Processors, July 1997, pp. 124-131.

11. AJean Duprat, Jean-Michel Muller, "The CORDIC Algorithm: New Results for Fast VLSI Implementation," IEEE Transactions on Computers, Vol. 42, No. 2, February 1993, pp. 168-178.

12. Dhananjay S. Phatak, "Double Step Branching CORDIC: A New Algorithm for Fast Sine and Cosine Generation," IEEE Transactions on Computers, Vol. 47, No. 5, May 1998, pp. 587-602.

13. Dhananjay S. Phatak, "Comments on Duprat and Muller's Branching CORDIC Paper," IEEE Transactions on Computers, Vol. 47, No. 9, September 1998, pp. 1037-1040.

14. Herbert Dawid, Heinrich Meyr, "High Speed Bit-Level Pipelined Architectures for Redundant CORDIC Implementation," IEEE International Conference on Application Specific Array Processors 1992, VLSI Architectures II, pp. 358-372.

15. D. Timmermann, H. Hahn, B.J. Hosticka, "Low Latency Time CORDIC Algorithms," IEEE Transactions on Computers, Vol. 41, No. 8, August 1992, pp 1010-1015.

16. Ed F. Deprettere, P. Dewilde, R. Udo, "Pipelined CORDIC Architectures for Fast VLSI Filtering and Array Processing," IEEE International Conference on Acoustics, Speech, and Signal Processing, San Diego Califomia, March 19-21, 1984, pp. 41.A.6.1-41.A.6.4. 
17. A.A.J. de Lange, A.J. van der Hoeven, E.F. Deprettere, J. Bu, “An Optimal Floating Point Pipeline CMOS CORDIC Processor," 1988 IEEE International Symposium on Circuits and Systems, Helsinki Finland, pp 2043-2047.

18. A.A.J. de Lange, E.F. Deprettere, A. van der Veen, J. Bu, "Real Time Applications of the Floating Point Pipeline CORDIC Processor in Massive-Parallel Pipelined DSP Algorithms," IEEE International Conference on Acoustics, Speech and Signal Processing, New Mexico, U.S.A., 1990, pp. 1013-1016.

19. G. Schmidt, D Timmermann, J.F. Bohme, H. Hahn, B.J. Hosticka, and G. Zimmer, "Parameter Optimization of the CORDIC-Algorithm and implementation in a CMOS-Chip," Signal Processing III : Theories and Applications: Proceedings of the EUSIPCO-86 Third European Signal Processing Conference, Netherlands, pp. 1219-1222.

20. Julio Villalbe, Thomas Lang, E.L. Zapata, "Parallel Compensation of Scale Factor for the CORDIC Algorithm," Journal of VLSI Processing 19, 1998, pp. 227-241.

21. K. Hwang, “Computer Arithmetic Principles, Architecture, and Design," John Wiley \& Sons, Inc., New York, 1979.

22. Yu Hen Hu, "CORDIC-Based VLSI Architectures for Digital Signal Processing," IEEE Signal Processing Magazine, July 1992, pp. 16-35.

23. G.L. Haviland, A.A. Tuszynski, "A CORDIC Arithmetic Processor Chip," IEEE Transactions on computers, Vol. C-29, No. 2, pp 68-79, February 1980.

24. Yu Hen Hu, "The Quantization Effects of the CORDIC Algorithm," IEEE Transactions on Signal Processing, Vol. 40, No. 4, April 1992, pp 834-844.

25. H.M. Ahmed, "Signal Processing Algorithms and Architectures," Ph.D Dissertation, Department of Electrical Engineering, Stanford University, Stanford, CA, June 1982.

26. E. Antelo, J.D. Bruguera, T. Lang, E.L. Zapata, "Error Analysis and Reduction for Angle Calculation Using the CORDIC Algorithm," IEEE Transactions on Computers, Vol. 46, No. 11, November 1997, pp 1264-1271.

27. K Kota, J.R. Cavallaro, "Numerical Accuracy and Hardware Tradeoffs for CORDIC Arithmetic for Special Purpose Processors," IEEE Transactions on Computers, Vol. 42, No. 7, July 1993, pp. 769-779.

28. X. Hu, R.G. Harber, S.C. Bass, "Expanding the Range of Convergence of the CORDIC Algorithm," IEEE Transactions on Computers, Vol. 40, No. 1, January 1991, pp 13-21.

29. J.R. Cavallaro, F.T. Luk, "Floating-Point CORDIC for Matrix Computations," 1988 IEEE International Conference on Computer Design, VLSI in Computers and Processors, Rye Brook, New York, October 35 1988, pp. 40-42.

30. D.E. Metafas, C.E. Goutis, “A Floating Point Pipeline CORDIC Processor with Extended Operation Set," 1991 IEEE International Symposium on Circuits and Systems, Singapore, 11-14 June 1991, pp 3066-3069.

31. D. Timmermann, H. Hahn, B.J. Hosticka, "A CORDIC-Based Floating-Point Arithmetic Unit," IEEE 1992 Custom Integrated Circuits Conference, pp. 30.3.1-30.3.4.

32. S.C. Bass, G.M. Butler, R.L. Williams, F. Barlos, D.R. Miller, “A Bit-Serial, Floating Point CORDIC Processor in VLSI," 1991 International Conference on Acoustics, Speech and Signal Processing, Toronto Ontario, May 14-17, 1991, pp. 1165-1168.

33. A.A.J. de Lange, E.F. Deprettere, "Design and Implementation of a Floating Point Quasi-Systolic General Purpose CORDIC Rotator for High-Rate Parallel Data and Signal Processing," Proceedings 10th IEEE Symposium on Computer Arithmetic, Grenoble France, June 26-28 1991, pp. 272-281.

34. P. Chown, D.W. Walton, G.R. Nudd, "VLSI Design of a Pipelined CORDIC Processor," University of Warwick, Department of Computer Science Research, United Kingdom, Report \# 164, October 1990. 
35. G. J. Hekstra, E.F.A. Deprettere, "Floating Point CORDIC, extended version," Delft University of Technology, Department of Electrical Engineering, The Netheriands, Technical Report ET/NT 93.15 dated 8 March 1993.

36. T.C. Davies, "A Floating-Point Systolic Array Processing Element Using Serial Communication," Masters Thesis, Department of Electrical and Computer Engineering, Royal Military College of Canada, 1990, pp. 2-19-2-21.

37. Anatha P. Chandrakasan, Robert W. Brodersen, "Low Power Digital CMOS Design," Kluwer Academic Publishers, Massachusetts, U.S.A, 1995, pp. 235-241.

38. A. Lacroix, F Hartwig, "Distribution Densities of the Mantissa and Exponent of Floating Point Numbers," IEEE International Symposium on Circuits and Systems, San Diego, CA, May 10-13, 1992, pp. 1792-1795.

39. R.V.K. Pillai, "Report on the Design of a Low Power Barrel Switch," Internal Report, Concordia University, Montreal, Canada, 1998.

40. Hiroaki Suzuki, Hiroyuki Morinaka, Kiroshi Makino, Yasunobu Nakase, Koichiro Mahiko and Tadashi Sumi, "Leading-Zero Anticipatory Logic for High-Speed Floating Point Addition," IEEE Journal of Solid State Circuits, Vol.31, No. 8, August 1996, pp1157-1164.

41. Synopsys Inc, "Behavioral Compiler User Guide: Overview and Mehtodology," v1998.08, Chapter 1.

42. Joe Costello, "Technical Report: CORDIC Behavioural Synthesis Tool," Internal Report, Royal Military College of Canada, Kingston, Ontario, 2000.

43. Canadian Micro Electronics Corporation, "0.35-micron CMOS (CMOSP35) Design Kit for Synopsys and Cadence V4.2," Kingston Ontario, Canada, http://www.cmc.ca/Announcements/MTB/ se0287mtb_cmosp35.4.2.html 


\section{BIBLIOGRAPHY}

1. Alvin M. Despain, "Fourier Transform Computers Using CORDIC Iterations," IEEE Transactions on Computers, Vol. C-23, No. 10, October 1974, pp 993-1001.

2. H.M. Ahmed, P.H. Ang, M. Morf, “A VLSI Speech Analysis Chip Set Utilizing Co-ordinate Rotation Arithmetic," IEEE, pp 737-741, 1981.

3. H.M. Ahmed, M. Morf, D.T. Lee, P.H. Ang, "A VLSI Speech Analysis Chip Set Based on Square-Root Normalized Ladder Forms," Proceedings 1981 International Conference on Acoustics, Speech and Signal Processing, Atlanta GA, March 30, 1981.

4. E. Antelo, J.D. Bruguera, "Error Analysis and Reduction for Angle Calculation Using the CORDIC Algorithm," IEEE Transactions on Computers, Vol. 46, No. 11, November 1997, p 1264-1271.

5. Jie Chen, K.J. Ray Liu, "A Fully Pipelined Parallel CORDIC Architecture for Half-Pel Motion Estimation," IEEE International Conference on Image Processing, Santa Barbara, California, September 26-29, 1997, pp. 574-577.

6. Jie Chen, K.J. Ray Liu, “A Complete Pipelined Parallel CORDIC Architecture for Motion Estimation," IEEE Transactions on Circuits and Systems II: Analog and Digital Signal Processing, Vol. 45, No. 6, June 1998, pp. 653-660.

7. E.F. Deprettere, A.A. J. de Lange, P. Dewilde, "The Synthesis and Implementation of Signal Processing Applications Specific VLSI CORDIC Arrays," Proceedings of the 1990 IEEE International Symposium on Circuits and Systems, New Orleans, LA, U.S.A., 1990, pp. 974-977.

8. M.D. Ercegovac, T Lang, "Ređundant and On-Line CORDIC: Application to Matrix Triangularization and SVD," IEEE Transactions on Computers, Vol. 39, No. 6, June 1990, pp. 725-740.

9. E. Grayver, B. Daneshrad, "Digital Frequency Synthesis Using a Modified CORDIC," Proceedings 1998 IEEE International Symposium on Circuit and Systems, Monterey California, May 31-June 3, 1998, pp. 241-244.

10. B. Haller, J Gotze, J.R. Cavallaro, "Efficient Implementation of Rotation Operations for High Performance QRD-RLS Filtering," Proceedings ASAP 97, Zurich Switzerland, July 14-16 1997, pp 162-174.

11. B. Haller, M. Streiff, U. Fleisch, R Zimmermann, "Hardware Implementation of a Systolic Antenna Array Signal Processor Based on CORDIC Arithmetic," 1997 IEEE International Conference on Acoustics, Speech, and Signal Processing, Munich Germany, April 21-24, 1997, pp. 4141-4144.

12. Grant Hampson, Andrew Paplinski, "Phase Shift Beamforming using CORDIC," International Symposium on Signal Processing and its Applications, Gold Coast Australia, August 1996, pp. 684-687.

13. Grant Hampson, Andrew Paplinski, "Hardware Implementation of an Ultrasonic Beamformer," Proceedings of the IEEE TENCON97 (Region 10 Annual Conference) Queensland University of Technology, Australia 1997, pp 227-230.

14. J.W. Jones, "Graphics Processor Using CORDIC Algorithm," IBM Technical Disclosure Bulletin, Vol. 22, No. 9, pp 4082-4085, February, 1980.

15. S. Kawasaki, M. Watabe, S Moringa, "A Floating Point VLSI Chip for the TRON Architecture," IEEE 
Micro, June 1989, pp. 26-44.

16. Jun Ma, Keshab Parhi, Ed F. Deprettere, "High-Speed CORDIC Based Parallel Weight Extraction for QRD-RLS Adaptive Filtering," Proceedings of the 1998 IEEE International Symposium on Circuits and Systems, Monterey California, May 31-June 3, pp. V-245-V-248.

17. T. Nakayama, H. Harigai, S. Kojima, H. Kaneko, H. Igarashi, T. Toba, Y. Yamagami, Y. Yano, “A 6.7MFLOPS Floating-Point Coprocessor with Vector/Matrix Instructions," IEEE Journal of Solid-State Circuits, Vol. 24, No. 5, October 1989, pp 1324-1330.

18. S.S. Nikolaidis, D.E. Metafas, C.E. Goutis, "CORDIC Based Pipeline Architecture for All-Pass Filters," 1993 IEEE International Symposium on Circuits and Systems, Chicago U.S.A. May 3-6, 1993, pp 19171920.

19. P. Rieder, C.V. Schimpfle, J.A. Nossek, "Realization jô '-fultiwavelet-Based Transform Kemels for Image Coding," IEEE International Symposium on Circuits and Systems Monterey California, May 31 June 3 1998, pp 538-541.

20. Roberto Sarmiento, Felix Tobajas, Valentin de Armas, Roberto Esper-Chain, J.F. Lopez, J.A. MontielNelson, A. Nunez, "A CORDIC Processor for FFT Computation and Its Implementation Using Gallium Arsenide Technology," IEEE Transactions on Very Large Scale Integration (VLSI) Systems, Vol. 6, No. 1, March 1998, pp. 18-30.

21. D. Timmermann, H. Hahn, B.J. Hosticka, G. Schmidt, "A Programmable CORDIC Chip for Digital Signal Processing Applications," IEEE Journal of Solid-State Circuits, Vol. 26, No. 9, September 1991, pp 1317-1321.

22. J. Vuori, J Skytta, "Implementation of a European Paging System Receiver Using CORDIC Algorithm," 8th European Signal Processing Conference Trieste Italy 1996, pp. 292-295.

23. J Vuori, "CORDIC Digital Phase-Locked Loop for FM Demodulation," Baltic Electronics Conference Tallinn Estonia, 1996, pp 105-108.

24. Erling H. Wold, Alvin M. Despain, "Pipeline and Parallel-Pipeline FFT Processors for VLSI Implementations," IEEE Transactions on Computers, Vol. C-33, No. 5, May 1984, pp. 414-426. 